\title{
Connecting two worlds
}

Citation for published version (APA):

Muschalik, C. (2020). Connecting two worlds: How conscious and unconscious cognitions shape health behaviors. [Doctoral Thesis, Maastricht University]. Maastricht University.

https://doi.org/10.26481/dis.20200207cm

Document status and date:

Published: 01/01/2020

DOI:

$10.26481 /$ dis. $20200207 \mathrm{~cm}$

Document Version:

Publisher's PDF, also known as Version of record

\section{Please check the document version of this publication:}

- A submitted manuscript is the version of the article upon submission and before peer-review. There can be important differences between the submitted version and the official published version of record.

People interested in the research are advised to contact the author for the final version of the publication, or visit the DOI to the publisher's website.

- The final author version and the galley proof are versions of the publication after peer review.

- The final published version features the final layout of the paper including the volume, issue and page numbers.

Link to publication

\footnotetext{
General rights rights.

- You may freely distribute the URL identifying the publication in the public portal. please follow below link for the End User Agreement:

www.umlib.nl/taverne-license

Take down policy

If you believe that this document breaches copyright please contact us at:

repository@maastrichtuniversity.nl

providing details and we will investigate your claim.
}

Copyright and moral rights for the publications made accessible in the public portal are retained by the authors and/or other copyright owners and it is a condition of accessing publications that users recognise and abide by the legal requirements associated with these

- Users may download and print one copy of any publication from the public portal for the purpose of private study or research.

- You may not further distribute the material or use it for any profit-making activity or commercial gain

If the publication is distributed under the terms of Article $25 \mathrm{fa}$ of the Dutch Copyright Act, indicated by the "Taverne" license above, 


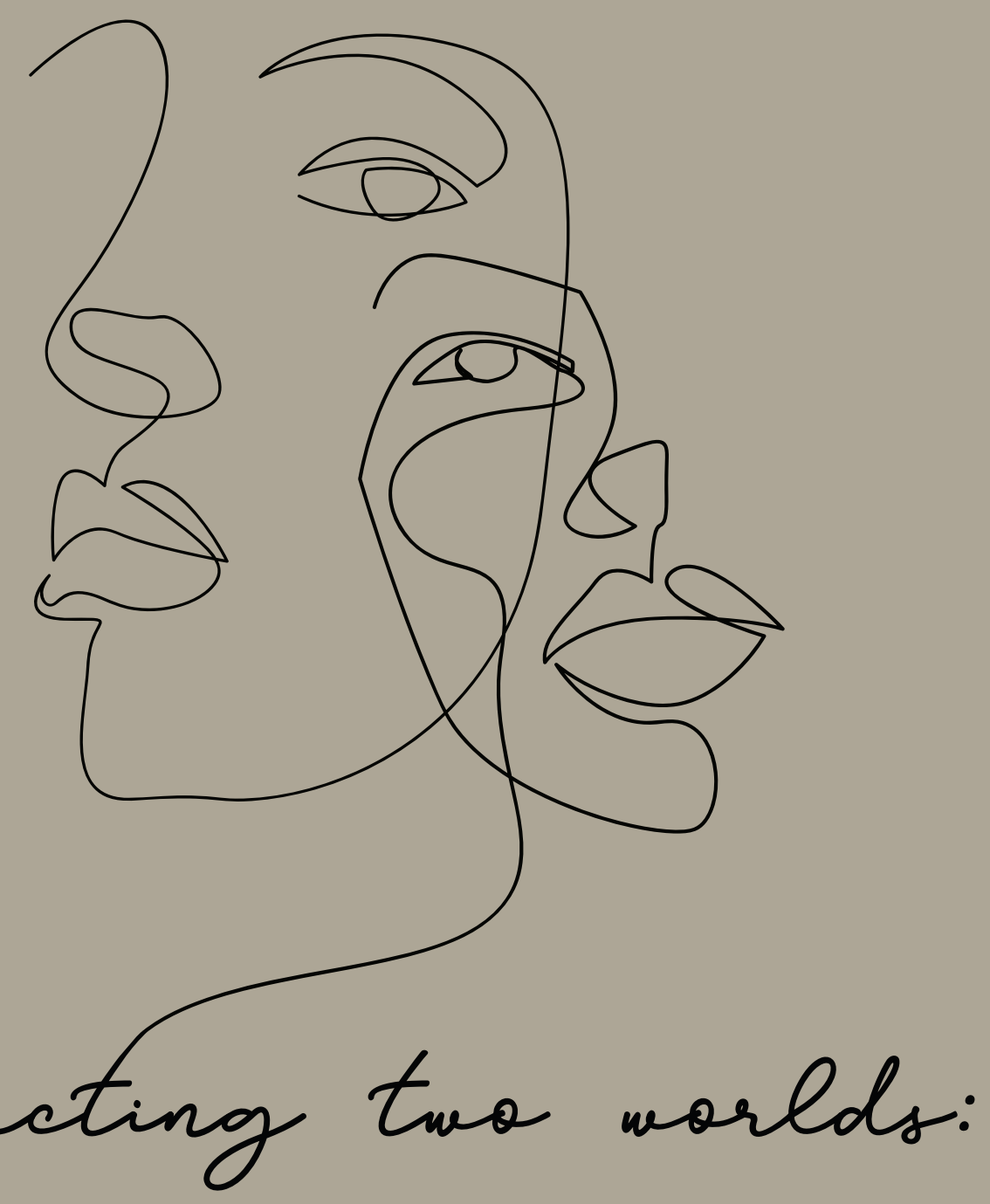

How conscious and unconscious cognition shape health behaviors

Carlin Muschalik 



\section{CONNECTING TWO WORLDS: \\ HOW CONSCIOUS AND UNCONSCIOUS COGNITIONS SHAPE HEALTH BEHAVIORS}

Carolin Muschalik 



\title{
CONNECTING TWO WORLDS: HOW CONSCIOUS AND UNCONSCIOUS COGNITIONS SHAPE HEALTH BEHAVIORS
}

\author{
DISSERTATION \\ To obtain the degree of Doctor at Maastricht University, \\ on the authority of the Rector Magnificus, Prof. dr. Rianne M. Letschert \\ in accordance with the decision of the Board of Deans, \\ to be defended in public \\ on Friday 7 February 2020, at 10:00 hours
}

by

\section{Carolin Muschalik}

born 1 May 1988 in Essen (Germany) 


\section{PROMOTERS}

Prof. dr. H. de Vries

Prof. dr. R. Crutzen

\section{COPROMOTOR}

Dr. I. Elfeddali, Tilburg University

\section{ASSESSMENT COMMITTEE}

Prof. dr. Stef Kremers (chair)

Prof. dr. Lilian Lechner, Open University

Prof. dr. Rob Ruiter

Prof. dr. Arjan Bos, Open University

Prof. dr. Trudy van der Weijden

Illustrations: Ikidogoooo, one line man, NikVector, Singleline, Neveshkin Nikolay, Pogorelova Olga/Shutterstock.com

Layout and printed by: Gildeprint - The Netherlands

ISBN: 978-94-640-2062-5

(C) Copyright 2020, Carolin Muschalik

All rights are reserved. No parts of this book may be reproduced, stored in a retrieval system or transmitted in any form or by any means, without the written permission from the author or, where appropriate, the publisher of the article. 
"Go then, go; your steps will not have been taken in vain" Domenico Laffi 



\section{TABLE OF CONTENTS}

CHAPTER ONE General Introduction 9

CHAPTER TWO A longitudinal study on how implicit attitudes and explicit 19 cognitions synergistically influence physical activity intention and behavior

CHAPTER THREE Implicit attitudes and explicit cognitions jointly predict reduced red meat intake: a three-wave longitudinal study

CHAPTER FOUR Mindfulness is not associated with dissonant attitudes but enhances the ability to cope with them

CHAPTER FIVE Does the discrepancy between implicit and explicit attitudes moderate the relationships between explicit attitude and (intention to) being physically active?

CHAPTER SIX Pathways of implicit attitudes, explicit attitudes, and behavior 
Chapter 
ene:

General Introduction 


\section{THEORIES TO UNDERSTAND BEHAVIOR}

In order to develop successful (health) behavior change interventions, it is crucial to first understand what determines these behaviors. Once knowledge about relevant determinants is established, intervention developers are able to address these specific determinants, thereby making it more likely that the desired change is achieved (Bartholomew Eldredge et al., 2016). In the last decades, multiple theories were developed and tested empirically in order to provide a solid ground for the development of interventions. Most of these theories were based on the explicit cognition approach. This approach focuses on conscious determinants that have been shown to predict behavior to a large extent (Fishbein \& Ajzen, 2011; Godin, Bélanger-Gravel, Eccles, \& Grimshaw, 2008; Hagger, Chatzisarantis, \& Biddle, 2002). The implicit cognition approach, which evolved in the last two decades, does not only take conscious but also rather unconscious and automatic determinants of behavior into account (Gawronski \& Bodenhausen, 2006; Strack \& Deutsch, 2004). In order to obtain a more extensive understanding of health behaviors, different theories should be integrated with each other (De Vries, 2017; Peters \& Crutzen, 2017). Hence, as behavior is assumed to be neither influenced solely by conscious nor solely by unconscious mechanisms, a challenge is to investigate whether integral approaches, in which both mechanisms are integrated, are more helpful for understanding behavior formation. Therefore, both approaches will be addressed in this dissertation with the aim to integrate them - including the development and testing of new hypotheses (De Vries, 2017) - rather than to examine the two approaches in isolation from each other, which has long been the case (Haddock \& Maio, 2004). This was mainly a result of the long believed, but by now outdated assumption that behavior is either an outcome of intentional processes or of automatic processes. In the following section, the two approaches and their underlying assumptions and processes are explained and gaps in the current literature are addressed.

\section{THE EXPLICIT COGNITIONSAPPROACH}

Traditional research on explaining and predicting health behaviors has mainly been focused on theories that emphasize a reasoned and deliberate approach towards behavior formation. These models focus on explicit cognitions, which individuals can elaborate on, are consciously accessible and hence, can be measured by means of questionnaires or interviews. The I-change model (De Vries, 2017; De Vries, Mesters, Van de Steeg, \& Honing, 2005) integrates several social-cognitive models and will be described in order to depict the most influential explicit determinants for health behaviors. According to the motivational phase of the model, an individual's intention is one of the most proximal determinants for behavior. Intention in turn is shaped by the following three key determinants. Firstly, by an individual's explicit attitude towards the behavior (comprised of perceived pros and perceived cons, i.e. the perceived 
advantages and disadvantages of the behavior). Secondly, by social influence (comprised of social modeling and social norms, i.e. how people in the individual's environment behave and the perceived social pressure to perform a behavior) and thirdly, by self-efficacy (i.e. the perceived ability to perform the respective behavior). Besides the motivational phase, the I-change model also distinguishes between a pre- and a post-motivational phase, each with relevant determinants, such as knowledge, risk perception, or cues to action. The focus of this dissertation will, however, be on the abovementioned motivational phase, which shows similarities regarding relevant determinants with the Reasoned Action Approach, one of the most used models in health psychology (Fishbein \& Ajzen, 2011).

Many studies tested and found support for the above-mentioned relationships between explicit attitude, social norms, self-efficacy, intention, and behavior. Especially the latter relationship, the translation of intention into actual behavior, has been investigated in multiple studies (Albarracin, Johnson, Fishbein, \& Muellerleile, 2001; Bagozzi \& Warshaw, 1990; Norman, Conner, \& Bell, 1999; Sheeran \& Orbell, 2000). Whereas the relationship between intention and behavior has been shown to be relatively strong in cross-sectional studies, the same relationship has repeatedly been shown to be rather weak in longitudinal studies. More precisely, intention was found to explain between $19 \%$ and $38 \%$ of variance in actual behavior (Armitage \& Conner, 2001; Sheeran, 2002; Sutton, 1998), thereby leaving a great proportion of behavior unexplained. This issue, labeled as the 'intention-behavior gap' (e.g. an individual reports a high intention to perform a behavior but fails to act accordingly), has been observed across various health behaviors, such as physical activity, alcohol consumption, condom use, and smoking behavior (Sheeran, 2002; Webb \& Sheeran, 2006). Also, intention cannot be fully explained by the determinants explicit attitude, social norms, and self-efficacy. According to a meta-analytic review, around $40 \%$ of the variance in intention is explained by these three determinants (Armitage \& Conner, 2001). In order to understand both intention and behavior formation even better, it appears therefore necessary to also take those factors into account that are activated automatically and might even occur outside an individual's conscious awareness.

\section{THE IMPLICIT COGNITIONSAPPROACH}

According to the implicit approach, behavior is not solely determined by explicit, conscious processes, but also by implicit, rather unconscious processes. Contrary to explicit cognitions, implicit cognitions are fast, activated automatically, and can occur without an individual's conscious awareness (Fazio \& Olson, 2003). Implicit cognitions are, for example, implicit attitudes, attentional biases, or automatic approach or avoidance tendencies. Implicit attitudes are understood as positive or negative associations with an object or behavior, which occur automatically. Therefore, individuals are not necessarily aware of their implicit attitudes and do not have conscious access to them most of the time (Rydell \& McConnell, 2006). When 
implicit attitudes towards a behavior are positive, the individual is more likely to engage in the respective behavior. An attentional bias is understood as the tendency to pay attention to certain stimuli in one's environment while at the same time ignoring other stimuli. For example, a smoker might detect more easily stimuli related to smoking (e.g. ash trays, cigarettes) in his or her environment, which appear appealing to him or her due to the repeated use of them and the associated positive experiences. As a consequence, the individual is automatically guided towards the stimuli and is more likely to perform the respective behavior associated with those stimuli (i.e. smoking) (Begh et al., 2013; Mogg, Bradley, Field, \& De Houwer, 2003). Approach and avoidance tendencies are understood as automatic propensities to either move towards or away from certain stimuli. Hence, a strong automatic approach tendency towards stimuli belonging to a behavior (e.g. cigarettes) makes an individual more likely to perform the behavior related to the stimuli (e.g. smoking) (Palfai \& Ostafin, 2003; Veenstra \& de Jong, 2010). The opposite is true for automatic avoidance tendencies (Wiers, Eberl, Rinck, Becker, \& Lindenmeyer, 2011). As people are not necessarily aware of their implicit cognitions, they cannot be assessed by means of questionnaires or interviews. So, instead computerized reaction time tasks are used to measure implicit cognitions (e.g. Gawronski \& De Houwer, 2014; Karpinski \& Steinman, 2006). For example, to assess implicit attitudes, participants are asked to sort positive and negative words/pictures as well as representative words/pictures for the target (e.g. smoking) as quickly as possible to two given categories, which are associationcongruent (e.g. smoking and negative) or association-incongruent (e.g. smoking and positive) (e.g. Greenwald, McGhee, \& Schwartz, 1998; Karpinski \& Steinman, 2006). In order to assess attentional biases, the dot probe task is most commonly used (MacLeod, Mathews, \& Tata, 1986). In this task, subjects are presented with two stimuli (words or pictures) representing the target or not, next to each other on a computer screen. When the two stimuli disappear, a dot probe appears in place of one of the stimuli, whose location participants are asked to indicate as quickly and accurately as possible. Faster reaction times to the dot probe indicate a biased attention towards the stimuli seen earlier on the same position. To assess approach/avoidance tendencies, participants are, for instance, instructed to respond to pictures, representing the target (e.g. cigarettes to represent smoking) or neutral objects by pushing them away or pulling them towards themselves regardless of the content but depending on the format of the picture (e.g. landscape or portrait) (e.g. Rinck \& Becker, 2007). It has been shown that although the picture content should be irrelevant, a stimulus-response compatibility effect is present. This means that smokers are usually faster to pull smoking related pictures towards them than to push them away, indicating an approach tendency towards smoking related stimuli. Based on the performance of these reaction time paradigms, inferences of an individual's implicit cognitions can be drawn. 


\section{DUAL-PROCESS MODELS}

The assumption that behavior is predicted by both, explicit and implicit cognitions, is depicted in dual-process models. According to these models, a reflective system - comprised of reasoned, conscious, and deliberate cognitions, hence explicit cognitions - and an impulsive system - comprised of fast and automatic tendencies, hence implicit cognitions - influence behavior (Gawronski \& Bodenhausen, 2006; Strack \& Deutsch, 2004). According to Perugini (2005), there exist three different ways in which explicit and implicit cognitions jointly predict behavior. First, according to the additive pattern, both types of cognitions, explicit and implicit cognitions, explain unique variance in terms of behavior. Second, according to the double dissociation pattern, explicit cognitions predict deliberate behavior best and implicit cognitions predict spontaneous behavior best. Lastly, according to the interactive pattern, both types of cognitions interact in the prediction of behavior, i.e. one type of cognition weakens or reinforces the effect of the other type of cognition on behavior. Although these patterns have been tested separately in a couple of studies (Conroy, Hyde, Doerksen, \& Ribeiro, 2010; Perugini, 2005), these studies did not test these three patterns within one study. Therefore, it remains unclear, whether there exists a common pattern across different health behaviors and whether two (or more) patterns can co-occur, i.e. can behavior be predicted in accordance with an additive as well as in accordance with an interactive pattern.

Furthermore, although dual-process models speak of explicit and implicit cognitions in general, these are mainly interpreted and investigated as explicit attitudes and implicit attitudes. Several studies looked at the effect of explicit and implicit attitudes on health behaviors and found both types of attitudes to be related to behavior (McConnell \& Leibold, 2001). As depicted in the aforementioned social-cognitive models, however, a large part of behavior is not solely determined by the explicit cognition attitude, but also by self-efficacy, social norms, and intention. In order to understand health behavior even better, one possibility would be the theoretical integration of implicit cognitions with explicit cognitions and to investigate the specific mechanism of influence on behavior. Only then a fuller understanding of behavior formation might be possible. This in turn may contribute to the development of more successful health interventions that are aiming to improve people's health and wellbeing. Additionally, most social-cognitive models postulate that intention is one of the most proximate determinants for behavior. Thereby intention builds an important prerequisite for behavior. As the explicit cognitions explicit attitude, social influence, and self-efficacy only explain around $40 \%$ of variance in intention (Armitage \& Conner, 2001), the question arises whether implicit cognitions also play a role in intention formation, and if so, which role. Understanding the process of intention formation even better could offer a first promising step in the direction of improving behavior change interventions.

Chapter 2 and Chapter 3. As a result of the aforementioned shortcomings, we report two studies in the second and third chapter of this dissertation, in which we investigated whether explicit cognitions and implicit attitudes predict the intention to perform a behavior 
separately from each other (additive pattern) or whether they interact in the prediction of intention to perform a behavior (interactive pattern). The same questions were addressed with behavior as outcome - focusing on two different behaviors (i.e., physical activity and red meat consumption) that are relevant to public health.

\section{M PLICIT-EXPLICIT DISCREPANCY}

According to the above-mentioned dual-process models, implicit attitudes and explicit attitudes are evaluative, hence they are negatively or positively laden. This means that implicit attitudes and explicit attitudes towards the same object or behavior can be coherent (e.g. both types of attitude are positive or both types are negative), but that they can also be dissonant. For example, the implicit attitude towards a behavior can be positive, whereas the explicit attitude towards the same behavior is negative, or vice versa. The occurrence of this phenomenon, named implicit-explicit discrepancy (IED), has been demonstrated in several studies (e.g. Brinol, Petty, \& Wheeler, 2006; Petty, Tormala, Brinol, \& Jarvis, 2006; Rydell \& McConnell, 2006; Swanson, Swanson, \& Greenwald, 2001). For instance, smokers were found to have negative implicit attitudes and positive explicit attitudes towards smoking at the same time (Swanson et al., 2001).

More importantly, IED has been shown to be related to behavior. Findings regarding this relationship are mixed. For example, in a study on information processing, people with stronger IED regarding certain self-dimensions, engaged in more information processing (Brinol et al., 2006). In a study on smoking, smokers with higher IED were more likely to light a cigarette than people with lower IED (Maliszewski, 2011) and in a study regarding chocolate consumption, people with higher IED showed more disinhibited chocolate consumption than people with lower IED (Goldstein et al., 2014). These findings demonstrate that implicit and explicit attitudes can be in conflict and that this conflict in turn impacts behavior. This can be explained by means of Festinger's cognitive dissonance theory (1957). According to this theory, people are striving for consistency between their own attitudes, beliefs, and behavior at any time (Dalege, Borsboom, van Harreveld, \& van der Maas, 2018). In case dissonance occurs, people are motivated to resolve it as it is assumed to be accompanied by negative feelings. To do so, there exist different ways, such as ignoring or denying the information that conflict with existing cognitions, justifying one's behavior or cognition, adding new cognitions, or by changing one's behavior (Festinger, 1957). As an imbalance between implicit and explicit attitudes causes dissonance, people are motivated to resolve the dissonance and accompanied negative feelings (Rydell, McConnell, \& Mackie, 2008). It is plausible that in the aforementioned studies, participants applied the latter strategy of behavior change to resolve IED. This is also regarded as the most preferred strategy to resolve dissonance (Festinger, 1957), which was also the conclusion from a literature review (Stone \& Fernandez, 2008). As IED can have negative effects on health behaviors, the question arises whether possible third 
factors exist that (a) are able to reduce dissonance between implicit and explicit attitudes (b) are able to reduce the effect of dissonance on behavior. These questions will be further elaborated on in the following section and are addressed in depth in Chapter 4.

\section{FACTORS ASSOCIATED WITH IMPLICIT-EXPLICIT D I S C REPA NCY}

Until now, a few factors have been discussed to be related to the level of congruence/ dissonance between attitudes. Besides methodological factors, such as a high correspondence of the content between the explicit and implicit measure, (Hofmann, Gschwendner, Nosek, \& Schmitt, 2005), individual factors have been discussed as well.

One factor is the importance of an attitude (Hofmann, Gschwendner, Nosek, et al., 2005), which relates to the significance that an individual attaches to the attitude (Boninger, Krosnick, \& Berent, 1995). When an attitude is important, an individual is more likely to seek out relevant information and to think about the attitude more frequently (Bizer \& Krosnick, 2001). As a consequence and contrary to unimportant attitudes, important attitudes are more stable and accessible as well as more likely to be activated automatically when the attitude object is encountered (Roese \& Olson, 1994). Implicit measures capture automatic evaluations of an object. Hence, when certain automatic evaluations are highly accessible, due to their importance, they have been shown to be more congruent with the evaluations that are assessed by means of explicit measures (Karpinski, Steinman, \& Hilton, 2005; Nosek, 2005). Another factor discussed in this context is preference for consistency (Thrash, Elliot, \& Schultheiss, 2007), which is understood as an individual's motivation to seek congruence between his or her cognitions. Although it is assumed that individuals in general are motivated to seek congruence (Festinger, 1957), the strength of this motivation may vary. Indeed people with a stronger distinct preference for consistency showed less IED compared to people with a less distinct preference for consistency (Thrash et al., 2007). Moreover, introspective access has been discussed and investigated as a possible moderator of IED (Hofmann, Gschwendner, Nosek, et al., 2005; Hyde, Doerksen, Ribeiro, \& Conroy, 2010; Thrash et al., 2007). Studies regarding introspective access and IED yielded mixed results. In the study of Thrash et al. (2007), introspective access, which is defined as a person's sensitivity to bodily states and tendency to focus on thoughts and feelings, moderated IED. This was, however, not the case in the study of Hyde et al. (2010). As a result of these mixed findings, a similar but broader concept has been proposed to foster congruency between attitudes, namely mindfulness. Mindfulness is defined as "the awareness that emerges through paying attention on purpose, in the present moment, and nonjudgmentally to the unfolding of experience moment by moment" (Kabat-Zinn, 2003, p. 145) and can be regarded as a trait-like characteristic. However, people can also foster this skill by practicing certain techniques (Hölzel et al., 2011; Shapiro, Oman, Thoresen, Plante, \& Flinders, 2008). Contrary to less mindful people, more mindful 
individuals are more aware and attentive to inner (physical and psychological) processes and events, hence also to implicit cognitions, which are normally less accessible (Brown \& Ryan, 2003; Koole, Govorun, Cheng, \& Gallucci, 2009). This insight in turn is assumed to enable the person to translate his or her implicit cognitions more accurately to an explicit report, with greater congruence between implicit and explicit attitudes as result. Until now, mindfulness as possible moderator of IED has only been investigated in the context of self-related constructs, such as self-esteem (Koole et al., 2009), but not regarding attitudes. Investigating whether mindfulness also moderates congruency between implicit and explicit attitudes may help in the endeavor to diminish the possible negative effect that dissonant attitudes can have on health behaviors, especially since mindfulness is a skill that can be trained in any individual. Chapter 4. In the fourth chapter, we investigated, whether mindfulness is associated with IED and whether mindfulness moderates the relationships between IED and behavior and IED and intention.

IED has not only been shown to be associated with behavior, but also to moderate the relationship between explicit attitude and behavior (Karpen, Jia, \& Rydell, 2012). More precisely, explicit attitudes were not a significant predictor for alcohol consumption anymore when IED was strong but a significant predictor when IED was low. This is important since the ability of explicit attitudes to predict behavior (Ajzen \& Fishbein, 1977; Kraus, 1995) has made them a key construct in many behavioral theories (De Vries, 2017; Fishbein \& Ajzen, 2011) and a relevant determinant across a wide range of health behaviors. As a result, it is recommended to change explicit attitudes in order to change behavior (Chatzisarantis, Hagger, Biddle, \& Smith, 2005) and many health interventions do indeed have this focus (e.g. Bingham et al., 2011; Juraskova et al., 2012; Maynard et al., 2015; Newton, Teesson, Vogl, \& Andrews, 2010; Olson, Gaffney, Starr, \& Dietrich, 2007). If, however, explicit attitudes lose their predictive power for behavior in the presence of IED, it becomes questionable whether health interventions with this very focus can still be successful or whether they should take the congruency of implicit and explicit attitudes into account. Moreover, explicit attitudes are strongly associated with intention (De Vries, 2017; Fishbein \& Ajzen, 2011). That is, the effect of explicit attitude on intention is twice as strong as the effect of social norms and also stronger than the effect of self-efficacy on intention (Hagger et al., 2002). Concomitant with that, explicit attitudes have been shown to explain around $30 \%$ of variance in intention. Therefore, and due to the importance of intention in the process of behavior change, the question is whether IED does not only moderate the relationship between explicit attitudes and behavior but also between explicit attitude and intention.

Chapter 5. The fifth chapter provides more insight into the question whether IED moderates the relationship between explicit attitude and behavior as well as between explicit attitude and intention. 


\section{PATHWAYS OF IMPLICIT ATTITUDES, EXPLICIT ATTITUDES, AND BEHAVIOR}

Explicit attitudes are well-known to be related to a person's behavior (Greenwald, Poehlman, Uhlmann, \& Banaji, 2009; McEachan et al., 2016) and a person's behavior in turn is known to feed back to a person's explicit attitude. For example, when a person realizes that he/she performed a behavior that contradicts his/her former explicit attitude, the former attitude will be adjusted in accordance with the aforementioned cognitive dissonance theory (Festinger, 1957). Also biased scanning can contribute to explain this phenomenon: an individual conducts a biased search of existing beliefs that confirm the legitimacy of the behavior that was performed (Albarracín \& Wyer Jr, 2000; Janis \& King, 1954). Although it is well-known that there exists an interplay between explicit attitudes, behavior, and explicit attitudes in turn, it has not been investigated yet whether this reciprocal relationship also applies to implicit attitudes and behavior. Hence, it is unclear whether implicit attitudes are shaped by a person's behavior, or in contrast act only as a precursor for behavior, or both. Also dual-process models remain silent about this possible interaction. As previous research has suggested that implicit attitudes might be sensitive to the recent experience of the behavior (Gawronski \& Bodenhausen, 2006; Rudman, 2004), we investigated whether behavior and implicit attitudes influence each other over time by using full cross-lagged models.

Moreover, it has not been investigated yet whether implicit and explicit attitude influence each other over time. Former studies demonstrated that implicit attitudes can change naturally over time (Gawronski, Morrison, Phills, \& Galdi, 2017; Hyde, Elavsky, Doerksen, \& Conroy, 2012) and it has been proposed that these changes could possibly transition into explicit measures, when the new (implicit) association is regarded as valid (Gawronski et al., 2017). Furthermore, it has been suggested that this interplay can work in the other direction as well. More specifically, new information concerning a behavior is assumed to be reflected in an individual's explicit attitude. This in turn might lead to a new (implicit) association between an object or behavior and its evaluation (Gawronski et al., 2017). Shedding light on these relationships might aid in a better understanding of whether changing one type of attitude leads to a change in the other type of attitude or not. If this is the case, interventions could focus on changing one type of attitude only, making it thereby redundant to modify both types of attitudes in order to change behavior.

Chapter 6. In Chapter 6, we address not only the question whether the performance of a behavior feeds back to a person's implicit attitude, but also whether changes in implicit or explicit attitudes lead to changes in explicit and implicit attitudes over time, respectively.

Chapter 7. In the final chapter of this dissertation, the findings described in the previous chapters are summarized, reflected on, and discussed. Moreover, implications of the conducted studies are addressed and suggestions for future research are proposed. 
Chapter 


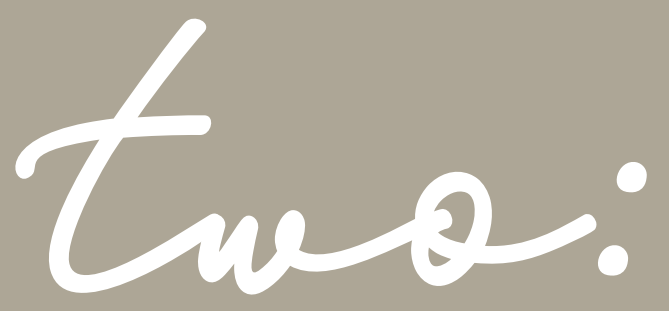

A longitudinal study on how implicit attitudes and explicit cognitions synergistically influence physical activity intention and behavior

Muschalik, C., Elfeddali, I., Candel, M. J. J. M., \& de Vries, H. (2018). BMC psychology, 6(1), 18. 


\section{A B S T R A C T}

Background: Strategies to promote physical activity (PA) focus mainly on changing or fostering explicit cognitions and are only modestly effective. Contemporary studies suggest that, as well as explicit cognitions, implicit cognitions influence health behavior, such as PA, and that implicit processes interact with the intention to be active. Relatively little is known about whether implicit processes interact with other explicit cognitions which determine PA intention and behavior, i.e. self-efficacy. The aim of the current study was to investigate the direct effects of explicit cognitions and implicit attitudes on PA behavior as well as interactions between them regarding intention and behavior prediction.

Methods: In a longitudinal study, participants $(\mathrm{N}=340)$ completed self-report measures of explicit cognitions (perceived pros, perceived cons, social norms, social modeling, self-efficacy, intention) and activity levels, as well as a SingleCategory Implicit Association Task to measure implicit attitudes towards PA at baseline (T0), and at one (T1) and 3 months thereafter (T2).

Results: Hierarchical multiple regressions revealed that T0-positive implicit attitudes moderated the relationship between T0 self-efficacy and T1 PA. Similarly, T0neutral implicit attitudes were associated with the relationship between T0 intention and T1 PA. Negative implicit attitudes strengthened the negative relationship between perceived cons and intention at baseline; neutral or positive implicit attitudes strengthened the positive relationship between self-efficacy and intention. At the follow-ups, the relationship between social modeling and intention was strengthened by negative implicit attitudes.

Conclusion: This study revealed important insights into how implicit attitudes and explicit cognitions synergistically predict PA intention and behavior. As well as targeting explicit cognitions, steering a person's implicit attitude towards a more positive one, i.e. by implicit cognitive trainings, could help to increase both PA intention and behavior. 


\section{B ACKGROUND}

Insufficient physical activity is known to cause non-communicable diseases such as hypertension, obesity, cancer, type 2 diabetes, and cardiovascular diseases (U.S. Department of Health and Human Services, 2008; Warburton, Charlesworth, Ivey, Nettlefold, \& Bredin, 2010; World Health Organization [WHO], 2010). Consequently, the need to promote physical activity (PA) has become an important public health goal (Samuelson, 2004). Yet, the recommended level for PA - i.e. to be at least moderately physically active for 150 minutes per week (WHO, 2003) - is still not met by $31 \%$ of the world's population (Hallal et al., 2012). To help develop more effective interventions, it is necessary to gain deeper insight into the determinants that predict PA. There are two paradigms that can be applied to explain health behaviors. The first focuses on identifying explicit beliefs of people concerning a behavior, and is inspired by a set of complementary social cognitive and ecological models which summarize multiple levels of influences on behavior (Ajzen, 1991; Albert, 1986; Conner \& Norman, 2005). Explicit beliefs are determinants which people are aware of, can express consciously, and are measured by self-reported questionnaires. For instance, the explicit attitude towards a behavior (e.g. 'Being physically active is very good for my health') or a person's reported ability to engage in a behavior when being confronted with challenging situations, called self-efficacy (e.g. 'I find it hard to be sufficiently physically active when I am stressed' or 'I find it hard to be sufficiently physically active when I dislike the activity'). The second paradigm focuses on unconscious processes which persons may not be aware of but which still influence their behavior, called implicit processes (Sheeran, Gollwitzer, \& Bargh, 2013; Strack \& Deutsch, 2004). Implicit attitudes are one type of implicit process. They are automatically occurring attitudes of which people are less aware and to which people do not initially have conscious access (Rydell \& McConnell, 2006). To assess implicit attitudes, computerized reaction time tasks are used, i.e. the Implicit Association Test (IAT) (Greenwald et al., 1998). While several studies have applied both the explicit and the implicit paradigms, only a few focus on how to combine these approaches. The present study attempts to integrate them.

An example of the explicit paradigm is reflected by the l-change model (De Vries et al., 2005) which has also been used to assess and change PA-related cognitions and behaviors (De Vries, Kremers, Smeets, \& Reubsaet, 2008; Schulz et al., 2014; Smeets, Kremers, Brug, \& De Vries, 2007). According to the I-change model - which integrates aspects from socialcognitive models, i.e. the Theory of Planned Behavior (Ajzen, 1991), the Trans Theoretical Model (Prochaska \& Velicer, 1997), Social Cognitive Theory (Albert, 1986) and Goalsetting Theory (Locke \& Latham, 1990) - intention is one of the most proximal conscious determinants for behavior. Intention in turn is determined by the attitude to the behavior (comprised of perceived pros and perceived cons regarding a behavior, e.g. 'When I am sufficiently active I have more energy' or 'Being sufficiently active costs me a lot of effort'), social influence (the perception of the norms and behavior of people in the social environment as well as the perceived social support, e.g. 'Most of my friends think that I need to be sufficiently active' or 
'Most of my friends are sufficiently active') and self-efficacy (whether a person perceives him or herself as capable of performing a behavior when confronted with obstacles). Individuals with high levels of self-efficacy are more likely to exert effort to perform a behavior and are therefore more likely to succeed, whereas people with low levels are more likely to fail (Stajkovic \& Luthans, 1998). PA behavior indeed has a reliable correlation with intention, and intention in turn acts as a mediator between the explicit cognitions such as attitude, knowledge, selfefficacy, social norms and behavior and self-efficacy also has a direct effect on PA behavior (French et al., 2005; Hagger et al., 2002; Park \& Kim, 2008; Sallis et al., 1986; Terry, Erickson, \& Johnson, 1977). In most of the publications on PA, this paradigm is the most dominant one and most interventions aim to increase PA levels by changing explicit cognitions. A review concludes, however, that this approach is only modestly effective (Conn, Hafdahl, \& Mehr, 2011), and the contemporary idea is that implicit cognitions need to be taken into account, in addition to explicit cognitions.

The relatively new concept of combining implicit and explicit cognitions is reflected in dual process models (Hofmann, Friese, \& Wiers, 2008; Sheeran et al., 2013; Strack \& Deutsch, 2004; Wilson, Lindsey, \& Schooler, 2000). According to the Reflexive-Impulsive Model (RIM) (Strack $\&$ Deutsch, 2004), an example of a dual-process model, an impulsive and a reflective system exist, both of which guide behavior. Whereas the reflective system is composed of reasoned, deliberate, and conscious motives, the impulsive system is a composition of affective responses and automatically associated behavioral tendencies. According to the RIM, the reflexive and impulsive systems can influence behavior in different ways. One way is the double dissociation pattern (Perugini, 2005), according to which spontaneous behavior is predicted best by the impulsive system, and deliberate behavior by the reflexive system (Dovidio, Kawakami, \& Gaertner, 2002; Hofmann, Rauch, \& Gawronski, 2007; McConnell \& Leibold, 2001; Neumann, Hülsenbeck, \& Seibt, 2004). Another potential way of how the two types operate is referred to as the additive pattern (Perugini, 2005), meaning that both systems explain unique variance in one behavior. This pattern has indeed been shown for purchasing healthy food (Prestwich, Hurling, \& Baker, 2011), dental flossing (Millar, 2011) and also with regard to PA. Concerning the latter behavior, it has been demonstrated that automatic, less conscious processes play a unique role alongside explicit cognitions in explaining past (Bluemke, Brand, Schweizer, \& Kahlert, 2010; Calitri, Lowe, Eves, \& Bennett, 2009) and future PA behavior (Conroy et al., 2010) as well as the maintenance of PA (Chevance, Héraud, Varray, \& Boiché, 2017; Endrighi et al., 2016). From this perspective, it follows that PA is regulated by both impulsive (or implicit) and reflective (or explicit) cognitions. This conclusion was indeed reached in a recent review (Rebar et al., 2016).

Although explicit and implicit constructs have been shown to play a role in determining PA, it is not clear which of the above-stated patterns can be applied to PA. Conroy and colleagues (2010) showed that implicit and explicit cognitions explain unique variance in PA behavior, i.e. favoring the additive pattern. Berry and colleagues (2011), however, challenged this approach and concluded from their study that implicit and explicit cognitions are not only directly 
related to PA behavior, but that implicit attitudes interact with the intention to be active. This is in line with a third way of operating, namely the interactive pattern, meaning that the reflective and impulsive systems interact synergistically to predict behavior (Perugini, 2005). Also, Cheval and colleagues (2015) found that impulsive processes interacted with PA intentions. More precisely, PA intentions predicted PA behavior when the impulsive approach tendencies toward the opposite behavior of PA, namely sedentary behavior, were low or moderate. By contrast, strong impulsive approach tendencies toward sedentary behavior blocked the effect of intention on behavior. These findings suggest that the way implicit and explicit processes jointly influence PA might be more complex than so far assumed.

Although different patterns of influence have been demonstrated, we argue that the two patterns are not necessarily mutually exclusive. Implicit attitudes and explicit determinants could both have a direct effect on behavior (additive pattern) and also interact with each other (interactive pattern). Until now, the two operating models have not been tested in a single study. Furthermore, former studies, such as the one by Cheval and colleagues (2015), investigated the interactive pattern only between impulsive tendencies and the explicit construct intention. We aim to take this research approach one step further and raise the question whether implicit processes might also interact with the above-mentioned explicit cognitions that predict intention (perceived pros, perceived cons, social norms, social modeling, self-efficacy) and intention itself. Just as impulsive tendencies in the study by Cheval et al. (2015) either reinforced or disinhibited the relationship between intention and behavior, we assume that implicit attitudes could have a reinforcing or inhibiting effect on the relationship between explicit cognitions and intention. For instance, it is conceivable that a person who perceives many pros regarding PA has an even stronger intention to become active when he or she unconsciously evaluates the behavior as positive. If, however, the same person evaluates PA unconsciously as negative, we expect this negative implicit attitude to inhibit the effect of perceived pros on intention. The similar pattern of reasoning could be applied to the other predictors of intention. Although intention does not necessarily lead to behavior, it still accounts for $23 \%$ of the variance in PA (McEachan, Conner, Taylor, \& Lawton, 2011) and is regarded as an important step in the adoption and maintenance of behavior and as a good predictor in the context of protective behaviors such as PA (Sheeran, Klein, \& Rothman, 2017). Shedding light on the joint role that implicit attitudes and explicit cognitions play in intention formation could help to further elucidate this process.

The aim of the present study was three-fold. First, we investigated the direct effects of implicit attitudes and explicit cognitions on PA behavior (figure 2.1). As found in the former two studies (Cheval et al., 2015; Conroy et al., 2010), we expect both implicit attitudes and explicit cognitions to predict unique variance in PA behavior $(\mathrm{H} 1)$. Second, interactions between implicit attitudes and intention and implicit attitudes and self-efficacy were examined (figure 2.2). Just like Cheval and colleagues (2015) and in line with an interactive pattern of behavior prediction, we assume implicit attitudes also moderate the relationship between intention and PA and self-efficacy and PA (H2). Third, interactions between explicit cognitions and implicit attitudes 
were assessed (figure 2.3). We expect that the positive influence of the explicit cognitions (perceived pros, social norms, social modeling and self-efficacy) on intention is strengthened by positive implicit attitudes. The negative effect of perceived cons on intention is expected to be weakened by positive implicit attitudes but strengthened by negative implicit attitudes (H3).

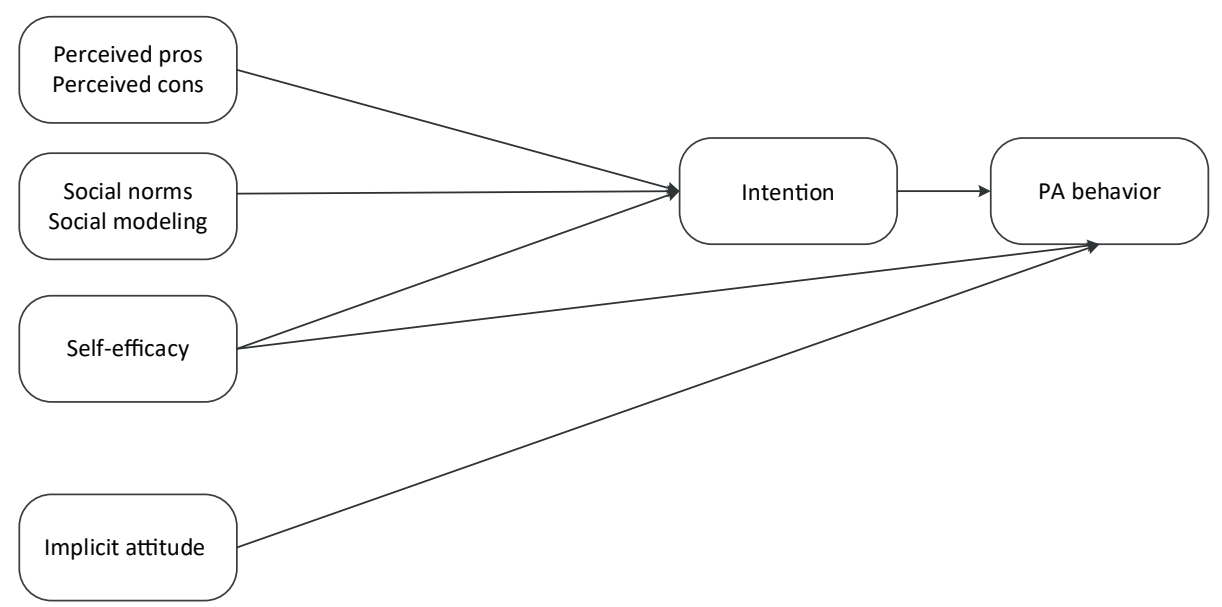

Figure 2.1 | Assessing the direct effects of the explicit cognitions (perceived pros, perceived cons, social norms, social modeling, self-efficacy, intention) and the implicit attitude on PA behavior

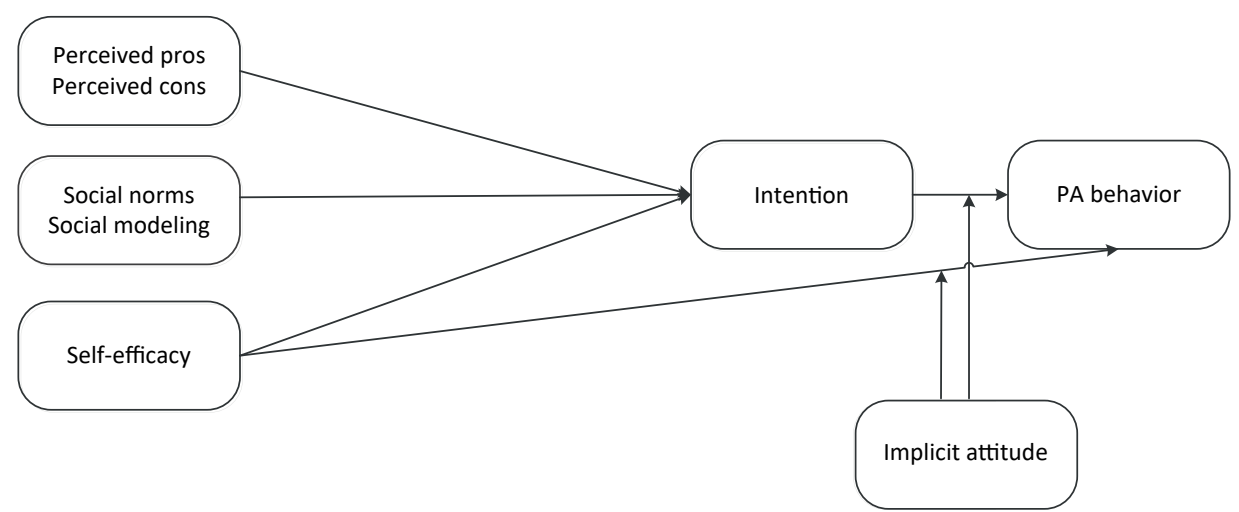

Figure 2.2 | Assessing the interaction effects of implicit attitudes on the relation between self-efficacy and PA behavior and the relation between intention and PA behavior 


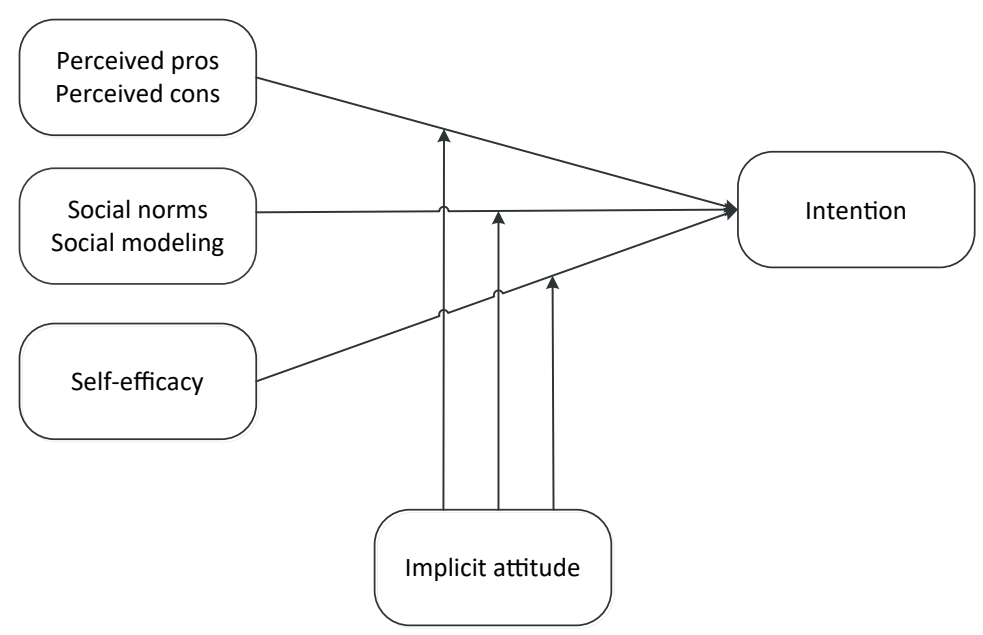

Figure 2.3 Assessing the interaction effects of implicit attitudes on the relations between perceived pros and ntention, perceived cons and intention, social norms and intention, social modeling and intention, and self-efficacy and intention

\section{METHODS}

\section{Design}

A longitudinal study was conducted with a baseline measurement (T0), a follow-up after one month (T1) and another follow-up after three months (T2).

\section{Power analysis}

With the assumption of a small effect size $(f 2=0.023$ ) for a main effect or interaction effect of implicit attitude and a test power set at 0.80 with a type I error rate of $\alpha=0.05$ for two-sided testing, power analysis revealed that 330 respondents are needed. Anticipating a drop-out rate of $20 \%$, we aimed to conduct the first session of the study with 413 participants in order to have data from at least 330 participants at the first follow-up.

\section{Participants and recruitment}

Following approval, the study was conducted in the Behavioral and Experimental Economics Laboratory (BeeLab) of Maastricht University. Students registered in the BeeLab database were invited to participate. As most registered students were of either German or Dutch nationality, the study was conducted in these two languages. Thus, being Dutch or German was the only inclusion criterion for being invited. In total, 340 students ( $61 \%$ female, mean age $=21$ ) participated in the baseline measurement. At the first follow-up, 240 students participated ( $71 \%$ of baseline, $64 \%$ female, mean age $=21$ ) and a total of 128 students ( $38 \%$ of baseline, $69 \%$ female, mean age $=22$ ) completed the second follow-up, three months after baseline. 


\section{Procedure}

Potential participants registered in the BeeLab database received an invitation email containing the following information: the study aims to gain insight into the relationships of cognitions related to $\mathrm{PA}_{\text {; }}$ it consists of three waves; one measurement is comprised of 2 tasks which together take 30 minutes to complete; there are no expected risks associated with participation; all data will be gathered and analyzed anonymously; participants will receive $15 €$ in cash after the first two waves and another 7,50€ in cash after participation in the third wave. Those willing to participate could select a timeslot from two given days for each wave. One day before participating, a reminder was sent. On the day of participation, participants were welcomed in the Lab, received instructions, and informed consent was obtained from all individuals included in the study. In the first part, participants performed a modified version of the Single-Category Implicit Association Test (SC-IAT) (Karpinski \& Steinman, 2006) to assess implicit attitudes towards PA. In the second part, participants filled in a self-report questionnaire to measure explicit cognitions and PA behavior. Explicit cognitions were assessed subsequently as a prior assessment of explicit cognitions is assumed to trigger thoughts related to PA which in turn might influence the reaction time in a following task (Bargh \& Chartrand, 2000). The SC-IAT and the questionnaire were available in Dutch and in German. After completion participants were thanked and if they took part in follow-ups received their incentive at T1 and T2.

\section{Measurements}

Implicit attitude assessment task. Implicit attitudes towards PA were measured with the SC-IAT. Whereas the IAT relies on the comparison of two opposite categories, e.g. men versus women, the SC-IAT does not. Regarding PA, it is difficult to define a clear opposite category as PA behavior occurs on a continuum. Moreover, the SC-IAT has proved able to predict objectivelymeasured physical activity (Conroy et al., 2010) and unintentional physical activity (Conroy et al., 2010; Hyde et al., 2010). Also, adequate internal reliability and predictive validity were demonstrated (Karpinski \& Steinman, 2006).

The SC-IAT consisted of two blocks, each comprising 24 practice trials and 72 test trials. In one block, "physical activity or positive" formed one category and "negative" the other category. In the other block, "physical activity or negative" was one category and "positive" the other. It is assumed that a person possesses a positive implicit attitude when he or she is quicker to categorize the displayed stimuli when "physical activity or positive" form one category than when "physical activity or negative" are one category. When this pattern is reversed, the person is assumed to hold a negative implicit attitude. The order of the two blocks was counterbalanced, meaning that the block "physical activity or positive" and "negative" had to be performed first by some participants, whereas other participants performed the block "physical activity or negative" and "positive" first. Labels for the two categories were presented on either the left or right upper part of the screen throughout the task. One by one, stimuli were presented in the centre of the screen and participants had to press $e$ on their keyboard when the stimulus belonged to the category presented on the left or $i$ when the stimulus 
belonged to the category displayed on the right. The sequence in which the stimuli were presented was randomized and words appeared an equal number of times. When an incorrect answer was given, a red $\mathrm{X}$ appeared on the screen until a correct answer was given.

Positive and negative words were selected from the Affective Norms for English Words (ANEW) (Bradley \& Lang, 1999) based on their valence and arousal norms. The words were translated to and from Dutch and German by German and Dutch native-speaking researchers of Maastricht University. In an informal pretest, 26 German and 22 Dutch students of Maastricht University rated the words with regard to the perceived levels of valence ( 1 = very negative to 9 = very positive), arousal ( $1=$ not arousing at all to $9=$ very arousing), and familiarity ( 1 = very unfamiliar to $9=$ very familiar) in their respective mother tongue. On this basis, the following positive words were selected: love, freedom, joy, success and party (translated from German and Dutch). The selected negative words were: depression, demon, lie, infection, and poison (translated from German and Dutch). Words representing PA were carefully chosen from earlier studies in which the SC-IAT was used to assess implicit attitudes towards PA (Conroy et al., 2010; Hyde et al., 2010). These were also translated to and from German and Dutch and pretested for their representativeness for PA in both languages ( $1=$ not representative at all, 2 = not very strongly/moderately representative, 3 = strongly representative). The seven words that were highly representative for PA were: running, biking, kickboxing, sprinting, jogging, weight-lifting, and (doing) sit-ups (translated from German and Dutch).

The SC-IAT was programmed using Inquisit by Millisecond software and the script was based on Karpinski and Steinman (Karpinski \& Steinman, 2006). The implicit attitude was formed by d-scores, calculated automatically using Inquisit software by subtracting the average response time for the test block with the categories physical activity or positive/ negative from the average response time of the test block with the categories physical activity or negative/positive. This score was then divided by the standard deviation of all test trials. This procedure is based on the improved scoring algorithm as described by Greenwald and colleagues (Greenwald, Nosek, \& Banaji, 2003). D-scores can range from -2 to +2 with negative values representing a negative implicit attitude and positive values representing a positive implicit attitude. The higher the $d$-score the more positive an implicit attitude. Reliability test of the SC-IAT was calculated based on the procedure as described in Karpinski and Steinman (2006) and revealed an acceptable value of $r=.83$.

Self-report assessment. All explicit cognitions referred to adequate physical activity. Adequate PA for adults was defined as being moderately physically active five times a week for at least 30 minutes. Moderately active is described as, for instance, brisk walking with an increase in heart rate (Health \& Services, 2008). This definition was presented to the participants and could be re-read at any time while answering the questionnaire. The questions to measure explicit cognitions were based on the I-change model (De Vries et al., 2005).

Explicit attitude was assessed using 20 items that were rated on a 5-point Likert Scale. Ten items assessed the pros of adequate PA (Cronbach's $\alpha=.83$ ) and 10 items assessed the perceived disadvantages of adequate PA (Cronbach's $\alpha=.77$ ). One example item for pros is 
"When I am adequately active it is" with answer options ranging from (1) "very good for my health" to (5) "not good for my health". Items were reversed so that higher values represent the perception of more pros. An example for cons is "When I am adequately active it is" with answer options from (1) "too time-consuming" to (5) "not time-consuming". Items were reversed, so that lower scores represent the perception of fewer cons. One scale score for perceived pros and one for perceived cons were created for the analyses.

Social norms and social modeling were assessed by four questions. Answers were given on a 5-point Likert scale and assessed the norms about adequate physical activity of family members, partners, and friends (Cronbach's $\alpha=.74$ ) and their PA behavior (Cronbach's $\alpha$ $=.48$ ). An item representing norms was "Most of my friends" (1) "certainly think that I need to be adequately active" to (5) "certainly do not think that I should be adequately active". An additional answer option: "I don't have any friends/Not applicable" was given as a sixth option. A modeling item was "Most of my friends are adequately physically active" with answer options from (1) "totally agree" to (5) "totally disagree". The additional answer option "I don't have any friends/Not applicable" was also available. These answers were not included in the analyses. Norms and modeling items were reversed with higher scores representing stronger norms or modeling. The mean scale scores for norms and modeling were included in the analyses.

Self-efficacy was measured by nine items, also on a 5-point Likert scale (Cronbach's $\alpha$ $=.74$ ). These items enquired about the extent to which respondents thought they would be able to be adequately physically active in different situations. For instance, "I find it difficult/ easy to be adequately physically active when I am tired" with answer options from (1) "very difficult" to (5) "very easy". Questions were based on those used in former studies about PA (Schulz et al., 2014; Schulz et al., 2012; Smeets, Brug, \& De Vries, 2006). Higher scores indicate higher self-efficacy. The mean scale score was included in the analyses.

Intention was measured by three items on a 5-point Likert scale (Cronbach's $\alpha=.87$ ). The first item assessed whether respondents intended to become adequately physically active within the next three months, ranging from (1) "yes, absolutely" to (5) "no, not at all". The second item asked whether respondents were motivated to become adequately physically active within the next three months with answer options ranging from (1) "totally agree" to (5) "totally disagree". The third item measured how high the chances were of becoming adequately physically active within the next three months. Answer options ranged from (1) "very little" to (5) "very high". The first two items were reversed, so that higher scores represent a stronger intention. The mean score of all three items was included as scale score for intention in the analyses.

Physical activity levels were measured by the Short Questionnaire to Assess Healthenhancing physical activity (SQUASH). This has been proven to be a reliable and valid tool for assessing PA levels among Dutch adults (RIVM, 2005; Wendel-Vos, Schuit, Saris, \& Kromhout, 2003) and has been applied in former studies (De Vries et al., 2008; Schulz et al., 2014; Smeets et al., 2006; Smeets et al., 2007). Completing the SQUASH takes around five minutes; it assesses 
different domains of PA, namely commuting activities, activities at work, household activities, and leisure time activities. For each activity, frequency (days per week), duration (minutes per day) and intensity (light/moderate/intense expressed in metabolic equivalent of task, MET) were measured. MET values for sport activities were derived from Ainsworth and colleagues (1993). Based on the procedure of Wendel-Vos and colleagues (2003), the total minutes of an activity were calculated by multiplying frequency by duration. These were then multiplied by the intensity in order to obtain an activity score for each activity. A total activity score was calculated by summing all activity scores. The higher the score, the more physically active a person is.

Additionally, participants gave information about their age, gender, use of drugs, alcohol or medications that could influence their reaction time, and whether they were able to be physically active in the recent past.

\section{Analyses}

Differences between the German and Dutch version of the tests were tested in advance. No significant differences were found. Descriptive analyses were conducted to describe the sample. To assess whether study variables changed significantly over time, linear mixed models were used, the recommended method for repeated measures with missing data. Logistic regression analysis was used to evaluate whether dropout was predicted by age, gender, perceived pros, perceived cons, social norms, social modeling, self-efficacy. All analyses were done with SPSS version 23.

For the first hypothesis, two hierarchical multiple regressions were performed: one with PA behavior after one month, and a second with PA behavior after three months as dependent variable. Baseline variables were included as predictors in three steps. In step 1 we entered age and gender, in step 2 perceived pros, perceived cons, social norms, social modeling, selfefficacy and intention, and in step 3 implicit attitudes as predictor. For hypothesis 2, there was a fourth step, entering all interaction terms between implicit attitude and the explicit cognitions. If there were significant interaction terms, follow-up stratified analyses were conducted (Aiken, West, \& Reno, 1991). In this case, implicit attitude was categorized into positive, neutral, and negative based on the tertiles of its score distribution. Implicit attitude scores $\leq-.053$ were categorized as negative, implicit attitude scores $>-.053$ and $\leq .285$ were considered neutral, and scores $>.285$ as positive. To test whether the interactions found added significantly to the prediction of PA after one month or after three months, another hierarchical regression analysis was performed, only adding the significant interaction terms. To test hypothesis 3 , hierarchical multiple regressions, similar to those carried out for question 2, were performed, but this time with intention at baseline, after one month and after three months as dependent variable. In step 1, we again entered age and gender; in step 2, perceived pros, perceived cons, social norms, social modeling, self-efficacy and implicit attitudes; and in step 3, all interaction terms between implicit attitude and the explicit cognitions. All predictors were mean-centered before entering into the models. Cases with missing values were not included in the analyses. 


\section{RE S U LTS}

\section{Descriptives}

In total, 372 students participated in the baseline measurement. Answers of 32 participants were excluded as their reaction times could not be linked to their questionnaire answers. The remaining sample was $\mathrm{N}=340$ ( $61 \%$ female, mean age $=21)$. Table 2.1 shows the characteristics of the sample and the differences over time regarding study variables. At follow-up one and two, more men dropped out than women $(\mathrm{T} 1: \mathrm{OR}=0.55,95 \% \mathrm{Cl}=0.04-1.0, \mathrm{p}=.02 ; \mathrm{T} 2$ : OR $=0.51,95 \% \mathrm{Cl}=0.02-1.0, \mathrm{p}=.01)$. No other variables predicted dropout.

Table 2.1. Characteristics of the study sample and differences between study variables over time.

\begin{tabular}{lcccccc}
\hline & T0 & T1 & T2 & F value & df & P value \\
& $(\mathrm{N}=340)$ & $(\mathrm{n}=240)$ & $(\mathrm{n}=128)$ & & & \\
\hline Sex (female), $\mathrm{n}(\%)$ & $212(61.1)$ & $165(63.5)$ & $101(70.1)$ & & & \\
Age in years & $21(2.11)$ & $21(2.14)$ & $21(2.19)$ & & 75 \\
Perceived pros & $4.23(.47)$ & $4.29(.46)$ & $4.30(.47)$ & 1.91 & 737 & .15 \\
Perceived cons & $2.00(.50)$ & $2.01(.53)$ & $2.01(.51)$ & .11 & 737 & .89 \\
Social norms & $3.89(.74)$ & $3.90(.74)$ & $4.05(.66)$ & 3.06 & 737 & .05 \\
Social modeling & $3.45(.65)$ & $3.43(.71)$ & $3.46(.73)$ & .10 & 737 & .90 \\
Self-efficacy & $2.60(.62)$ & $2.56(.61)$ & $2.59(.65)$ & .53 & 737 & .59 \\
Implicit attitude & $.116(.331)$ & $.130(.338)$ & $.141(.325)$ & .63 & 737 & .53 \\
Intention & $4.43(.67)$ & $4.38(.70)$ & $4.42(.64)$ & .78 & 737 & .46 \\
Physical activity & $4959.03(3187.16)$ & $5401.21(2980.59)$ & $5593.24(2888.56)$ & 3.32 & 737 & .04 \\
\hline
\end{tabular}

\section{Hypothesis 1}

\section{The contribution of implicit attitudes to the variance in PA behavior}

Implicit attitudes did not add directly to the prediction of PA behavior after 1 month of follow$\operatorname{up}\left(\mathrm{F}_{\text {change }}(1,230=.04, \mathrm{p}=.84)\right.$, nor after 3 months' follow-up $\left(\mathrm{F}_{\text {change }}(1,118)=1.48, \mathrm{p}=.23\right)$. After 1 month, intention ( $t=1.98, p=.05)$ and self-efficacy $(t=2.92, p=.04)$ explained $13 \%$ of the variance in PA behavior, and after 3 months, self-efficacy $(t=2.44, p=.02)$ explained $16 \%$ of the variance in PA behavior.

\section{Hypothesis 2}

\section{Moderating effects of implicit attitudes on the relationship between explicit cognitions and PA behavior}

After one month of follow-up, the effect of self-efficacy on PA behavior was marginally but not significantly moderated by implicit attitudes $(p=.06)$. The positive relationship between selfefficacy and PA was significantly strengthened when people had a positive implicit attitude ( $\beta$ $=.411)$ compared to when the implicit attitude was negative $(\beta=-.040 ; p=.02)$. After three months, implicit attitudes moderated, although only marginally significantly, the relationship 
between intention and PA $(p=.08)$. The relationship was stronger when people held a neutral implicit attitude $(\beta=.376)$ compared to when they held a positive implicit attitude $(\beta=-.296 ; p$ $=.03)$ towards PA. The interaction did not add significantly to the prediction of PA at $T 2\left(\mathrm{~F}_{\text {change }}\right.$ $(1,117)=1.83, p=.18)$. Table 2.2 shows the results for each of the four steps of the hierarchical regression.

\section{Hypothesis 3}

\section{Moderating effects of implicit attitudes on the relationship between explicit cognitions and PA intention}

Interaction effects were found at baseline between perceived cons and implicit attitudes ( $p$ $=.07)$ as well as between self-efficacy and implicit attitudes $(p=.04)$. Table 2.3 presents the results for each of the four steps of the hierarchical regression.

The negative relationship between perceived cons and intention was significantly strengthened when people held a negative implicit attitude $(\beta=-.368)$ compared to when the implicit attitude was positive $(\beta=-.085 ; p=.03)$. The positive relationship between selfefficacy and intention was significantly strengthened when people held a neutral $(\beta=.232)$ or a positive implicit attitude $(\beta=.326)$ compared to when the implicit attitude was negative $(\beta=-.002 ; p=.05, p=.01)$. Along with perceived pros and social modeling, the significant interactions added, although only marginally, significantly to the prediction of intention at baseline $\left(F_{\text {change }}(2,329)=2.63, p=.07\right)$, and explained $42 \%$ of the variance in the intention to become physically active, i.e. $2 \%$ more than without the interactions.

After one month' follow-up an interaction effect between implicit attitudes and social modeling was found $(p=.02)$. The effect was significantly stronger when people held a negative implicit attitude $(\beta=.359)$ compared to when the implicit attitude was positive ( $\beta=.050, p=.06$ ). Along with perceived pros, perceived cons and self-efficacy, the interaction added significantly to the prediction of intention after one month, $\left(F_{\text {change }}(1,231)=5.48\right.$, $p=.02$ ) and explained $32 \%$ of the variance in the intention, i.e. $1 \%$ more.

After three months, implicit attitudes moderated the relationship of social modeling to intention $(p=.03)$. The relationship was, although only marginally significant, stronger when people held a negative $(\beta=.378)$ compared to a positive implicit attitude $(\beta=-.073 ; p=.08$ ) to PA. Along with perceived pros and perceived cons, the interaction between social modeling and implicit attitude significantly added to the prediction of intention after three months $\left(F_{\text {change }}(1,118)=5.08, p=.03\right)$ and explained 39\%, i.e. 3\% more, of the variance in the intention. 


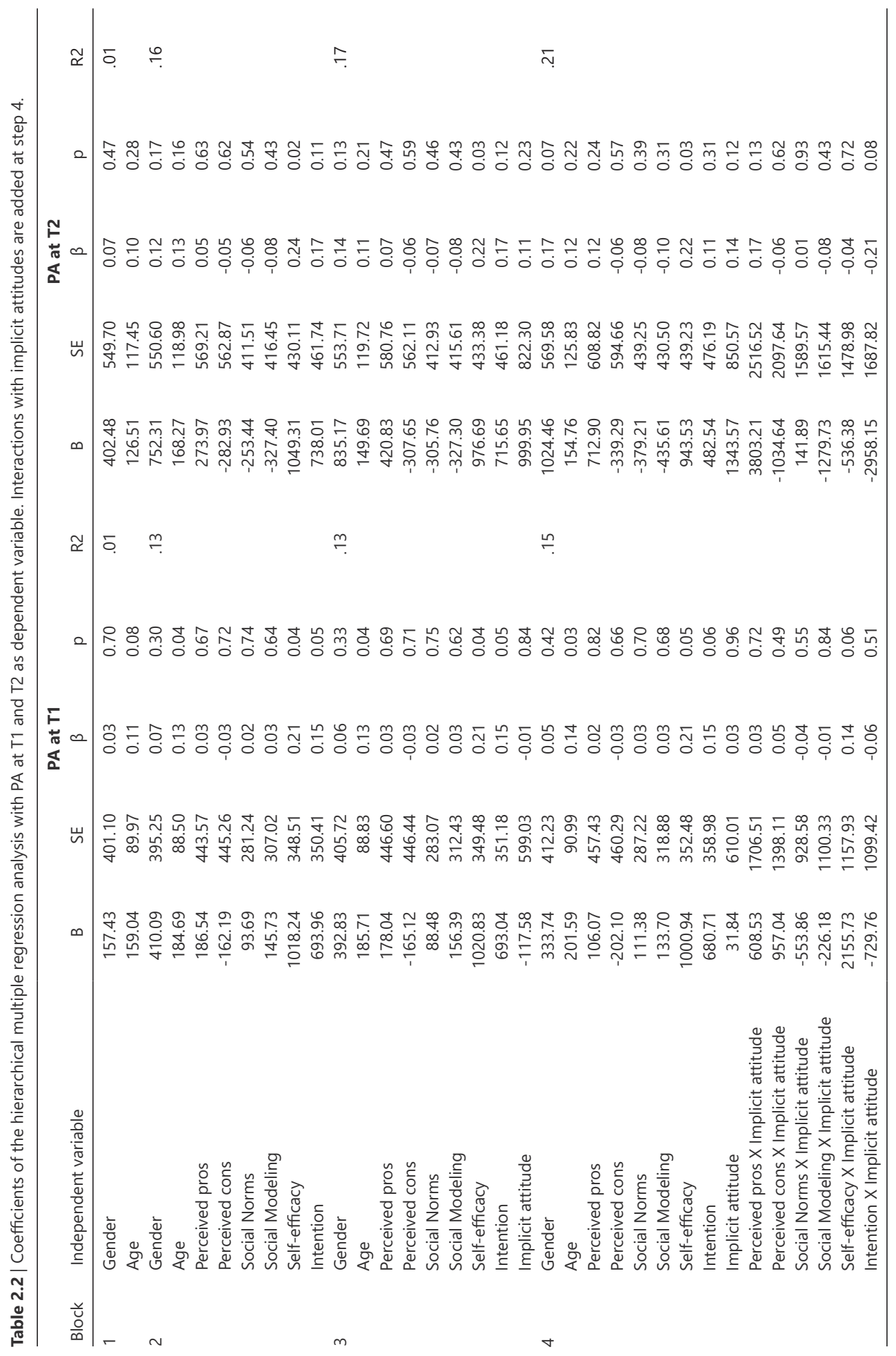




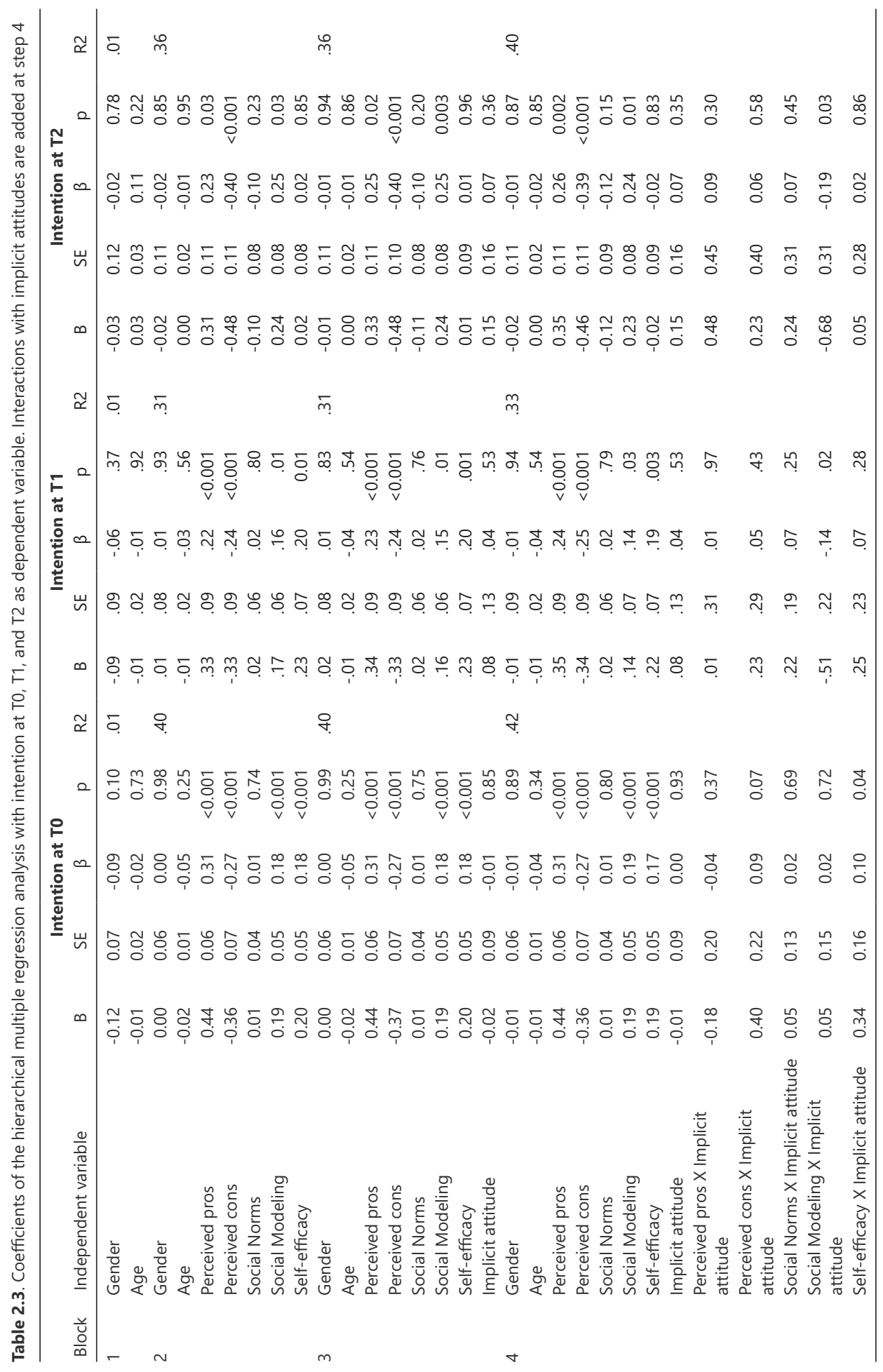




\section{DISCUSSION}

The present study aimed to shed light on the question how implicit attitudes influence PA intention and behavior together with well-known explicit predictors of PA. Direct effects of these variables as well as interactions between them were examined. Results showed that implicit attitudes did not have a direct effect on PA behavior albeit via other explicit cognitions. The fact that implicit attitudes did not have a direct effect on PA behavior at any measuring point is in contrast to our hypothesis as well as to earlier results of Conroy and colleagues (2010) and Cheval and colleagues (2015). Both authors found that, after controlling for explicit motivational predictors, implicit processes significantly contributed to PA prediction and hence support for the additive pattern. Whereas above authors assessed PA behavior using pedometers, we assessed PA levels by means of a self-report questionnaire, which, despite its shown validity (Wendel-Vos et al., 2003), is less accurate than direct measurements (Prince et al., 2008; Slootmaker, Schuit, Chinapaw, Seidell, \& van Mechelen, 2009); this could be a reason for the non-significant findings. Follow-up studies using accelerometers may be needed to obtain further insight into whether or not implicit processes influence actual PA behavior directly.

Although we did not find any direct effects, moderating effects were demonstrated: i.e. positive implicit attitudes strengthened the positive relationship between self-efficacy and PA behavior at the first follow-up. Negative implicit attitudes were found to weaken this relationship. In addition, and similar to Cheval et al. (2015), we found that neutral but not positive implicit attitudes strengthened the positive relationship between intention and PA at the second follow-up. It seems surprising that positive implicit attitudes did not strengthen the relationship between intention and PA, but this could be explained by a ceiling effect as the intention of participants to be active was already very strong. Nonetheless, the findings support the idea of an interactive pattern of influencing PA behavior which is also in line with the findings of Cheval and colleagues (2015). If the intention to be active is already strong, positive implicit attitudes do not seem to support the effect on behavior, whereas neutral implicit attitudes do. In order to strengthen the likelihood that intention translates into behavior, our results suggest that one should at least aim to diminish a negative implicit attitude and create a neutral implicit attitude.

Moreover, we found implicit attitudes moderated the relationship between several explicit cognitions and intention. Firstly, implicit attitudes moderated the relationship between perceived cons and intention as well as between self-efficacy and intention at baseline. In line with our hypothesis, negative implicit attitudes strengthened and positive implicit attitudes weakened the negative relationship between perceived cons and intention. It seems that for those participants who reported exercise not to be beneficial or pleasant (as measured by the explicitly perceived cons), the positive implicit associations with PA acted as a buffer between perceived cons and intention. Moreover, the positive relationship between self-efficacy and intention was strengthened by neutral and positive implicit attitudes. Regarding self-efficacy, 
it seems conceivable that the effect of intention on PA behavior is stronger when a person does not only perceive him or herself as being capable of performing the behavior, but also has a positive, or at least a neutral, unconscious attitude towards the behavior. Thus, when intending to increase PA intention, positive implicit attitudes appear to be more beneficial. The interactions were not significant at one and three months' follow-up, which could either be due to the weaker power of the sample, or to the assumption that implicit attitudes only have a short-term influence on the effect of perceived cons and intention and self-efficacy and intention.

Secondly, at one and three months' follow-up, implicit attitudes moderated the relationship between social modeling and intention. The impact of other people's behavior on the intention to become physically active was significantly greater when the implicit attitude was negative compared to when it was positive. One explanation for this finding could be derived from Festinger's cognitive dissonance theory (1957), according to which, individuals seek consistency among their cognitions. When an inconsistency between attitudes or behaviors occurs, the individual is motivated to resolve it as it is accompanied by negative feelings (Aronson, 1992). Feeling implicitly negative about being physically active while at the same time perceiving important people in one's environment as being physically active, might create dissonance. In order to resolve this, individuals might reduce the importance of the implicit attitude and follow the behavior of others. In this case, the explicitly perceived modeling behavior might override the implicitly perceived negative implicit association. In the present study, the negative implicit attitude had a positive effect on the relationship between social modeling and intention. However, when there is no dissonance, i.e. when a person holds a negative implicit attitude and is surrounded by people who are not sufficiently active, negative implicit attitudes might strengthen the negative relationship between social modeling and intention, as was also the case for the relationship between perceived cons and intention. As interventions may not be able to change or control behavior or the perception of peer or parent behavior, they might rather attempt to reduce the impact of these perceptions on intention by creating a positive implicit attitude. Training or changing implicit associations has been applied to reduce social anxiety (Clerkin \& Teachman, 2010), alcohol consumption (Houben, Nederkoorn, Wiers, \& Jansen, 2011), to increase implicit self-esteem (Ebert, Steffens, von Stulpnagel, \& Jelenec, 2009; Grumm, Nestler, \& von Collani, 2009) and only recently to increase PA levels (Berry, 2016; Markland, Hall, Duncan, \& Simatovic, 2015). While Berry and colleagues and Markland and colleagues demonstrated short-term changes in implicit attitudes via exercise imagery or the provision of (counter attitudinal) information, computerized tasks have not yet been used in this context, but might offer a fruitful alternative. More research is, therefore, needed to understand how stable and changeable implicit attitudes actually are, especially over time. Moreover, in order to understand conditions under which dissonant and congruent implicit and explicit attitudes are beneficial or detrimental for PA behavior, further research is required. 
When interpreting our findings, the following possible limitations need to be taken into account. First, the study sample was quite homogenous as far as age, education, and socioeconomic status were concerned and had, on average, a very positive explicit attitude and a strong intention to be physically activity, which is not representative of the general public (Teixeira, Carraca, Markland, Silva, \& Ryan, 2012). Second, for practical reasons, PA levels were measured by self-report. It is not clear to what extent participants were explicitly aware of activities which occurred spontaneously and excluded planned, structured exercise (e.g. using the stairs), and whether they were able to report them. Despite the satisfactory validity of the SQUASH (Wendel-Vos et al., 2003), supplementing it with more objective measures, such as accelerometers or pedometers, could provide a more adequate report about activity levels as suggested by other studies (Prince et al., 2008; Slootmaker et al., 2009). Third, we had a high drop-out rate at our follow-up measures (29\% at T1 and $62 \%$ at T2) which could be due to an absence of commitment to participate in all three measures, a panel fatigue (Lugtig, 2014) or simply due to time constraints of the student sample as the last measure was conducted shortly before the exam period. As a consequence, our sample suffered from low power after one and three months which makes the interpretation of (non-)findings challenging. Fourth, stratified analyses for people with a negative, neutral, or positive implicit attitude were conducted using small sub-samples; these are also likely to have suffered from low power. Fifth, although neutral or positive implicit attitudes might help to increase PA intention, the intention behavior gap still remains. Research aimed at reducing this gap should be further stimulated.

\section{CONCLUSION}

Summarizing, one can conclude that the present findings challenge the dual-process approach which, until now, only assumed a direct influence of implicit attitudes on behavior, not via other explicit constructs. Although different modes of influence were suggested by Perugini (2005), including an interactive pattern of influence between implicit and explicit attitudes, a thorough examination or integration of further determinants, such as intention, has not yet been carried out. Both approaches have in fact developed in isolation. We argue that this division needs to be reconsidered as our findings and those of Cheval and colleagues (2015) demonstrate that unconscious processes are indeed associated with more conscious processes. A unique contribution of the present research is the examination of interactions between implicit attitudes, which are part of dual-process models, and explicit cognitions, which are summarized in social-cognitive models. Potential improvements for interventions are thus provided. Future research needs to build on these findings by testing whether interventions which target both implicit attitudes and explicit cognitions result in greater activity intention and actual behavior change. Another avenue for future work, especially for the area of model testing and model improvement, is to investigate whether the relationships found in the present study are rather 
unique to PA or valid across diverse health-related behaviors. Shedding light on these issues may not only aid the development of even more successful interventions to promote physical activity, but also the aspiration to improve global health. 
Chapter<smiles>C1CC[Al]C1</smiles> 


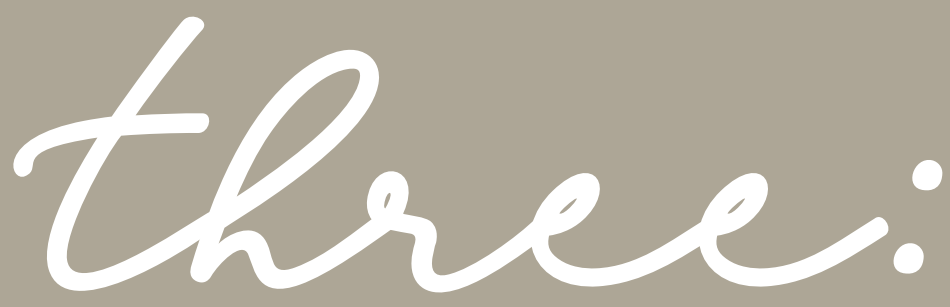

Implicit attitudes and explicit cognitions jointly predict reduced red meat intake: a three-wave longitudinal study

Muschalik, C., Crutzen, R., Candel, M. J.J., Elfeddali, I. \& de Vries, H. (under review). 


\section{A B S T R A C T}

Background: Despite nutritional benefits, a high consumption of red meat (RMC) is not without risks as it is linked to the development of certain types of cancer as well as to other non-communicable diseases, such as type II diabetes or cardiovascular diseases. Moreover, the production of meat has negative effects on the environment. Therefore, a transition to a less meat-based diet could be beneficial. It is unclear how explicit cognitions towards RMC and implicit attitudes jointly influence intention and consumption. We tested the additive pattern (both types of cognitions explain unique variance) and interactive pattern (both types interact in the prediction).

Method: At baseline ( $\mathrm{T} 0 ; \mathrm{N}=1790)$ and one $(\mathrm{T} 1 ; \mathrm{n}=980)$ and three months thereafter ( $\mathrm{T} 2$; $\mathrm{n}=556$ ), explicit cognitions, RMC, and implicit attitudes were assessed among a Dutch sample.

Results: $\quad$ Only explicit cognitions were associated with RMC. Implicit attitudes moderated the effect of self-efficacy on T0-RMC; negative implicit attitudes strengthened this effect. T0-intention was associated with explicit cognitions and implicit attitudes. Additionally, negative implicit attitudes strengthened the effect of social norms on T0 and T2-intention. Regarding RMC, support for the interactive pattern was found. For intention there was support for the interactive and additive pattern.

Conclusion: Interventions aiming to reduce red meat consumption in the general public might profit from changing implicit attitudes in addition to explicit cognitions. 


\section{INTRODUCTION}

Red meat (RM) can be an important dietary source of protein and essential nutrients, such as iron, zinc and vitamin B12 (Chan, McCance, \& Brown, 1996; Johnston, Prynne, Stephen, \& Wadsworth, 2007). However, when consumed excessively, it can also be a threat to people's health as it is associated with the development of several diseases, e.g. colon and lung cancer (Cross et al., 2007; Giovannucci et al., 1994), cardiovascular diseases (Kelemen, Kushi, Jacobs, \& Cerhan, 2005; Kontogianni, Panagiotakos, Pitsavos, Chrysohoou, \& Stefanadis, 2008; Sinha, Cross, Graubard, Leitzmann, \& Schatzkin, 2009), type II diabetes (Pan et al., 2011; Song, Manson, Buring, \& Liu, 2004), obesity (Pan et al., 2012), and increased mortality in general (Sinha et al., 2009). In 2015, this led the World Health Organization (WHO) to classify the consumption of processed RM - referring to meat preserved by smoking, curing or salting, or the addition of chemical preservatives, including that contained in processed foods - as carcinogenic and the consumption of RM - speaking of beef, pork, lamb, and goat from domesticated animals including that contained in processed foods - as potentially carcinogenic (Bouvard et al., 2015; McGuire, 2016). The individual recommendation for people who eat red meat is to reduce their intake to no more than $500 \mathrm{~g}$ a week of red meat (equiv. $26 \mathrm{~kg} / \mathrm{year}$ ) and very little if any processed red meat. The public health goal for the population average consumption of red meat should be no more than $300 \mathrm{~g}$ a week (equiv. $14.4 \mathrm{~kg} / \mathrm{year}$ ) and very little if any of which to be processed (Marmot et al., 2007). With an annual average consumption of 43.4 $\mathrm{kg}$ per person, European citizens exceed this recommendation by far (Chemnitz \& Becheva, 2014). Dutch citizens consume far more RM than recommended: in 2017 they ate on average $54.6 \mathrm{~kg}$ of red and processed red meat per person and year, which does not even take into account the amount of RM entailed in ready-made meals. Furthermore, meat consumption does not only have negative effects on people's health, but also on the health of animals and the planet (Aiking, 2014; Friel et al., 2009; Steinfeld, Gerber, Wassenaar, Castel, \& De Haan, 2006; Westhoek et al., 2014). Hence, a transition to a less meat-based diet could be beneficial (Scott, 2017). As a natural decrease in RM intake is not expected in the near future (Chemnitz $\&$ Becheva, 2014), a deeper understanding of the cognitions that determine a moderate RM intake is needed in order to inform future intervention efforts.

In order to explain health behaviors, two approaches can be chosen which are not necessarily mutually exclusive. Social cognitive models, which are the more traditional approach, focus on explicit, deliberate, and volitional constructs. These models explain an individual's intention and behavior by (beliefs underlying) determinants that people can reflect on and can express consciously (i.e., they are reasoned, but not necessarily rational). These determinants are, for example, explicit attitude, self-efficacy or social norms. Another approach focuses on unconscious and more automatic associations that are less subject to reflection by an individual, meaning that they occur regardless of whether the individual perceives them as valid or invalid (Gawronski \& Bodenhausen, 2006). As human behavior is probably neither solely consciously nor unconsciously regulated (Vrabel \& Zeigler-Hill, 2017), 
we argue that in order to understand the consumption of red meat, it is important to take both conscious and unconscious constructs into account. Until now, constructs derived from both approaches have been examined in a few studies on meat intake, however, as far as we know only in isolation from each other (Barnes-Holmes, Murtagh, Barnes-Holmes, \& Stewart, 2010; Berndsen \& van der Pligt, 2005; Carfora, Caso, \& Conner, 2017a; De Houwer \& De Bruycker, 2007; Graça, Calheiros, \& Oliveira, 2015; Lea \& Worsley, 2001). The aim of the study at hand is therefore to investigate how conscious and unconscious constructs together predict a (reduced) red meat intake.

According to social cognitive models, such as the Reasoned Action Approach (Fishbein \& Ajzen, 2011) or the I-change model which integrates constructs from various social cognitive models (De Vries, 2017; De Vries et al., 2005), an individual's intention is the most proximal cause for behavior. Intention in turn is formed by three key determinants: (1) a person's explicit attitude towards the behavior (comprised of perceived pros and perceived cons, i.e. the perceived advantages and disadvantages the behavior entails), (2) social influence (comprised of social modeling and social norms, i.e. how people in one's environment behave and the perceived social pressure to perform a behavior) and (3) self-efficacy (i.e. the perceived ability or difficulty of performing the given behavior). These constructs were indeed strongly associated with reduced (red) meat intake. That is, positive explicit attitudes towards reducing red meat consumption increased the intention to reduce one's intake (Carfora et al., 2017a), whereas positive explicit attitudes towards meat decreased the intention (Graça et al., 2015). Moreover perceiving oneself as capable of changing one's meat consumption (i.e. selfefficacy) predicted the intention to reduce one's consumption (Carfora et al., 2017a; Graça et al., 2015) and a higher number of vegetarian friends (social modeling) is inversely related to an individual's red meat consumption (Lea \& Worsley, 2001). A high intention, in turn, has been shown to result in a lower actual intake of meat (Berndsen \& van der Pligt, 2005; Carfora et al., 2017a).

The contemporary approach of combining implicit and explicit cognitions as determinants of behavior is depicted in dual process models (Hofmann et al., 2008; Sheeran et al., 2013; Strack \& Deutsch, 2004; Wilson et al., 2000). The Reflexive-Impulsive Model (RIM) (Strack \& Deutsch, 2004) is one example of a dual-process model, which distinguishes between a reflective and an impulsive system. The impulsive system is defined as composition of automatic behavioral tendencies and the reflective system is understood as the composition of reasoned, deliberate, and conscious motives to pursue a behavior. According to Perugini (2005) there exist three possible ways in which the impulsive and reflexive system can operate in guiding a behavior: (1) both systems explain unique variance in the behavior (additive pattern), (2) the impulsive system predicts spontaneous and the reflective system predicts deliberate behavior but not vice versa (double dissociation pattern) and (3) both types of systems interact synergistically in the prediction of behavior (interactive pattern), i.e. one type of determinant strengthens or weakens the effect of the other type of determinant on behavior. Implicit attitudes are one type of implicit (or impulsive) processes that are activated automatically and occur partially 
or completely outside a person's awareness (Rydell \& McConnell, 2006). Implicit attitudes are clearly distinguished from the abovementioned explicit attitudes that are incorporated in the social-cognitive models and are commonly assessed by means of self-reported questionnaires. Implicit attitudes, on the contrary, are inferred by computerized reaction time tasks, of which the Implicit Association Test (IAT) (Greenwald et al., 1998) is the most used one. Briefly, the IAT measures the relative strength of attitudes towards opposing targets (e.g. men vs. women or black vs. white). In order to measure absolute attitudinal strength (e.g. when the question involves predicting responses to one specific target or when a natural opposing target is not identifiable), the Single-Category Implicit Associations Task (SC-IAT) (Karpinski \& Steinman, 2006) was designed. Contrary to the IAT, it requires only one target concept (e.g. meat), and one target attribute (e.g. negative) and a contrast attribute (e.g. positive), which are represented by stimuli that participants have to sort as quickly as possible to given categories. The idea underlying this method is that respondents are more likely to react quickly when the concept and the attribute are closely associated in memory (e.g. meat and positive) and more slowly when the concept and the attribute are not or less associated with each other (e.g. meat and negative). Based on the performance, the participant's implicit attitude is inferred. Implicit attitudes are correlated with food intake in general (Conner, Perugini, O'Gorman, Ayres, \& Prestwich, 2007; Friese, Hofmann, \& Wänke, 2008; Maison, Greenwald, \& Bruin, 2001). Also in the context of (red) meat consumption, implicit attitudes have been demonstrated to be crucial. That is, meat eaters showed a less positive implicit attitude for vegetables relative to meat compared to vegetarians (De Houwer \& De Bruycker, 2007) and they also indicated a small pro-meat tendency compared to vegetarians (Barnes-Holmes et al., 2010).

Previous work has demonstrated that both implicit and explicit cognitions play a role in (reduced) red meat intake, but studies focusing on the mode of operation between them resulted, depending on the target behavior, in mixed findings. A study on snack versus fruit intake supported the double dissociation pattern and a study with smoking as target behavior supported the interactive pattern (Perugini, 2005). Another study on the behavioral choice of fruits or snacks found support for the additive pattern (Richetin, Perugini, Prestwich, \& O'Gorman, 2007) and studies regarding physical activity found support for the additive pattern (Bluemke et al., 2010; Calitri et al., 2009; Conroy et al., 2010) as well as for the interactive pattern (Cheval et al., 2015; Muschalik et al., 2018; Perugini, 2005). These findings do not only demonstrate that the mode of operation does not only depend on the target behavior but also that implicit and explicit cognitions can regulate one and the same behavior in accordance with an additive and an interactive pattern. This seems logical as both patterns do not necessarily exclude each other: implicit attitudes and explicit determinants could have a direct effect on behavior (suggesting an additive pattern) and also interact with each other (suggesting an interactive pattern). Therefore, we expect that also in the context of red meat consumption, behavior can be directly influenced by both types of cognitions (additive pattern) and that both types of cognitions can interact and either reinforce or weaken each other (interactive pattern). 
Additionally, we argue that it is important to not only shed light on behavior but also on the intention to reduce one's red meat intake. As displayed in the social-cognitive models, intention is understood as the most proximate determinant for behavior and thereby an important prerequisite for behavioral purposes. Hence, understanding the process of intention formation would be a first step in the direction of behavioral change. In a study of Muschalik et al. (2018), implicit attitudes moderated the effect of certain explicit cognitions (e.g. perceived pros, social modeling, self-efficacy) on the intention to be physically active and we expect these results to be transferrable to the context of a reduced red meat intake. That is, the positive effect of perceived pros on the intention to reduce one's intake is expected to be reinforced by negative implicit attitudes towards red meat whereas positive implicit attitudes towards red meat are assumed to weaken the same relation. The same reasoning could be applied to other predictors of intention such as self-efficacy for instance: the positive effect of self-efficacy on intention could be reinforced by negative implicit attitudes towards red meat but weakened by positive implicit attitudes. Furthermore, a few studies have investigated the direct effect of implicit processes or attitudes, respectively, on intention. For example, Dal Cin et al. (2007) revealed that after exposure to a movie in which the protagonist smoked, selfsmoking associations as measured by the IAT, were increased which in turn predicted changes in the intention to smoke. Additionally, von Hippel et al. (2008) provided evidence that nurses' implicitly measured prejudice toward injecting drug users predicted unique variance in the behavioral intention to change jobs. Brochu and Morrison (2007) showed that implicit weight biases predicted participants' behavioral intentions to interact socially with an overweight male person. Based on the findings that implicit processes, such as implicit attitudes, have a direct effect on intention besides explicit predictors, we expect that implicit attitudes also have a direct effect on the intention to reduce red meat intake besides explicit cognitions and will therefore investigate this hypothesis in addition. Given the fact that a single behavior was considered, the double dissociation pattern could not be tested.

Hence, the aim of the study was fourfold. First, we investigated whether implicit attitudes and explicit cognitions predict (a reduced) red meat intake separately from each other (additive pattern). Secondly, we explored if implicit attitudes and explicit cognitions interact in the prediction of (a reduced) red meat consumption (interactive pattern). Third, we assessed if implicit attitudes interact with explicit cognitions (perceived pros, perceived cons, social norms, social modeling, self-efficacy) in the short and long-term prediction of the intention to reduce one's red meat intake (interactive pattern) and fourth, if implicit attitudes also have a direct effect on intention in the short-term as well as long-term (additive pattern). 


\section{METHOD}

\section{Design}

A three-wave longitudinal study was conducted with a baseline measurement (T0), a follow-up after one month (T1) and another follow-up after three months (T2). We have preregistered the study protocol at https://osf.io/vrdqw/register/565fb3678c5e4a66b5582f67?view_ only=bc77614ed5154078b43cf9474aa2a9c3 before data collection. Furthermore, materials used in this study as well as non-identifiable data, syntax, and output of the analyses are available at https://osf.io/7enj9/. These efforts are taken to acknowledge a call for full disclosure to maximize scrutiny, foster accurate replication, and facilitate future data syntheses (e.g., meta-analyses) (Peters, Abraham, \& Crutzen, 2012).

\section{Ethical approval}

Ethical approval was obtained from the FHMLRec at Maastricht University, the Netherlands (Muschalik/220517).

\section{Power analysis}

To determine the sample size, a power analysis was conducted using G*Power. Anticipating a small effect size $\left(f^{2}=0.03\right)$ for a main effect or interaction effect of implicit attitude and the test power set at 0.80 with a type I error rate of $\alpha=0.05$ for two-sided testing, the calculation revealed that a minimum sample of 488 is required. Based on former experiences of the internet research agencies that we collaborated with in this study, a drop-out rate of $60 \%$ between the baseline and the second follow-up (T2) was estimated. Hence, we aimed to have data of 1220 participants available at the first measurement (after having implemented various exclusions) in order to have data of 488 participants available at the second follow-up.

\section{Procedure}

Participants were recruited among members of two internet research agencies. Individuals were eligible to participate in the study when they were older than 18 years and had indicated earlier that they consume meat regularly. Participants who met the inclusion criteria, were invited by email. When willing to participate, they received the participants' information explaining that the study aims to gain insight into the relationship between determinants related to eating behavior and that there would be three measurements. Further, they were informed that one measurement would take 15-20 minutes to complete, that each measurement entailed a reaction time task and a questionnaire, that no risks are related to the participation, that all data would be gathered and analyzed anonymously, and that they would receive a monetary reward for their participation. Depending on the standards for payment of the two different Internet panels, participants received $€ 2.50$ or $€ 4.75$ for participation in the baseline, $€ 2.50$ or $€ 3.00$ for participation in $\mathrm{T} 1$, and $€ 4.00$ or $€ 5.00$ for participation in T2. In order to begin with the study, an electronic informed consent needed to be read and agreed 
upon. If participants did not provide consent for participation, they were excluded from the study. In order to double-check whether only people who consumed red meat at least once a month participated, we included a question on this in the beginning. People who answered the question with "no" were excluded from further participation. In the first part of the study, we assessed participants' implicit attitudes towards red meat by means of a modified version of the Single-Category Implicit Association Test (SC-IAT) (Karpinski \& Steinman, 2006). Afterwards, they filled in a questionnaire to measure explicit cognitions towards RMC (explicit attitude comprised of perceived pros and perceived cons, social modeling, social norms, self-efficacy, intention to reduce RMC) and red meat consumption. The participants had to perform the SC-IAT first, as a prior assessment of explicit cognitions might trigger red meat related thoughts which would then in turn influence the reaction time in a following task (Bargh \& Chartrand, 2000). One and three months after baseline, a new invitation was sent automatically to only those participants who had participated in the previous measure and were invited to complete the follow-up (e.g. T0 participants, who had not completed the T1 measurement, were not invited to participate at the T2 measurement). At both follow-ups, again participants' implicit attitude towards red meat as well as all abovementioned explicit cognitions and self-reported RMC were assessed.

\section{Measurements}

Implicit attitude assessment task. In order to assess implicit attitudes towards red meat, we used the SC-IAT for which satisfactory internal consistency has been demonstrated (Karpinski \& Steinman, 2006). Since the IAT is based on comparisons between concepts, it always measures the association of attributes of one concept relative to another. As we were interested in the implicit attitudes towards red meat unrelated to an opposed category, the SC-IAT was chosen as it measures associations with a single category. As evaluative stimuli we used positive and negative words from the Affective Norms for English Words (ANEW) (Bradley \& Lang, 1999) which were translated forth and back from English to Dutch by Dutch native researchers of Maastricht University. The Dutch words were then pretested regarding their perceived levels of valence ( 1 = 'very negative' to 9 = 'very positive'), arousal ( $1=$ 'not arousing at all' to 9 = 'very arousing'), and familiarity ( 1 = 'very unfamiliar' to 9 = 'very familiar') by a sample of 28 people. Words with the highest scores regarding positivity and familiarity and similar arousal levels were selected as positive stimuli (love, friend, freedom, humor, joy; translated from Dutch). Words with the lowest scores on positivity, highest scores of familiarity and similar evaluations of arousal were selected as negative stimuli (death, hate, devil, loneliness, lie; translated from Dutch). To represent red meat, we selected pictures that were used in the study of De Houwer and De Bruycker (2007) and from the Internet which were free to be used (Creative Commons Images). These were pretested regarding their representativeness for red meat $(1=$ 'not representative at all', 2 = 'not so strongly/a bit representative', 3 = 'strongly representative'). Based on this, seven pictures which were identified as the most representative for red meat were included in the SC-IAT. 
The SC-IAT was programmed by using the software Inquisit by Millisecond (Version 4) and the script was based on Karpinski and Steinman (2006). The SC-IAT contained two blocks which each consisted of 24 practice trials and 72 test trials. In one block "red meat or positive" versus "negative" built the two categories, in the reversed block "red meat or negative" versus "positive" were the two categories. One after one, pictures of red meat and negative or positive words appeared in the middle of the screen. Participants were instructed to indicate as rapidly as possible to which of the two categories the stimulus belonged. The two blocks were presented in a counterbalanced order, thus some participants received the block "red meat or positive" versus "negative" first and the reversed one subsequently whereas other had the block "red meat or negative" versus "positive" first and the reversed one afterwards. The idea underlying the SC-IAT is that when a person is quicker with categorizing the stimuli when "red meat or positive" built one category than when "red meat or negative" are one, the person's implicit attitudes towards red meat is positive and vice versa. Throughout the task, category labels were displayed on the left and right upper part of the screen. When a presented stimulus belonged to the category displayed on the left upper part of the screen, participants had to press $e$ on their keyboard. When the stimulus belonged to the category shown on the right upper part of the screen, they had to press $i$. All stimuli were presented in a randomized order and equally frequent. If an incorrect answer was given, a red $\mathrm{X}$ appeared on the screen until the answer was corrected.

The implicit attitude was indicated by $d$-scores. The $d$-score was calculated automatically by the Inquisit software using the D-algorithm proposed by Greenwald et al. (2003) with more positive values indicating a more positive reaction to red meat. D-scores can range from -2 to 2 and everyone in our sample scored between this range. After the SC-IAT, participants were asked whether they were distracted while performing the task, stating different types of distraction they could select (e.g. 'I was talking on the phone', 'I was eating or drinking', 'I was listening to music' etc.). Only when participants selected 'I was not distracted', their d-score was included in the analyses. To assess the internal reliability of the SC-IAT, the SC-IAT was divided into thirds (blocks of 24 test trials) and a SC-IAT score for each third was calculated (Karpinski \& Steinman, 2006). The average intercorrelation among these scores was identified by means of the Spearman-Brown formula. This adjusted reliability coefficient is conceptually equivalent and directly comparable to Cronbach's alpha. With a value of $r=.73$, the internal consistency was deemed acceptable.

Self-report assessment. The formulations of questions to measure explicit cognitions related to red meat intake were based on the I-change model (De Vries, 2017; De Vries et al., 2005), which has previously been used to identify eating related cognitions (Schulz et al., 2014; Walthouwer, Oenema, Candel, Lechner, \& de Vries, 2015). The questionnaire can be found at https://osf.io/7enj9/?view_only=d1afaf26fdbe4f13a9feb0d857c89db0.

Explicit attitude was assessed with two scales measuring the perceived pros and perceived cons of red meat consumption, which address underlying beliefs of the behavior. The content for the beliefs regarding meat consumption were derived from earlier studies (Dibb 
\& Fitzpatrick, 2014; Verbeke \& Viaene, 1999). Perceived pros and perceived cons were each expressed by 10 statements on a 5 -point Likert Scale. One example for pros is "Eating red meat is" (1) "not tasty" to (5) "very tasty". Due to low factor loadings, two perceived pros items were removed and a mean scale score was created of the remaining eight items and included in the analyses $(\Omega=.73)$. Higher values represent perceiving more pros. An example for cons is "Eating red meat is" (1) "not unhealthy" to (5) "very unhealthy". One item had a low factor loading and was also removed. A mean scale score was created of the remaining nine items and included in the analyses $(\Omega=.66)$. A lower score represents perceiving fewer cons.

Social norms and social modeling were each assessed by four items. On a 5-point Likert scale, norms of family members, partners, and friends regarding reducing red meat consumption were assessed as well as their behavior. A norm item was "Most members of my family" (1) "don't think that I have to reduce my red meat intake" to (5) "certainly think that I have to reduce my red meat intake". A modeling item asked "How many of your family members consume red meat?" with answers ranging from (1) "None of them" to (5) "All of them" or "My partner eats red meat" with (1) "Yes", (2) "No", and (3) "I don't have a partner/not applicable" as answer options. We included a mean scale score for norms $(\Omega=.81)$ in the analyses and all four social modeling items were entered separately as latter construct showed a low internal structure $(\Omega=.20)$. Higher scores represent stronger norms or modeling.

Self-efficacy was assessed by nine items and was based on perceived barriers to reduce one's meat intake (Dibb \& Fitzpatrick, 2014). These items asked participants on a 5-point Likert scale to indicate to what extent they perceive themselves as capable of reducing their red meat intake, for instance "I will be able to reduce my red meat consumption even when I am used to eat red meat" with answer options from (1) "completely disagree" to (5) "completely agree". A mean scale score was included in the analyses $(\Omega=.74)$. Higher scores indicate higher levels of self-efficacy.

Intention was measured by three items. The first item assessed whether respondents were planning to reduce their red meat intake, with answer options ranging from (1) "No, I am not planning to reduce my red meat intake" to (7) "Yes, within one month". The second item (likeliness to change) asked to indicate how likely it was that the person would reduce his/her red meat intake within the next three months, with answers from (1) "very unlikely" to (5) "very likely". The third item (intention strength) assessed how strongly the person was planning to reduce his/her red meat intake within the next three months. Answer options ranged from (1) "very little" to (10) "very strongly". Intention items were entered separately in the analyses as factor saturation of the standardised sum scores was estimated as insufficient $(\Omega=.07)$. Higher scores on all items represent a stronger intention.

Based on former diet-related studies (Springvloet, Lechner, Candel, De Vries, \& Oenema, 2016; Van Assema, Brug, Ronda, Steenhuis, \& Oenema, 2002) and the Food Frequency Questionnaire (FFQ), we assess red meat consumption (RMC) by means of two items. Participants were asked on how many days per week they usually consume red meat (ranging from 1-7 days per week and the additional answer option 'Not on a daily basis but at least 
once a month') and how many grams they usually consume on these days (open question). To provide a reference point, we added the information that a piece of prepared meat at the main meal equals $100 \mathrm{gr}$ and a slice of meat topping (e.g. ham) equals $15 \mathrm{gr}$. The weekly RMC was calculated by multiplying the frequency by the amount of grams and was used in the analyses.

Further we assessed participants age ('What is your age'), sex ('What is your gender?') and level of education, which were used as cofounders in the analyses. Also we added two control questions (e.g. 'From the following answer options, please select statement 4') and excluded data of those participants who did not answer the control questions correctly.

\section{Analyses}

To assess the scale quality of the measurements that were used in the present study, we first calculated their dimensionality by means of exploratory factor analyses. Subsequently, McDonald's (2013) omega was calculated as a less biased alternative to Cronbach's alpha (Crutzen \& Peters, 2017). Compared to alpha, omega reduces the risks of under- and overestimation of internal consistency (Dunn, Baguley, \& Brunsden, 2014) and has more realistic assumptions regarding variances of and covariance between items (Peters, 2014). Omega $_{\text {hierarchical }}$ is based upon the sum of the squared loadings of items on the general factor. Values were calculated with R Studio and were presented in the measurements section above. All other statistical analyses were conducted using SPSS (IBM) version 24. Logistic regressions were used to investigate whether dropout at $\mathrm{T} 1$ and $\mathrm{T} 2$ was predicted by the variables age, gender, education, perceived pros, perceived cons, social modeling and social norms, selfefficacy, intention, and RMC.

To answer the first research question, we performed three hierarchical multiple regressions, with the first regression having RMC at baseline as dependent variable to investigate crosssectional effects. In the second regression, we used RMC after one month as dependent variable, and in the third one RMC after three months as dependent variable in order to assess long-term effects. In all three regressions, baseline variables were added as predictors in three steps. In step 1 we entered age, gender, and education, in step 2 perceived pros, perceived cons, social norms, social modeling, self-efficacy and intention, and in step 3 implicit attitudes as predictor.

To answer the second question, we added a fourth step to the abovementioned regressions, in which we entered all interaction terms between implicit attitude and the explicit cognitions. In case significant interaction terms were found, follow-up stratified analyses were conducted (Aiken et al., 1991). In this case, implicit attitude was categorised into positive, neutral, and negative based on the tertiles of its score distribution. Implicit attitude scores $\leq-.167$ were categorized as negative, scores $>-.167$ and $\leq .103$ were considered neutral, and scores $>.103$ as positive implicit attitudes. In order to investigate whether the found interactions added significantly to the prediction of RMC after one month or after three months, we performed another hierarchical regression analysis, only with the addition of the significant interaction terms. 
To test the third and fourth questions, we performed hierarchical multiple regressions, similar to those carried out for question 2, but this time with intention each at baseline, after one month and after three months as dependent variable. In the first step, age, gender and education were entered; in the second step, baseline perceived pros, perceived cons, social norms, social modeling, and self-efficacy, implicit attitudes in a third step and in step 4, all interaction terms between implicit attitude and the explicit cognitions. All predictors were mean-centered before entering into the models.

\section{RE S ULTS}

\section{Descriptives}

A total of 1790 individuals participated at baseline, out of which 314 were excluded as they either indicated to have been distracted during the SC-IAT or did not answer the control questions correctly. Hence a baseline sample of 1476 participants remained (47\% female, mean age $=49, \mathrm{SD}=15.90$ ). At the first follow-up after one month, 980 participants took part out of which 272 were excluded for the same reasons as mentioned above. Hence, the remaining sample at $\mathrm{T} 1$ consisted of 708 participants ( $48 \%$ of baseline, $47 \%$ female, mean age $=48, S D=15.18$ ). For the second follow-up, data of 556 participants were available out of which 89 were excluded. The remaining sample at T2 consisted of 467 data (32\% of baseline, $44 \%$ female, mean age $=50, S D=15.67$ ). At follow-up one, having a partner who eats $\mathrm{RM}$ meat predicted drop-out $(\mathrm{T} 1: \mathrm{OR}=2.95,95 \% \mathrm{Cl}[1.15,7.53], \mathrm{p}=.02)$. This variable was added in all analyses. No variable predicted drop-out at follow-up two. All characteristics of the sample as well as the differences of study variables over time are presented in table 4.1. Correlations and $95 \%$ confidence intervals of the study variables at baseline are presented in table 4.2. During the three waves, none of the participants reported to have ceased red-meat consumption. RMC was correlated with all measured study variables. Implicit attitudes were positively correlated with perceived pros and RMC and negatively correlated with perceived cons, self-efficacy, social norms, and all three intention items. Perceived pros and perceived cons were correlated to all other measured explicit cognitions. 
Table 4.1 | Characteristics of study sample and differences over time.

\begin{tabular}{|c|c|c|c|c|c|c|}
\hline & $\begin{array}{c}\text { T0 } \\
(\mathrm{N}=1476) \\
\mathrm{M}(\mathrm{SD})\end{array}$ & $\begin{array}{c}\text { T1 } \\
(\mathrm{n}=708) \\
\mathrm{M}(\mathrm{SD})\end{array}$ & $\begin{array}{c}\text { T2 } \\
(\mathrm{n}=467) \\
\mathrm{M}(\mathrm{SD})\end{array}$ & $\mathbf{F}$ & df & $\mathbf{p}$ \\
\hline Age & $49(15.90)^{*}$ & $48(15.18)$ & $50(15.67)^{\star \star}$ & 1.22 & 2 & .30 \\
\hline Gender (female), n (\%) & 692 (47\%) & 332 (47\%) & 178 (44\%) & - & - & - \\
\hline Perceived Pros & $3.50(.56)$ & $3.54(.59)$ & $3.56(.59)$ & 1.92 & 2 & .15 \\
\hline Perceived Cons & $2.06(.56)$ & $2.08(.56)$ & $2.12(.57)$ & 2.57 & 2 & .08 \\
\hline Social Norms & $2.53(.68)$ & $2.48(.70)$ & $2.49(.69)$ & 1.65 & 2 & .19 \\
\hline Social Modeling (partner, "Yes"), n (\%) & $992(67)$ & $475(32)$ & $329(22)$ & - & - & - \\
\hline Social Modeling (family members) & $4.35(.83)$ & $4.36(.79)$ & $4.29(.84)$ & 1.17 & 2 & .31 \\
\hline Social Modeling (friends) & $3.96(.70)$ & $3.99(.69)$ & $3.92(.71)$ & 1.64 & 2 & .20 \\
\hline Social Modeling (colleagues) & $3.53(.67)$ & $3.56(.65)$ & $3.51(.65)$ & 1.18 & 2 & .31 \\
\hline Self-efficacy & $3.17(.77)$ & $3.18(.77)$ & $3.15(.78)$ & .36 & 2 & .70 \\
\hline Intention & $2.24(1.93)$ & 2.30 (1.99) & $2.40(2.02)$ & 1.26 & 2 & .29 \\
\hline Intention (Likeliness to change) & $2.08(1.10)$ & $2.03(1.08)$ & $2.11(1.08)$ & .81 & 2 & .45 \\
\hline Intention (Strength) & $3.49(2.53)$ & $3.48(2.53)$ & $3.67(2.54)$ & 1.09 & 2 & .34 \\
\hline Implicit attitude & $-.03(.32)$ & $-.06(.32)$ & $-.05(.31)$ & 2.82 & 2 & .06 \\
\hline Red meat consumption (gr/week) & $473.50(435.77)$ & $493.06(388.34)$ & $484.23(344.78)$ & .57 & 2 & .57 \\
\hline
\end{tabular}

${ }^{*} \mathrm{n}=1461$, due to incomplete answers

${ }^{* *} n=401$, due to incomplete answers 


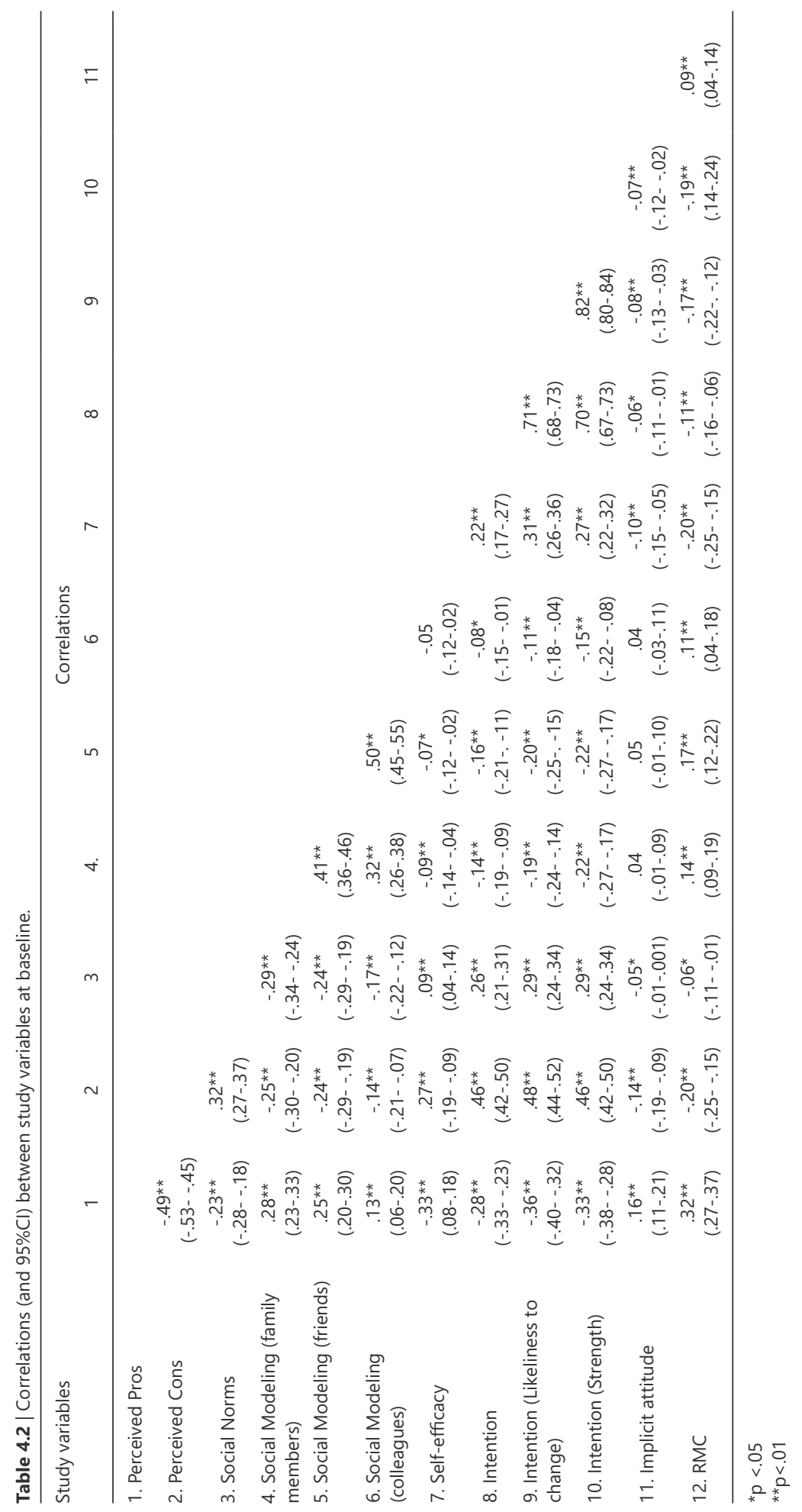


Research question 1

Do implicit attitudes and explicit cognitions predict (a reduced) red meat intake in addition to each other?

Implicit attitudes did not add directly to the prediction of RMC neither at baseline ( $F_{\text {change }}(1$, $588)=.11, \beta=.13, p=.74)$ nor after one month $\left(F_{\text {change }}(1,273)=.004, \beta=.004, p=.95\right)$ or after three months' follow-up $\left(F_{\text {change }}(1,161)=.53, \beta=-.05, p=.47\right)$. At baseline, perceived pros $(\beta$ $\left.=.20, \mathrm{p}<.001,95 \% \mathrm{Cl}^{1}[97.53,268.24]\right)$ and intention strength $(\beta=-.15, \mathrm{p}=.04,95 \% \mathrm{Cl}[-55.53$, -1.51]) were significant predictors for RMC, explaining $12 \%$ of variance.

After one month, perceived pros $(\beta=.28, p<.001,95 \% \mathrm{Cl}[120.91,329.25])$, norms $(\beta=$ $.12, p=.05,95 \% \mathrm{Cl}[.68,127.44])$ and intention to change $(\beta=.16, p=.05,95 \% \mathrm{Cl}[.56,72.81])$ explained $21 \%$ of variance in RMC and after three months, being male $(\beta=-.15, p=.04$, $95 \% \mathrm{Cl}[-193.94,-5.92])$, perceived pros $(\beta=.36, \mathrm{p}<.001,95 \% \mathrm{Cl}[116.77,312.81])$ and intention strength $(\beta=-.30, p=.04,95 \% \mathrm{Cl}[-82.09,-2.90])$ explained $31 \%$ of variance in RMC.

Research question 2

\section{Do implicit attitudes and explicit cognitions interact in the prediction of (a reduced) red meat consumption?}

At baseline, the interaction between implicit attitudes and self-efficacy showed a trend towards significance $(\beta=.08, p=.08,95 \% \mathrm{Cl}[-16.31,318.09])$. Follow-up stratified analyses demonstrated that the effect was strengthened significantly by negative implicit attitudes towards RM $(\beta=-.20, p=.006,95 \% \mathrm{Cl}[-142.95,-24.89])$ but not for neutral $(\beta=-.02, p$ $=.83,95 \% \mathrm{Cl}[-156.90,126.66])$ or positive implicit attitudes towards $\mathrm{RM}(\beta=.06, p=.42$, $95 \% \mathrm{Cl}[-41.08,98.51])$. This indicates that the effect of self-efficacy on RMC is strengthened when the person holds a negative implicit attitude towards red meat. Along with perceived pros and intention strength, the interaction between self-efficacy and implicit attitude added significantly to the prediction of $\operatorname{RMC}\left(\mathrm{F}_{\text {change }}(1,588)=4.93, \mathrm{p}=.03\right)$ and explained $13 \%$ of the variance.

After one and after three months, no significant interaction effects between implicit attitudes and explicit cognitions were detected.

\section{Research question 3}

\section{Do implicit attitudes and explicit cognitions interact in the prediction of the intention to reduce $R M C$ ?}

At baseline the interaction between social norms and implicit attitudes showed a trend towards significance regarding intention (item 1$)(\beta=.07, p=.07,95 \% \mathrm{Cl}[-.04,1.11])$. Stratified analyses demonstrated that this effect was significant when implicit attitudes were negative ( $\beta$ $=.15, \mathrm{p}=.03,95 \% \mathrm{Cl}[.03, .73])$ and positive $(\beta=.28, \mathrm{p}<.001,95 \% \mathrm{Cl}[.31, .92])$ but not when they were neutral $(\beta=.10, p=.15,95 \% \mathrm{Cl}[-.08, .54])$. This indicates that the effect of social

1 Unstandardized $95 \% \mathrm{Cls}$ are reported in the whole chapter. 
norms on RMC is strengthened when the individual holds a negative or a positive implicit attitude towards red meat. Regarding the items intention likeliness and intention strength, no significant interactions were found.

After one month' follow-up, interactions were non-significant. After three months, only the interaction between implicit attitudes and social norms regarding the intention likeliness to change was significant $(\beta=-.21, p=.03,95 \% \mathrm{Cl}[-1.31,-.08])$. Stratified analyses however did not reveal significant results (negative: $\beta=.10, p=.46,95 \% \mathrm{Cl}[-.20, .43]$; neutral: $\beta=.06, p=$ $.68,95 \% \mathrm{Cl}[-.35, .53]$; positive: $\beta=.02, \mathrm{p}=.89,95 \% \mathrm{Cl}[-.37, .43])$.

\section{Research question 4}

\section{Do implicit attitudes and explicit cognitions predict the intention to reduce red meat consumption in addition to each other?}

At baseline, no direct effects of implicit attitudes were detected regarding the intention to reduce $R M$ intake (item 1$)\left(F_{\text {change }}(1,591)=.05, \beta=-.01, p=.83\right)$, intention likeliness $\left(F_{\text {change }}\right.$ $(1,591)=.88, \beta=-.03, p=.35)$, and intention strength $\left(F_{\text {change }}(1,591)=.002, \beta=.002, p=\right.$ .96). Education $(\beta=.08, p=.03,95 \% \mathrm{Cl}[.02, .24])$, perceived cons $(\beta=.37, \mathrm{p}<.001,95 \% \mathrm{Cl}[.98$, $1.58])$, social norms $(\beta=.18, p<.001,95 \% \mathrm{Cl}[.24, .61])$, having a partner who does not eat red meat $(\beta=-.10, p=.007,95 \% \mathrm{Cl}[-1.65,-.27])$ and self-efficacy $(\beta=.12, p=.001,95 \% \mathrm{Cl}$ $[.14, .51])$ explained $30 \%$ of the intention (item 1$)$. Intention likeliness was explained by being female $(\beta=.10, p=.005,95 \% \mathrm{Cl}[.07, .38)]$, age $(\beta=.07, p=.05,95 \% \mathrm{Cl}[.00, .01])$, perceived $\operatorname{pros}(\beta=-.12, p=.004,95 \% \mathrm{Cl}[-.41,-.08])$, perceived cons $(\beta=.31, p<.001,95 \% \mathrm{Cl}[.44, .77])$, social norms $(\beta=.19, p<.001,95 \% \mathrm{Cl}[.16, .36])$, and self-efficacy $(\beta=.19, \mathrm{p}<.001,95 \% \mathrm{Cl}[.19$, .39]) which together explained $37 \%$ of the variance. Intention strength was predicted by being female $(\beta=.09, p=.02,95 \% \mathrm{Cl}[.07, .80])$, age $(\beta=.13, p=.001,95 \% \mathrm{Cl}[.01, .04])$, perceived cons $(\beta=.34, p<.001,95 \% \mathrm{Cl}[1.09,1.84])$, social norms $(\beta=.17, p<.001,95 \% \mathrm{Cl}[.28, .73])$, having a partner who does not eat red meat $(\beta=-.07, p=.05,95 \% \mathrm{Cl}[-1.73,-.02])$, the amount of friends eating RM $(\beta=-.10, p=.02,95 \% \mathrm{Cl}[-.70,-.05])$, and self-efficacy $(\beta=.14, p<.001$, $95 \% \mathrm{Cl}[.22, .68])$ which together explained $33 \%$ of variance.

After one month, implicit attitudes added directly to intention (item 1$)\left(F_{\text {change }}(1,276)\right.$ $=5.52, \beta=-.12, p=.02)$ and explained along with perceived cons $(\beta=.43, p<.001,95 \% \mathrm{Cl}$ $[1.09,1.98])$ and norms $(\beta=.19, p=.001,95 \% \mathrm{Cl}[.21, .76]) 29 \%$ of the variance. Also regarding the item intention likeliness, implicit attitudes added significantly to the prediction $\left(F_{\text {change }}(1\right.$, $276)=4.45, \beta=-.10, p=.04)$. Together with age $(\beta=.13, p=.02,95 \% \mathrm{Cl}[.002, .02])$ and the explicit cognitions perceived cons $(\beta=.47, p<.001,95 \% \mathrm{Cl}[.66,1.12])$ and norms $(\beta=.18, p=$ $.001,95 \% \mathrm{Cl}[.09, .38])$, they explained $36 \%$ of the variance. Intention strength was not directly explained by implicit attitudes $\left(F_{\text {change }}(1,276)=2.71, \beta=-.08, p=.10\right)$ but was predicted by age $(\beta=.12, p=.03,95 \% \mathrm{Cl}[.003, .04])$, perceived cons $(\beta=.49, p<.001,95 \% \mathrm{Cl}[1.58,2.61])$, and norms $(\beta=.16, p=.003,95 \% \mathrm{Cl}[.16, .80])$. All regression coefficients are depicted in table 4.3 . 
After three months, intention (item 1$)$ was not explained by implicit attitudes $\left(F_{\text {change }}(1,164)\right.$ $=.31, \beta=-.04, p=.58)$ but by perceived cons only $(\beta=.40, \mathrm{p}<.001,95 \% \mathrm{Cl}[.82,2.17])$. Also the items intention likeliness and intention strength were predicted by perceived cons only (intention likeliness: $\beta=.37, p<.001,95 \% \mathrm{Cl}[.33,1.02]$; intention strength: $\beta=.43, p<.001$, $95 \% \mathrm{Cl}[1.13,2.70])$ and not by implicit attitudes (intention likeliness: $F_{\text {change }}(1,164)=.41, \beta=$ $.05, p=.52$; intention strength: $\left.F_{\text {change }}(1,164)=.03, \beta=-.01, p=.87\right)$. 


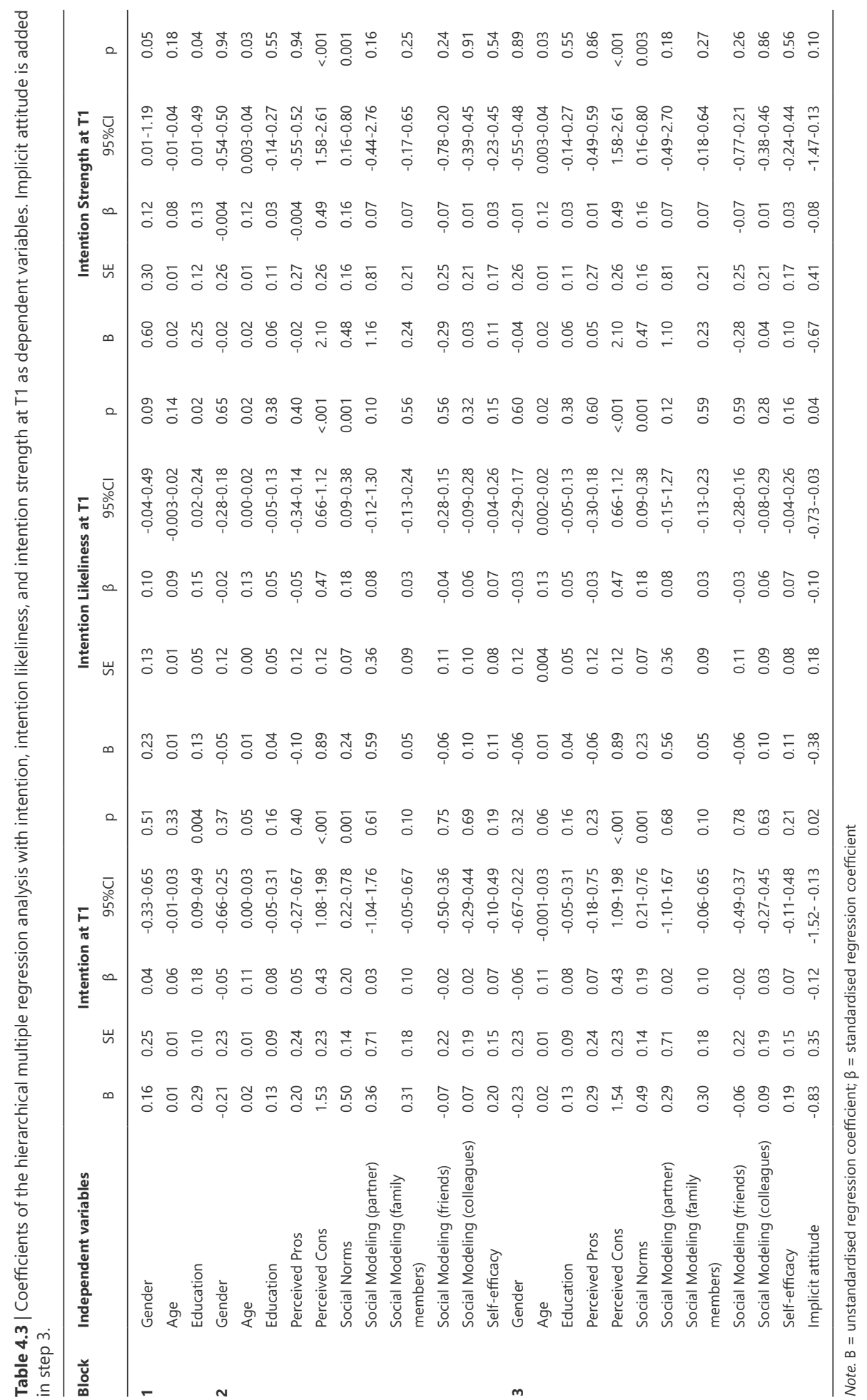




\section{DISCUSSION}

The study at hand provided insight into how implicit attitudes and explicit cognitions operate in the prediction of the intention to reduce RM intake as well as in the prediction of (a reduced) RMC. Additive as well as interaction patterns between these determinants were examined.

Implicit attitudes were found to be weakly positively correlated with RMC (at baseline), but were not associated with RMC after controlling for explicit cognitions neither at baseline nor at any later measuring point. Thereby, our results do not suggest support for the existence of the additive pattern for the prediction of RMC. To our knowledge, this is the first study to investigate the direct effect of both implicit attitudes and explicit cognitions on RMC. Only one similar study in the context of food choices has been conducted by Richetin et al. (2007) who found implicit attitudes to predict a person's snack and food choice besides explicit attitudes, thus evidence for the additive pattern. This pattern was not found for RMC allowing to conclude that the mode of operation between implicit and explicit determinants is not generalizable to one domain (e.g. eating behavior) but differs depending on the specific (eating) behavior. Another explanation for the different findings could be the different (methodological) approaches. Whereas Richetin et al. (2007) used the IAT and confirmed the additive pattern for the choice of snacks relative to the choice of fruits, we assessed the implicit attitude towards red meat unrelated to an opposed category. Further, we used a self-report measurement in order to assess RMC. Although this was based on the Food frequency scale - a widespread and accepted way of measuring food intake - self-reports are prone to reporting errors. Richetin et al. (2007) on the contrary used more direct assessments, i.e. participants had to choose a snack or a fruit after the experiment. Thus potential self-report bias was excluded. However, it needs to be stressed that the behavioural measure of Richetin et al. (2007) was conducted directly after the experiment, and therefore priming or social desirability effects cannot be ruled out. In order to draw a more generalizable conclusion about whether RMC is directly influenced by implicit attitudes or not, a follow-up study with a more objective measure of $\mathrm{RMC}$ is recommended.

Although the additive pattern could not be supported, support for the interactive pattern was found. At baseline, the relationship between self-efficacy and RMC was moderated by implicit attitudes and significantly strengthened by negative implicit attitudes. That is, people who consider themselves (explicitly) as capable of reducing their RM intake, show a lower RMC especially when they hold a negative implicit attitude towards RM. This appears logical and is in line with findings of a study from Muschalik et al. (2018) who also found implicit attitudes to moderate the relationship between self-efficacy and physical activity behavior. This finding supports the idea of an interactive pattern of influencing RMC and suggests that negative implicit attitudes are beneficial in order to foster the likelihood that self-efficacy decreases RM intake in the short-term.

Regarding the intention to reduce one's red meat intake, support for the additive as well as for the interactive pattern of operation was found. This is in line with our expectation 
that both ways of operation do not exclude each other. More precisely, baseline implicit attitudes significantly predicted the intention to change one's red meat intake as well as the intention likeliness after one month, with more positive implicit attitudes towards RM being associated with a lower intention. Although a few studies have considered direct effects of implicit processes on intention (Brochu \& Morrison, 2007; Dal Cin et al., 2007; Dasgupta \& Rivera, 2008), this is still a rather uncommon approach as the effect of implicit attitudes is mostly investigated on behavior. The findings of our studies, which are in line with former studies, indicate however that implicit (and explicit) attitudes are not exclusively associated with behavior but also with its most proximate determinant intention. Based on our findings and the findings of other authors, we suggest a reconsideration of the assumptions made in theoretical models, i.e. RIM (Strack \& Deutsch, 2004), which mostly assume only a direct effect of implicit attitudes on behavior but not on intention. Hence, adding intention in the RIM appears to be a logical extension.

Furthermore, not only a direct but also an indirect effect of implicit attitudes on intention was identified. That is, the interaction between social norms and implicit attitudes was significant for baseline intention to change. The effect of social norms on the intention to change was strengthened by positive as well as by negative implicit attitudes towards social norms, but not by neutral implicit attitudes. The finding that negative implicit attitudes strengthen the effect of social norms on intention appears logical, as it implies that other peoples' expectations to reduce one's RMC have a stronger increasing effect on intention when the person also has a negative implicit attitude towards RM. The very same relationship was also strengthened by positive implicit attitudes and seems surprising in the first place. However, Muschalik et al. (2018) found a similar pattern. In their study, the effect of social modeling on the intention to become physically active was significantly strengthened by negative implicit attitudes towards physical activity. The authors argued that resulting from the opposing cognitions, participants experienced dissonance and were motivated to resolve this dissonance that is normally associated with tensed or negative feelings. In order to do so, they assumingly denied the negative implicit attitude and acted in line with the (more accessible) behavior of others. It is plausible that also in the present study, the explicit knowledge that other people expect oneself to reduce one's RMC and the opposing unconscious preference for red meat created dissonance. In this case, people would also be motivated to resolve this dissonance and one way to do so could be by denying the less accessible positive implicit attitude and by acting in accordance with the more available social norms - i.e. indicating a high intention to change. Although this way of dissonance resolving has not been demonstrated in the context of eating behaviors, a similar technique was revealed in a study about smoking (Maliszewski, 2011) in which people with a negative implicit attitude and a positive explicit attitude towards smoking resolved this conflict by inhibiting their less accessible negative implicit attitude and by acting upon the more accessible positive explicit attitude, i.e. by smoking a cigarette. It is conceivable, that participants in the present study used a similar approach as it appears easier to follow the more obvious and accessible norms of others than the unconscious attitude. 
Although one could argue that positive implicit attitudes towards RM were beneficial in this context, one has to take into account that this was assumingly induced by dissonance. In case that social norms are low (e.g. an individual does not experience other people to expect him or her to reduce RM intake), a positive implicit attitude towards RM would possibly strengthen this effect and lower the individual's intention.

Based on our findings, it can be said that next to tackling explicit cognitions, health interventions that are aiming at an intake below 300gr/week could benefit from training or changing implicit attitudes regarding RM towards a negative direction. Until now, this is not a common approach as interventions to reduce meat consumption mostly address explicit cognitions, e.g. by means of self-monitoring (Carfora et al., 2017a; Carfora, Caso, \& Conner, 2017b). Although these studies led to a lower meat intake, one could argue that changing implicit attitudes in addition to that might yield results that are even more successful as these were also related to reduced RM intake and intention in our study. An attempt to alter food related implicit attitudes into a negative direction has been undertaken by Hollands, Prestwich and Marteau (2011). They paired images of energy-dense snack foods with aversive images of the potential health consequences of unhealthy eating and found baseline positive implicit attitudes towards energy-dense snacks to be more negative after the experiment. Although this approach has not been applied to the consumption of RM, pairing pictures of red meat with aversive images in a computerized task appears to be a feasible way to complement interventions that already aim at altering explicit cognitions towards red meat consumption. Additionally, our results suggest that the role and place of implicit associations may not always be completely distinct as suggested by the RIM (Strack \& Deutsch, 2004). Social cognitive models, such as the Reasoned Action Approach (Fishbein \& Ajzen, 2011) or the I-change model (De Vries, 2017), need to address the importance of implicit associations and research is needed to identify how to best depict the pathways of both implicit and explicit factors.

\section{Limitations}

When interpreting our findings, the following possible limitations need to be taken into account. First, as mentioned above, we assessed participants' RMC by means of self-reports which is prone to reporting errors. In order to confirm that the additive pattern cannot be applied to RMC, a follow-up study with a measure of RM intake that is less sensitive to bias would be valuable (e.g. a food diary, pictures of consumed food). Furthermore, the intention constructs were entered separately into the analyses. Although the use of single-item scales is a widespread and accepted method to assess (dietary related) intention (Ajzen \& Fishbein, 1980; Grønhøj, Bech-Larsen, Chan, \& Tsang, 2012; Øygard \& Rise, 1996; Patch, Tapsell, \& Williams, 2005; Rezai, Teng, Mohamed, \& Shamsudin, 2012), constructs measured by several items or a full scale are agreed on to be more reliable (Lowenthal, 2001; Nunnally \& Bernstein, 1994). Therefore, considering a follow-up study with multi-item measures of the intention constructs would be worthwhile. In addition, Hofman and colleagues (2005) argued that the relation between implicit and explicit attitudes can be influenced by design factors, such as the 
correspondence of measurements. We assessed explicit cognitions (including explicit attitude) regarding red meat consumption and implicit attitudes towards red meat, as implicit attitudes towards red meat consumption appeared to be rather difficult to be assessed via the SC-IAT, i.e. stimuli that clearly represents the consumption of red meat. This is a general problem of the SC-IAT. Many other studies used a similar approach, e.g. assessing implicit attitudes towards smoking by using smoking related stimuli (e.g. words/pictures of cigarettes, tobacco, nicotine, ashtray etc.) to predict smoking behavior (Huijding, de Jong, Wiers, \& Verkooijen, 2005; Waters et al., 2007). However, as a consequence, the correspondence between the measurements might have been reduced. Not only follow-up studies should take this into account, but more research regarding the design of the SC-IAT is needed in order to find a possible solution for this issue.

\section{CONCLUSION}

This research has taken an important first step to illustrate that both explicit cognitions and implicit attitudes are associated with the intention to reduce ones' RM intake as well as with RMC and demonstrated that negative implicit attitudes towards red meat are more beneficial when aiming at a higher intention and a lower RM intake. Future research should seek to replicate the findings of additive and interactive patterns and should also examine whether tackling both implicit attitudes and explicit cognitions does indeed result in more significant decreases of RM intake than when tackling one type of cognition only. Shedding light on these questions may help to achieve a transition to a less meat-based diet and could thereby improve peoples' health as well as the health of the planet. 

Chapter 


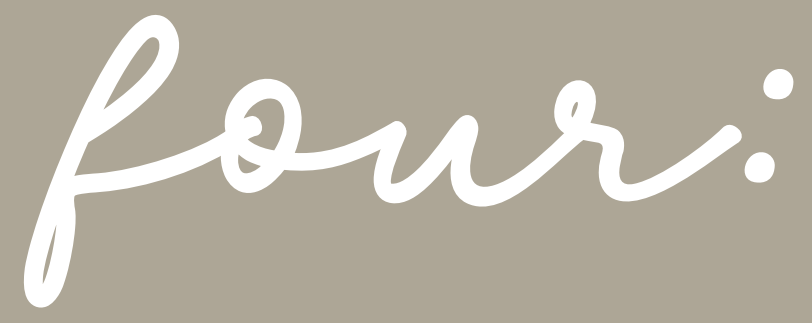

\section{Mindfulness is not associated with dissonant attitudes but enhances the ability to cope with them}

Muschalik, C., Crutzen, R., Candel, M. J.J., Elfeddali, I. \& de Vries, H. (in press). 


\section{A B S T R A C T}

Background: Explicit and implicit attitudes have been studied extensively, but there is less attention to reducing dissonance between them. This is relevant because this dissonance (IED) results in distress and has inconsistent effects on behavior, e.g. less physical activity but more smoking. Mindfulness decreases dissonance between self-related explicit and implicit constructs. This study investigates if, and which, specific mindfulness subskills are associated with decreased dissonance between explicit and implicit attitudes, and whether mindfulness subskills moderate the relationship between IED and intention/behavior.

Method: At baseline and one and three months thereafter, participants' $(N=1476)$ explicit attitudes, implicit attitudes, red meat consumption (RMC), intention to reduce $\mathrm{RMC}$ as well as levels of trait mindfulness were assessed.

Results: Mindfulness subskills were not associated with decreased IED. IED was associated with lower RMC and a higher intention to reduce RMC. The mindfulness subskill acceptance buffered the effect of IED on intention, seemingly offering a skill to deal with dissonant attitudes, which was unidentified until now.

Conclusion: The mindfulness subskill accepting without judgment functions as a way to deal with dissonance. Future research should use this novel finding and investigate whether mindfulness can be used as a buffer in contexts where dissonance results in maladaptive behaviors. 


\section{INTRODUCTION}

Dual-process models suggest that individuals have two sources for their evaluative tendencies (Gawronski \& Bodenhausen, 2007; Hofmann et al., 2008; Sheeran et al., 2013; Strack \& Deutsch, 2004; Wilson et al., 2000). The first source roots in intentional reasoning and is based on beliefs, which the individual considers as true. These beliefs are presented in explicit constructs towards an object or behavior (e.g. an explicit attitude). The second source draws on intuitive feelings and automatic evaluations towards a target and shapes a person's implicit tendencies (e.g. an implicit attitude or an approach or avoidance tendency). These rather unconscious evaluations occur regardless of whether the individual considers them as true or not (Gawronski \& Bodenhausen, 2006). According to dual-process models, explicit constructs are part of the reflective system. Evaluations within this system are traditionally assessed through self-reports. Automatic evaluations, on the contrary, are part of the impulsive system. These evaluations are inferred by reaction time tasks, such as the Implicit Association Task (IAT; Greenwald et al., 1998). It is assumed that both types of evaluations can occur simultaneously (Fazio \& Olson, 2003; Perugini, 2005; Wilson et al., 2000) and that they can be in line with each other (i.e. the explicit and implicit evaluations regarding a subject are both positive or both negative), but also dissonant (i.e. one evaluation is positive whereas the other is negative).

Dissonance between these two types of evaluations has mainly been explored for selfrelated topics such as self-esteem or affective experiences (Brown \& Ryan, 2003; Koole et al., 2009; Zeigler-Hill, 2006). This dissonance has been shown to result in psychological distress and negative health outcomes, such as unhealthy forms of perfectionism, higher levels of narcissism, being more vulnerable to criticism, being more prone for anger suppression (Bosson, Brown, Zeigler-Hill, \& Swann, 2003; Schröder-Abé, Rudolph, \& Schütz, 2007; Vater, Schröder-Abé, Schütz, Lammers, \& Roepke, 2010; Zeigler-Hill, 2006) as well as in diminished physical and psychological health (Schröder-Abé et al., 2007). Hence, congruence between implicit and explicit evaluations might decrease distress and improve health outcomes.

One factor that has been shown to enhance congruence between dissonant implicit and explicit evaluations is mindfulness (Brown \& Ryan, 2003; Koole et al., 2009). Mindfulness can be understood as "the awareness that emerges through paying attention on purpose, in the present moment, and nonjudgmentally to the unfolding of experience moment by moment" (Kabat-Zinn, 2003, p. 145). Mindfulness can be divided into five different facets or subskills: observing, describing, acting with awareness, accept without judgment, and non-reactivity to inner experiences (Baer, Smith, Hopkins, Krietemeyer, \& Toney, 2006; Raes, Dewulf, Van Heeringen, \& Williams, 2009). Observing is the ability to observe, notice, and attend to internal and external phenomena (e.g. bodily sensations, or smells). Describing refers to the ability to describe, label, or note observed phenomena by using words in a non-judgmental way. Acting with awareness concerns the ability to be attentive and fully engaged in one's present activity, and accepting without judgment is the tendency to accept one's thoughts and feelings without evaluating them. Non-reactivity to inner experiences is the ability to allow one's thoughts and 
feelings to come and go, without getting carried away by them or acting on them. In sum, mindfulness involves the pure perception of one's internal and external experiences in an objective way. Since this mindful processing enhances the clarity of one's thoughts, feelings, behaviors, and sensations (Brown, Ryan, \& Creswell, 2007), it may allow intuitive, implicitly represented information about objects (i.e., implicit attitudes) to become incorporated into a person's conscious experience (i.e., explicit attitude). In addition, mindfulness facilitates the ability to observe one's (inner) experiences without judgment or attempts to change those. Hence, mindful individuals may also be more acceptive of their implicit attitudes once they become aware of them. We do not assume that implicit attitudes are more 'correct' than explicit attitudes, but that mindfulness might help to get in touch with a person's implicit attitude. This might result in expressing one's implicit attitude also in an explicit way. Thereby, discrepancy between a person's implicit and explicit attitude might be decreased.

Also Brown and Ryan (2003) concluded that mindfulness can facilitate the uncovering of rather inaccessible (unconscious or automatic) realities. Participants in their study that scored higher on dispositional (trait) mindfulness had a greater congruence between their implicitly and explicitly measured affective experience (i.e. the current experience of positive and negative emotions). Koole et al. (2009) revealed that meditation - a practice that aims to promote mindfulness - enhanced the congruence between implicitly and explicitly measured self-esteem. Presumably, mindfulness increased the sensitivity and attention towards inner (rather unconscious) emotions, which are normally less accessible. This insight, in turn, was used to reflect on one's explicit report and resulted in a more accurate evaluation of self-related constructs, which then resulted in more congruent attitudes. Until now, a few studies have investigated in how far mindfulness enables individuals to decrease the level of dissonance regarding self-related constructs, which are normally highly emotionally laden. Self-esteem, for example, is defined as a "favourable or unfavourable attitude toward the self" (Rosenberg, 1965). Hence, the self is in the focus of the evaluation. It is unclear, whether mindfulness can also help to decrease the level of dissonance between implicit and explicit attitudes constructs that are, although also to some degree, less self-relevant and less experiential, but more actionable. An attitude is defined as "a psychological tendency that is expressed by evaluating a particular entity with some degree of favor or disfavor" (Eagly \& Chaiken, 1993, p. 1 ). Hence, not the self is the focus of the evaluation but another entity. To our knowledge, the question whether mindfulness can decrease dissonance between implicit and explicit attitudes regarding entities other than the self has been unaddressed and builds, therefore, the focus of this study.

As far as we know, only Hyde and colleagues (2010) performed a similar study. More precisely, they investigated whether the congruence between implicit and explicit attitudes regarding physical activity is moderated by private self-consciousness and private body consciousness. No effect was found. Private self-consciousness is defined as the tendency to focus on internal thoughts and feelings (Fenigstein, Scheier, \& Buss, 1975), and private body consciousness as the ability to be sensitive to internal bodily states (Miller, Murphy, 
\& Buss, 1981). These definitions correspond with the mindfulness subskill observing, which can be defined as the ability to observe, notice or attend to stimuli including internal phenomena (cognitions, bodily sensations) and external phenomena (sounds, smells) (Baer, Smith, \& Allen, 2004). However, it is reasonable to assume that in order to translate one's implicit attitude into one's explicit attitude, it is not only necessary to be able to have insight into one's inner processes (i.e. observing or private body consciousness) but also to accept one's inner processes as they are (i.e. accepting without judgement). For example, when they are inconsistent with one's personal belief system or perceived social norms. Additionally, for the translation of implicit attitudes into explicit statements, it might be essential that an individual does not react automatically in case dissonance between his or her implicit and explicit attitudes is detected. For example, by trying to alter his or her inner experiences (i.e. non-reactivity to inner experiences). The exclusive focus on private self-consciousness and private body consciousness (i.e. observing) might be a reason for the null findings in the study of Hyde et al. (2010). Moreover, neither of the aforementioned studies (Brown \& Ryan, 2003; Koole et al., 2009) investigated which specific mindfulness subskill(s) lead to a greater congruence between implicitly and explicitly measured constructs, but simply concluded that the ability to have insight into one's inner processes (i.e. observing) was responsible for that. Consequently, the present study investigated not only whether trait mindfulness is related to the congruence between implicit attitude and explicit attitudes, but also which subskill of trait mindfulness is responsible for this occurrence. This was done in an exploratory way.

Attitudes are a key construct in social psychology and are regarded as important determinants across various (health) behaviors. Both implicit and explicit attitudes can predict the same (health) behavior (Conroy et al., 2010; Friese et al., 2007) and individuals can hold dissonant explicit and implicit attitudes towards the same behavior or object (Karpen et al., 2012; Petty et al., 2006; Rydell et al., 2008; Rydell et al., 2006). Similarly to dissonant selfrelevant constructs, dissonant attitudes are experienced as unpleasant (Rydell et al., 2008) and lead to difficulties in functioning, which individuals are highly motivated to diminish. More importantly, this dissonance has an impact on (health) behaviors (Goldstein et al., 2014; Maliszewski, 2011; Muschalik, Elfeddali, Candel, Crutzen, \& de Vries, 2019; Rydell et al., 2008). Participants with more dissonant implicit and explicit attitudes towards a person were more motivated to engage in additional information processing regarding that person (Rydell et al., 2008), which was presumably an attempt to decrease dissonance. Also, more dissonant implicit and explicit attitudes towards physical activity are associated with lower levels of physical activity (Muschalik et al., 2019). Individuals are assumingly uncertain about whether to move towards the behavior or not. On the other hand, smokers with dissonant implicit and explicit attitudes towards smoking are more likely to resolve this conflict by smoking a cigarette (Maliszewski, 2011) and more dissonant implicit and explicit attitudes result in more disinhibited chocolate consumption (Goldstein et al., 2014). The authors explained that the dissonance intensified the focus on the object (chocolate), which in turn resulted in higher consumption. Moreover Goldstein et al. (2014) found the personality trait impulsivity 
to moderate the effect between the dissonance of attitudes and behavior, in the sense that dissonance was predictive for individuals with high levels of impulsivity but not for individuals with low levels of impulsivity.

The aforementioned studies demonstrate that the effects of implicit-explicit discrepancy (IED) on behavior are inconsistent (i.e. more information processing, less physical activity, more smoking, more disinhibited chocolate consumption). Therefore, the question arises whether the effect of IED on behavior could be moderated by a third variable. Goldstein et al. (2014) demonstrated that IED predicted disinhibited eating especially in individuals high in levels of impulsivity. According to Murphy and MacKillop (2012) "impulsivity and mindfulness are natural reciprocals" (page 528). Although both constructs entail a focus on the present moment, the present moment is approached differently. That is, impulsivity entails an overemphasis on the present moment, without an adequate reflection on the future or on consequences of one's action (Patton, Stanford, \& Barratt, 1995). Mindfulness also entails a focus on the present moment, however, by noticing and experiencing it fully. Hence, it occurs without judgment and reactivity. Therefore, impulsivity reflects a greater likelihood to act on an impulse, and mindfulness reflects a decreased likelihood to do so, which stems from the acknowledgment of impermanence (i.e. everything comes and goes) (e.g. Marlatt, 2002). It has been demonstrated that mindfulness enables individuals to refrain from maladaptive impulsive behaviors (Peters, Erisman, Upton, Baer, \& Roemer, 2011). Therefore, we conducted exploratory analyses to investigate whether the effect of IED on behavior is moderated by mindfulness skills.

In sum, the aim of the study is twofold. First, we investigated whether mindfulness is related to more congruent implicit and explicit attitudes and, if so, we explored which subskills are associated with that (RQ 1). Second, we assessed whether the mindfulness subskills moderate the relationship between IED and behavior (RQ 2a) and additionally, we explored whether the mindfulness subskills moderate the relationship between IED and intention (RQ 2b). According to the Reasoned-Action Approach (Fishbein \& Ajzen, 2011) or the Integrated-Change Model (De Vries, 2017), intention is the most proximate antecedent for behavior. It is unclear, whether IED affects behavior only or also its most proximate antecedent and whether this relationship might be moderated by the mindfulness subskills.

\section{METHOD}

\section{Design}

The current study is a follow-up study of a larger study, in which different predictive models of implicit attitudes and explicit cognitions regarding (the intention to reduce) red meat consumption (RMC) were tested. The study protocol of the original study was preregistered at https://osf.io/7enj9/?view_only=d1afaf26fdbe4f13a9feb0d857c89db0. IED and mindfulness were not part of the previous study. In the study at hand, emphasis lies on the relationships 
between mindfulness, IED, behavior, and intention. Shedding light on these questions in the context of RMC could support the development of future interventions that are aiming to reduce RMC, which has been defined as a threat for people's health (e.g. Pan et al., 2011) and the environment (Aiking, 2014; Westhoek et al., 2014).

\section{Ethical approval}

The FHMLRec, the ethical committee of a Dutch University, provided ethical approval for this study (Muschalik/220517).

\section{Power analysis}

To determine the sample size, we conducted a power analysis by means of G*Power. Small effect sizes were anticipated for main and interaction effects ( $f 2=0.03$ ) and the test power was set at 0.80 with a type I error rate of $\alpha=0.05$ for two-sided testing. The calculation revealed that a minimum sample of 488 is required. Based on former experiences of the internet research agencies that were collaborated within this study, a drop-out of $60 \%$ between the baseline and the second follow-up (T2) was expected. Therefore, the aim was to have data of 1220 participants available at the first measurement (after having implemented various exclusions), in order to have data of $\mathbf{4 8 8}$ participants available at the second follow-up.

\section{Procedure}

We conducted a three-wave longitudinal study with a baseline measurement (T0), a followup after one month (T1) and another follow-up after three months (T2). The whole study was conducted online among a sample of the Dutch population (47\% female, age range: $18-89$ years at baseline), which was recruited among members of two independent Internet panels that operate in line with the ISO standards (Flycatcher Internet Research, 2018; Research Now, 2018). All individuals who were older than 18 years and had previously indicated to consume meat regularly, were invited by email to participate. When willing to participate, information was provided explaining that the study aims to gain insight into the relationship between determinants related to eating behavior and that there would be three measurements, that one measurement would take 15-20 minutes to complete, that each measurement was comprised of a reaction time task and a questionnaire, that participation was free of risks, that all data would be gathered and analyzed anonymously, and that they would receive a monetary reward. To begin with the study, participants needed to read and agree upon an electronic informed consent. If participants did not do so, they were excluded from further participation. At the beginning of the study, we included a question to double-check whether only people who consumed red meat at least once a month participated. People who answered the question with "no" were excluded. One and three months after baseline, a new invitation was sent to those participants who had participated previously to complete the follow-up. Depending on the standards for payment used in the two different Internet panels, participants received $€ 2.50$ or $€ 4.75$ for participation in the baseline, $€ 2.50$ or $€ 3.00$ for participation in $T 1$, and $€ 4.00$ or $€ 5.00$ for participation in T2. 


\section{Measurements}

Implicit attitude assessment task. The Single-Category Implicit Association Test (SC-IAT; Karpinski \& Steinman, 2006) was used to assess participants' implicit attitudes towards red meat. In earlier studies, the SC-IAT has demonstrated satisfactory internal consistency (Karpinski \& Steinman, 2006). Opposed to the IAT, the SC-IAT does not require a contrasting concept (e.g. man vs. women) but measures the implicit attitude unrelated to a contrast category. As we were interested in the implicit attitudes towards red meat unrelated to a contrast category, the SC-IAT was selected. Positive and negative words from the Affective Norms for English Words (ANEW; Bradley \& Lang, 1999) were used as evaluative stimuli. They were translated forth and back from English to Dutch by Dutch native researchers of Maastricht University. Subsequently, the Dutch words were pretested regarding their perceived levels of valence (1 = 'very negative' to $9=$ 'very positive'), arousal ( $1=$ 'not arousing at all' to 9 = 'very arousing'), and familiarity ( 1 = 'very unfamiliar' to $9=$ 'very familiar') by 28 people. Words with the highest scores regarding positivity and familiarity and similar arousal levels were selected as positive stimuli (love, friend, freedom, humor, joy; translated from Dutch). Words with the lowest scores on positivity, highest scores of familiarity and similar evaluations of arousal were selected as negative stimuli (death, hate, devil, loneliness, lie; translated from Dutch). To represent red meat, pictures were derived from the study of De Houwer and De Bruycker (2007) and from the Internet (i.e., Creative Commons Images). These were pretested by the same sample regarding their representativeness for red meat ( 1 = 'not representative at all', 2 = 'not so strongly/a bit representative', 3 = 'strongly representative'). Seven pictures that were evaluated as to be the most representative, were included in the SC-IAT.

We programmed the SC-IAT by means of the software Inquisit by Millisecond (Version 4) with the script being based on Karpinski and Steinman (2006). The SC-IAT is comprised of two blocks which each consisted of 24 practice trials and 72 test trials. In one block "red meat or negative" versus "positive" are the two categories, in the reversed block "red meat or positive" versus "negative" built the two categories. One after another, negative or positive words or pictures of red meat were presented in the middle of the screen and participants were instructed to indicate as quickly as possible to which of the two categories the stimulus belonged. The two blocks were presented in a counterbalanced order. The assumption that underlies the SC-IAT is that when an individual is quicker with categorizing the stimuli when "red meat or negative" are one category than when "red meat or positive" are one, the individual's implicit attitude regarding red meat is negative and vice versa. Throughout the whole task, labels of the categories were displayed on the right and left upper part of the computer screen. When a stimulus belonged to the category that was shown on the right upper part of the screen, participants had to press $i$ on their keyboard. When the stimulus belonged to the category displayed on the left upper part of the screen, they had to press $e$. All stimuli were presented equally frequent and randomized. If an answer was incorrect, a red $X$ appeared on the screen until it was corrected. 
The implicit attitude was represented by $d$-scores, which were calculated by the Inquisit software. The $d$-score represents the strength of an association between concepts, which is measured by the standardized mean difference score of the 'hypothesis-inconsistent' pairings and 'hypothesis-consistent' pairings and is expressed in milliseconds (Greenwald et al., 2003). More positive $d$-scores indicate a more positive reaction to red meat. Normally, $d$-scores range from -2 to 2 and all participants in our sample had a d-score within this range. After they had performed the SC-IAT, participants were asked whether they were distracted during the task. Different types of distraction were offered (e.g. 'I was talking on the phone', 'I was eating or drinking', 'I was listening to music' etc.). Only when participants selected the option 'I was not distracted', their d-score was included in the analyses. Based on this control question, data of 185 participants (13\%) were excluded afterward. The internal reliability of the SC-IAT was calculated by dividing the SC-IAT into thirds (blocks of 24 test trials). For each third, a SC-IAT score was calculated (Karpinski \& Steinman, 2006) and the average intercorrelation among these three scores was identified by means of the Spearman-Brown formula, which is conceptually equivalent and comparable to Cronbach's alpha in terms of range (i.e., from 0 to 1 , where higher values indicate stronger internal consistency). The internal consistency was deemed acceptable $(r=.73)$. The test-retest correlations between implicit attitudes at baseline and T1 ( $r=.18, p<.01)$, implicit attitude at baseline and T2 ( $r=.09, p<.05)$, and implicit attitude at T1 and T2 $(r=.21, p<.01)$ were significant, but fall within the lower range of test-retest reliabilities. However, these values are comparable to published studies using implicit measures such as a race-attitude Implicit Association Tests (0.17-0.50) (Cunningham, Preacher, \& Banaji, 2001), aggressiveness Implicit Association Tests (0.14-0.39) (Gollwitzer, Banse, Eisenbach, \& Naumann, 2007) or political Single Target-Implicit Association Tests (0.210.46) (Bluemke \& Friese, 2008).

Subsequently, participants filled in a questionnaire, which is described in the following. The SC-IAT was performed first, as a prior assessment of explicit cognitions is assumed to trigger red meat-related thoughts which would in turn influence the reaction time in a following task (Bargh \& Chartrand, 2000).

Self-report assessment. The I-change model (De Vries, 2017; De Vries et al., 2005) has previously been used to identify eating-related cognitions (Schulz et al., 2014; Walthouwer et al., 2015) and was used in the present study to assess explicit attitudes towards RMC and intention to reduce RMC. The questionnaire can be found at https://osf.io/bkp5r/?view only $=6 c 2 e 208 b 9 e 8 f 4354 a c 339 c 2596 b 85 c 2 f$.

Explicit attitude was assessed with the two scales perceived pros and perceived cons regarding RMC. Each scale is comprised of 10 statements on a 5-point Likert Scale, which were based on beliefs underlying the attitudes towards meat (Dibb \& Fitzpatrick, 2014; Verbeke \& Viaene, 1999). An example of a pro statement is "Eating red meat is" (1) "very nutritious" to (5) "not nutritious". As two items showed a low factor loading, they were removed from the scale $(\Omega=.73)$. Items were reversed so that higher values represent the perception of more pros. An example of a con statement is "Eating red meat is" (1) "very disgusting" to (5) "not disgusting". 
Due to a low factor loading one item was removed $(\Omega=.66)$. For the analysis, a sum score for the pro scale and a sum score for the con scale was created. Both scales were added to represent one scale for explicit attitude (range -40 to 40 ) that was used in the analyses. The higher the score, the more positive the explicit attitude.

Intention to reduce RMC was measured by three different items. The first item asked "Are you planning to eat less red meat in the future?" with answer options ranging from (1) "No, I am not planning to reduce my red meat intake" to (7) "Yes, within one month". The second item asked "The chance that I will eat less red meat within the next three months is" (1) "very unlikely" to (5) "very likely". The third item (intention strength) asked participants to indicate on how strongly he/she was planning to reduce his/her red meat intake within the next three months with a scale from (1) "very little" to (10) "very strongly". Factor saturation of the standardized sum scores was estimated as insufficient $(\Omega=.07)$, therefore intention items were entered separately in the analyses as. Higher scores on all items represent a stronger intention to reduce RMC.

To assess mindfulness, a Dutch translation of the KIMS-E (Baer et al., 2006; Raes et al., 2009) was administered. In contrast to earlier studies that suggested mindfulness to be a unidimensional construct (Brown \& Ryan, 2004; MacKillop \& Anderson, 2007), a more recent factor analysis regarding various mindfulness questionnaires identified five different domains of trait mindfulness (Baer et al., 2006). Given that the original KIMS (Baer et al., 2004) only consists of four facets, we decided to use the KIMS-E, which entails the fifth facet. Also, the KIMS-E has demonstrated good psychometric properties (Raes et al., 2009). The KIMS-E is a 46-item scale which entails the mindfulness subskills observing, describing, acting with awareness, and accept without judgment as well as the additional subskill non-reactivity to inner experience derived from the Five-Factor Mindfulness Questionnaire (Baer et al., 2006). All items were rated on a 5-point Likert scale ranging from (1) "never or very rarely true" to (5) "always or almost always true". Where appropriate, items were reversed so that higher scores indicate higher levels of mindfulness.

Observing was assessed by means of 12 items. An example is "I notice changes in my body, such as whether my breathing slows down or speeds up." Due to low factor loadings, two items were removed. A mean scale score of all remaining items was included in the analyses $(\Omega=.66)$.

Describing was measured by eight items, such as "Even when I'm feeling terribly upset, I can find a way to put it into words." We included a mean scale score for describing $(\Omega=.80)$ in the analyses.

Acting with awareness was administered by ten items. An example is "When I'm doing something, I'm only focused on what I'm doing, nothing else." Due to low factor loadings, four items were removed and a mean scale score was created out of the remaining items and included in the analyses $(\Omega=.56)$.

Accept without judgment was comprised of nine items. Example items are "I criticize myself for having irrational or inappropriate emotions" or, "I think some of my emotions are bad 
or inappropriate and I shouldn't feel them.". One item showed a low factor loading and was removed. All other items were combined to a mean scale score, which was included in the analyses $(\Omega=.83)$.

Nonreactivity to inner experience was assessed by seven items. Example items are "Usually when I have distressing thoughts or images, I just notice them and let them go" or "I perceive my feelings and emotions, without having to react to them". Due to low factor loadings, three items were removed and a mean scale score of the remaining items was included in the analyses $(\Omega=.70)$. As mindfulness has been shown to be a multidimensional and not a unidimensional construct (Baer et al., 2006) and since we were interested in the specific subskill(s) that might be associated with attitudinal dissonance, the sub-skills were entered as separate constructs in the analyses.

Red meat consumption was measured by means of the question "On how many days per week do you usually consume red meat" (ranging from 1-7 days per week and the additional answer option 'Not on a daily basis but at least once a month') and the open question "On days when you eat red meat, how many grams of red meat do you eat on average per day?" A reference point was provided, e.g. that a piece of prepared meat at the main meal equals $100 \mathrm{gr}$ and a slice of meat topping (e.g. ham) equals $15 \mathrm{gr}$. By multiplying the frequency by the number of grams, the weekly RMC was calculated. This procedure was based on the Food Frequency Questionnaire (FFQ) and former diet-related studies (Springvloet et al., 2016; Van Assema et al., 2002).

Two control questions were formulated (e.g. 'From the following answer options, please select statement $4^{\prime}$ ) to excluded data of those participants who did not answer the questionnaire thoroughly (e.g., straightlining or not paying attention).

\section{Analyses}

Scale quality of the measurements used in the present study was assessed by means of exploratory factor analyses as well as McDonald's omega (reported in the measurement section above) as a less biased alternative to Cronbach's alpha (Crutzen \& Peters, 2017). Omega $_{\text {hierarchical }}$ was used as an indicator of internal structure, which estimates factor saturation based upon the sum of the squared loadings of items on the general factor (McDonald, 2013). Logistic regressions were used to investigate whether dropout at $\mathrm{T} 1$ and $\mathrm{T} 2$ was predicted by the variables age, gender, educational level, explicit attitude, implicit attitude, IED, intention, $\mathrm{RMC}$, and the mindfulness subskills. To assess whether the measured variables differed over time, we conducted analyses of variance (ANOVAs).

Based on previous studies on IED (Brinol et al., 2006; Petty et al., 2007; Rydell et al., 2008), an index was created to evaluate the effects of IED. Therefore, we calculated the absolute value of the difference between the average of a participant's standardized explicit attitude score and the standardized reaction times of the SC-IAT. This index indicates where participants fall within the distribution of the sample on the implicit versus explicit versus measure, thereby representing the size of the discrepancy. When a person's place in the distribution is the same 
on the implicit and explicit measure (e.g. high in the distribution on both measures, low in the distribution on both measures, and so on), the index shows a value close to zero. The more the two attitudes deviate from each other (e.g. high in the distribution of explicit attitudes and low in the distribution of implicit attitudes or vice versa), the higher the score on the index and the further away it is from zero.

To assess possible cross-sectional and longitudinal associations between IED and the mindfulness subskills (RQ 1), we conducted correlational analyses between the baseline mindfulness subskills and IED at baseline, after one month, and after three months.

To assess a possible moderating effect of the mindfulness subskills on the relationship between IED and RMC (RQ 2a), three regressions were conducted. For short-term effects, we regressed participant's RMC levels at TO on TO IED and the mindfulness subskills observing, describing, acting with awareness, accepting without judgment, and nonreactivity in step one, and added the interactions between IED and all five mindfulness subskills in a second step. To assess long-term effects, the same regressions were repeated but with RMC at T1 and T2 as the dependent variable.

To investigate the additional question regarding intention and possible short-term and long-term effects of the mindfulness subskills on the relationship between IED and intention (RQ 2b), we conducted three regressions each with intention at baseline, at T1, and at T2 as the dependent variable. Baseline variables were again added in two steps of a regression. IED and the mindfulness subskills in step one, and the interactions between IED and the mindfulness subskills in a second step. In case significant interaction terms were found, followup stratified analyses were conducted (Aiken et al., 1991). In this case, the respective subskill was categorized into low, moderate, and high based on one standard deviation below, on, and above the mean.

To control for multiple testing, we used the Benjamini-Hochberg (Benjamini \& Hochberg, 1995; Benjamini \& Yekutieli, 2001) linear step-up method for the regression models. This method is considered more powerful and less conservative than the Bonferroni procedure (Benjamini \& Hochberg, 1995). By means of an Excel template, the adjusted p-values were calculated (Weinkauf, 2012). The Benjamini-Hochberg method ranks variables according to their $p$-values in increasing order. The smallest value gets rank 1 , the second rank 2 , and the largest value receives rank $\mathrm{N}$. Then, each $\mathrm{p}$-value is multiplied by $\mathrm{N}$ and divided by its assigned rank to give the adjusted Benjamini-Hochberg $\mathrm{p}$-value. For all regressions and stratified analyses, Benjamini-Hochberg $\mathrm{p}$-values are reported. 


\section{RE S U LTS}

At baseline, 1790 individuals participated. Participants who indicated that they were distracted during the SC-IAT or did not answer the control questions correctly were removed. Thereby a sample of 1476 remained $(47 \%$ female, mean age $=49, S D=15.90)$. After one month, data of 980 participants were available, out of which 272 were excluded for the same reasons as mentioned above. Thus, the sample at T1 consisted of 708 participants ( $48 \%$ of baseline, $47 \%$ female, mean age $=48$, SD $=15.18$ ). At T2, data of 556 participants were available out of which 89 were excluded. The remaining sample at T2 consisted of 467 data (32\% of baseline, $44 \%$ female, mean age $=50, S D=15.67$ ). Drop-out at T1 was significantly predicted by the mindfulness subskill acceptance without judgment $(\mathrm{OR}=1.34,95 \% \mathrm{Cl}[1.13,1.58], \mathrm{p}<.001)$ indicating that a higher acceptance was associated with a higher likeliness to drop-out. This variable was added to all analyses. Drop-out at T2 was not predicted by any of the measured variables. The implicit attitude towards red meat of the sample was slightly negative $(M=-.03$, $\mathrm{SD}=.32)$ and the explicit attitude was slightly positive $(M=9.53, S D=8.25)$. Implicit and explicit attitude were significantly positively correlated with each other $(r=.17)$. IED was present and moderately distinct $(\mathrm{M}=1.01, \mathrm{SD}=.80$, range $.00-5.51)$ and negatively correlated with implicit and explicit attitude $(r=-.06, r=-.18)$. The distribution of IED at baseline is presented in figure 5.1. Descriptives of all study variables and changes over time are displayed in table 5.1. The mindfulness subskills describing, acting with awareness, and acceptance without judgment changed significantly over time (i.e. decreased over time).

Table $\mathbf{5 . 1}$ | Descriptives and differences of study variables over time.

\begin{tabular}{|c|c|c|c|c|c|c|}
\hline & $\begin{array}{c}\text { T0 } \\
(\mathrm{N}=1476)\end{array}$ & $\begin{array}{c}\text { T1 } \\
(\mathrm{N}=708)\end{array}$ & $\begin{array}{c}\text { T2 } \\
(\mathrm{N}=467)\end{array}$ & $\mathbf{F}$ & df & $\mathbf{p}$ \\
\hline & $\mathrm{M}(\mathrm{SD})$ & $\mathrm{M}(\mathrm{SD})$ & $\mathrm{M}(\mathrm{SD})$ & $\mathrm{F}$ & df & \\
\hline Explicit attitude & $9.53(8.25)$ & $9.57(8.36)$ & $9.35(8.57)$ & .10 & 2 & .90 \\
\hline Implicit attitude & $-.03(.32)$ & $-.06(.32)$ & $-.05(.31)$ & 2.83 & 2 & .06 \\
\hline IED & $1.01(.80)$ & $1.03(.79)$ & $1.05(.76)$ & .68 & 2 & .50 \\
\hline Observing & $3.26(.58)$ & $3.21(.62)$ & $3.24(.63)$ & 1.22 & 2 & .30 \\
\hline Describing & $3.58(.65)$ & $3.01(.33)$ & $3.00(.35)$ & 376.36 & 2 & $<.001$ \\
\hline Acting with awareness & $3.21(.59)$ & $2.87(.49)$ & $2.87(.48)$ & 123.94 & 2 & $<.001$ \\
\hline Accepting without judgment & $3.76(.71)$ & $2.15(.74)$ & $2.20(.74)$ & 1567.98 & 2 & $<.001$ \\
\hline Non-reactivity & $3.06(.67)$ & $3.01(.67)$ & $3.13(.66)$ & 1.61 & 2 & .20 \\
\hline Intention (item 1) & $2.24(1.93)$ & $2.30(1.99)$ & $2.40(2.02)$ & 1.26 & 2 & .29 \\
\hline Intention (item 2) & $2.08(1.10)$ & $2.03(1.08)$ & $2.11(1.08)$ & .81 & 2 & .45 \\
\hline Intention (item 3) & $3.49(2.53)$ & $3.48(2.53)$ & $3.67(2.54)$ & 1.09 & 2 & .34 \\
\hline Red meat consumption (gr/week) & $473.50(435.77)$ & $493.06(388.34)$ & $484.23(344.78)$ & .57 & 2 & .57 \\
\hline
\end{tabular}




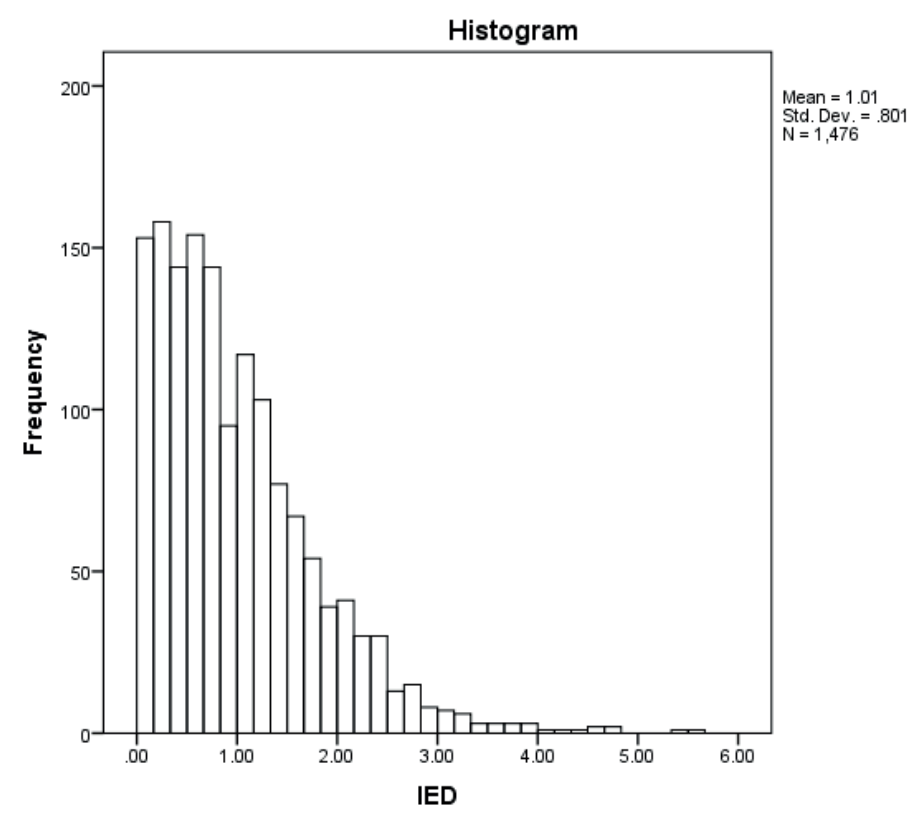

Figure.5.1. | Distribution of IED at baseline

Is mindfulness associated with congruent implicit and explicit attitudes? Correlations between study variables at baseline as well as correlations between IED at T1 and T2 with mindfulness subskills are depicted in table 5.2. Contrary to our expectation, none of the mindfulness subskills were associated with IED at baseline nor with IED after one or three months.

Do the mindfulness subskills moderate the relationship between IED and RMC?

\section{RMC at baseline}

For RMC at baseline, no significant interactions between IED and the mindfulness subskills were found. This indicates that, contrary to our expectation, mindfulness subskills do not moderate the relationship between IED and RMC. Tests for multicollinearity indicated a very low level of multicollinearity between the mindfulness subskills (VIF = 1.33 for observing, 1.36 for describing, 1.21 for acting with awareness, 1.30 for acceptance without judgment, and 1.15 for nonreactivity). Thus, nonsignificant findings are not due to multicollinearity. Lower RMC at baseline was significantly associated with higher IED $(B=-49.09, p=.004,95 \% \mathrm{Cl}[-76.69$, -21.49]), a higher distinction of the subskill observing $(B=-63.45, p=.004,95 \% \mathrm{Cl}[-106.91$, $-19.99])$, and a lower distinction of the subskills acting with awareness $(B=32.77, p=.23$, $95 \% \mathrm{Cl}[-7.79,73.32])$ and nonreactivity $(\mathrm{B}=31.31, \mathrm{p}=.22,95 \% \mathrm{Cl}[-4.00,66.62])$ (see table 5.3 for all regression coefficients). 


\section{RMC after one month}

For RMC after one month, no significant interactions between IED and the mindfulness subskills were found. Again, multicollinearity was very low (VIF = 1.32 for observing, 1.41 for describing, 1.24 for acting with awareness, 1.27 for acceptance without judgment, and 1.17 for nonreactivity) and can be excluded as a possible reason for nonsignificant findings. Lower RMC after one month was associated with higher IED ( $\mathrm{B}=-44.23, \mathrm{p}=.05,95 \% \mathrm{Cl}[-79.77$, -8.69]), a more distinct observing subskill $(B=-77.61, p=.008,95 \% \mathrm{Cl}[-131.57,-23.65])$, and with a lower distinct subskill acting with awareness $(B=50.85, p=.19,95 \% \mathrm{Cl}[-1.58,103.27])$ (see table 5.3 for all regression coefficients).

\section{RMC after three months}

Also for RMC after three months, no significant interactions between IED and the mindfulness subskills were found. Multicollinearity was again very low (VIF = 1.35 for observing, 1.52 for describing, 1.29 for acting with awareness, 1.44 for acceptance without judgment, and 1.17 for nonreactivity) and can, therefore, be ruled out as possible reason for nonsignificant findings. After three months, lower RMC was associated with higher IED $(B=-38.34, p=.09,95 \% \mathrm{Cl}$ $[-75.51,-1.16])$ and with a more distinct observing subskill $(B=-79.90, p=.09,95 \% \mathrm{Cl}[-143.32$, -16.48]) (see table 5.3 for all regression coefficients). 


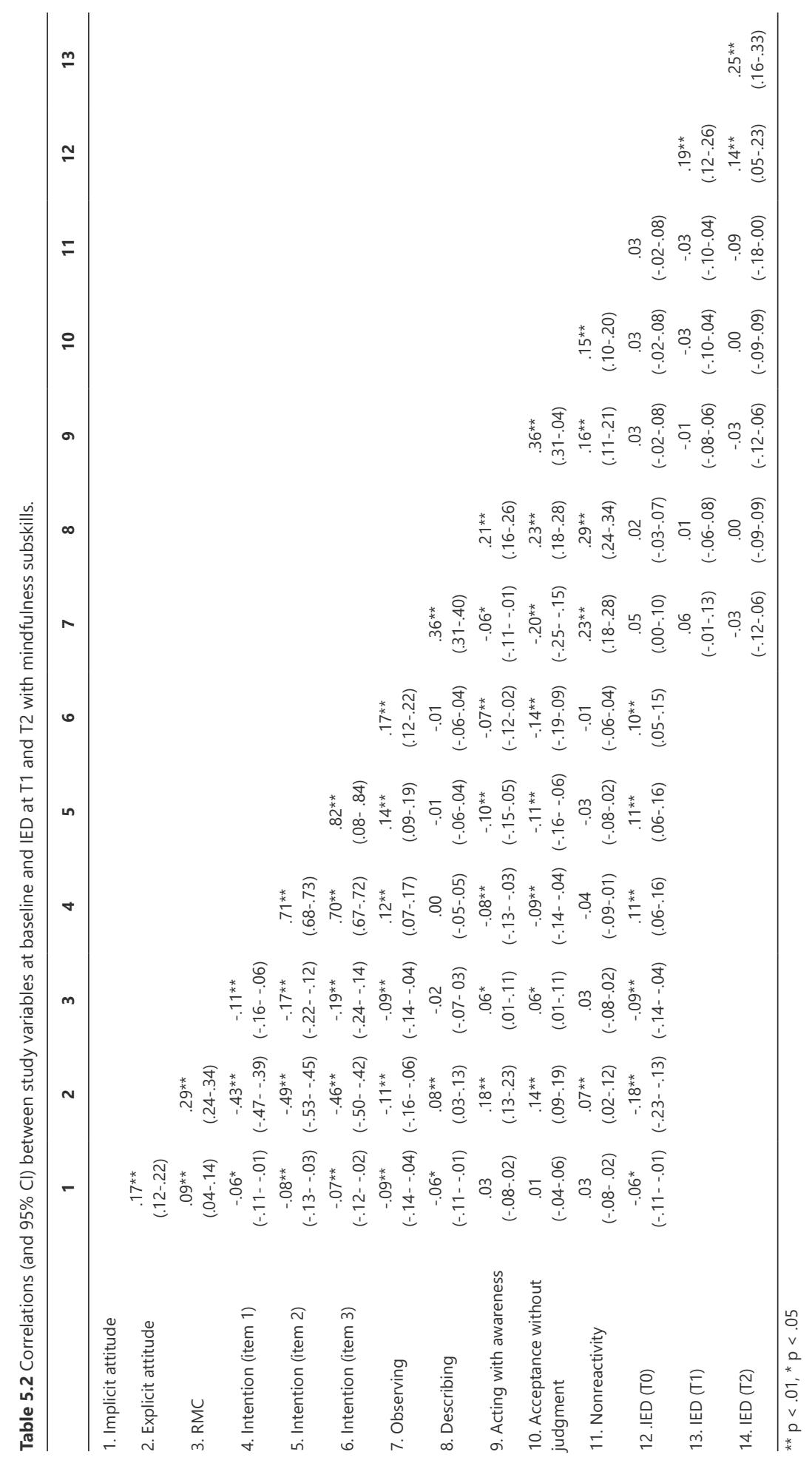




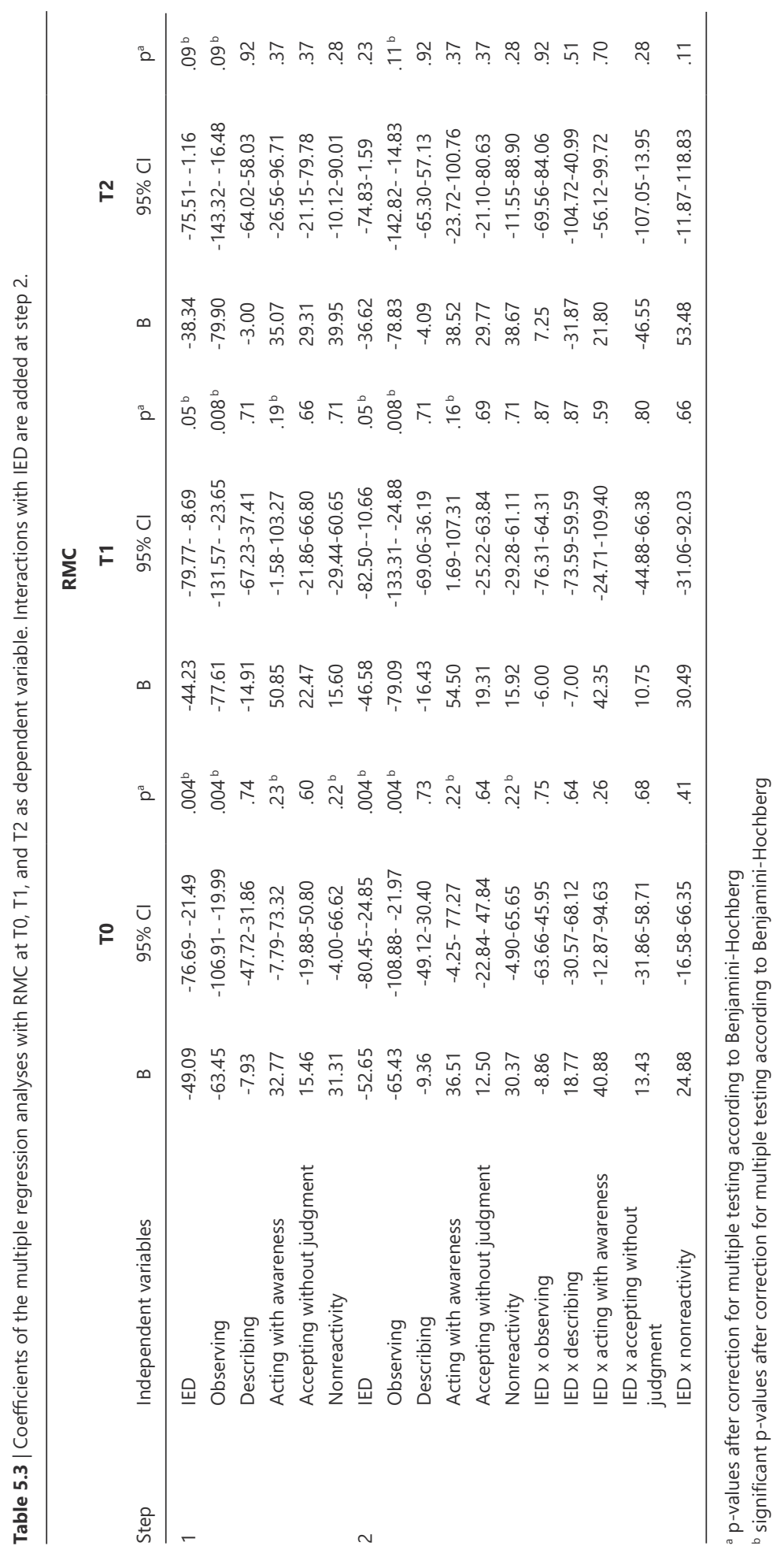




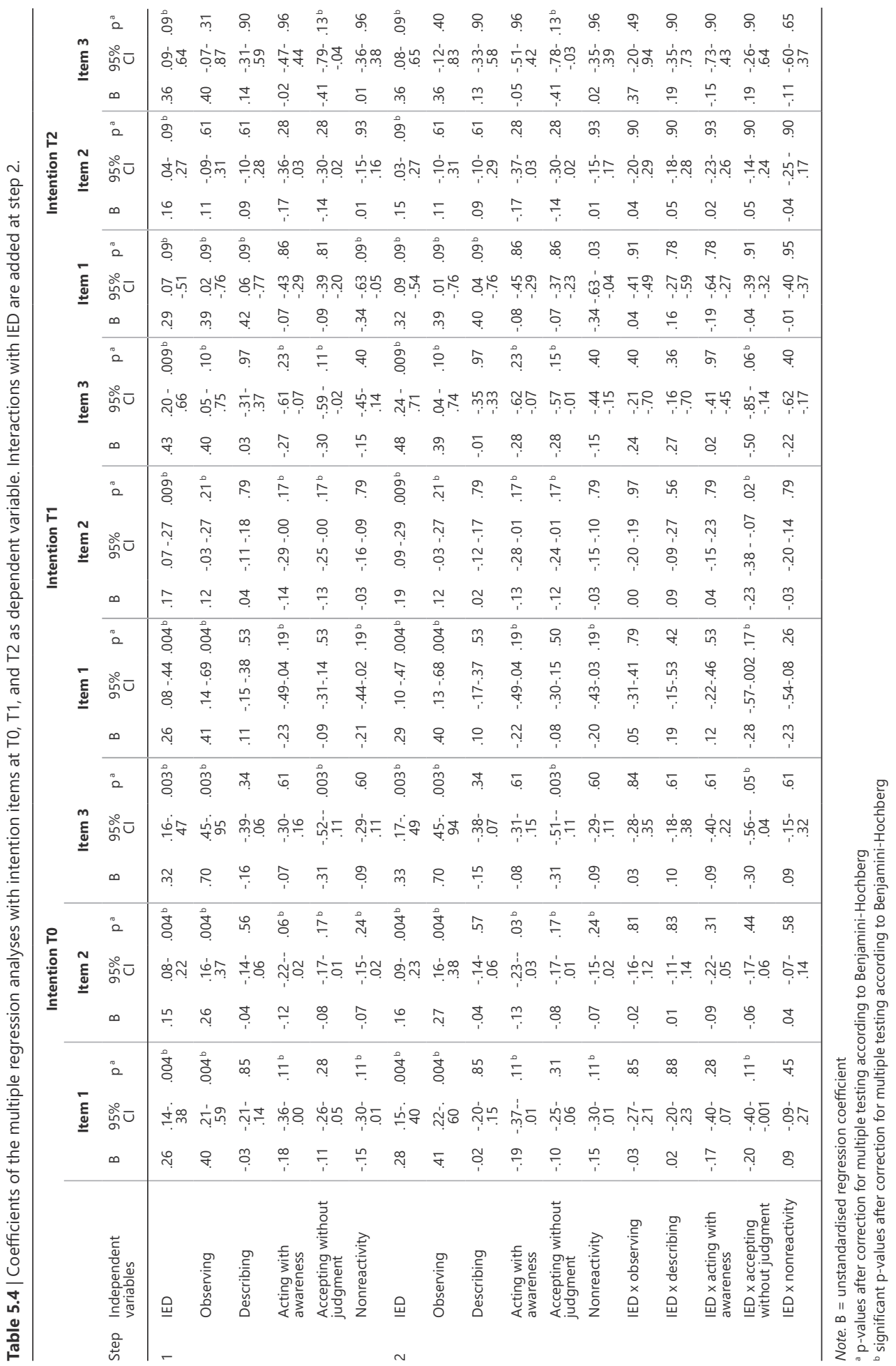


Do the mindfulness subskills moderate the relationship between IED and the intention to reduce RMC?

\section{Intention at baseline}

For the intention items 1 and 3 at baseline, a significant interaction between IED and accepting without judgment was found (item $1: \mathrm{B}=-.20, \mathrm{p}=.11,95 \% \mathrm{Cl}[-.40,-.001]$; item $3: \mathrm{B}=-.30, \mathrm{p}$ $=.05,95 \% \mathrm{Cl}[-.56,-.04]$ ) (see table 5.4 ).

Stratified analyses for item 1 revealed that IED had a positive effect on the intention to reduce $\mathrm{RMC}$ when accepting without judgment was low $(\mathrm{B}=.43, \mathrm{p}=.002,95 \% \mathrm{Cl}[.26, .61])$ and moderate $(B=.28, p=.002,95 \% C l[.16, .40])$, but not when it was high $(B=.12, p=.14$, $95 \% \mathrm{Cl}[-.04, .28])$ (see Figure $5.1 \mathrm{a}$ ).

Stratified analyses for item 3 revealed similar results. IED had a positive effect on the intention to reduce RMC when the ability to accept without judgment was low $(B=.54, p=$ $.002,95 \% \mathrm{Cl}[.31, .77])$ or moderate $(\mathrm{B}=.34, \mathrm{p}=.002,95 \% \mathrm{Cl}[.18, .50])$, but not when it was high $(\mathrm{B}=.13, \mathrm{p}=.22,95 \% \mathrm{Cl}[-.08, .34])$ (see Figure $5.1 \mathrm{~b})$.

For intention item 2, no significant interaction was found. In all three regressions, multicollinearity between the mindfulness subskills was very low (VIFs < 1.42). Hence, nonsignificant findings for this item are not due to multicollinearity.

\section{Intention after one month}

For all three intention items after one month, the interaction between IED and accepting without judgment was significant (item $1: \mathrm{B}=-.28, \mathrm{p}=.17,95 \% \mathrm{Cl}[-.57, .002]$; item 2: $\mathrm{B}=-.23$, $\mathrm{p}=.02,95 \% \mathrm{Cl}[-.38,-.07]$; item $3: \mathrm{B}=-.50, \mathrm{p}=.06,95 \% \mathrm{Cl}[-.85,-.14])$ (see table 5.4 ).

Stratified analyses for item 1 showed that IED had a positive effect on the intention to reduce $\mathrm{RMC}$ when accepting without judgment was low $(\mathrm{B}=.44, \mathrm{p}=.003,95 \% \mathrm{Cl}[.19, .70])$ and moderate $(B=.26, p=.009,95 \% C l[.07, .44])$, but not when it was high $(B=.07, p=.60$, $95 \% \mathrm{Cl}[-.19, .32])$ (Figure 5.2a).

For item 2, stratified analyses revealed also that IED had a positive effect on the intention to reduce $R M C$ when the ability to accept without judgment was low $(B=.31, p=.002,95 \% \mathrm{Cl}$ $[.17, .45])$ and moderate $(B=.16, p=.002,95 \% C l[.07, .26])$, but not when it was high $(B=.02$, $\mathrm{p}=.79,95 \% \mathrm{Cl}[-.12, .16])$ (Figure $5.2 \mathrm{~b}$ ).

The same pattern was revealed for item 3. IED had a positive effect on the intention to reduce $\mathrm{RMC}$ when acceptance without judgment was low $(B=.80, p=.002,95 \% \mathrm{Cl}[.48,1.12])$ and moderate $(B=.43, p=.002,95 \% C l[.20, .66])$, but not when it was high $(B=.05, p$ $=.76,95 \% \mathrm{Cl}[-.27, .37]$ ) (Figure $5.2 \mathrm{c}$ ). In all three regressions, multicollinearity between the mindfulness subskills was very low (VIFs $<1.55)$. 


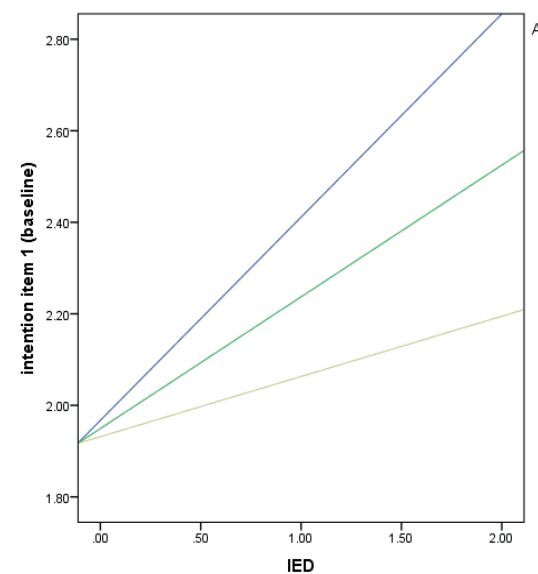

Figure 5.1a | Stratified analyses for the relationship between intention (item 1) at baseline and IED and the moderator acceptance without judgment.

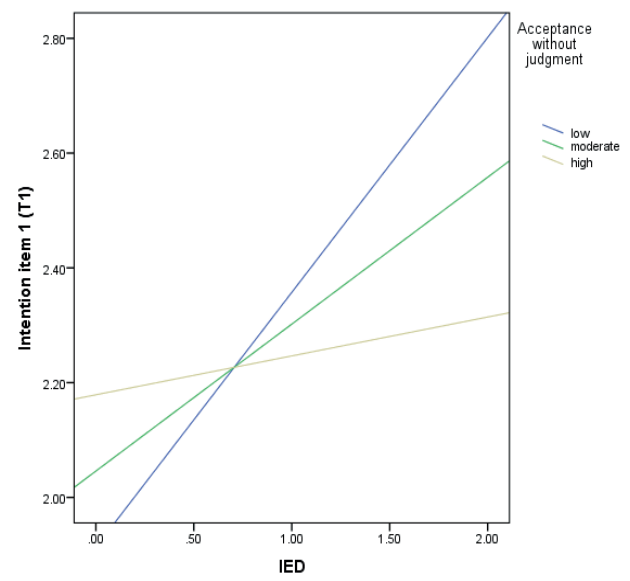

Fig. 5.2a | Stratified analyses for the relationship between intention (item 1) at T1 and IED and the moderator acceptance without judgment.

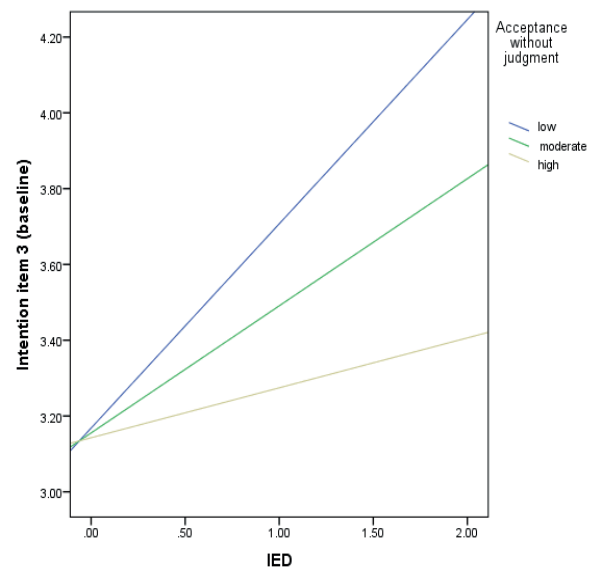

Figure 5.1b | Stratified analyses for the relationship between intention (item 3) at baseline and IED and the moderator acceptance without judgment.

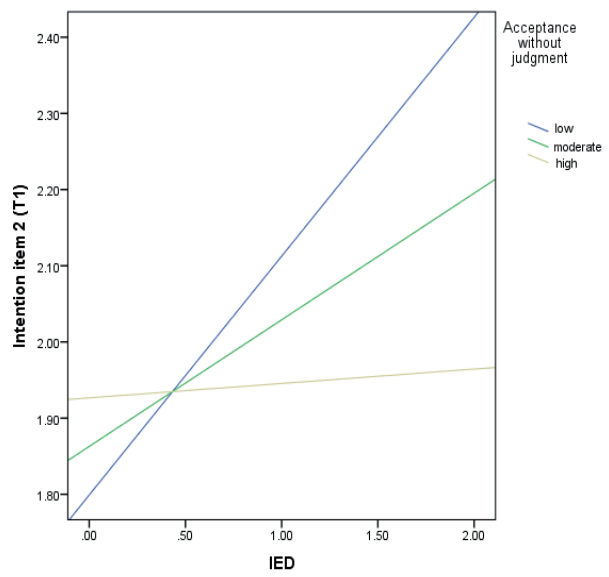

Fig. 5.2b | Stratified analyses for the relationship between intention (item 2) at T1 and IED and the moderator acceptance without judgment. 


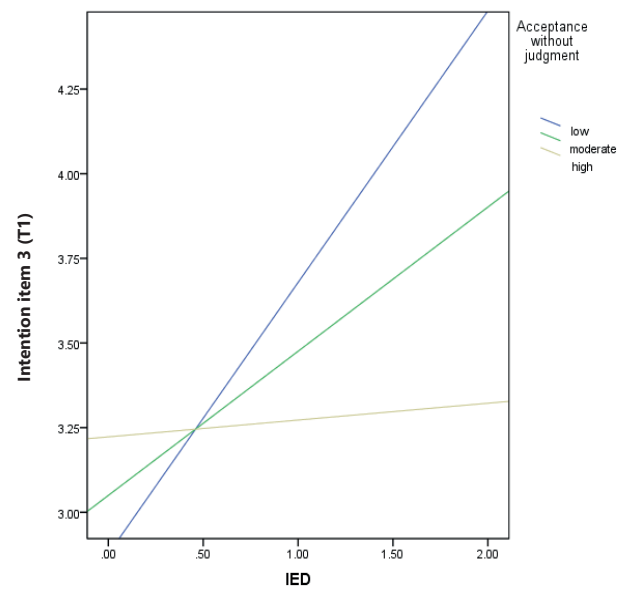

Fig. 5.2c $\mid$ Stratified analyses for the relationship between intention (item 3) at T1 and IED and the moderator acceptance without judgment.

\section{Intention after three months}

For intention items after three months, no significant interactions between IED and the mindfulness subskills were found. All regression coefficients are depicted in table 5.4. Multicollinearity between the mindfulness subskills was very low in all three regressions (VIFs $<1.65$ ) and can therefore be ruled out as possible reason for nonsignificant findings.

\section{DISCUSSION}

We did not find any mindfulness subskill to be associated with the level of congruence between implicit and explicit attitudes, which is contrary to our expectation. This finding is, however, partly in line with the findings of Hyde et al. (2010) and Hofmann et al. (2005), which also did not show an association between private self-consciousness and the congruence between implicit and explicit attitudes towards physical activity or different ethnicities, respectively. Both studies used a measurement comparable to the mindfulness subskill observing. Therefore, we anticipated that not only the ability to observe inner processes but also other processes, such as the ability to accept one's inner processes as they are or non-reactance to it, are required to translate them into explicit statements. This would then ultimately lead to more congruent attitudes. However, this was not the case in the study at hand. It is possible that mindfulness is completely unrelated to the level of congruence between implicit and explicit attitudes. Yet, studies on self-esteem (Brown \& Ryan, 2003; Koole et al., 2009) or the need for achievement (Thrash et al., 2007) demonstrated that mindfulness or private body consciousness decreased dissonance between the implicitly and explicitly measured constructs. It is possible that this effect only applies to highly self-relevant and emotionally charged constructs and not to 
rather cognitively based constructs, such as attitudes. In order to draw more generalizable conclusions, it would be worthwhile to investigate (a) whether the present findings do or do not apply to IED in other less self-relevant domains and (b) whether mindfulness or its subskills have an effect on the congruence between implicit attitudes and only affective explicit attitudes, which are more emotionally charged.

We did not find any mindfulness subskill to moderate the relationship between IED and RMC. These null-findings could be due to different reasons. Firstly, RMC was assessed by means of self-reports. It is possible that reporting errors lead to bias in terms of the amount of consumption, thereby also leading to a distorted illustration of the relationship between IED, $\mathrm{RMC}$, and the moderating role of the mindfulness subskills. A second reason could be that the mindfulness subskills simply do not moderate the relationship between IED and RMC (i.e., lack of a true effect). Whether this is the case should however be investigated by future research making use of a more objective measure of consumption. Furthermore, it would be worthwhile investigating whether these findings also apply to other types of behavior. Thereby it could be investigated whether other (health) behaviors are also positively or negatively affected by IED and whether mindfulness could function as an "antidote" in case the effect is negative.

An additional finding of the present study was that IED was related to behavior at all three measurement points, which was also the case in former studies (Brinol et al., 2006; Goldstein et al., 2014; Maliszewski, 2011; Muschalik et al., 2019; Petty et al., 2006; Rydell et al., 2008). In the current study, the impact was negative. Hence, individuals with dissonant implicit and explicit attitudes regarding RMC consume less red meat. This can be explained by Festinger's cognitive dissonance theory (Festinger, 1957), which postulates that individuals strive for congruence between their attitudes and behaviors. When inconsistency occurs, individuals are motivated to resolve it, as it elicits an aversive state of arousal. To do so, there are different ways such as justifying one's behavior or cognition (e.g. "I am allowed to eat red meat once in a while"), by adding new cognitions (e.g. "Other people also eat red meat"), ignoring or denying information that conflict with existing beliefs (e.g. "There is no problem with eating red meat") or by changing one's behavior (e.g. "I will not eat red meat anymore"). From the present results it could be concluded that individuals mainly used the last strategy, namely behavior change, which is in line with the assumption of Festinger (1957) that behavior change is the most preferred one. Furthermore, these findings are in line with the outcomes of a former review which concluded that dissonance results in behavior change (Stone \& Fernandez, 2008).

Our results regarding the relationship between IED and intention suggest that there exists an additional way to deal with dissonance, which has, to our knowledge, not been identified before in the literature. The mindfulness subskill acceptance without judgment moderated the relationship between IED and intention. For people with a low and only moderate ability to accept their thoughts and feelings without judging them (including possible negative feelings resulting from IED), higher IED increased the intention to reduce RMC. For people with a strong ability to accept their inner processes as they are, IED did not affect intention, hence no reaction was triggered by IED. This finding fits nicely with the assumption that mindful 
people are better able to control their reactions as they have a greater acceptance of their own 'errors' and associated conflicts (Hajcak \& Foti, 2008) as well as with the idea that by feeling their affective signals more keenly, more mindful individuals are in a better position to react to them, including an adequate control of their behavior or reaction (Teper, Segal, \& Inzlicht, 2013). Findings of a study of Haddock et al. (2017) confirmed these ideas as more mindful people reported to feel more comfortable with holding ambivalent or dissonant views. This effect was shown for dissonance between explicit beliefs as well as between one's implicit and explicit sexual orientation. Hence, it can be concluded that although mindfulness does not lead to more congruent attitudes, it seems to offer a skill to deal with dissonant attitudes, namely the 'simple' acceptance of it. This effect was not present anymore after three months and could be due to the smaller sample size at that time point.

In the present study, the non-acceptance of dissonance had a positive and thereby desirable effect on the intention to reduce RMC. Future studies should investigate in which contexts dissonance results in healthy behaviors, as it was the case in the present study, and in which contexts dissonance results in rather unhealthy behaviors, as demonstrated in former studies (Goldstein et al., 2014; Maliszewski, 2011; Muschalik et al., 2019). Shedding light on these questions would allow interventions to consciously induce dissonance in order to achieve certain behavior changes. However, in the context where dissonance has been shown to be maladaptive, e.g. self-esteem (Maliszewski, 2011; Vater et al., 2010), future research needs to investigate whether acceptance can be used as an alternative strategy to deal with dissonance. Attempts to alter attitudes would thereby become superfluous. Therapy approaches, such as Acceptance and Commitment Therapy (Hayes, Luoma, Bond, Masuda, \& Lillis, 2006), already entail this approach. Regarding attitude dissonance resolution, an interesting avenue for future research would be to compare the effectiveness of attitude retraining with acceptance-based approaches. Another possible avenue for future studies could be the investigation of self-compassion in the context of discrepancies. Self-compassion is an even broader construct than mindfulness as it includes the components of mindfulness, self-kindness, and common humanity. It is defined as a kind and understanding outlook toward one's personal disappointments and struggles (Neff, 2003a; Neff, 2003b). A study on body appreciation concluded that self-compassion might work as a buffer against harshly judging discrepancies regarding body-related comparisons. Whether self-compassion might also prevent a judgmental view against attitudinal discrepancies could be investigated in the future.

Several possible limitations of this study should be taken into account. First, RMC was measured by self-report. Although based on the Food Frequency Questionnaire, a validated tool also for the assessment of meat intake (Steinemann et al., 2017), self-reports have been defined as less reliable as they are more prone to reporting errors than objective measurements. It is possible that the null-findings regarding our second hypothesis were a result of the usage of a self-report, which did not depict the 'true' relationship between IED and RMC. To conclude whether the relationship between IED and RMC is not moderated by levels of mindfulness, we encourage studies to include a more objective measurement of meat-eating behavior. 
A second possible limitation could be that the SC-IAT measured implicit attitudes towards red meat whereas the questionnaire measured explicit attitudes towards the consumption of red meat. It has been argued that the relations between explicit and implicit attitudes change when the category examples change (Nosek, 2005). The current approach was based on former studies that also assessed implicit attitudes towards an object (e.g. cigarettes) and explicit attitudes towards the behavior that entails the object (e.g. smoking a cigarette) (Huijding et al., 2005; Waters et al., 2007). Correlational analyses showed that the two types of attitudes were, although weakly, related in our study. Whether the relationship would have been even stronger with different target stimuli used in the SC-IAT, is currently unclear and should be investigated further. One possible way to minimize the incongruity between the explicit attitude and the implicit attitude could be to assess both attitudes towards the object, hence red meat. However, a pitfall would be that explicit attitudes are not congruent with the other explicit constructs social norms, social modeling, and self-efficacy anymore, which are always assessed towards a behavior. Another way could be the usage of stimuli in the implicit task that depict the consumption of red meat more clearly (e.g. a fork with a piece of red meat pointed towards the mouth). However, it might be questionable whether the subjects are able to recognize these stimuli clearly as the consumption of red meat as other factors might be disturbing (e.g. the fork, the mouth, the face(s) of people, etc.). Future research should test the best way to minimize the incongruity between the measured attitudes.

Third, the study was conducted online, thus participants were at home in an uncontrolled environment. This appears to be an environment more prone for distractions, which, one might expect, could especially affect the SC-IAT and its outcomes. However, Houben and Wiers (2008) investigated whether an Internet-delivered IAT would yield different results compared to an IAT performed in a lab and detected no significant differences. Importantly, they even found that the IAT performed at home was more strongly related to other explicitly assessed measures as well as to the target behavior (drinking behavior) than the lab-based IAT. This supported our choice to apply this method to our sample and setting as well. Additionally, we asked participants directly after having performed the SC-IAT whether they were distracted during the task. If this was the case, their data were excluded from the analyses.

Fourth, the SC-IAT showed low test-retest stability, which is a general issue faced by researchers using implicit measures. This might be due to sensitive systematic error (e.g., learning effects or situational cues (Bédard, 2000; Weir, 2005)) or might simply demonstrate a low validity of these measures. At this point in time, it is unclear if any of these two occurrences happened and were also responsible for the null-findings. These questions are an avenue for future studies. For example, after having performed the implicit measure multiple times, participants could get asked whether they noticed a learning effect, in order to control for possible learning effects.

Fifth, the study made use of an observational design. Although its longitudinal nature is definitely a pro, an experimental approach, in which dissonance would have been induced 
or the level of mindfulness would have been manipulated, would allow to draw stronger conclusions in terms of causation. We are convinced, however, that the present study offers a first step and ground for this topic leading to the next logical step - a replication in an experimental setting.

\section{CONCLUSION}

Mindfulness subskills are unassociated with the level of congruence between implicit and explicit attitudes toward red meat consumption. Instead, the mindfulness subskill accepting without judgment functions as a way to deal with dissonance. Future research should use this novel finding and investigate whether mindfulness can be used as a buffer in contexts where dissonance results in maladaptive behaviors. The exact relationship between attitude, attitude discrepancy, behavior, and intention appears to be complex and dependent on other variables, such as the type of behavior. To draw more generalizable conclusions, more research is needed to identify these behaviors and factors. 
Chapter 


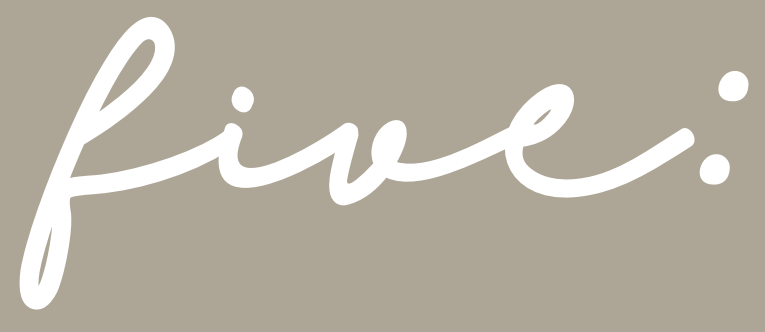

Does the discrepancy between implicit and explicit attitudes moderate the relationships between explicit attitude and (intention to) being physically active?

Muschalik, C., Elfeddali, I., Candel, M. J. J. M., Crutzen,R., \& de Vries, H. (2019). BMC Psychology, 7(1), 52. 


\section{A B S T R A C T}

Background: Explicit attitudes as well as implicit attitudes have been shown to be associated with physical activity (PA). These two types of attitudes can, however, be discrepant towards the same object or behavior. This study investigated whether there is a discrepancy between explicit and implicit attitudes (IED) regarding physical activity (PA), and whether IED moderates the relationship between explicit attitude and PA, and explicit attitude and PA intention.

Methods: At baseline (T0) and one (T1) and three months (T2) thereafter, students' ( $N=340)$ PA levels, intention, explicit attitudes, further PA determinants, e.g. selfefficacy, were assessed. Implicit attitudes towards PA were assessed by means of a tailored Single-Category Implicit Association task.

Results: IED was present but weak. Multiple hierarchical regressions revealed that IED did not moderate the relationship between explicit attitudes and PA or intention. Yet, IED was negatively associated with T0-PA and T1-PA.

Conclusion: The study revealed the important insight that IED is detrimental for PA. Interventions targeting attitudes to increase PA, should ensure that implicit and explicit attitudes regarding PA are concordant. 


\section{INTRODUCTION}

Explicit attitudes are a key construct in many behavioral theories and a relevant determinant across a wide range of health behaviors (Armitage \& Conner, 2001; Deforche, De Bourdeaudhuij, \& Tanghe, 2006; Glasman \& Albarracin, 2006; Kraus, 1995; Sheeran, Norman, \& Orbell, 1999). They are defined as conscious attitudes that are formed deliberately, which implies that people can self-report on their explicit attitudes (e.g. in a questionnaire). Explicit attitudes are composed of instrumental and affective components (Ajzen, 1991; Ajzen, 2002). Whereas the instrumental component refers to anticipated positive or negative consequences that would result from performing a behavior, the experiential component is understood as emotionladen judgments about a behavior. In recent decades, implicit attitudes have gained increased attention to serve as additional constructs for predicting and explaining health behaviors. They can be understood as mental associations between a concept (e.g. physical activity) and a favorable or unfavorable evaluation (e.g. positive or negative) (Devos, 2011) to which people do not have or sometimes do not want to have conscious access (Rydell \& McConnell, 2006). The strength of these associations manifests automatically into behavioral tendencies without the need for reflection. This has been demonstrated for a variety of behaviors (for example Conroy et al., 2010; Wiers, Van Woerden, Smulders, \& De Jong, 2002). To capture these associations, mostly reaction time paradigms are used. An example is the Implicit Association Test (IAT) in which participants have to sort words or pictures to given categories as quickly as possible (Greenwald et al., 1998). The underlying idea is that the stronger a negative or positive association in mind, the quicker is a person with categorizing the stimuli to the respective category. Based on that, inferences about the person's implicit attitude towards a specific object or behavior can be drawn. Dual-process models, such as the ReflectiveImpulsive Model (Strack \& Deutsch, 2004) or the Associative Propositional Evaluation Model (APE) (Gawronski \& Bodenhausen, 2006) depict that both explicit and implicit attitudes can be associated with behavior.

The relationship between behavior on the one hand, and implicit and explicit attitudes on the other hand, may however differ for different types of behaviors. For example, implicit attitudes are more strongly associated with spontaneous behavior and explicit attitudes with deliberate behavior (Hofmann et al., 2007; Neumann et al., 2004; Steffens \& Konig, 2006). For certain behaviors, such as voting behavior (Friese, Bluemke, \& Wanke, 2007) or physical activity (Conroy et al., 2010; Hyde et al., 2010), the two attitude-types can also have a joint effect. Furthermore, it has been shown that implicit and explicit attitudes towards one behavior do not always coincide: they can be discrepant, meaning that the explicit attitude towards a behavior is for example negative whereas the implicit attitude is positive or vice versa. This is called the implicit-explicit discrepancy (IED). In the study at hand, the effect of IED on the relationship between explicit attitude and behavior and explicit attitude and intention is investigated. 
The existence of IED has been demonstrated in several studies (Brinol et al., 2006; Petty et al., 2006; Rydell \& McConnell, 2006) and different factors have been discussed as possible sources for IED, such as self-presentational concerns (e.g. explicit measures are more likely to diverge from an implicit measure when self-presentational concerns are high) (Koole, Dijksterhuis, \& Van Knippenberg, 2001; Olson, Fazio, \& Hermann, 2007), preference for consistency (e.g. individuals with a stronger distinct motivation to seek congruence between their cognitions show lower IED compared to people with a less distinct preference for consistency) (Thrash et al., 2007), or methodological factors such as the consistency of the content assessed by the implicit and explicit measure (e.g. lower IED when the content of the measures is consistent) (Hofmann, Gschwendner, \& Schmitt, 2005). The APE also put forward theoretical assumptions about the existence of IED (Gawronski \& Bodenhausen, 2006). According to the APE, there exist two independent systems of reasoning. First, the slow-learning system, which operates by using interconnected associations in memory that are based on contiguity and similarity. Hence, learning takes place by the establishment of associations in memory that are formed slowly over time. Implicit attitudes are attributed to the slow-learning system. The second system, the fast-learning system, is assumed to rely on logic at a higher level of cognitive processing, which fits with the conceptualization of explicit attitudes and indicates that people can have control over the expression of their explicit attitudes and that they can be changed more quickly (Fazio, 1995). Hence, it is possible that a change in explicit attitude happens faster than a change in a person's implicit attitude, thereby resulting in dissonance between implicit and explicit attitudes (Rydell \& McConnell, 2006). Also, as implicit and explicit attitudes are ascribed to two different systems, they might be influenced by different processes. For example, in one study explicit attitudes were changed by means of verbally presented behavioral information whereas implicit attitudes were changed by subliminally presented primes (Rydell, McConnell, Mackie, \& Strain, 2006). When only one type of method is used, asymmetric changes can occur (Olson \& Fazio, 2006; Rydell \& McConnell, 2006) (e.g. when only one type of attitude is changed by means of a specific method that leaves the other attitude unaddressed), resulting in a dissonance between attitudes.

Although dissonance has repeatedly been demonstrated, only a few studies assessed the effect of IED on behavior (Brinol et al., 2006; Goldstein et al., 2014; Maliszewski, 2011; Petty et al., 2006; Rydell et al., 2008). Concerning this relationship, inconsistent patterns were found. For example, in a study on the consequence of discrepant attitudes on information processing Briñol et al. (2006) found that people with a greater discrepancy between their implicitly and explicitly measured self-dimensions, e.g. self-esteem, engaged in a more thorough elaboration of attitude-relevant information (but not of attitude-irrelevant information) than people with consistent self-dimensions. Also Rydell and colleagues (2008) demonstrated that diverging implicit and explicit attitudes towards a specific target person resulted in dissonance and in additional information processing about that person. The authors of both studies assumed that the higher information processing was a result of the participants' attempt to resolve the dissonance between the two attitudes, which is associated with negative feelings. In order to 
minimize these negative feelings, participants were motivated to engage in a more thorough information processing and to examine relevant information. In another study of Goldstein and colleagues (2014), IED positively predicted participants' chocolate consumption even when implicit and explicit attitudes were unrelated to the behavior. It was suggested that due to the discrepancy, the focus on the object (chocolate) was intensified and thereby increased the occurrence of disinhibited eating. These findings demonstrate that implicit and explicit attitudes can be in conflict, which in turn impacts behavior. Moreover, Karpen and colleagues (2012) revealed that the relationship between participant's explicit attitudes towards alcoholic beverages and alcohol consumption was moderated by IED. More precisely, explicit attitudes were not a significant predictor for alcohol consumption when IED was strong but a significant predictor when IED was low.

Also in the context of physical activity, it has been shown that IED exists and that it impacts behavior (Berry, Rodgers, Divine, \& Hall, 2018; Brand \& Antoniewicz, 2016). For example, the lower IED was in a sample of fitness club exercisers, the more successful they were in achieving their ideal exercise frequency (Brand \& Antoniewicz, 2016). In another study, discrepancy between explicit and implicit health measures regarding PA was negatively associated with the length to participate in a one year long exercise program (Berry et al., 2018). These findings demonstrate that there is a direct link between IED and PA behavior. It is, however, unclear whether IED also acts as a moderator of the relationships between explicit attitude and physical activity (PA) (as it was the case in the study of Karpen et al. (2012) regarding alcohol consumption) and explicit attitude and intention.

New insights into these effects can help to understand the way implicit and explicit attitudes jointly guide PA, and thereby provide input to improve interventions that are aiming to enhance people's activity levels. PA behavior has been addressed by means of numerous health interventions (Knittle et al., 2018), as increased activity is known to have significant health benefits (World Health Organization, 2017). Yet, around 23\% of the global adult population (World Health Organization, 2017) and $80 \%$ of the global adolescent population (Ferreira de Moraes, Guerra, \& Rossi Menezes, 2013; World Health Organization, 2017) are not sufficiently active, thereby increasing their risk for the development of noncommunicable diseases, such as cardiovascular diseases or diabetes (World Health Organization, 2017). Therefore, more insight into additional influencing factors, such as the effect of IED, could be helpful. Thus far, research has found that social-cognitive determinants such as a more positive explicit attitude towards PA, stronger perceived norms (i.e. the perceived norm that one should be active), stronger modeling (i.e. perceiving significant others in one's environment as active), and more self-efficacy (i.e. perceiving oneself as capable of performing the behavior even in difficult situations) lead to a higher intention to be physically active (Hagger et al., 2002; Reynolds et al., 1990; Sallis et al., 1986; Schulz et al., 2014; Smeets et al., 2007). Although intention does not always result in the translation of behavior - a phenomenon called the intention-behavior gap - it is one of the most proximate determinants of behavior and vital in the process of initiating a behavior (Sheeran \& Webb, 2016). Also regarding PA, a higher intention is more 
likely to result in PA behavior (De Vries, 2017; Downs \& Hausenblas, 2005; Fishbein \& Ajzen, 2011; Hagger et al., 2002). Moreover, a more positive explicit attitude towards PA does not only result in a higher intention but also in greater PA levels (Deforche et al., 2006; Graham, Sirard, \& Neumark-Sztainer, 2011; Sallis et al., 1986). Studies concerning the impact of explicit cognitions mostly concern the effects of explicit attitude which have been found to explain around $30 \%$ of variance in PA intention (Biddle \& Mutrie, 2007). Therefore, explicit attitudes have been classified as an important and central predictor for PA engagement (Biddle \& Mutrie, 2007; Ciccomascolo \& Grossi, 2008; Godin, 1994; Graham et al., 2011; SalehNia, Mizany, Sajadi, \& Rahimizadeh, 2012) and it is recommended that interventions reinforce attitude change in order to facilitate PA engagement and adherence (Chatzisarantis et al., 2005). In recent studies, also implicit attitudes were shown to be associated with PA levels (Bluemke et al., 2010; Brand \& Schweizer, 2015; Conroy et al., 2010; Hyde et al., 2010). For example, exercisers hold more positive automatic associations towards PA than non-exercisers (Bluemke et al., 2010) and implicit attitudes predict PA behavior above and beyond the aforementioned social-cognitive determinants (Conroy et al., 2010). Moreover, a former study showed that implicit attitudes moderate the relationships between certain explicit cognitions (i.e. perceived cons, selfefficacy) and intention as well as between certain explicit cognitions (i.e. self-efficacy) and PA behavior (Muschalik, Elfeddali, Candel, \& de Vries, 2018). The present study extends the previous study and adds new insights into the influence of IED on the relationship between explicit attitude and intention/PA behavior.

Until now, it is clear, that explicit attitudes play, besides other explicit cognitions (social norms, social modeling, self-efficacy) and implicit attitudes, a significant role in the prediction of PA. It remains unclear however, whether explicit attitudes are still strongly associated with PA behavior when explicit attitudes are discrepant from the implicit attitude (which is also associated with PA). Karpen et al. (2012) demonstrated that high IED weakens the predictive power of explicit attitudes regarding behavior and argued that as a result of the discrepancy, the information regarding the target (behavior) are inconsistent. This in turn makes it harder for the individual to judge about and to move towards the target behavior. Based on this, we first explored whether IED is present in our sample and we expect it to be existent (Hypothesis 1). Secondly, we investigated whether the predictive power of explicit attitudes regarding PA behavior is also moderated by IED. We expected IED to moderate the relationship between explicit attitudes and PA behavior with explicit attitudes being a stronger predictor for PA behavior when IED is low and a weaker predictor for PA when IED is high (Hypothesis 2[H2]; Figure 3.1).

According to social-cognitive models, explicit attitudes are strongly associated with intention. Intention does not always translate into actual behavior (Sheeran \& Webb, 2016), however, it is the most proximal determinant for (PA) behavior (De Vries et al., 2005; Fishbein \& Ajzen, 2011; McEachan et al., 2011; Sheeran et al., 2017). As high IED has shown to weaken the effect of explicit attitude on behavior (Karpen et al., 2012), we argue that high IED should also weaken the effect of explicit attitude on intention. Hence, on top of the second hypothesis, 
we investigated whether IED also moderates the relationship between explicit attitude and intention. We expected that the relationship between explicit attitude and intention is moderated by IED with greater IED leading to a weaker relationship between explicit attitude and intention and lower IED leading to a stronger relationship between explicit attitude and intention (Hypothesis 3 [H3]; Figure 3.1). Gaining insight into these effects could help to understand whether interventions aiming to increase PA intention and behavior by changing or fostering explicit attitudes have to take discrepant attitudes into account.

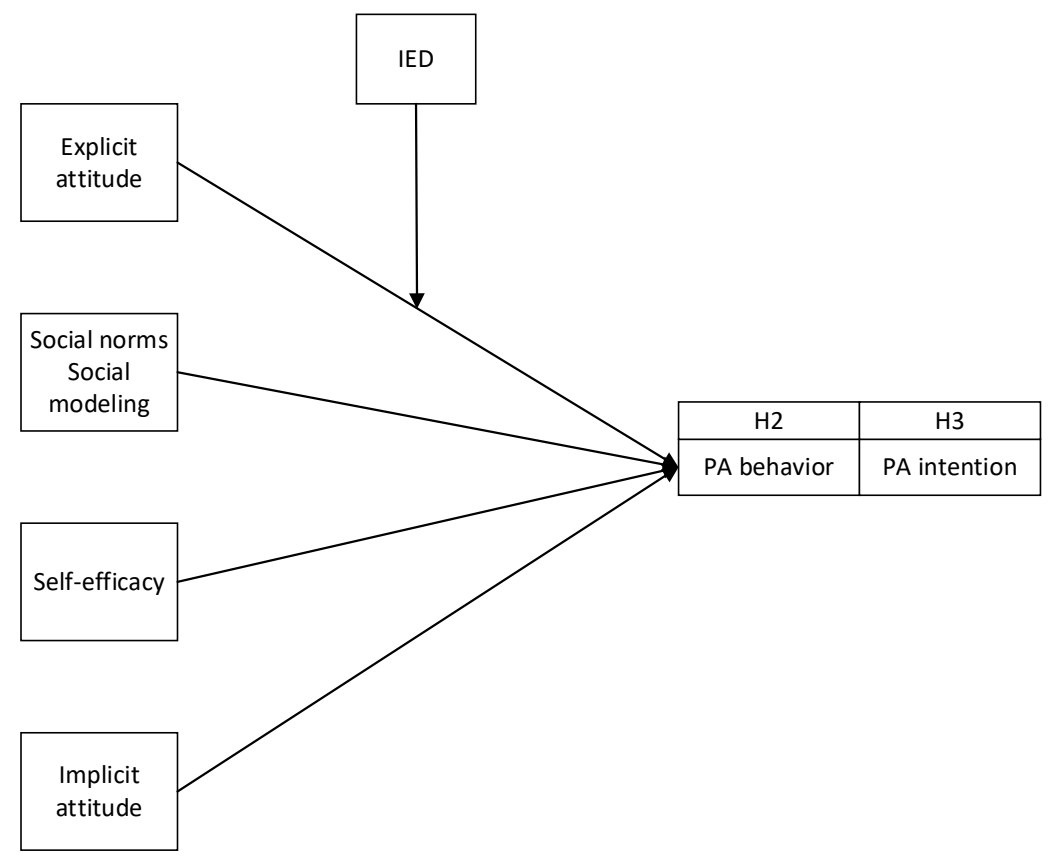

Figure 3.1 | Does IED moderate the relationship between explicit attitude and PA behavior $(\mathrm{H} 2)$ and the relationship between explicit attitude and PA intention $(\mathrm{H} 3)$ ?

\section{METHODS}

\section{Design}

The study at hand is part of a larger study in which we investigated how implicit attitudes synergistically interact with explicit cognitions in the prediction of PA intention and behavior (Muschalik et al., 2018). In the current study, the emphasis is on the moderating effect of IED. A three-wave longitudinal study was conducted with measurements at baseline (T0) and followups after one month (T1) and after three months (T2). 


\section{Ethical approval}

Ethical approval for this study was obtained from the Medical Ethics Committee of Zuyderland (METC Z.), the Netherlands (15-N-169).

\section{Participants and recruitment}

The study was conducted at the Behavioral and Experimental Economics Laboratory (BeeLab) of a Dutch University. The BeeLab holds a database of students who are willing to participate in experiments, which was used to recruit participants for this study. Most students in the database were German or Dutch native speakers and, therefore, the study was conducted in both these languages. If a student had indicated German or Dutch as mother tongue, then he or she was invited to participate via email. No further inclusion criteria needed to be met. At baseline, 1690 students were invited out of which 340 participated (i.e. 20\% response rate). The low response rate could be explained by the fact that the subject pool is not updated regularly and thus also contains students who are finished with their studies. Also the requirement to come twice to the lab within a period of one month in order to receive one's incentive might have been a barrier for participation. At T1, 240 students participated and after three months, 128 students took part.

\section{Procedure}

All students who met the inclusion criteria of having German or Dutch as mother tongue received an invitation via email stating the subject of the study (i.e. physical activity and related cognitions). Further, students were informed about the three waves of measurement and that each measurement consisted of two tasks, which together took around 25 minutes to complete. Further, it was explained that there were no risks related to the participation and that all data would be gathered and analyzed anonymously. For the completion of the first two measurements, students received $€ 15$ and another $€ 7.50$ when having completed the third measurement. When willing to participate, students could choose their preferred timeslot on two given days. An e-mail reminder was sent one day before participating. On the day of participation, participants were welcomed in the lab, received instructions and provided written informed consent. To assess their implicit attitudes towards PA, they first completed a Single-Category Implicit Association Test (SC-IAT; Karpinski \& Steinman, 2006) and subsequently filled in a questionnaire to obtain their explicit attitude. Since we know that PA intention and behavior are also strongly associated with social norms, social modeling and self-efficacy (Hagger et al., 2002; Hausenblas, Carron, \& Mack, 1997), we assessed these constructs as well in order to be able to demonstrate the effect of IED on intention and behavior, independent of these other cognitions. The questionnaire had to be completed after the SCIAT as it is expected that prior questions about PA would trigger related thoughts and could thereby affect the reaction time during the SC-IAT (Bargh et al. 2000). In the questionnaire, the following constructs were assessed in the following order: explicit attitude comprised of perceived pros and perceived cons, social norms and social modeling, self-efficacy, intention, and physical activity levels. 


\section{Measurements}

Implicit attitude assessment task. Implicit attitudes towards PA were assessed by using the SCIAT which showed adequate internal reliability and predictive validity in previous studies (Hyde et al., 2010; Karpinski \& Steinman, 2006; Rebar, Ram, \& Conroy, 2015). Additionally, the SC-IAT was used in former studies in which it successfully predicted objectively-measured PA (Conroy et al., 2010) as well as unintentional PA (Conroy et al., 2010; Hyde et al., 2010).

In the computerized tasks, participants were asked to indicate as fast and accurately as possible whether presented stimuli belonged to one of two given categories. The task consisted of two blocks which contained 24 practice trials and 72 test trials. In one block 'physical activity or negative' built a category and 'positive' the other category. In the other block, categories were reversed, thus 'physical activity or positive' was one category and 'negative the other. The underlying assumption is that the stronger an implicit association is, the faster the reaction. Hence, if a person has negative implicit associations with being physically active he or she would be quicker in categorizing the displayed stimuli when 'physical activity or negative' are one category than when 'physical activity or positive' build a category. To ensure that reaction times were not influenced by the order of the blocks, the order of the two blocks was counterbalanced. Thus some participants had the categories 'physical activity or positive' and 'negative' first and the reversed categories subsequently, whereas other participants had the block 'physical activity or negative' and 'positive' first and the reversed pattern afterwards. Throughout the whole SC-IAT, labels for the two categories were displayed on the left or right upper part of the screen. If the presented stimulus belonged to the category that was displayed on the left part of the screen, participants had to press $e$ on their keyboard. When the stimulus belonged to the category that was presented on the right upper part of the screen, the participant had to press $i$ on the keyboard. The words were presented in a random order and equally frequent. In case that an incorrect answer was given, a red X appeared on the screen until the participant corrected the answer as recommended by Greenwald et al. (2003).

The selection procedure for the stimuli was as follows: based on their valence and arousal norms, positive and negative words were chosen from the Affective Norms for English Words (ANEW) (Bradley \& Lang, 1999). Words representing PA were selected from the studies of Conroy et al. (2010) and Hyde et al. (2010) who also used the SC-IAT to measure implicit attitudes towards PA. All selected words were translated forth and back from English to Dutch and German by native speaking researchers of the University. The positive and negative words were then pretested regarding the perceived levels of valence $(1$ = very negative to 9 = very positive), arousal ( 1 = not arousing at all to $9=$ very arousing), and familiarity ( 1 = very unfamiliar to $9=$ very familiar). PA related words were pretested regarding their representativeness for PA ( $1=$ not representative at all, 2 = not so strongly/a bit representative, 3 = strongly representative). The pre-test was done among 26 German and 22 Dutch native students of the University. Love, freedom, joy, success, and party were selected as positive words (translated from German and Dutch); depression, demon, lie, infection, and poison were 
selected as negative words (translated from German and Dutch). The seven words running, biking, kickboxing, sprint, jogging, lifting weights, and sit-ups were selected as words to represent PA (translated from German and Dutch).

By means of the Inquisit Millisecond 4.0 software (Inquisit, 2015), the SC-IAT was programmed and presented. The script was based on the manual of Karpinski and Steinman (2006). The implicit attitude was indicated by $d$-scores that were calculated by the software using the improved scoring algorithm as described by Greenwald et al. (2003). In this procedure, the average response time for the test block with the categories physical activity or negative/ positive is subtracted from the average response time of the reversed test block, in this case physical activity or positive/negative. Afterwards, the score gets divided by the standard deviation of all correct response times of the test trials. Normally, $d$-scores range from -2 to 2. Reaction times of our sample did not exceed this range. Positive scores indicate a positive implicit attitude and negative scores indicate a negative implicit attitude. The higher the score, the more positive the implicit attitude. Based on the procedure as described in Karpinski and Steinman (2006) we assessed the internal reliability of the SC-IAT by dividing the SC-IAT into thirds (blocks of 24 test trials) and calculated a separate $d$-score for each third. A measure of internal consistency was obtained by calculating the average intercorrelation among these scores and applying the Spearman-Brown formula that revealed an acceptable value of $r=.83$. Test-retest correlation between $\mathrm{d}$-scores at baseline and at T1 showed a significant moderate correlation of $r=.43(p<.001)$ and test-retest correlation between $d$-scores at T1 and at T2 showed a low correlation of $r=.17(p=06)$. Latter result is comparable to the results of other studies, which demonstrated weak test-retest reliabilities for the SC-IAT regarding other topics (Stieger, Göritz, \& Burger, 2010) as well as in the context of PA (Hyde et al., 2012).

Questionnaire. The questions to assess explicit cognitions were based on the I-change model (De Vries, 2017; De Vries et al., 2005), which was used in former studies to assess PA related cognitions (De Vries et al., 2008; Schulz et al., 2014; Smeets et al., 2007). The following definition of adequate PA was shown to the participants with the option to reread it an an time while answering the questionnaire: Being sufficiently active is defined as being moderately physically active five times a week for at least 30 minutes. Being moderately active means an increase in heart rate that is induced by activities such as brisk walking (Health Council of the Netherlands, 2017). The full questionnaire can be found at https://doi.org/10.1186/s40359018-0229-0.

Explicit attitude was assessed on a 5-point Likert Scale with two scales measuring perceived pros and perceived cons, each expressed by 10 statements. Pros were measured by affective items such as 'Being adequately physically active is' (1) 'very enjoyable' to (5) 'not enjoyable', and instrumental items such as 'Being adequately physically active is' (1) 'very good for my health' to (5) 'not good for my health'. Items were reversed, so that higher items represent the perception of more advantages. Based on low factor loadings, three items from the pro scale were removed $(\Omega=.75)$. Perceived cons were measured by affective items such as 'Being adequately physically active is' (1) 'very unpleasant' to (5) 'not unpleasant', and instrumental 
items such as 'Being adequately physically active is' (1) 'too expensive' to (5) 'not expensive'. Lower scores indicate the perception of fewer disadvantages. Three items were removed from the scale, also due to low factor loadings $(\Omega=.70)$. For the analysis, a sum score for the con scale and a sum score for the pro scale were created. Both scale scores were added to represent one scale score for explicit attitude (range 14-70) that was used in the analyses. The higher the score, the more positive the explicit attitude.

Social norms and social modeling were each assessed by four questions on a 5-point Likert scale. Whereas norm items assessed expectations of family members, partners, and friends, with respect to PA, modeling items assessed the PA behavior of those. An example for a social norm item is 'My partner' (1) 'doesn't expect me at all to be physically active' to (5) 'certainly expects me to be adequately physically active'. An example for a modeling item is 'Most of my family members are adequately physically active' with answers ranging from (1) 'totally disagree' to (5) 'totally agree'. The mean score for norms was included in the analyses $(\Omega=.62)$. The higher the score, the stronger the norms. Factor saturation regarding social modeling was estimated as insufficient $(\Omega=.34)$, which was also demonstrated by low factor loadings. Hence, social modeling items were included separately in the analyses.

Self-efficacy was measured on a 5-point Likert scale. Nine statements asked participants to indicate to what extent they expect themselves to be able to be adequately physically active in different situations, for instance 'I find it difficult/easy to be adequately physically active when I am very busy' with answers ranging from (1) 'very difficult' to (5) 'very easy'. Based on their low factor loadings and their content (i.e. items referring to a specific activity instead of physical activity in general), three items were removed and a mean scale score was created of the remaining six items and included in the analyses $(\Omega=.66)$. A higher score indicates higher levels of self-efficacy.

Three items measured a person's intention to be adequately active. On a 5-point Likert scale the first item assessed whether respondents were planning to be adequately physically active within the next three months ranging from (1) 'no, not at all' to (5) 'yes, absolutely'. The second item asked whether respondents were motivated to be adequately physically active within the next three months with answer options from (1) 'totally disagree' to (5) 'totally agree', and the third item assessed how high chances were to be adequately physically active within the next three months with answers ranging from (1) 'very little' to (5) 'very high'. The mean score of all three items was included as scale score for intention in the analyses $(\Omega=.89$ ) with higher scores representing a stronger intention.

Physical activity levels were assessed by using the Short Questionnaire to Asses Healthenhancing physical activity (SQUASH) (Wendel-Vos et al., 2003). The SQUASH has been used in former studies to assess PA (De Vries et al., 2008; Schulz et al., 2014; Smeets et al., 2007) and the reliability and validity were demonstrated (RIVM, 2005; Wendel-Vos et al., 2003). The SQUASH assesses different activities (e.g. commuting activities, household activities, leisure time activities). For each activity the frequency, duration (in minutes), and intensity (light/moderate/ intense expressed in metabolic equivalent values, METs) were assessed. Total minutes of an 
activity were calculated by multiplying the frequency of an activity by its duration. The total minutes in turn were multiplied by the respective intensity in order to get an activity score for each activity (Wendel-Vos et al., 2003). By the sum of all different activity scores, a total activity score was obtained. The higher the score, the more active a person is.

Further, age ('How old are you?') and gender ('What is your gender?') were assessed and included in the analyses. Also participants were asked whether they were unable to be currently physically active in the recent past due to an illness ('Do/did you suffer from an illness that makes/made it impossible for you to be physically active, e.g. brain bleeding or cancer?'). As none of the participants answered the question with 'yes', data of all participants were included.

\section{Analyses}

All analyses were done with SPSS version 23. In advance, differences between the German and Dutch versions of the tests were tested but not detected. In order to assess scale quality of the measurements used in the present study we calculated their dimensionality by means of exploratory factor analyses as well as McDonald's omega as a less biased alternative to Cronbach's alpha (Crutzen \& Peters, 2017). Based upon the sum of the squared loadings

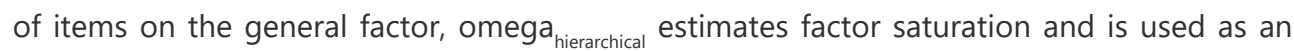
indicator of internal structure (McDonald, 2013). Values were calculated with the R program ( $R$ Core Team, 2016) and are displayed in the measurements section above.

To evaluate the effect of IED, an index was created by calculating the absolute value of the difference between the average of a participant's standardized explicit attitude score and the standardized reaction times of the SC-IAT. This procedure is based on a number of previous studies on IED (Brinol et al., 2006; Petty, Briñol, \& DeMarree, 2007; Rydell et al., 2008). The index indicates where participants fall within the distribution of the sample on the explicit versus implicit measure, thus demonstrating the size of the discrepancy. When a person's place in the distribution is the same on the explicit and implicit measure (e.g. low in the distribution on both measures, high in the distribution on both measures, and so on), the index has a value close to zero. The more the attitudes deviate from each other (e.g. low in the distribution of implicit attitudes and high in the distribution of explicit attitudes and vice versa), the higher the score on the index and the further away it is from zero. For an indication of the reliability of the IED index, we created three indices and conducted test-retest correlations between the indices that were created for the measurements at baseline and after one and three months. The baseline index showed a moderate correlation with the index after one month $(r=.52, p<$ .001) and a weak correlation with the index after three months $(r=.29,<.001)$.

For the second hypothesis and in order to assess cross-sectional and longitudinal effects of the moderating effect of IED on the relationship between explicit attitude and PA, three regressions were conducted. For short-term effects, we regressed participant's PA levels at T0 on age and gender in step one, baseline explicit attitudes, social norms, social modeling, selfefficacy, implicit attitude, and IED in a second step, and added the interaction between IED and 
explicit attitude in a third step. To assess long-term effects, the same regression was repeated but with PA at T1 and T2 as dependent variable. When the interaction between explicit attitude and IED was significant, we conducted stratified analyses with IED.

To investigate the third hypothesis and short-term and long-term effects of IED on the relationship between explicit attitude and intention, we conducted three regressions each with intention at baseline, at $\mathrm{T} 1$ and at $\mathrm{T} 2$ as dependent variable. Baseline variables were again added in three steps of a regression. Age and gender in step one, explicit attitudes, social norms, social modeling, self-efficacy, implicit attitudes and IED in step two, and the IED by explicit attitude interaction in step three. Main effects of the regression analyses were interpreted in the second step of the regression and the two way interaction in the third step (Cohen, Cohen, West, \& Aiken, 2013). Cases with missing values were not included in the analyses.

\section{RESULTS}

\section{Characteristics of the sample}

The mean age of the sample at baseline $(N=340)$ was 21 years $(S D=2.11)$ and $61 \%$ was female. Of the sample, $91 \%$ met the Dutch Guideline for physical activity, which was, at the time the study was conducted, to perform moderate or vigorous activities for at least 150 minutes per week (Health Council of the Netherlands, 2017). After one month, 240 students participated $(71 \%$ of baseline, $64 \%$ female, mean age $=21, \mathrm{SD}=2.12)$ and after three months, 128 students ( $38 \%$ of baseline, $69 \%$ female, mean age $=22, \mathrm{SD}=2.17$ ) took part. At follow-up one and two, more men dropped out than women (T1: OR $=0.55 .95 \% \mathrm{Cl}[.04,1.00] . \mathrm{p}=.02$; T2: $\mathrm{OR}=0.51,95 \% \mathrm{Cl}[.02,1.00], \mathrm{p}=.01)$. No other variables predicted dropout. We included gender, a significant predictor of dropout, in all further analyses.

Table 3.1 | Means, standard deviations and correlations between study variables at baseline.

\begin{tabular}{|c|c|c|c|c|c|c|c|c|c|c|c|}
\hline & \multirow[t]{2}{*}{$M(S D)$} & \multicolumn{10}{|c|}{ Correlations } \\
\hline & & 1 & 2 & 3 & 4.1. & 4.2. & 4.3. & 4.4 & 5 & 6 & 7 \\
\hline 1. Explicit attitude & $56.30(6.22)$ & & & & & & & & & & \\
\hline 2. Implicit attitude & $.1160(.3310)$ & $.11^{\star}$ & & & & & & & & & \\
\hline 3. Social norms & $3.89(.74)$ & .07 & .01 & & & & & & & & \\
\hline 4.1. Social modeling (partner) & $3.48(1.20)$ & .08 & .06 & $.34^{\star *}$ & & & & & & & \\
\hline 4.2. Social modeling (family members) & $3.43(1.13)$ & $.26^{\star \star}$ & .01 & $.15^{\star \star}$ & .10 & & & & & & \\
\hline 4.3. Social modeling (friends) & $3.55(.91)$ & $.12^{\star}$ & .07 & $.18^{\star *}$ & $.33^{* *}$ & $.13^{*}$ & & & & & \\
\hline 4.4. Social modeling (colleagues) & $3.31(.83)$ & $.11^{\star}$ & .08 & $.15^{\star *}$ & .15 & $.13^{*}$ & $.44^{* *}$ & & & & \\
\hline 5. Self-efficacy & $2.60(.62)$ & $.45^{\star *}$ & .08 & -.09 & .12 & $.14^{\star}$ & .02 & .03 & & & \\
\hline 6. Intention & $4.43(.67)$ & $.57^{\star \star}$ & .06 & .08 & .11 & $.21^{\star \star}$ & $.24^{\star *}$ & $.19^{\star \star}$ & $.40^{\star \star}$ & & \\
\hline 7. Physical activity & 4959.03 (3187.16) & $.24^{\star *}$ & .07 & .02 & .02 & .06 & .04 & .02 & $.20^{\star \star}$ & $.24^{\star \star}$ & \\
\hline 8. IED & $1.06(.81)$ & -0.10 & .02 & .04 & -.005 & -.05 & .04 & .06 & -.04 & -.10 & .01 \\
\hline
\end{tabular}




\section{Associations between predictors}

Descriptive statistics and correlations between the study variables at baseline are presented in table 3.1. IED had a range of 0.00-4.29 $(M=1.06, S D=.81)$ and the mean differed significantly from zero ( $\mathrm{t}=$ (339) 24.27, $\mathrm{p}<.001$ ). The distribution of IED scores at baseline is displayed in figure 3.2. IED was not correlated with any of the measured explicit cognitions. Explicit attitudes and implicit attitudes were significantly correlated with each other $(r=.11)$. Also, explicit attitude was correlated with intention, self-efficacy, social modeling by family members, PA, and social modeling by colleagues and friends. Implicit attitudes were not significantly correlated to any other explicit cognitions.

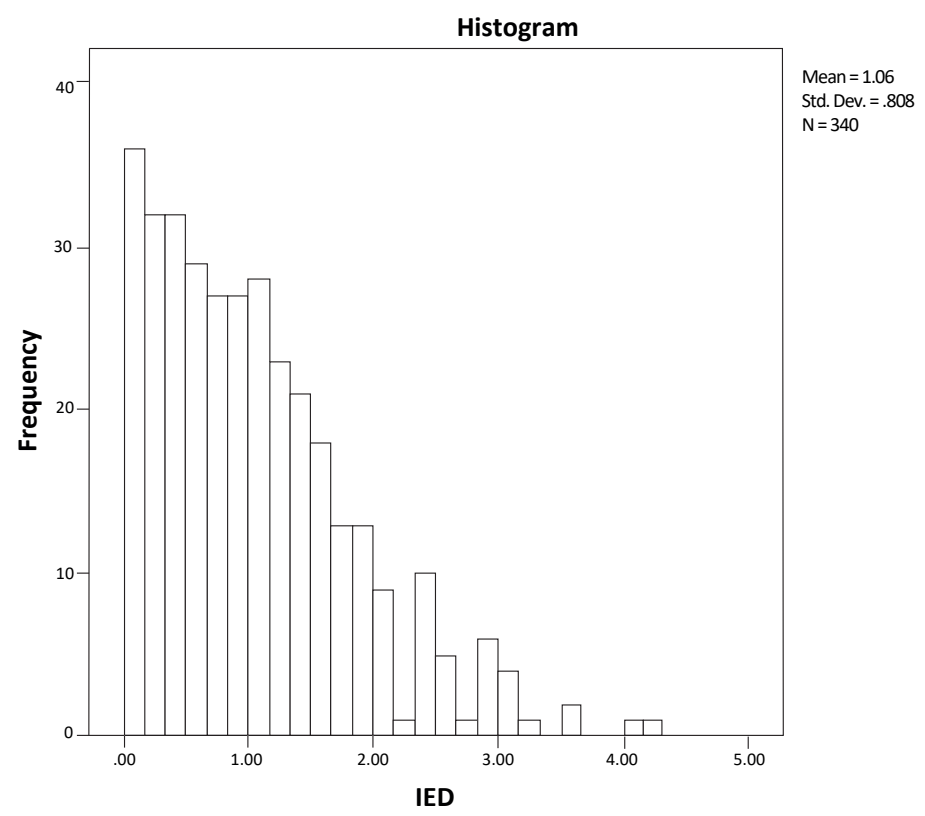

Figure 3.2 | Distribution of IED at baseline $(\mathrm{N}=340)$.

Does IED moderate the relationship between explicit attitude and PA behavior (at TO, T1, and T2)?

The interaction between IED and explicit attitude was not significant for PA at T0 $(\beta=.004$, $p=.97,95 \% \mathrm{Cl}[-73.55,76.45])$ nor at T1 $(\beta=-.03, p=.85,95 \% \mathrm{Cl}[-106.06,87.96])$ or at T2 $(\beta=.04$, $p=.87,95 \% \mathrm{Cl}([-124.42,146.91])$. PA at T0 was significantly associated with self-efficacy $(\beta=.22$, $p=.02,95 \% \mathrm{Cl}[132.39,1622.10])$ and IED $(\beta=-.16, p=.05,95 \% \mathrm{Cl}[-1072.79,8.78])$, demonstrating that a higher IED is associated with lower PA levels.

At T1, PA was significantly associated with self-efficacy $(\beta=.38, p<.001,95 \% \mathrm{Cl}[706.49$, $2668.20])$ and with IED $(\beta=-.20, p=.06,95 \% \mathrm{Cl}([-1397.93,40.93])$, also indicating that a high IED is associated with less PA. 
After three months, PA was again significantly related to self-efficacy $(\beta=.43, p=.005,95 \% \mathrm{Cl}$ $[539.93,2801.46])$, but not with IED $(\beta=-.17, p=.24,95 \% \mathrm{Cl}[-1470.75,382.83])$. The results for all predictor variables are displayed in table 3.2.

\section{Post-hoc analyses}

As a result of the null-findings, we conducted post-hoc analyses, in which we tested whether the relationship between the affective explicit attitude and PA is moderated by the discrepancy between the affective explicit attitude and the implicit attitude. This is based on the assumption that implicit attitudes are grounded in affective associations and therefore rather comparable to affective explicit attitudes than to instrumental explicit attitudes (Brand \& Antoniewicz, 2016). Hence, the question arises whether the discrepancy between these two constructs might influence the effect of the affective explicit attitude on PA behavior. We conducted the same three regression analyses as earlier, however instead of adding an overall explicit attitude score in step 2, we added affective explicit attitude and instrumental explicit attitude as single predictors, and instead of an index for the discrepancy between the overall explicit attitude (comprised of the affective and the instrumental dimension) and implicit attitudes, we added IED (affective) - an index for the difference between the implicit attitude and the affective explicit attitude only. In a third step, the interaction between IED (affective) and the affective explicit attitude was added. The other variables (e.g. self-efficacy, social norms) were added in the same steps as in the earlier regressions. PA at baseline and after one and three months served each as dependent variable.

At no measurement, the interaction between IED (affective) and the affective explicit attitude was significant (baseline: $\beta=-.09, p=.43,95 \% \mathrm{Cl}[-227.14,97.42] ; \mathrm{T} 1: \beta=-.07, p=.62$, 95\% Cl [-257.33, 154.77]; T2: $\beta=-.02, p=.94,95 \% \mathrm{Cl}[-318.27,295.07])$. At baseline and T1 however, IED (affective) was significantly associated with PA (baseline: $\beta=-.19, p=.03,95 \% \mathrm{Cl}$ $[-1136.26,-54.08] ; \mathrm{T} 1: \beta=-.24, p=.04,95 \% \mathrm{Cl}[-1556.51,-41.76])$, indicating that a greater discrepancy between the implicit attitude and the affective explicit attitude is associated with lower PA levels. The same pattern was found in the earlier analyses when IED was comprised of the discrepancy between both the affective and instrumental dimension of the explicit attitude and the implicit attitude.

Moreover, as IED was significantly associated with PA at baseline and T1, we tested whether the direction of the discrepancy plays a role in this regard or not. To do so, we conducted two additional regressions in which we added age and gender in step 1, explicit attitude, social norms, social modeling, self-efficacy, implicit attitude, and IED in step 2, and the direction of the dissonance (coded as dummy) as well as an interaction term between IED and the direction of the dissonance in step 3. For PA at baseline, the interaction term was not significant $(\beta=1.28, p=.10,95 \% \mathrm{Cl}[-1033.23,11636.43])$. For PA at $\mathrm{T} 1$, the interaction was significant $(\beta=2.55, p<.001,95 \% \mathrm{Cl}[2698.91,17946.37])$ and additional simple slope analyses showed that IED was significant when the explicit attitude was higher/more positive than the implicit attitude $(\beta=1.76, p=.04,95 \% \mathrm{Cl}[466.83,12152.93])$ but not vice-versa $(\beta=-.51, p=.60$, $95 \% \mathrm{Cl}[-7058.48,4158.91])$. 


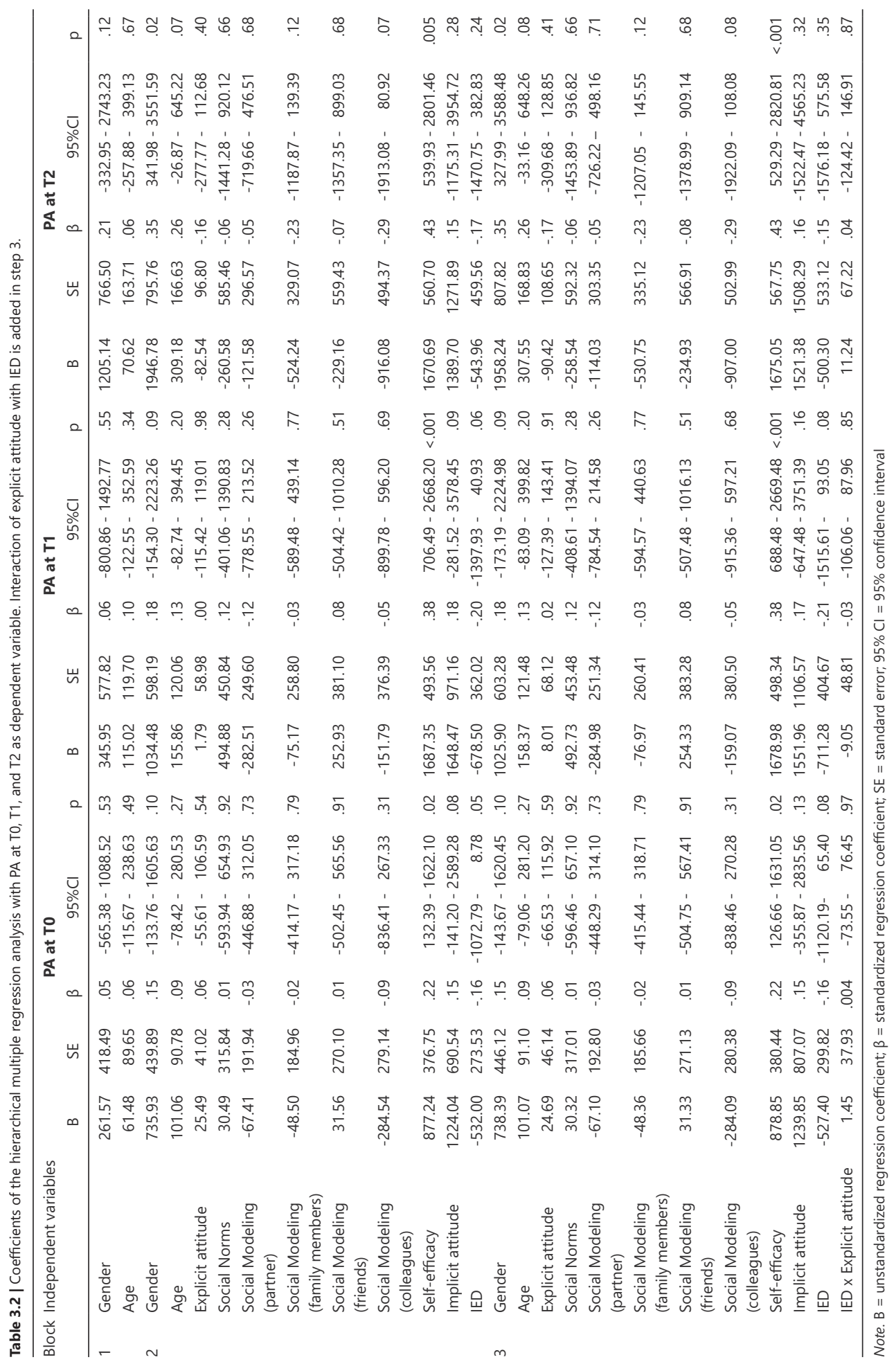




\section{Does IED moderate the relationship between explicit attitude and the intention to be active (at TO, T1, and T2)?}

No significant interaction between IED and explicit attitude was found for intention at T0 $(\beta=.03, p=.74,95 \% \mathrm{Cl}[-.01, .02])$. Explicit attitude $(\beta=.43, p<.001,95 \% \mathrm{Cl}[.03, .06])$ and selfefficacy $(\beta=.23, p=.007,95 \% \mathrm{Cl}[.06, .39])$ were significantly associated with T0 intention.

For intention at $\mathrm{T} 1$, the interaction between IED and explicit attitude was also not significant $(\beta=.19, p=.17,95 \% \mathrm{Cl}[-.01, .04])$. Significant predictors were explicit attitude $(\beta=.20, p=.05$, $95 \% \mathrm{Cl}[.00, .85])$, social modeling of family members $(\beta=-.19, p=.05,95 \% \mathrm{Cl}[-.22, .001])$, and self-efficacy $(\beta=.26, p=.01,95 \% \mathrm{Cl}[.06, .48])$.

At $\mathrm{T} 2$, explicit attitude $(\beta=.58, p=.002,95 \% \mathrm{Cl}[.02, .10])$ and social modeling (partner) $(\beta=.31, p=.02,95 \% \mathrm{Cl}[.03, .25])$ were significantly related to intention. The interaction between IED and explicit attitude was not significant $(\beta=-.03, p=.89,95 \% \mathrm{Cl}[-.03, .02])$.

\section{Post-hoc analyses}

As a result of the null-findings, we conducted post-hoc analyses, similar to the ones performed regarding question 2 . This time we tested whether the relationship between the affective explicit attitude and intention is moderated by the discrepancy between the affective explicit attitude and the implicit attitude. This is based on the same reasoning that implicit attitudes are grounded in affective associations and therefore rather comparable to affective explicit attitudes (Brand \& Antoniewicz, 2016). The same regressions as earlier were conducted. Instead of an overall explicit attitude score, we added affective explicit attitude and instrumental explicit attitude as single predictors in step 2, and instead of an index for the discrepancy between the overall explicit attitude (comprised of the affective and the instrumental dimension) and implicit attitudes (IED), we added IED (affective) as an index for the difference between the implicit attitude and the affective explicit attitude. In a third step, the interaction between IED (affective) and the affective explicit attitude was added. The other variables (e.g. self-efficacy, social norms) were added in the same steps as in the earlier regressions. Intention at baseline and after one and three months served each as dependent variable.

At no measurement, the interaction between IED (affective) and the affective explicit attitude was significant (baseline: $\beta=-.001, p=.99,95 \% \mathrm{Cl}[-.04, .04] ; \mathrm{T} 1: \beta=.15, p=.28,95 \% \mathrm{Cl}[-.02, .07]$; $\mathrm{T} 2: \beta=-.13, p=.52,95 \% \mathrm{Cl}[-.08, .04])$. The same pattern was found in the earlier analyses when IED was comprised of the discrepancy between both the affective and instrumental dimension of the explicit attitude and the implicit attitude. 


\section{DISCUSSION}

The current study is part of a larger study, which showed that explicit cognitions and implicit attitudes interact in the prediction of PA behavior and intention (Muschalik et al., 2018). Previous studies have shown that explicit attitudes and implicit attitudes can be discrepant from each other (Brinol et al., 2006; Petty et al., 2006; Rydell and McConnell, 2006; Rydell et al., 2008; Goldstein et al., 2014; Maliszewski. 2011) and explicit attitudes can be a weaker predictor for behavior when this is the case (Karpen et al., 2012). The present study aimed to extend this idea with physical activity as target behavior, thereby adding new insights into two specific relationships and the role of IED in these relationships (Muschalik et al., 2018). More precisely, we investigated the effect of IED on the influence of explicit attitudes on PA behavior as well as on intention.

IED was present but not very strong. Contrary to our hypotheses and former research (Karpen et al., 2012) we did not find IED to moderate the relationship between explicit attitude on PA behavior at any of the three measurements. Although PA and explicit attitudes were correlated with each other (at baseline), explicit attitudes were not associated with PA behavior at any measurement point as demonstrated in the regression models. Therefore, it is logical that explicit attitudes regarding PA prediction were unaffected by discrepancy. Also IED did not moderate the relationship between explicit attitude and intention. The null-findings could be a result of the fact that IED was not very strong in the sample, which is reflected in the low mean and can also be seen in the distribution of IED (Figure 3.2). This in turn could be due to the target behavior PA. The coherence between implicitly and explicitly measured attitudes regarding the same target is strong (as it was the case in the present study) when self-presentation concerns are weak and lower when self-presentation concerns are high (Hyde et al., 2010; Nosek, 2005; Nosek \& Banaji, 2016). Hence in the latter context, explicit attitudes are more likely to diverge from the unbiased (in terms of self-presentation) implicit attitudes. Karpen et al. (2012) investigated the effect of IED regarding alcohol consumption, which is a socially more sensitive topic and strongly influenced by social desirability (Davis, Thake, \& Vilhena, 2010). PA, on the other hand, is a much less socially sensitive topic, although also influenced by social desirability to some extent (Adams et al., 2005). Therefore, implicit and explicit attitudes in the context of alcohol consumption are more likely to deviate strongly from each other (e.g. implicitly being in favor of alcohol consumption but as a result of social desirability indicating it explicitly as negative) that assumingly created a stronger dissonance, which in turn had a stronger effect on the predictive power of explicit attitudes. It should be taken into account however, that the extent of the true experienced dissonance in the present study is unclear as it was inferred from the discrepancy between scores. Therefore, asking participants whether they experienced dissonance-based discomfort or negative emotions, as it has been done elsewhere (Maliszewski, 2011; Rydell et al., 2008), could be a valuable addition for future studies. Additionally, it should be noted that the evidence in support of the idea that social desirability results in IED is mixed (Berry, Rodgers, Markland, \& Hall, 2016; 
Hyde et al., 2010; Nosek, 2005). According to Gawronksi (2009) "the correspondence between implicit measures and self-reports is far more complex than just a matter of social desirability and self-presentation"(p. 144). Therefore, we encourage future studies to investigate possible other moderators of attitude congruence/dissonance, such as mindfulness (the ability to have insight into one's inner processes) which has been identified as moderator in other domains (Brown \& Cordon, 2009), but not yet in health psychology.

Further, a post-hoc analysis revealed that the true score correlation between the perceived pros and perceived cons scale was rather low (-.47), indicating that a response bias, such as tendency to agree, might have been present when participants answered the questions on explicit attitude. Although we reversed the scores for perceived pros before adding perceived cons, thereby correcting for tendency to agree to some extent, this bias was possibly present. This could be another reason for the present null-findings. Further research working with explicit attitude should ensure to offer a balanced number of positively and negatively worded questions, also for other relevant constructs involved, in order to prevent this potential bias. To assess whether the discrepancy between the explicit attitude and the implicit attitude was low and did not show the anticipated effects as the explicit attitude was comprised of instrumental and affective dimensions, we conducted post-hoc analyses. In these analyses we created a discrepancy index between the implicit attitude and the affective dimension of the explicit attitude only. It is assumed that implicit attitudes are rather grounded in affective associations, which is somewhat comparable to affective explicit attitudes. It could be reasonable that the addition of the instrumental dimension to the index distorted the discrepancy between the rather similar constructs and also its possible effect. Post-hoc analyses however did not reveal any differences regarding the results.

\section{Additional finding}

The study indicated that IED was negatively associated with baseline PA and PA behavior after one month. This finding applied to IED when it was comprised of the discrepancy between the instrumental and affective dimensions of the explicit attitude and the implicit attitude as well as when it was comprised of the discrepancy between the affective explicit attitude only and the implicit attitude. These findings are in line with studies demonstrating that IED affects (PA) behavior (Berry et al., 2018; Brand \& Antoniewicz, 2016; Brinol et al., 2006; Maliszewski, 2011; Petty et al., 2006; Rydell et al., 2008). The fact that IED was associated with PA behavior, even when explicit and implicit attitudes were not, is surprising but in accordance with the findings of Goldstein et al. (2014). IED predicted disinhibited eating while neither implicit nor explicit attitudes independently did. Contrary to Goldstein et al. (2014) who found a stronger tendency for disinhibited eating with increasing IED and argued that dissonance intensified the focus towards the object, we found a negative relationship. Thus IED in the context of PA is rather detrimental as it was negatively associated with PA levels. This could be due to the fact that a person holds inconsistent information about PA, i.e. liking PA (explicitly) but feeling (implicitly) less positive about PA (as indicated by the post-hoc analyses), which 
makes it harder to move towards the behavior. Therefore, our results suggest that, when aiming for more PA engagement, one should take the congruence of attitudes into account even when attitudes themselves are not directly related to the behavior. However, in order to draw a more generalizable conclusion, follow-up studies are highly encouraged, especially because the sample of the current study was very active. It is possible that results in a less active sample might differ. For example, a less active sample might hold less favorable/stable explicit attitudes towards PA, which might weaken the relationship between explicit attitude and intention and thereby IED might be more influenceable. Also it would be valuable to investigate whether the effect of IED loses its effect on the long-term. In the present study IED did not affect behavior after three months anymore. This could either be due to the fact that individuals manage to dissolve the dissonance over time leaving the behavior unaffected or due to the lower power at the follow-ups.

\section{Limitations}

When developing future studies, the following limitations of the study at hand could be taken into account. One limitation is that we only had students in our sample who on average had a quite positive explicit and implicit attitude towards PA, a high intention, as well as high activity levels. This is not representative for the general public (Teixeira et al., 2012) and could be another reason why dissonance was not as strong as expected. Therefore, future studies should make use of a sample with more varied attitudes towards PA. Also PA was measured by self-reports, which is likely to be inaccurate due to an over- or underestimation of activity levels (Prince et al., 2008). The low correlations between PA and well-known predictors of PA, i.e. self-efficacy, intention, indicate a rather inaccurate measurement of PA. Despite the demonstrated validity of the questionnaire we used (Wendel-Vos et al., 2003), it would be valuable to add objective measures such as accelerometers to provide an adequate report about PA levels (Prince et al., 2008; Slootmaker et al., 2009). Moreover, positive and negative words used for the SC-IAT were unrelated to PA. This is a common approach, which has been used in various former studies regarding PA or other behaviors (Friese et al., 2007; Goldstein et al., 2014; Hyde et al., 2010; Hyde et al., 2012; Karpinski \& Steinman, 2006; Rebar et al., 2015). It is possible, however, that implicit attitudes, and thereby IED, might have been different when negative or positive words related to PA (e.g. exhaustion or strength) were used. This would be in line with the assumption of the APE postulating that implicit attitudes are associative evaluations that "are best characterized as automatic affective reactions resulting from the particular associations that are activated automatically when one encounters a relevant stimulus" (Gawronski \& Bodenhausen, 2006, p. 693). This is an avenue for further research. In addition, discrepancy was inferred from the index. Whether discrepancy was experienced by the population is rather unclear. Questions about the experience of discrepancy or associated negative feelings could be a valuable addition for future studies. Another way to assess discrepancy could be to measure participants' automatic evaluations, share this information with them and let them rate eventual discrepancy between their reflective and automatic 
evaluation as it has been done by Brand et al. (2016). Also discrepancy was not manipulated, e.g. in an experimental setting. Therefore, causal inferences are rather hard to draw. In order to do so, follow-up studies with an experimental setting, such as performed in the study of Brand et al. (2016), would be valuable. Lastly, the test-retest correlation between the indices was rather low, especially between the index at baseline and the index after three months, indicating that the instrument is not stable over a longer period. It is unclear whether this is due to actual changes in IED, because of changes in measurement error due to time (also known as transient error), or an artefact of the substantial dropout over time.

\section{CONCLUSION}

We did not find explicit attitudes to be a weaker predictor for PA behavior or intention when IED was high. However, this finding cannot automatically be transferred to other health behaviors. Instead, since findings regarding the effect of IED on behavior are inconsistent, it can be concluded that the relationship between attitude, attitude discrepancy, behavior, and intention is more complex and probably also determined by other variables, such as the type of behavior or a person's ability to have insight into his or her implicit attitude or other inner processes (e.g. experiencing dissonance). Especially in the area of health psychology and health promotion, more research is needed in order to identify these behaviors and factors and how they interact with discrepant attitudes to eventually draw more generalizable conclusions. 

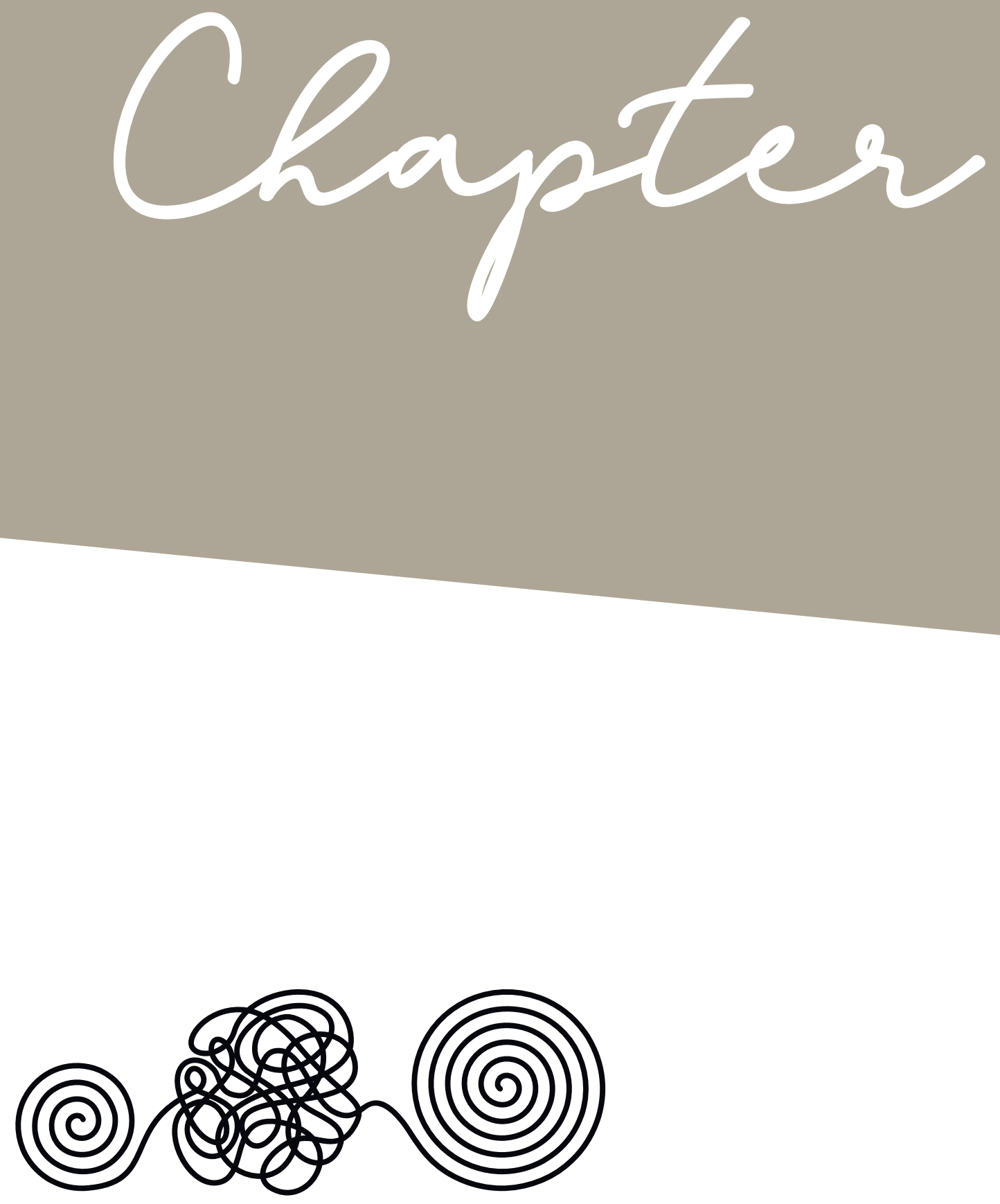


\section{fix:}

\section{Pathways of implicit attitudes, explicit attitudes, and behavior}

Muschalik, C., Candel, M.J.J.M., Crutzen, R., Elfeddali, I. \& de Vries, H. (under review). 


\section{A B S T R A C T}

Implicit attitudes and explicit attitudes predict behavior. Behavioral performance in turn, can feed back to a person's explicit attitude. It is unclear whether behavioral performance can also feed back to a person's implicit attitude. It is also unclear whether implicit attitudes and explicit attitudes influence each other as postulated in the Associative-Propositional Evaluation (APE) model. To address these questions, we analysed secondary data of two longitudinal studies regarding physical activity (PA) and red meat consumption (RMC), using structural equation modelling. Results showed that behavioral performance did not have an influence on implicit attitudes at a later point in time. Moreover, explicit attitudes did not influence implicit attitudes at a later point in time. For RMC, implicit attitudes did not have an influence on explicit attitude. For PA, however, implicit attitudes had a significant negative effect on perceived pros (explicit attitude). As almost all reciprocal relationships between implicit attitudes and explicit attitudes were non-significant, it is likely that they are rather unrelated constructs, which do not shape each other but are probably influenced by other factors. More studies are needed in order to check whether theoretical postulations put forward in the APE can be applied to real-life situations. 


\section{INTRODUCTION}

Explicit attitudes are conceptualized as a person's conscious view about concepts, objects, behaviors, or people, and are formed by underlying beliefs (Fishbein, 1963, 1967). Although beliefs are reasoned, this does not imply rationality. Beliefs, regardless of their rationality, form an individual's cognitive foundation for his or her responses to the world. It is assumed that only the beliefs that are accessible shape a person's explicit attitude (Fishbein, 1963). In case beliefs about a behavior are mostly positive (e.g. 'physical activity is healthy', 'physical activity is good for my body'), a person will report a positive explicit attitude towards the behavior, for instance in a questionnaire, and will be more likely to engage in the behavior. Another person whose beliefs are mostly negative (e.g. 'physical activity is uncool', 'physical activity is tiring') will express a negative explicit attitude towards the behavior. As a consequence, this person will be less likely to engage in the behavior at question. That is because explicit attitudes are, next to other explicit cognitions such as self-efficacy (i.e. the perceived ability to perform a behavior) and social norms (i.e. the perception of what other people think or do regarding a specific behavior), one of the most proximal factors for a person's intention to perform a certain behavior (Ajzen, 1991; De Vries, 2017; Fishbein \& Ajzen, 2011). Hence, the more positive a person's explicit attitude towards a behavior, the higher his or her intention to engage in the respective behavior. The contrary applies to negative explicit attitudes. Intention, in turn, is the most proximal determinant for behavioral performance. These relationships have been shown across a wide range of (health) behaviors, such as condom use, physical activity, meat consumption, pro-environmental behavior, and smoking behavior (Albarracin et al., 2001; Carfora et al., 2017a; Godin, Valois, Lepage, \& Desharnais, 1992; Graça et al., 2015; Hagger et al., 2002; Levine \& Strube, 2012; McEachan et al., 2011).

Also, a bidirectional relationship between explicit attitudes and behavior has been identified. That is, explicit attitudes have an influence on a person's behavior (for a review see: Greenwald et al., 2009; McEachan et al., 2016) and performing a behavior in turn feeds back to the beliefs that underlie the attitude, for instance new beliefs are added or existing beliefs are modified. This can be a result of cognitive dissonance reduction, e.g. a person becomes aware that he or she performed a behavior that contradicts his or her former attitude. A contradiction between a person's behavior and his or her attitude will likely result in dissonance, which is accompanied by negative feelings that the individual aims to resolve (Festinger, 1957). To do so, the counter-attitudinal behavior will be rationalized and the view about the behavior, thereby the attitude, will be adjusted (Festinger, 1957). Another mechanism is biased scanning, meaning that people conduct a biased search of their existing beliefs that confirm the legitimacy of the behavior they performed (Albarracín \& Wyer Jr, 2000; Janis \& King, 1954). It is assumed that biased scanning increases the salience of suitable beliefs and results in attitude change towards the behavior they performed. Independent of the underlying mechanism that led to a change in attitude after the behavioral performance, the "new" explicit attitude will again affect whether the person is more or less likely to pursue a behavior (Fishbein \& Ajzen, 2011). 
In the last decades, implicit attitudes have been studied extensively in health psychology. They are defined as "introspectively unidentified (or inaccurately identified) traces of past experience that mediate favorable or unfavorable feeling, thought, or action toward a social object" (p.8, Greenwald \& Banaji, 1995). Hence, individuals possess positive or negative implicit attitudes towards concepts, objects, behaviors, or people, without necessarily being aware of them. Contrary to explicit attitudes, people are not able to express their implicit attitudes in a conscious way. To assess them nonetheless, reaction time paradigms are used (e.g. Gawronski \& De Houwer, 2014; Karpinski \& Steinman, 2006). Although rather unconscious, implicit attitudes are also related to a person's behavior. For example, more positive implicit attitudes predicted higher activity levels (Conroy et al., 2010). In other studies, implicit attitudes predicted food choices (Friese et al., 2008; Richetin et al., 2007), voting behavior (Arcuri, Castelli, Galdi, Zogmaister, \& Amadori, 2008; Friese et al., 2007), alcohol consumption (Wiers et al., 2002), social interaction behavior (Allen McConnell \& Leibold, 2001), or the usage of brand products (Maison, Greenwald, \& Bruin, 2004).

The influence of both implicit and explicit attitudes on behavior is depicted in so-called dual-process models. The Reflexive-Impulsive Model (RIM; Strack \& Deutsch, 2004) or the Associative-Propositional Evaluation Model (APE; Gawronski \& Bodenhausen, 2006) are examples of dual-process models. Both models distinguish between a conscious or reflexive and an unconscious or impulsive system. Whereas the reflexive system entails reasoned, deliberate, and conscious motives to pursue a behavior, such as explicit attitudes, the impulsive system is comprised of automatic behavioral tendencies, such as implicit attitudes. The APE postulates that affective, hence, implicit reactions are used as a basis to make evaluative judgments, which are reported in explicit statements. This is assumed to be the case, when the implicit reaction is regarded as 'true' by the individual or when the reaction is consistent with other relevant beliefs. If, however, the implicit reaction is inconsistent with other relevant beliefs, consistency will be restored by, for instance, rejecting one of the involved beliefs or by looking for additional compatible beliefs (Gawronski \& Bodenhausen, 2006).

Dual-process models remain, however, silent about whether behavioral performance also feeds back to and influences an individual's implicit attitude, just as it is the case for explicit attitudes as mentioned above. To our knowledge, it is unclear whether implicit attitudes are shaped by a person's behavior, or in contrast act only as a precursor for behavior, or both. Previous research has indicated that implicit attitudes might be sensitive to the recent exposure or experience of the attitudinal object or behavior (Gawronski \& Bodenhausen, 2006; Rudman, 2004), but evidence is rather scarce. Hence, the first research question of this article is whether the performance of a behavior at one point in time influences a person's implicit attitude at a later point in time (RQ 1).

An additional question that has not been addressed so far is whether implicit attitudes and explicit attitudes also influence each other over time. More precisely, is it possible that a person's positive (or negative) implicit attitude towards an object at one point in time leads to 
a positive (or negative) explicit attitude towards the object at a later point in time? According to Gawronski and Bodenhausen and their assumptions put forward in the APE (2006), automatic affective reactions toward an object, hence implicit attitudes, are by default used as a basis for evaluative judgments about this object, hence explicit attitudes. More precisely, the individual decides whether his or her implicit attitude toward an object is valid (for him or her) and whether it is used as a default for his or her explicit attitude toward the object or not. Hence, explicit attitudes can be dependent of implicit attitudes when latter ones are regarded as a valid basis, but they can also be independent of implicit attitudes, namely when the latter construct is regarded as invalid. Until now, it is unclear in how far implicit attitudes influence a person's explicit attitude. Moreover, implicit attitudes towards different behaviors were shown to change naturally over a period of one week (Hyde et al., 2012) as well as over a period of two months (Gawronski et al., 2017). According to Gawronski et al. (2017) these changes "may generalize to explicit measures to the extent that the newly created associations are regarded as valid" (p. 301). It is unclear whether this is the case. Therefore, the second research question of this article is whether a person's implicit attitude has an influence on a person's explicit attitude at a later point in time (RQ 2).

Finally, Gawronski and Bodenhausen (2006) postulated that this process can be applied in the other direction as well, hence a change in explicit attitude might lead to a change in implicit attitude. More precisely, newly acquired information regarding a specific behavior (e.g. 'physical activity is an effective way to combat non-communicable diseases') will be reflected in a person's explicit attitude. This process, in turn, might lead to the formation of new associations between the object (e.g. physical activity) and its evaluation (e.g. positive). The third research question in this article, therefore, is whether a person's explicit attitude at one point in time influences a person's implicit attitude at a later point in time (RQ 3).

\section{METHODS}

The current study used secondary data from two previous studies. The first dataset consists of variables representing explicit cognitions (explicit attitude comprised of perceived pros and perceived cons, social norms, self-efficacy) towards red meat consumption (RMC), selfreported consumption of red meat and implicit attitude towards red meat (Chapter 3 ). The second dataset consists of the same explicit cognitions, however, regarding physical activity (PA). Also, self-reported activity levels and implicit attitudes towards PA were administered (Muschalik et al., 2018). Both studies consisted of a baseline measurement and follow-ups after one and three months thereafter. The assessment of the implicit attitude was followed by the assessment of the explicit cognitions. 


\section{Dataset 1}

\section{Participants and procedure}

Dataset 1 consists of data from a sample of the Dutch population. At baseline, data from 1476 individuals were available ( $47 \%$ female, mean age $=49, \mathrm{SD}=15.90)$. After one month, data from 708 individuals were available (48\% of baseline, $47 \%$ female, mean age $=48$, SD $=15.18$ ) and after three months, data from 467 participants were present (32\% of baseline, $44 \%$ female, mean age $=50, S D=15.67$ ). The study was administered online and participants were recruited by two internet research agencies. The full procedure of the study is described elsewhere (Chapter 3).

\section{Measurements}

Explicit cognitions towards red meat consumption

The questions to assess explicit cognitions were based on the I-change model (De Vries, 2017). In the model, explicit attitudes are operationalized as perceived pros and perceived cons of a behavior. Hence we assessed the explicit cognitions explicit attitude, comprised of ten perceived pros, ten perceived cons regarding red meat consumption, four social norm questions, four social modelling questions, and nine self-efficacy beliefs regarding reduced red meat consumption. One example for pros is "Eating red meat is" (1) "not tasty" to (5) "very tasty", expressed on a 5-point Likert scale. Also on a 5-point Likert scale, norms of family members, partners, and friends regarding reducing red meat consumption were assessed as well as their behavior. A norm item was "Most members of my family" (1) "don't think that I have to reduce my red meat intake" to (5) "certainly think that I have to reduce my red meat intake". A modeling item was "How many of your family members consume red meat?" with answers ranging from (1) "None of them" to (5) "All of them". Self-efficacy was assessed by nine items and was based on perceived barriers to reduce one's meat intake (Dibb \& Fitzpatrick, 2014). These items asked participants on a 5-point Likert scale to indicate to what extent they perceive themselves as capable of reducing their red meat intake, for example "I will be able to reduce my red meat consumption even when I am used to eat red meat" with answer options from (1) "completely disagree" to (5) "completely agree". The full questionnaire is available at https://mfr.osf.io/render?url=https://osf.io/xwgpz/?action=download\%26mode=render. For each explicit cognition, four items with the highest factor loadings were selected and used in the analyses (perceived pros: $\alpha=.77$; perceived cons: $\alpha=.70$; social norms: $\alpha=.77$; social modeling: $\alpha=.58$; self-efficacy: $\alpha=.84$ ).

\section{Red meat consumption}

Levels of red meat consumption were assessed by means of two items, which is based on the Food Frequency Questionnaire. The first item asked on how many days per week the person consumes red meat (1-7 days per week; with the additional answer option 'Not on a daily basis 
but at least once a month'). The second item asked how many grams the person consumes on these days (open question). A reference point was added as an example (e.g. a piece of prepared meat at the main meal equals $100 \mathrm{gr}$ and a slice of meat topping (e.g. ham) equals $15 \mathrm{gr})$. The weekly amount of consumed red meat was calculated by multiplying the frequency per week by the average daily amount of grams.

Implicit attitudes towards red meat

A modified Single-Category Implicit Association Task (SC-IAT; Karpinski \& Steinman, 2006) was used to assess implicit attitudes towards red meat. Pictures were used to represent red meat and positive and negative words as evaluative stimuli. All stimuli were pretested and the SC-IAT was programmed in Inquisit (version 4). The improved scoring algorithm was used to calculate d-scores (Greenwald et al., 2003). D-scores are expressed in milliseconds and represent the strength of an association between concepts as measured by the standardized mean difference score of 'hypothesis-inconsistent' pairings and 'hypothesis-consistent' pairings of the stimuli (Greenwald et al., 2003). Scores ranged from - 1 to 1 and a higher score indicated a more positive implicit attitude towards red meat. A more detailed description of the SC-IAT that was used in the study can be found elsewhere (Chapter 3).

\section{Additional variables}

Additionally, participants' age, gender, and level of education (categorized into low, medium, and high according to the Dutch educational system) were assessed.

\section{Dataset 2}

\section{Participants and procedure}

Dataset 2 consists of data from students of a Dutch University. At baseline, 340 students ( $61 \%$ female, mean age $=21, S D=2.11)$ participated. One month later, 240 students filled out the same measures ( $71 \%$ of baseline, $64 \%$ female, mean age $=21, \mathrm{SD}=2.14$ ) and three months later a total of 128 students ( $38 \%$ of baseline, $69 \%$ female, mean age $=22, \mathrm{SD}=2.19$ ) completed the measures. The data were collected in a lab. The detailed procedure of the study is described elsewhere (Muschalik et al., 2018).

\section{Measurements}

\section{Explicit cognitions towards physical activity}

Explicit cognitions including perceived pros, perceived cons, social norms, social modeling, and self-efficacy regarding physical activity were assessed by means of a questionnaire. The questions were again based on the I-change model (De Vries, 2017). One example for a pro item is "When I am adequately active it is" (1) "not good for my health" to (5) "very good for my health", expressed on a 5-point Likert scale. Social norms and social modeling were each 
assessed by four questions. On a 5-point Likert scale the norms about adequate physical activity of family members, partners, and friends as well as their PA behavior were assessed. A norm item was "Most of my friends" (1) "certainly think that I need to be adequately active" to (5) "certainly do not think that I should be adequately active". A modeling item was "Most of my friends are adequately physically active" with answers possible from (1) "totally agree" to (5) "totally disagree". Self-efficacy was measured by nine items, also on a 5- point Likert scale, asking to what extent respondents thought they would be able to be adequately physically active in different situations. For instance, "I find it difficult/easy to be adequately physically active when I am tired" with answer options from (1) "very difficult" to (5) "very easy". Where appropriate, items were reversed so that higher values represent stronger social modeling, social norms, or self-efficacy. For the full questionnaire, see https://doi.org/10.1186/s40359018-0229-0. For each explicit construct, four items with the highest factor loadings were selected and used in the analyses (perceived pros: $\alpha=.79$; perceived cons: $\alpha=.74$; social norms: $\alpha=.60$; social modeling: $\alpha=.40$; self-efficacy: $\alpha=.75$ ).

\section{Physical activity levels}

Physical activity levels were assessed by means of the Short Questionnaire to Assess Healthenhancing physical activity (SQUASH), a reliable and valid tool to assess activity levels among a Dutch adult population (Wendel-Vos et al., 2003). A more detailed description of the SQUASH and how activity levels were calculated can be found elsewhere (Muschalik et al., 2018).

\section{Implicit attitudes towards physical activity}

Implicit attitude towards physical activity was assessed by means of a modified SC-IAT (Karpinski \& Steinman, 2006). Words representative for physical activity and negative and positive words were used as evaluative stimuli. Stimuli for the SC-IAT were pretested and Inquisit (Version 4) was used to program the SC-IAT. Implicit attitudes were represented by means of d-scores, using the improved scoring algorithm (Greenwald et al., 2003). A detailed description of the SC-IAT that was used in the study can be found elsewhere (Muschalik et al., 2018). Scores ranged from -1 to 1 and a higher score indicated a more positive implicit attitude towards physical activity.

\section{Additional variables}

Additionally, participants' age, gender, and level of education categorized into low, medium, and high (according to the Dutch educational system) were assessed.

\section{Analyses}

Structural Equation Modelling using MPlus Version 7.2 (Muthén \& Muthén, 2012) was used to test reciprocal relationships between implicit attitudes, perceived pros and perceived cons, and behavior over time. For both datasets the full conditional specification method (with 
the sequential regression procedure) was used to impute missing data. Several simulation studies suggest that this imputation method produces unbiased parameter estimates and standard errors (Raghunathan, Lepkowski, Van Hoewyk, \& Solenberger, 2001; Van Buuren, Brand, Groothuis-Oudshoorn, \& Rubin, 2006). The number of imputations was 73 for the RMC dataset and was 75 for the PA dataset, the number of imputations being at least as large as the percentage of incomplete cases (White, Royston, \& Wood, 2011). The explicit constructs perceived pros, perceived cons, self-efficacy, social norms, and social modeling were shown to influence each other (e.g. Hagger et al., 2002). To control for these influences, these explicit cognitions were included in all analyses. For perceived pros, perceived cons, self-efficacy, social norms, and social modeling item scores were averaged and used as scores for these constructs. Next to the implicit attitude and behavior, also the variables age, gender, and level of education were added to correct for possible confounding. The model fit was estimated by means of the Root Mean Square Error of Approximation (RMSEA) and the comparative fit index (CFI). A good model fit is indicated by a low RMSEA (<0.08) and a high CFI (>0.9) (Osterlind, Tabachnick, \& Fidell, 2001). The estimated models for both datasets indicated a good model fit (Red meat consumption: mean RMSEA $=0.04$, mean CFI $=0.97$; Physical activity: mean RMSEA $=0.00$, mean CFI = 1.00).

\section{RE S U LTS}

\section{Descriptives}

Descriptives of both samples (of complete cases) are depicted in table 6.1 (for RMC) and table 6.2 (for PA). In the sample regarding RMC, individuals ate on average 470 grams of red meat per week and reported some perceived pros of RMC $(M=3.53$ on a scale from 1-5) and a few perceived cons ( $M=2.04$ on a scale from 1-5). The implicit attitude towards red meat was slightly negative $(M=-.02)$. In the PA sample, participants reported many perceived pros of PA $(M=4.33$ on a scale from 1-5) and a few perceived cons of PA ( $M=2.38$ on a scale from 1-5). The implicit attitude towards PA was slightly positive $(M=.06)$.

Table 6.1 | RMC: Characteristics of study sample and differences over time (for complete cases)

\begin{tabular}{|c|c|c|c|c|c|c|}
\hline & $\begin{array}{c}\text { T0 } \\
(\mathrm{N}=398)\end{array}$ & $\begin{array}{c}\text { T1 } \\
(n=398)\end{array}$ & $\begin{array}{c}\text { T2 } \\
(n=398)\end{array}$ & $\mathbf{F}$ & df & p \\
\hline Age (mean, SD) & $50(15.64)$ & $-*$ & $-*$ & $-*$ & $-*$ & $-*$ \\
\hline Gender (female), n (\%) & 178 (45\%) & $-*$ & $-*$ & $-*$ & $-*$ & $-*$ \\
\hline Perceived Pros (mean, SD) & $3.53(.64)$ & $3.56(.63)$ & $3.76(.63)$ & 15.22 & 2 & $<.001$ \\
\hline Perceived Cons (mean, SD) & $2.04(.65)$ & $2.07(.63)$ & $2.09(.64)$ & .85 & 2 & .43 \\
\hline Implicit attitude (mean, SD) & $-.02(.31)$ & $-.04(.34)$ & $-.06(.31)$ & .96 & 2 & .38 \\
\hline $\begin{array}{l}\text { Red meat consumption (gr/week) } \\
\text { (mean, SD) }\end{array}$ & $470.14(335.69)$ & $503.66(355.86)$ & $486.27(348.16)$ & .93 & 2 & .40 \\
\hline
\end{tabular}

*are stable over time and are therefore not named again nor tested for differences 
Table 6.2 | PA: Characteristics of study sample and differences over time (for complete cases)

\begin{tabular}{|c|c|c|c|c|c|c|}
\hline & $\begin{array}{c}\text { T0 } \\
(\mathrm{N}=120)\end{array}$ & $\begin{array}{c}\text { T1 } \\
(n=120)\end{array}$ & $\begin{array}{c}\text { T2 } \\
(n=120)\end{array}$ & $\mathrm{F}$ & df & $\mathrm{p}$ \\
\hline Age (mean, SD) & $21(2.13)$ & $-*$ & $-*$ & $-*$ & $-*$ & $-*$ \\
\hline Gender (female), n (\%) & 207 (61\%) & $-*$ & $-*$ & $-*$ & $-*$ & $-*$ \\
\hline Perceived Pros (mean, SD) & $4.33(.53)$ & $4.26(.56)$ & $4.22(.57)$ & 1.27 & 2 & .28 \\
\hline Perceived Cons (mean, SD) & $2.38(.71)$ & $2.17(.71)$ & $2.19(.69)$ & 3.37 & 2 & .04 \\
\hline Implicit attitude (mean, SD) & $.06(.30)$ & $.10(.34)$ & $.15(.33)$ & 2.03 & 2 & .13 \\
\hline Physical activity score (mean, SD) & $4868.59(3030.29)$ & $5364.23(.2955 .11)$ & $5677.46(2845.22)$ & 2.30 & 2 & .10 \\
\hline
\end{tabular}

*are stable over time and are therefore not named again nor tested for differences

Does behavior influence a person's implicit attitude at a later point in time (RQ 1)?

RMC at baseline did not have a significant effect on implicit attitude at T1 ( $\beta=-.02 ; p=.71$ ). Also, RMC at T1 did not have a significant effect on implicit attitude at $\mathrm{T} 2(\beta=-.002 ; p=.98)$. For a full model depicting the significant pathways, see figure 6.1 .

$\mathrm{PA}$ at baseline did not have a significant effect on implicit attitudes at $\mathrm{T} 1 \quad(\beta=.04 ; p=.52)$, nor did PA at T1 have a significant effect on implicit attitudes at T2 $(\beta=-.02 ; p=.85)$. For the full model, see figure 6.2. In sum, we did not find that behavior at one point in time influenced a person's implicit attitude at a later point in time.

Does the implicit attitude influence a person's explicit attitude at a later point in time (RQ2)?

In the context of RMC, implicit attitude at baseline did not have a significant effect on perceived $\operatorname{pros}(\beta=-.03 ; p=.18)$ or perceived cons at $\mathrm{T} 1(\beta=-.03 ; \mathrm{p}=.16)$. Also, implicit attitude at T1 did not have a significant relationship with perceived pros $(\beta=.03 ; p=.28)$ or perceived cons at $\mathrm{T} 2(\beta=-.04 ; p=.26)$.

Regarding PA, implicit attitude at baseline had a significant negative effect on perceived pros at $\mathrm{T} 1(\beta=-.08 ; \mathrm{p}=.05)$ but not on perceived cons at $\mathrm{T} 1(\beta=.02 ; \mathrm{p}=.58)$. Implicit attitude at $\mathrm{T} 1 \mathrm{did}$ not have a significant relationship with perceived pros at $\mathrm{T} 2(\beta=.11 ; \mathrm{p}=.07)$ or perceived cons at $\mathrm{T} 2(\beta=-.03 ; p=.61)$.

Does a person's explicit attitude at one point in time influence a person's implicit attitude at a later point in time (RQ3)?

In the context of RMC, neither perceived pros $(\beta=.07 ; p=.16)$ nor perceived cons at baseline ( $\beta=-.06 ; p=.22$ ) had a significant effect on implicit attitude at T1. Perceived cons at baseline had a significant negative effect on perceived pros at T1 $(\beta=-.13 ; p<.01)$. No significant relationship between perceived pros $(\beta=.11 ; p=.06)$ and perceived cons at $\mathrm{T} 1$ ( $\beta=-.01 ; \mathrm{p}$ $=.93$ ) and implicit attitude at T2 was found. Again, perceived cons at T1 had a significant negative relationship with perceived pros at $\mathrm{T} 2(\beta=-.08 ; \mathrm{p}=.03)$. 
Concerning PA, neither perceived pros $(\beta=.06 ; p=.30)$ nor perceived cons $(\beta=.00 ; p=.95)$ at baseline had an influence on implicit attitudes at T1. Also at T1, perceived pros $(\beta=.05 ; p=.66)$ and perceived cons ( $\beta=-.07 ; p=.54)$ did not have an effect on implicit attitudes on T2. In sum, a person's explicit attitude (perceived pros or perceive cons) at one point in time did not influence a person's implicit attitude at a later point in time. 


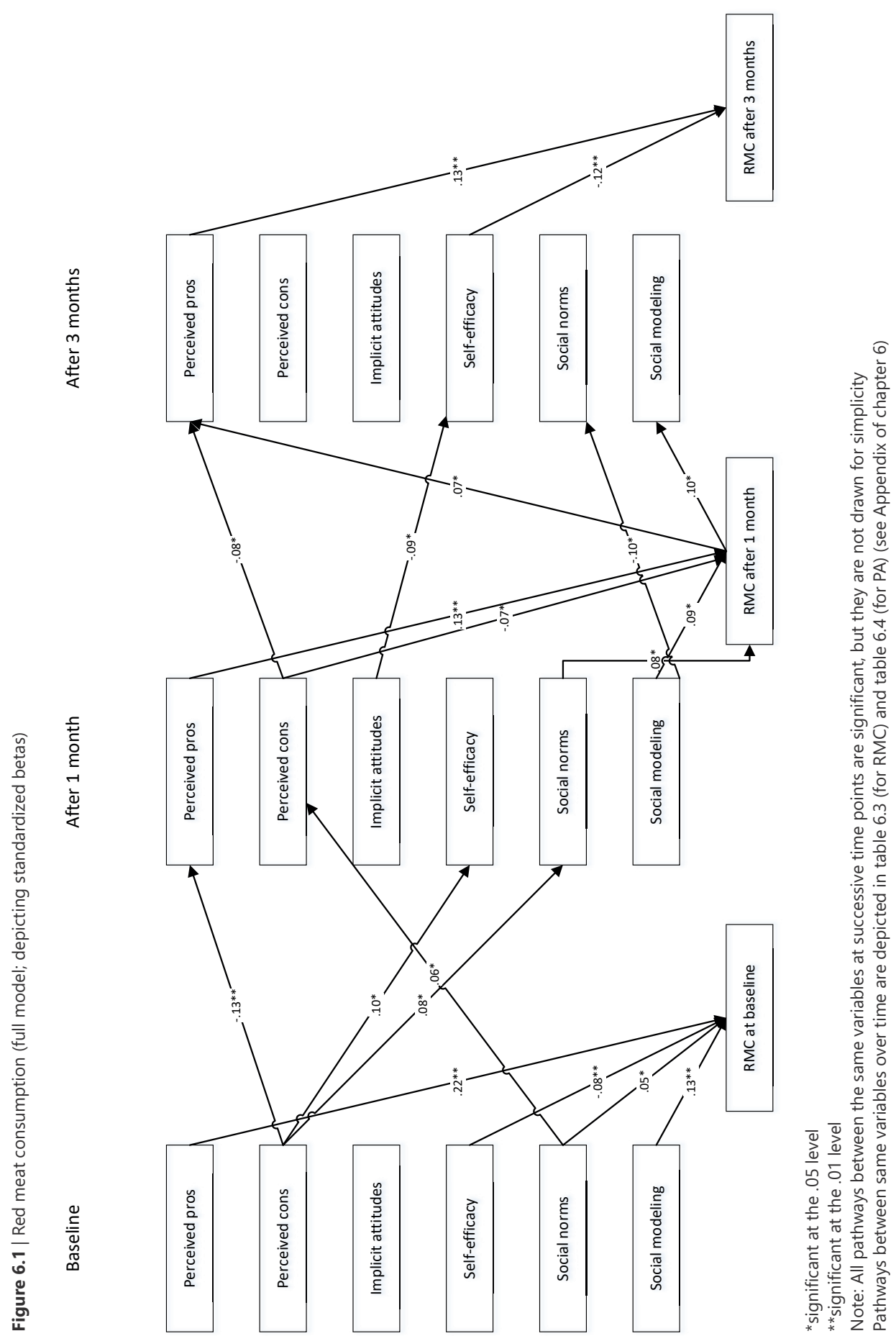




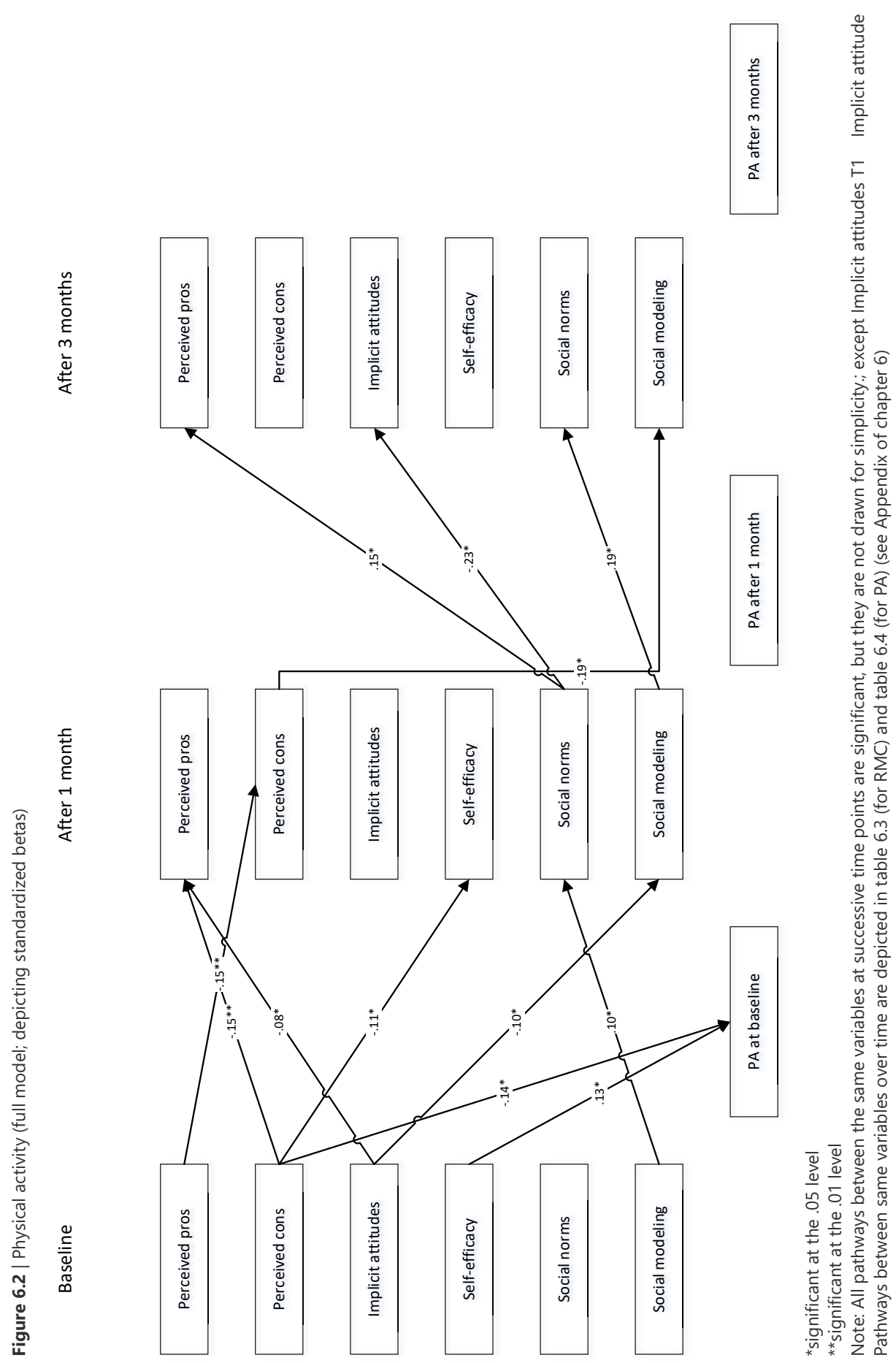




\section{DISCUSSION}

In this study, we investigated whether implicit attitudes, explicit attitudes, and behavior (RMC or PA) influence each other over time. To our knowledge, this is the first study to analyze these relationships over a period of three months.

Neither the behavioral performance of RMC nor of PA had an influence on a person's implicit attitude at a later point in time (RQ 1). What we found for RMC, however, was that behavioral performance at baseline resulted in more perceived pros of RMC after one month. This pattern was already known before (e.g. Albarracín \& Wyer Jr, 2000; Fishbein \& Ajzen, 2011) and can be explained by the cognitive dissonance reduction (Festinger, 1957) or by biased scanning (Albarracín \& Wyer Jr, 2000; Janis \& King, 1954) described above. In short, a person adjusts its former attitude or conducts a biased search of beliefs to confirm the legitimacy of the performed behavior. It is possible that this is not valid for implicit attitudes since implicit attitudes are rather unconscious attitudes, which might be difficult to become aware of and thereby hard to adjust consciously. A change in implicit attitude is more likely to be expected by means of evaluative conditioning (Ebert, Steffens, von Stülpnagel, \& Jelenec, 2009; Hollands et al., 2011; Mattavelli, Avishai, Perugini, Richetin, \& Sheeran, 2017), e.g. the experience of the behavior would have to be paired with something positive or negative. As we did not make use of an experimental setting, it is unclear with what kind of experience the behavior was paired or whether it was paired with a positive or negative experience at all. PA, as well as RMC, are likely to be habitual behaviors, which people perform according to their individual long-established patterns. Therefore, it is possible that people did not necessarily have new experiences with these behaviors. This might explain the non-significant relationship between behavioral performance and a person's implicit attitude at a later point in time.

Regarding RMC, we did not find any reciprocal relationships between implicit attitudes and explicit attitudes. More precisely, implicit attitudes did not influence explicit attitudes at a later point in time (RQ 2). This might indicate that implicit attitudes were not used as a basis to form one's explicit attitude as they were considered as invalid, i.e. when they are dissonant with other relevant propositions (Gawronski \& Bodenhausen, 2006). For example, a positive implicit attitude towards RMC (e.g. 'I like to consume red meat') may be inconsistent with an explicit proposition (e.g. 'It is ethically not okay to eat meat'). As suggested in the APE (Gawronski \& Bodenhausen, 2006), this inconsistency might result in the rejection of the implicit attitude as a valid basis for one's explicit attitude. Moreover, implicit attitudes towards different behaviors were shown to change naturally over a period of one week or two months (Gawronski et al., 2017; Hyde et al., 2012). This was, however, not the case in the present study, in which implicit attitudes did not change over time $(p=.38)$. Hence, it is logical that explicit attitudes remained unaffected. In other studies, implicit attitudes were changed actively, e.g. by means of evaluative conditioning or persuasion, which then resulted in corresponding changes in the explicit attitude (Gawronski \& Bodenhausen, 2006). However, the present study made use of an observational design rather than of an experimental design. Also, explicit attitudes 
did not influence a person's implicit attitude at a later point (RQ 3). Perceived pros at T2 were significantly higher at $\mathrm{T} 2$ than at baseline or at $\mathrm{T} 1$. It is possible that this increase might have affected implicit attitudes at a later point in time (Gawronski \& Bodenhausen, 2006). This was, however, not assessed in the current study. The null-findings regarding the relationships between implicit attitudes and explicit attitudes might indicate that they are independent constructs, which do not have an influence on each other. This postulation has been concluded from former studies and was based on the fact that implicit and explicit attitudes were mostly weakly correlated with each other (e.g. Blair, 2001; Hofmann, Gschwendner, Nosek, et al., 2005; Nosek, 2005). As other authors had opposing conclusions, e.g. that implicit attitudes and explicit attitudes are indeed related to each other (e.g. Gschwendner, Hofmann, \& Schmitt, 2006; Hofmann, Gschwendner, Nosek, et al., 2005), we encourage future research to conduct replication studies of the present study in order to draw more generalizable conclusions.

Also, for PA, explicit attitudes did not have an effect on implicit attitudes (RQ 3), which could also be explained by the aforementioned reasoning. What we did find for PA, however, is an effect of implicit attitudes on explicit attitudes (RQ 2). That is, the more positive the implicit attitude towards PA at baseline, the lower the perceived pros of PA at T1. Although this might seem surprising in the first place, this could be explained by means of the APE (Gawronski $\&$ Bodenhausen, 2006). That is, the positive implicit evaluation of PA might not have been considered as valid, when forming one part of one's explicit attitude -perceived pros of PA. This could be due to the fact that more perceived cons at baseline were also negatively related to perceived pros at T1. As perceived cons might be relevant propositions but are inconsistent with the implicit attitude, it is possible that the implicit attitude was considered as invalid when forming one part of one's explicit attitude i.e. perceived pros. This would be in line with the APE (Gawronski \& Bodenhausen, 2006), according to which "inconsistency among [...] propositions may lead to a rejection of the [...] affective reaction as a valid basis for an evaluative judgment" (Gawronski \& Bodenhausen, 2006, p. 694).

\section{Limitations}

When interpreting the current findings, the following limitations should be taken into account. First, both behaviors were measured by means of self-reports. Although we used validated tools (SQUASH for PA (Wendel-Vos et al., 2003)) or built our measure on validated tools (FFQ for RMC), self-report measures are prone to reporting errors, such as over- or underestimation (Adams et al., 2005; Durante \& Ainsworth, 1996; Hebert, Clemow, Pbert, Ockene, \& Ockene, 1995; Hebert et al., 1997). Implicit attitudes have been shown to be related to behavior especially when it was measured objectively (e.g. Conroy et al., 2010; Friese et al., 2007; Maison et al., 2004). Possibly, we did not find a relationship between previous behavioral performance and later implicit attitudes due to the subjective measurements used, thereby resulting in distorted relationships between both constructs. Before being able to conclude whether behavioral performance does or does not feedback to implicit attitudes, we encourage future studies to measure behavior more objectively, e.g. activity levels by means of pedometers. RMC on 
the other side appears much more difficult to be measured objectively. Taking pictures or weighting meals before consuming them might offer alternatives, however, these measures are again dependent on the individual and, therefore, prone to reporting errors, e.g. forgetting to take pictures, or not weighting correctly. Second, we measured explicit attitude (perceived pros and perceived cons) regarding red meat consumption whereas implicit attitudes were measured regarding red meat. This was mainly due to the fact that the behavior red meat consumption was rather difficult to clearly depict on stimuli. Therefore, we used the approach also used in former studies, which also had to deal with this issue (Huijding et al., 2005; Waters et al., 2007), and measured the implicit attitude towards the target (red meat) and the explicit attitude towards the behavior (red meat consumption). It is possible that this approach led to a weaker relationship between the two types of attitudes regarding RMC. Alternatively, it is possible that these two types of attitudes do not influence each other over time as this was also the case for implicit and explicit attitudes regarding PA, which were measured towards the same object. Third, due to many missing cases in the dataset of PA at the second and third measurement, we performed multiple imputation - a valid simulation-based statistical technique for handling missing data (Jakobsen, Gluud, Wetterslev, \& Winkel, 2017) - in order to have sufficient power for the analyses. However, the percentage of incomplete cases was $65 \%$ for PA and $73 \%$ for RMC and might have been too large (Jakobsen et al., 2017) to create reliable data. Whether the relationships between the two types of attitude and behavior would have been different for other data is currently unclear and should be investigated by future studies.

\section{CONCLUSION}

Across two different types of behaviors (RMC and PA), almost all reciprocal relationships between implicit attitudes, explicit attitudes, and behavior were non-significant. This indicates that implicit attitudes and explicit attitudes are rather unrelated constructs, which do not shape each other but are probably influenced by other factors (e.g. underlying beliefs, learned associations, self-presentation concerns etc.). Further studies are needed in order to check whether theoretical postulations put forward in the APE (i.e. whether a change in a person's implicit attitude can result in a change of the person's explicit attitude or vice versa; Gawronski \& Bodenhausen, 2006) can be applied to real-life situations and samples. 


\section{Appendix}

Table 6.3. Pathways between same variables over time for RMC.

\begin{tabular}{lll}
\hline Paths & $\beta$ & $\mathrm{p}$ \\
\hline Perceived pros T0 $\rightarrow$ Perceived pros T1 & .68 & $<.001$ \\
Perceived pros T1 $\rightarrow$ Perceived pros T2 & .51 & $<.001$ \\
Perceived cons T0 $\rightarrow$ Perceived cons T1 & .73 & $<.001$ \\
Perceived cons T1 $\rightarrow$ Perceived cons T2 & .50 & $<.001$ \\
Implicit attitude T0 $\rightarrow$ Implicit attitude T1 & .17 & $<.001$ \\
Implicit attitude T1 $\rightarrow$ Implicit attitude T2 & .16 & $<.001$ \\
RMC T0 $\rightarrow$ RMC T1 & .56 & $<.001$ \\
RMC T1 $\rightarrow$ RMC T2 & .34 & $<.001$ \\
Social modeling T0 $\rightarrow$ Social modeling T1 & .65 & $<.001$ \\
Social modeling T1 $\rightarrow$ Social modeling T2 & .41 & $<.001$ \\
Social norms T0 $\rightarrow$ Social norms T1 & .52 & $<.001$ \\
Social norms T1 $\rightarrow$ Social norms T2 & .40 & $<.001$ \\
Self-efficacy T0 $\rightarrow$ Self-efficacy T1 & .41 & $<.001$ \\
Self-efficacy T1 $\rightarrow$ Self-efficacy T2 & .36 & $<.001$ \\
\hline
\end{tabular}

Table 6.4 Pathways between same variables over time for PA.

\begin{tabular}{llr}
\hline Paths & $\beta$ & $\mathrm{p}$ \\
\hline Perceived pros T0 $\rightarrow$ Perceived pros T1 & .69 & $<.001$ \\
Perceived pros T1 $\rightarrow$ Perceived pros T2 & .37 & $<.001$ \\
Perceived cons T0 $\rightarrow$ Perceived cons T1 & .64 & $<.001$ \\
Perceived cons T1 $\rightarrow$ Perceived cons T2 & .31 & .01 \\
Implicit attitude T0 $\rightarrow$ Implicit attitude T1 & .41 & $<.001$ \\
Implicit attitude T1 $\rightarrow$ Implicit attitude T2 & .12 & .19 \\
PA T0 $\rightarrow$ PA T1 & .61 & $<.001$ \\
PA T1 $\rightarrow$ PA T2 & .41 & $<.001$ \\
Social modeling T0 $\rightarrow$ Social modeling T1 & .65 & $<.001$ \\
Social modeling T1 $\rightarrow$ Social modeling T2 & .35 & .002 \\
Social norms T0 $\rightarrow$ Social norms T1 & .66 & $<.001$ \\
Social norms T1 $\rightarrow$ Social norms T2 & .36 & .002 \\
Self-efficacy T0 $\rightarrow$ Self-efficacy T1 & .69 & $<.001$ \\
Self-efficacy T1 $\rightarrow$ Self-efficacy T2 & .33 & .003 \\
\hline
\end{tabular}


Chapter 


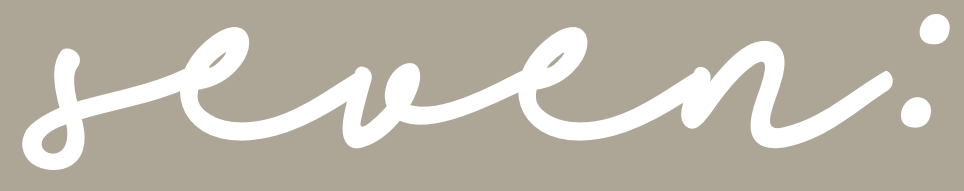

General Discussion 
The overall aim of this doctoral thesis is to describe how both explicit and implicit cognitions mutually predict behavior as well as how these two types of cognitions relate to each other. Until now, many interventions mostly focus on changing explicit cognitions, thereby often neglecting the rather unconscious, automatic drivers of behavior. This could be one possible reason why many behavior change interventions show modest success (Conn et al., 2011; Webb, Joseph, Yardley, \& Michie, 2010). Dual-process approaches integrate explicit and implicit cognitions in the prediction of behavior (e.g. Gawronski \& Bodenhausen, 2006; Strack \& Deutsch, 2004); implicit cognitions are often referred to or interpreted as implicit attitudes and explicit cognitions as explicit attitudes. However, other explicit cognitions, such as self-efficacy or social norms, are well-known to also predict behavior (e.g. De Vries, 2017; Fishbein \& Ajzen, 2011). As a result, different studies have been conducted to investigate when and under which circumstances implicit attitudes or explicit attitudes predict behavior best (Dovidio, Kawakami, Johnson, Johnson, \& Howard, 1997; Friese et al., 2008; Friese, Wänke, \& Plessner, 2006; Hofmann, Friese, \& Roefs, 2009; Hofmann et al., 2007; Nosek, 2005), but not how implicit attitudes jointly influence behavior with other explicit cognitions besides explicit attitudes. Although different patterns were suggested (Perugini, 2005), it is unclear whether one and the same pattern(s) apply to various behaviors or whether this is behavior dependent. To our knowledge, only one model presently exists that proposes to integrate implicit attitudes with explicit cognitions, such as self-efficacy and intention (Hagger \& Chatzisarantis, 2014); however, this model has an exclusive focus on physical activity and is not tested. According to this model, implicit attitudes have a direct influence on behavior. Other possible ways of influence, i.e. interactive patterns, were not included in the model nor tested. Hence, it remains unclear whether this model can be applied to other health behaviors or not as well as whether other patterns exist.

To address this gap, we looked at two suggested ways on how explicit cognitions and implicit attitudes could jointly guide behavior- the interactive pattern and the additive pattern (Perugini, 2005) - across two health behaviors: physical activity (PA) and red meat consumption (RMC). Moreover, we applied these two patterns to the prediction of intention, which has not been investigated before. Although intention does not always result in actual behavioral performance (Armitage \& Conner, 2001; Sheeran, 2002), it is one of the most proximal determinants for behavior. A better understanding of intention formation may help in the endeavor to change behavior. Moreover, we investigated the effects of implicitexplicit discrepancy (IED) on intention and behavior and whether the construct mindfulness is associated with less IED as well as whether mindfulness moderates the effects of IED on intention and behavior. Furthermore, the pathways of implicit attitudes, explicit attitudes, and behavior were analyzed, in order to understand if and how these constructs influence each other over time as well as to indicate where implicit attitudes could/should be integrated into a model.

For behavior prediction, we found support for the interactive pattern (Chapter 2 and 3) for PA and RMC. The relationship between self-efficacy and behavior was moderated by implicit 
attitudes in both studies. More precisely, perceiving oneself as capable of being able to perform a specific behavior had a stronger impact on the behavior when the implicit attitude towards the behavior/object was aligned with the person's self-efficacy, e.g. high self-efficacy to be physically active and a positive implicit attitude towards PA, high self-efficacy to be able to reduce RMC and a negative implicit attitude towards RM.

For intention prediction, support for the additive pattern (for RMC) and for the interactive pattern (for PA and RMC) was found. The additive pattern was only present in the context of $\mathrm{RMC}$, in which positive implicit attitudes towards RM were associated with a lower intention to reduce one's RMC. Until now only a few other studies have investigated the relationship between implicit attitude and intention and found implicit attitudes indeed to be associated with the intention to perform a specific behavior (Berry et al., 2011; Brochu \& Morrison, 2007; Dal Cin et al., 2007; Dasgupta \& Rivera, 2008). However, as far as we know studies regarding the association between implicit attitudes and intention remain scarce. Based on our finding and those of others, we conclude that implicit attitudes are not solely related to behavior but also to intention. Hence, modifying implicit attitudes might not only result in a change of behavior but also in a change of one of its most proximal determinant, namely intention. Therefore, we suggest that either social-cognitive models incorporate implicit attitudes as a predictor for intention and behavior or dual-process models incorporate intention next to behavior or both.

Concerning the interactive pattern, which was present for intention prediction regarding both behaviors, it was again demonstrated that the effect of the explicit cognition on intention was strengthened when the implicit attitude and the respective explicit cognition (e.g. selfefficacy or perceived cons) were in the same direction. However, for the explicit constructs social modeling and social norms, a different pattern was detected. More specifically, perceiving many important others as sufficiently physically active (social modeling) had a stronger effect on the intention to be active, when the implicit attitude towards PA was negative. Regarding $\mathrm{RCM}$, the perceived expectation of important others to reduce one's RMC (social norms) had a stronger effect on intention to reduce one's consumption, when the implicit attitude towards red meat was negative or positive, but not when it was neutral. This suggests that dissonance between explicit constructs (in this case social norms and social modeling) and a person's implicit attitude (a) can exist and (b) may motivate individuals to resolve it. To do so, individuals probably neglected their implicit attitude and acted in accordance with the more accessible perceived norms and behavior (modeling) of important others, which resulted in a positive outcome from a health perspective in both studies, i.e. a higher intention to be active or a higher intention to reduce one's RMC. Thus, the dissonance between implicit attitudes and the respective explicit cognitions (social norms, social modeling) seemingly motivated people to act in accordance with the more obvious social influence, in this context resulting in desirable outcomes. This finding should, however, be regarded cautiously as social influence can also be negative, i.e. low modeling of a positive behavior or low expectations of important others to perform a healthy behavior. In this context, it is unclear whether dissonance (i.e. 
positive implicit attitude towards the behavior but low social influence) would have resulted in the same desirable outcome as a result of dissonance reduction.

Also, the discrepancy between implicit and explicit attitudes (IED) has been shown to be disadvantageous (Chapter 5). More precisely, IED was negatively associated with activity levels. Hence, the more dissonant implicit and explicit attitudes are, the less physically active the individual was. This could be due to an uncertainty about whether or not to engage in a behavior, which in turn stems from inconsistent attitudes towards the same behavior (e.g. liking PA explicitly but having negative feelings towards it implicitly or vice versa). These findings suggest that (a) IED can be detrimental for pursuing health behaviors and (b) that the congruence of attitudes should be taken into account when aiming at behavior change. Although implicit attitudes and explicit attitudes were not directly associated with behavior, the dissonance between both attitudes was. This was also found in another study (Goldstein et al., 2014). Contrary to our hypothesis, we did not find IED to moderate the influence of explicit attitude on PA intention or behavior (Chapter 5). Hence, IED did not weaken the predictive power of explicit attitudes on intention and behavior, but showed a direct negative effect on both these constructs.

As IED can have a negative effect on the pursuit of health behaviors, we consequently looked into possible factors that might (a) be able to reduce IED and (b) might be able to buffer a possible negative effect of IED on behavior and intention. Mindfulness was identified as a possible factor, but appeared not to be associated with IED (Chapter 4). What we did find, however, was that the subskill acceptance moderated the effect of IED on intention. That is, higher IED was associated with a higher intention to reduce one's RMC, which was probably the result of an attempt to reduce dissonance. This was, however, no longer the case when the subskill acceptance was strongly distinct. Hence, acceptance seemingly offers a skill to deal with the experienced dissonance and possible accompanying negative feelings. This was unidentified in the literature before and offers new avenues on how dissonance could be dealt with.

In Chapter 6, we looked at the pathways of implicit attitudes, explicit attitudes, and behavior (PA and RMC), and if and how these constructs relate to each other over time. Except for one relationship between implicit attitude and perceived cons (regarding PA), all pathways between these constructs were non-significant. This might indicate, that implicit attitudes and explicit attitudes are rather unrelated constructs, which do not shape each other but are shaped by other constructs. This is in line with the postulation of other authors, who claimed that implicit attitudes and explicit attitudes are rather independent constructs (e.g. Blair, 2001; Hofmann, Gschwendner, Nosek, et al., 2005; Nosek, 2005). Other authors, however, had opposing conclusions, e.g. that implicit attitudes and explicit attitudes are indeed related to each other (e.g. Gawronski \& Bodenhausen, 2006; Gschwendner et al., 2006; Hofmann, Gschwendner, Nosek, et al., 2005) and that, for example, implicit attitudes are used as a basis to form one's explicit attitude. Yet, this was not found in our study. 


\section{WHERE SHOULD IMPLICIT ATtitudes Be ADded IN A MODEL?}

The development of implicit measures has led to the discussion whether implicit attitudes and explicit attitudes are different types of attitudes and whether a conceptual distinction between the two types of attitudes is necessary or not (e.g. Fazio \& Olson, 2003). When looking at the definitions of both types of attitudes, the theoretical difference is quite clear: explicit attitudes are defined as overall evaluations that are based on cognitive, affective, and behavioral information, which people can report and whose activation can be controlled consciously. Implicit attitudes, on the other side, are defined as "introspectively unidentified (or inaccurately identified) traces of past experience that mediate favorable or unfavorable feeling, thought, or action toward a social object" (Greenwald \& Banaji, 1995, p. 8). Hence, whereas explicit attitudes tap into a person's reflections, implicit attitudes tap into automatically arising associations. According to theses definitions, it seems that an individual can have two types of attitudes regarding the same object. The fact that there can be discrepancy between the two types of attitudes might indicate that they are different constructs (i.e. otherwise they would be consistent). Also low correlations between the two types of attitudes in our studies as well as in former studies (e.g. Blair, 2001; Hofmann, Gschwendner, Nosek, et al., 2005; Nosek, 2005), might be an indication for this assumption.

A clear conclusion about whether implicit attitudes and explicit attitudes are indeed independent constructs or the same construct, which is 'simply' measured differently, is hard to draw at the moment. However, our studies have shown that they 'behave' differently in the prediction of behavior than explicit attitudes do. That is, implicit attitudes moderated the relationships between certain explicit cognitions (e.g. perceived cons, self-efficacy, social norms) and intention as well as between explicit cognitions (e.g. self-efficacy) and behavior. These patterns are unknown for explicit attitudes. Hence we postulate that implicit attitudes, when aiming to integrate them in a social-cognitive model, cannot and should not be added at the same place where explicit attitudes are normally located (e.g. as antecedent for intention). Although, we found implicit attitudes to be associated with intention (to reduce RMC) - a pattern that applies to explicit attitudes - even more patterns of influence were found (as outlined above). Furthermore, other studies found implicit attitudes to also have a direct influence on behavior and to predict behavior above and beyond explicit attitudes (e.g. Conroy et al., 2010; Friese et al., 2007; Maison et al., 2004). Due to these differences, we argue that implicit attitudes, when added in a social-cognitive model e.g. the I-change model (De Vries, 2017), should not be added at the same spot where explicit attitudes are located. A possible outline of where implicit attitudes could be added in (one part of) the I-change model, is depicted in figure 7.1. However, we stress the importance of more follow-up studies in order to be able to draw conclusions regarding causal relationships, e.g. between explicit attitudes and implicit attitudes and whether one type of attitude proceeds the other or not. Moreover, since the I-change model is a model, which depicts the enactment of behavior in multiple phases (e.g. 
pre-motivational, motivational, post-motivational), clarification is needed regarding the exact phase where implicit attitudes need to be nested. Although figure 7.1 might give an indication on where implicit attitudes could be added in a model, this suggestion should not be regarded as an 'end product' but rather as a working model where future studies could build on.

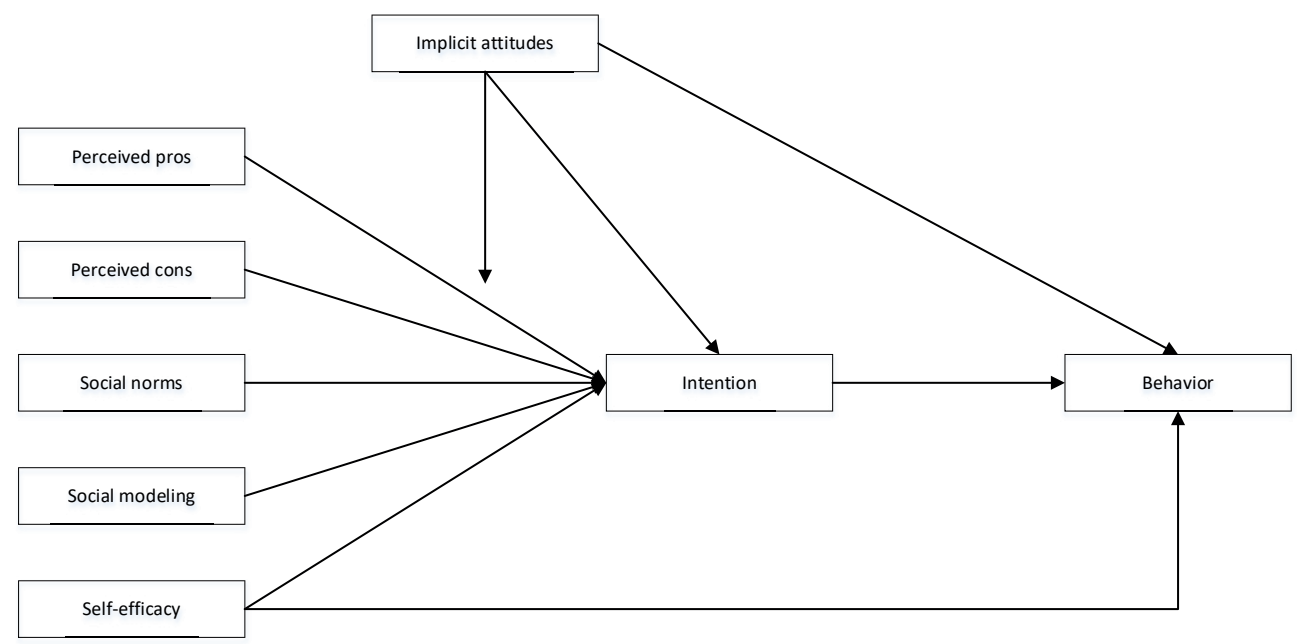

Figure 7.1 | Implicit attitudes as moderator of the relationships between explicit cognitions and intention/behavior and as direct predictor for intention and behavior

Our results have shown that implicit attitudes and explicit attitudes can have an even stronger effect on intention and behavior, when they are consistent. How consistency between the two constructs can be achieved, will therefore be discussed in the following.

\section{HOW TO ACHIEVE CONSISTENCY BETWEEN IMPLICIT ATTITUDES AND EXPLICIT COGNITIONS?}

According to our results (Chapter 2, Chapter 3, and Chapter 5) and to Nosek et al. (2011), implicit attitudes and explicit cognitions have a stronger impact on behavior and intention, when they point into the same direction - i.e. when they mutually reinforce each other. Therefore, it appears reasonable that interventions, which are aiming at behavior change, should (a) target both implicit attitudes as well as explicit cognitions and (b) should aim to achieve consistent explicit cognitions and implicit attitudes. In order to achieve consistency between the two types of cognitions, different approaches could be used and are presented in the following. 


\section{Approach 1:}

\section{Changing implicit attitudes and explicit cognitions separately from each other}

As mentioned earlier, implicit attitudes and explicit cognitions are assumed to originate from different underlying processes - implicit attitudes are influenced by automatic and rather unconscious mechanisms whereas explicit cognitions are steered by deliberate, reasoned, and rather conscious processes (Olson \& Fazio, 2006; Rydell \& McConnell, 2006; Strack \& Deutsch, 2004). Therefore, addressing implicit attitudes and explicit cognitions require different approaches. There exists a variety of scientifically tested methods to change explicit cognitions, such as arguments to change explicit attitudes, role modeling to change self-efficacy, or gathering information about others' approval to change social norms (e.g. Bartholomew Eldredge et al., 2016). Although Bartholomew Eldredge et al. (2016) also suggested methods to modify automatic behaviors, e.g. by means of implementation intentions or cue altering, it is debatable whether implicit and automaticity concern the same processes or not (Nosek \& Greenwald, 2009; Nosek et al., 2011) and whether they are changeable by means of the same methods or not, i.e. could implicit attitudes be changed by means of implementation intentions or cue altering? Currently, there is no scientific support that implicit cognitions can be changed by means of the aforementioned methods concerning automatic behaviors. Moreover, implicit attitudes were believed to be hard to change for a long time (Wilson et al., 2000) as they are attributed to the slow-learning system. In this system, learning takes place by means of a slow accumulation of associative information over time (Sloman, 1996). Until now, a few studies have addressed the alteration of implicit attitudes and the paradigm that is normally used within these studies is evaluative conditioning (De Houwer, Thomas, \& Baeyens, 2001). Evaluative conditioning causes a positive or negative change in the valence of an object that results from pairing the object with another either positive or negative stimulus (Hofmann, De Houwer, Perugini, Baeyens, \& Crombez, 2010). Hence, the attitude change is a result of the co-occurrence of the object with another valence laden object (Jones, Olson, \& Fazio, 2010). For example, Hollands and colleagues (2011) presented images of snacks paired with images of potential negative health consequences, which resulted in more negative implicit attitudes towards snacks. This was especially the case when the previous implicit attitudes towards snacks were favorable. Mattavelli et al. (2017) demonstrated that implicit attitudes can also be modified into a positive direction. That is, people who performed an evaluative conditioning task, in which green vegetables were repeatedly paired with positive stimuli, showed more positive implicit attitudes towards green vegetables than a control group. These changes were present after having performed the task only once. In a different study, the performance of one IAT, in which participants were instructed to pair the word 'I' with positive words, led to higher implicit self-esteem (Ebert et al., 2009). Hence, contrary to the longbelieved assumption that implicit attitudes are difficult to change, implicit attitude change can happen relatively quickly without requiring a lot of time. Moreover, it has been shown that the learned associations between the object and the valenced stimulus persist even when the object is encountered in the absence of the valenced stimulus. For example, referring to the 
aforementioned object green vegetables, it is likely that they will still be evaluated as positive when they are encountered in a setting where the stimuli used in the study (e.g. words or pictures) are not present, e.g. in a supermarket or in a restaurant. Especially advertisements make use of this mechanism (Walther, Ebert, \& Meinerling, 2011).

In sum, implicit attitudes can be changed into a positive or negative direction. A suitable method to do so is evaluative conditioning. This method should be complemented by suitable evidence-based methods to change explicit cognitions. Thereby a change in both types of cognitions is performed separately from each other. To our knowledge, only the study of Mattavelli (2017) has used this approach until now, however only partly. Although they changed implicit attitudes towards green vegetables by means of an evaluative learning task and explicit attitudes by means of persuasive communication, other explicit cognitions, such as self-efficacy or social norms, were not addressed. Although the intervention resulted in modified implicit and explicit attitudes and a heightened readiness to change one's consumption of green vegetables, we suggest that it is also important to address the remaining explicit cognitions, which are also known to be related to intention and behavior as this might make the occurrence of the desired health behavior even more likely.

\section{Approach 2:}

\section{Changing implicit attitudes and fostering the skill to translate them into explicit attitudes}

According to the current scientific knowledge, a second option to achieve consistency would be to change implicit attitudes only. This is based on the following reasoning. It is believed that individuals are unaware of the underlying processes of implicit attitude formation; however, they might be aware of the automatic reaction towards an object or behavior (e.g. positive or negative feelings or a 'gut feeling') (Gawronski \& Bodenhausen, 2006). When individuals classify these automatic reactions as valid and take them into account when forming their explicit cognitions, implicit attitudes and explicit cognitions might change correspondingly as depicted in the Associative-Propositional Evaluation Model (APE; Gawronski \& Bodenhausen, 2006). Indeed, Grumm and colleagues (2009) revealed that changes in the implicit attitude can lead to changes in the explicit measures as postulated by the APE. That is, participants who were first exposed to an evaluative conditioning task and afterward instructed to think about their feelings when answering an explicit measure, showed changes in the implicit as well as in the explicit measure. This was not the case in a control group, in which participants were asked to focus on their knowledge when answering the explicit measure. Although under specific circumstances, a change in one type of cognition (i.e. implicit) can indeed result in a change in the other type of cognition (i.e. explicit), this process cannot and should not be taken for granted. That is, other studies showed that manipulations concerning the implicit attitude only resulted in the change of the implicit attitude but not in a change of the explicit attitude (e.g. Grumm et al., 2009; Rydell \& McConnell, 2006).

According to the APE (Gawronski \& Bodenhausen, 2006), it should also be possible that a change in explicit attitude could lead to a corresponding change in a person's implicit 
attitude. More specifically, it is assumed that newly acquired information regarding a specific object will be reflected in the explicit attitude towards the object. This process might lead to the formation of new associations between the object (e.g. smoking) and its evaluation (e.g. negative), which should be represented in the person's implicit attitude towards the object (e.g. a negative implicit attitude towards smoking). A few studies have shown that methods to change explicit attitudes resulted only in a change of the respective explicit attitude but not the implicit attitude (e.g. Baccus, Baldwin, \& Packer, 2004; Gawronski \& Strack, 2004). Hence, this approach should be regarded as critical until it is scientifically confirmed.

Hence, interventions could change implicit attitudes only and at the same time foster the ability to translate one's implicit attitude (i.e. feelings) into one's explicit realities. Which specific ability should be fostered is, however, unclear at the moment, since findings regarding introspective access and mindfulness - two skills that were discussed to enable a better translation of implicit attitudes into explicit statements - showed mixed findings. Whereas these abilities led indeed to more congruent implicit and explicit attitudes in some studies (Brown \& Ryan, 2003; Koole et al., 2009), other studies could not find support (Hyde et al., 2010; Chapter 4). Exact causes for these mixed results are unclear at this point in time. Therefore, more studies are needed in order to pinpoint which skill(s) lead to a better translation of one's implicit evaluations into one's explicit evaluations. Moreover, this approach concerns 'only' the translation of the implicit attitude into an explicit attitude. As mentioned before, behavior and intention are well-known to be associated with more explicit cognitions besides explicit attitude. Thus, when using this approach, additional measures would be necessary to change the remaining explicit cognitions, such as social norms or self-efficacy.

\section{Approach 3:}

\section{Dissonance induction}

An alternative approach might be through dissonance induction. Dissonance between implicit attitudes and explicit cognitions can stimulate people to act in a certain way as individuals are motivated to reduce dissonance (Festinger, 1957). The findings regarding dissonance and their resulting consequences are, however, mixed, e.g. in some studies, dissonance led to the desired outcomes (Berry et al., 2011; Chapter 4; Gawronski \& Strack, 2004; Rydell et al., 2008), whereas this was the opposite in other studies (Goldstein et al., 2014; Maliszewski, 2011; Chapter 5). Also, it is unclear whether dissonance leads to a change in cognitions or 'only' in a change of behavior. Although behavior change is the ultimate goal of health promoters, it is unclear whether the behavior is maintained over time as a result of dissonance reduction. To our knowledge, this question has not yet been addressed in studies thus far. Hence, before defining and applying dissonance induction as a method to achieve behavior change, more studies are necessary in order to identify whether dissonance does indeed lead to a change in cognitions and furthermore, under which specific circumstance this approach can be beneficial or detrimental for behavior change interventions. As research regarding this filed is still in its infancies, more studies are needed in order to draw firm conclusions on how and under which 
circumstances dissonance induction would work in order to change behavior in the long term. Aspects that could be investigated are, for instance, the direction of the pre-existing attitudes (e.g. implicit positive and explicit negative and vice versa) and whether and how this affects dissonance reduction and ultimately behavior.

Summarizing, based on the findings of this dissertation and the findings of the aforementioned studies, there seemingly exist three different strategies in order to achieve consistency between implicit attitudes and explicit cognitions. It is important to state that independent of which of the aforementioned approaches is followed, interventions should always look at the individual's pre-existing cognitions first. Depending on the initial cognitions, only those cognitions that are not in a favorable direction towards the behavior should be modified - e.g. both types of cognitions should be modified (when implicit attitudes and other relevant explicit cognitions are unfavorable regarding the behavior) or only one type of cognition should be modified (implicit attitudes are unfavorable but other relevant explicit cognitions are favorable towards the behavior or vice versa). Rather than basing interventions on the assumption that certain cognitions need to be changed, thereby putting oneself at risk for developing programs that do not succeed, this tailored approach would increase the potential effectiveness of health behavior change programs (Bartholomew Eldredge et al., 2016).

We conclude that, when implicit attitudes are not in the desired direction, they can and should be altered. As implicit attitudes are often classified as 'unconscious' attitudes, we will discuss in the next paragraph whether implicit attitudes are rightly considered as 'unconscious' as well as the implications of the resulting conclusion.

\section{ARE IMPLICIT ATTITUDES UNCONSCIOUS?}

In the current scientific literature, there is an ongoing discussion about whether implicit attitudes are unconscious or not (e.g. De Houwer, 2006; Gawronski, 2009; Gawronski, Hofmann, \& Wilbur, 2006; Hahn \& Gawronski, 2014; Hahn, Judd, Hirsh, \& Blair, 2014; Krickel, 2018). This is a relevant question, because when a person is unaware about the fact that a given factor - his or her implicit attitude - influences his or her judgments, a correction of this factor is impossible, even when the person is motivated to control for a possible influence of this factor. Major drivers that led to the assumption that implicit attitudes are unconscious, were the findings that implicit attitudes and explicit attitudes are often weakly correlated (e.g. Blair, 2001; Hofmann, Gschwendner, Nosek, et al., 2005; Nosek, 2005) and that they explain unique aspects of behavior across various domains that are not revealed by traditional self-reports (e.g. Friese et al., 2008; Greenwald et al., 2009; Rydell \& McConnell, 2006). Furthermore, the discovery that implicit and explicit attitudes can be dissonant from each other has led some researchers to argue that implicit attitudes cannot be introspected on (e.g. Devos, 2008; McConnell, Dunn, Austin, \& Rawn, 2011; Spalding \& Hardin, 1999). 
Other authors argued that people do indeed have access to their implicit attitudes as correlations between implicit and explicit attitudes hardly ever equal zero, suggesting that some implicit information must be available to an individual when answering explicit measures (e.g. Gschwendner et al., 2006; Hofmann, Gschwendner, Nosek, et al., 2005). As outlined in the introduction of this dissertation (Chapter 1), other studies demonstrated that the size of the correlation between implicit attitudes and explicit attitudes can vary as a function of different factors (for a review, see: Gawronski et al., 2006), such as attitude importance (Hofmann, Gschwendner, Nosek, et al., 2005), a person's preference for consistency (Thrash et al., 2007), or methodological factors (Hofmann, Gschwendner, Nosek, et al., 2005). The claim that implicit attitudes are unconscious has mainly been based on theoretical assumptions, rather than on scientific evidence. A more novel study, however, showed that participants were able to predict their performance on an implicit task as well as their implicit evaluations when asked to do so, even when they reported different explicit attitudes towards the same target (Hahn et al., 2014). Hence, although there exists no common agreement in the literature about the unconsciousness of implicit attitudes, the latest scientific results indicate that people can indeed be aware of the content of their implicit attitude (i.e. positive, negative, neutral), when they are asked to predict them. Therefore, implicit attitudes should rather be defined as accessible to consciousness to some degree than as unconscious attitudes.

As individuals might be aware of the content of their implicit attitudes, the more important question that we argue should be asked is: Are people aware of the impact that implicit attitudes have on their behavior? We argue that the awareness of certain factors, including the content of one's implicit attitude, does not necessarily entail that a person does or does not react accordingly - comparable to a smoker who is aware of the negative consequences of smoking but does not react in accordance with his knowledge and continues smoking. Indeed, a study by Gawronski et al. (2006) indicated that participants are well able to predict the impact that their explicit attitudes have on their behavior, but not the impact that their implicit attitudes have on the same behavior (impact awareness). Hence, although participants seem to be able to predict the content of their implicit evaluations (Hahn \& Gawronski, 2014), the impact that their evaluations have on their behavior remains rather unconscious (Gawronski et al., 2006). This conclusion has important implications for further research as a lack of impact awareness of implicit attitudes on undesired behaviors needs to be tackled differently (e.g. by means of awareness creation) than for example a lack of content awareness or the ability or motivation to control the effects of one's implicit attitudes on behavior (e.g. by increasing self-control or executive functioning).

\section{LIM ITATIONS}

When interpreting the findings of this dissertation, the following limitations should be taken into account. Firstly, for the assessment of behavior, self-report measures were used. Although 
self-reports are widely accepted tools to measure behavior and validated tools were used, self-reports are also prone to reporting errors and are therefore defined as less reliable. We did not find direct relations between implicit attitudes and behavior, which was however the case in other studies (e.g. Arcuri et al., 2008; Conroy et al., 2010; McConnell \& Leibold, 2001; Prestwich et al., 2011). In these studies, behavior was assessed by means of more objective measures, such as pedometers, actual food choice/consumption, or social interactions. It could be that the absence of a direct relationship between implicit attitude and behavior in our studies is a result of the self-report measures, which did not depict the 'true' relationship between these two elements.

Secondly, in the study on RMC, we assessed implicit attitudes towards the object (red meat) and not towards the behavior (consumption of red meat). Explicit cognitions, however, were assessed towards the behavior (consumption of red meat). This was mainly due to practical reasons as it appeared rather difficult to identify stimuli that clearly represented the consumption of red meat (e.g. pictures or words). Therefore, and based on other studies, which had to deal with the same issue (Huijding et al., 2005; Waters et al., 2007), we assessed the implicit attitude towards the object (red meat). However, attitudes are known to show a stronger relationship with behavior when both behavior and attitudes are measured regarding the same target (e.g. implicit attitudes towards RMC and explicit attitudes towards RMC) (Ajzen \& Fishbein, 1980; Wicker, 1969). The chosen approach might have led to a lower correspondence between the implicit attitude and the explicit cognitions, with inaccurate depictions of the relationships between these cognitions and behavior as well as an incorrect depiction of IED as a possible result. In order to conclude whether this was the case, we encourage future studies to compare whether the selection of stimuli, which more clearly depict the behavior (e.g. pictures in which a piece of red meat is clearly directed towards the mouth), leads to different results regarding the abovementioned relationship, than when stimuli representing the target object (e.g. red meat) are used.

Thirdly, in our studies, we focused on a particular measure of implicit attitudes, namely the Single-Category Implicit Association Task (SC-IAT; Karpinski \& Steinman, 2006) because it was the most suitable tool. That is, contrary to the Implicit-Association Task (IAT; Greenwald et al., 1998), the most widely used implicit measure, it allowed us to assess implicit attitudes towards an object unrelated to another object. However, there exist more measures that also asses implicit attitudes, such as the affective priming task (Fazio, Sanbonmatsu, Powell, \& Kardes, 1986), the Extrinsic Affective Simon Task (De Houwer, 2003) or the Go/No-Go Association Task (Nosek \& Banaji, 2001). As the SC-IAT has demonstrated the highest reliability (usually between .70-.90; Karpinski \& Steinman, 2006) and internal consistency compared to the other latency-based implicit measures, it was selected for the studies of this dissertation. The different measures are, however, not highly correlated with each other (Bar-Anan \& Nosek, 2012; Olson \& Fazio, 2003), indicating that different tests capture different facets of implicit attitudes. Hence, it is unclear if and how the use of different measures would have affected our findings. 
Fourthly, implicit attitudes were selected as a representative of implicit cognitions. However, other implicit cognitions such as approach or avoidance tendencies or attentional biases also exist in the family of implicit cognitions. It is unclear whether these implicit cognitions show the same or different relationships that were investigated in the studies of this dissertation. As these patterns were not examined, we advise against a generalization of the findings of this dissertation to other implicit cognitions.

Fifthly, we used the core of the I-change model (De Vries, 2017), which is broadly in line with the widely used Reasoned Action Approach (Fishbein \& Ajzen, 2011), as a framework to look into different patterns between implicit attitudes and explicit cognitions regarding intention and behavior prediction. By focusing on the core only, other (more distal) determinants that are depicted in the model and are inspired from other health models, e.g. risk perception, or predisposing factors such as psychological factors (e.g. personality traits such as impulsivity), were excluded. It is possible that these determinants also interplay with implicit attitudes. For example, it has been shown that implicit associations are more strongly related to behavior for people, who score higher on certain facets of impulsivity (Burton, Pedersen, \& McCarthy, 2012). This knowledge stems mainly from addiction research. Shedding knowledge on whether there is also an interaction between trait impulsivity and implicit associations in the area of health promotion seems worthwhile.

\section{SUGGESTIONSFOR FUTURE DIRECTIONS}

Our results indicate that similar patterns of how implicit attitudes and explicit cognitions jointly predict intention and behavior apply across two different types of behavior. In order to draw, however, more generalizable conclusions, more studies with different behaviors preferably objectively measured behavior - should follow. Only when the same patterns are found across various (health) behaviors, implicit attitudes can be added correspondingly in a (health) behavior model.

Moreover, as we found implicit attitudes and explicit cognitions to be directly and indirectly related to intention and behavior, studies should investigate whether targeting both implicit attitudes and explicit cognitions results indeed in stronger behavioral changes compared to when only one type of cognition is tackled within an intervention. Associated with this research question comes the question of what the best method would be to achieve a change in both types of cognitions. As discussed above, there exist different ways, such as applying different methods to change implicit attitudes and explicit cognitions separately or changing implicit attitudes and at the same time fostering the skill that leads to a better translation of implicit associations into explicit cognitions. In order to decide on the most effective method, studies should compare these approaches.

In the context of implicit measures research, we encourage studies to investigate whether the association between implicit attitudes and behavior increases when both the implicit 
measure and the behavior are assessed on the same level - i.e. implicit attitudes towards the behavior and not towards the object that the behavior entails. Additionally, more insight into possible effects of the stimuli that are used in the implicit measures would be worthwhile. The common approach is to use sterile and neutral pictures to represent a target (e.g. a glass of beer on a white background). For many people, however, certain behaviors, such as drinking alcohol is a social happening and depending on the social environment people either tend to drink more or less alcohol (Cutler \& Storm, 1975). Although there exist a few studies regarding the wording of stimuli and categories in implicit measures, especially for the IAT, (Bluemke \& Friese, 2006; Govan \& Williams, 2004; Mitchell, Nosek, \& Banaji, 2003), studies on how to best depict stimuli and a possible influence on performance are scarce. It is conceivable that a neutral glass of beer depicted on a white background could trigger different implicit associations compared to a glass of beer presented in a group of people. Hence, future studies should compare whether the results of an implicit measure changes depending on how stimuli are depicted, e.g. neutral stimuli versus stimuli depicted in a more natural setting. More studies are needed in order to create a generic rule for the selection of stimuli, which does not exist at present.

\section{CONCLUSION}

Implicit attitudes interact with well-known explicit determinants (perceived cons, social influence, self-efficacy) in the prediction of behavior and intention across two different health behaviors, thereby offering support for the interactive pattern. For intention also support for the additive pattern was found for one health behavior indicating that the two patterns do not exclude each other. Further, IED was found to be detrimental for the pursue of one health behavior, which might probably be due to an indecisiveness of whether to move towards the behavior or not, resulting from dissonant implicit and explicit attitudes. Hence, besides modifying explicit cognitions, interventions might profit from also altering implicit attitudes into the same direction, thereby reinforcing the beneficial effects that the explicit cognitions have on intention and behavior. The best approach to align implicit attitudes and explicit cognitions with each other is unexplored at present but should be the focus of future studies. Mindfulness was unrelated to the degree of dissonance between implicit and explicit attitudes. However, the subskill acceptance seemingly offers a way to deal with this dissonance, which was unidentified in the literature before and offers a new and alternative approach to handle dissonance. Moreover, implicit attitudes and explicit attitudes were shown to be unrelated over time for two different types of behaviors. Hence, both types of attitudes seem to be rather independent constructs, which do not influence each other but are shaped by other constructs. 


\section{References}

Adams, S. A., Matthews, C. E., Ebbeling, C. B., Moore, C. G., Cunningham, J. E., Fulton, J., \& Hebert, J. R. (2005). The Effect of Social Desirability and Social Approval on Self-Reports of Physical Activity. American Journal of Epidemiology, 161(4), 389-398. doi:10.1093/aje/kwi054

Aiken, L. S., West, S. G., \& Reno, R. R. (1991). Multiple regression: Testing and interpreting interactions: Sage.

Aiking, H. (2014). Protein production: planet, profit, plus people? The American Journal of Clinical Nutrition, 100(suppl_1), 483S-489S. doi:10.3945/ajcn.113.071209

Ainsworth, B. E., Haskell, W. L., Leon, A. S., Jacobs, D. R., Jr., Montoye, H. J., Sallis, J. F., \& Paffenbarger, R. S., Jr. (1993). Compendium of physical activities: classification of energy costs of human physical activities. Medicine and Science in Sports and Exercise, 25(1), 71-80.

Ajzen, I. (1991). The theory of planned behavior. Organizational Behavior and Human Decision Processes, 50(2), 179-211. doi:https://doi.org/10.1016/0749-5978(91)90020-T

Ajzen, I. (2002). Constructing a TPB questionnaire: Conceptual and methodological considerations.

Ajzen, I., \& Fishbein, M. (1977). Attitude-behavior relations: A theoretical analysis and review of empirical research. Psychological Bulletin, 84(5), 888.

Ajzen, I., \& Fishbein, M. (1980). Understanding attitudes and predicting social behavior. New Jersey: Prentice-Hall Inc.

Albarracin, D., Johnson, B. T., Fishbein, M., \& Muellerleile, P. A. (2001). Theories of reasoned action and planned behavior as models of condom use: a meta-analysis. Psychological Bulletin, 127(1), 142.

Albarracín, D., \& Wyer Jr, R. S. (2000). The cognitive impact of past behavior: Influences on beliefs, attitudes, and future behavioral decisions. Journal of Personality and Social Psychology, 79(1), 5-22. doi:10.1037/0022-3514.79.1.5

Albert, B. (1986). Social foundations of thought and action: A social cognitive theory. NY.: Prentice-Hall.

Arcuri, L., Castelli, L., Galdi, S., Zogmaister, C., \& Amadori, A. (2008). Predicting the vote: Implicit attitudes as predictors of the future behavior of decided and undecided voters. Political Psychology, 29(3), 369-387.

Armitage, C. J., \& Conner, M. (2001). Efficacy of the theory of planned behaviour: A meta-analytic review. British Journal of Social Psychology, 40(4), 471-499.

Aronson, E. (1992). The return of the repressed: Dissonance theory makes a comeback. Psychological inquiry, 3(4), 303-311. doi:10.1207/s15327965pli0304_1

Baccus, J. R., Baldwin, M. W., \& Packer, D. J. (2004). Increasing implicit self-esteem through classical conditioning. Psychological Science, 15(7), 498-502.

Baer, R. A., Smith, G. T., \& Allen, K. B. (2004). Assessment of Mindfulness by Self-Report:The Kentucky Inventory of Mindfulness Skills. Assessment, 11(3), 191-206. doi:10.1177/1073191104268029

Baer, R. A., Smith, G. T., Hopkins, J., Krietemeyer, J., \& Toney, L. (2006). Using Self-Report Assessment Methods to Explore Facets of Mindfulness. Assessment, 13(1), 27-45. doi:10.1177/1073191105283504

Bagozzi, R. P., \& Warshaw, P. R. (1990). Trying to consume. Journal of consumer research, 17(2), 127-140.

Bar-Anan, Y., \& Nosek, B. A. (2012). A comparative investigation of seven implicit measures of social cognition.

Bar-Haim, Y., Lamy, D., Pergamin, L., Bakermans-Kranenburg, M. J., \& van ljzendoorn, M. H. (2007). Threatrelated attentional bias in anxious and nonanxious individuals: A meta-analytic study. Psychological Bulletin, 133(1), 1-24. doi:10.1037/0033-2909.133.1.1

Bargh, J. A., \& Chartrand, T. L. (2000). The mind in the middle. Handbook of research methods in social and personality psychology, 253-285.

Barnes-Holmes, D., Murtagh, L., Barnes-Holmes, Y., \& Stewart, I. (2010). Using the Implicit Association Test and the Implicit Relational Assessment Procedure to Measure Attitudes Toward Meat and Vegetables in Vegetarians and Meat-Eaters. The Psychological Record, 60(2), 287-305. doi:10.1007/ bf03395708 
Bartholomew Eldredge, L. K., Markham, C. M., Ruiter, R. A., Fernández, M. E., Kok, G., \& Parcel, G. S. (2016). Planning health promotion programs: an intervention mapping approach: John Wiley \& Sons.

Bédard, N. J. M., Paul Krueger, Kevin Brazil, Michel. (2000). Assessing reproducibility of data obtained with instruments based on continuous measurements. Experimental Aging Research, 26(4), 353-365.

Begh, R., Munafò, M. R., Shiffman, S., Ferguson, S. G., Nichols, L., Mohammed, M. A., . . Aveyard, P. (2013). Attentional bias retraining in cigarette smokers attempting smoking cessation (ARTS): Study protocol for a double blind randomised controlled trial. BMC Public Health, 13(1), 1176.

Benjamini, Y., \& Hochberg, Y. (1995). Controlling the false discovery rate: a practical and powerful approach to multiple testing. Journal of the Royal statistical society: series B (Methodological), 57(1), 289-300.

Benjamini, Y., \& Yekutieli, D. (2001). The control of the false discovery rate in multiple testing under dependency. The annals of statistics, 29(4), 1165-1188.

Berndsen, M., \& van der Pligt, J. (2005). Risks of meat: the relative impact of cognitive, affective and moral concerns. Appetite, 44(2), 195-205. doi:https://doi.org/10.1016/j.appet.2004.10.003

Berry, T. R. (2016). Changes in implicit and explicit exercise-related attitudes after reading targeted exercise-related information. Psychology of Sport and Exercise, 22, 273-278. doi:10.1016/j. psychsport.2015.09.001

Berry, T. R., Jones, K. E., McLeod, N. C., \& Spence, J. C. (2011). The relationship between implicit and explicit believability of exercise-related messages and intentions. Health Psychology, 30(6), 746.

Berry, T. R., Rodgers, W. M., Divine, A., \& Hall, C. (2018). The Relationship of Explicit-Implicit Evaluative Discrepancy to Exercise Dropout in Middle-Aged Adults. Journal of Sport and Exercise Psychology, $20(\mathrm{XX}), 1-9$.

Berry, T. R., Rodgers, W. M., Markland, D., \& Hall, C. R. (2016). Moderators of implicit-explicit exercise cognition concordance. Journal of Sport and Exercise Psychology, 38(6), 579-589.

Biddle, S. J., \& Mutrie, N. (2007). Psychology of physical activity: Determinants, well-being and interventions: Routledge.

Bingham, C. R., Barretto, A. I., Walton, M. A., Bryant, C. M., Shope, J. T., \& Raghunathan, T. E. (2011). Efficacy of a Web-Based, Tailored, Alcohol Prevention/Intervention Program for College Students: 3-Month Follow-up. Journal of Drug Education, 41(4), 405-430. doi:10.2190/DE.41.4.e

Bizer, G. Y., \& Krosnick, J. A. (2001). Exploring the structure of strength-related attitude features: The relation between attitude importance and attitude accessibility. Journal of Personality and Social Psychology, 81(4), 566.

Blair, I. V. (2001). Implicit stereotypes and prejudice. Paper presented at the Cognitive social psychology: The Princeton symposium on the legacy and future of social cognition.

Bluemke, M., Brand, R., Schweizer, G., \& Kahlert, D. (2010). Exercise might be good for me, but I don't feel good about it: do automatic associations predict exercise behavior? Journal of Sport and Exercise Psychology, 32(2), 137-153. doi:10.1123/jsep.32.2.137

Bluemke, M., \& Friese, M. (2006). Do features of stimuli influence IAT effects? Journal of Experimental Social Psychology, 42(2), 163-176.

Bluemke, M., \& Friese, M. (2008). Reliability and validity of the Single-Target IAT (ST-IAT): assessing automatic affect towards multiple attitude objects. European Journal of Social Psychology, 38(6), 977-997.

Boendermaker, W. J., Peeters, M., Prins, P. J., \& Wiers, R. W. (2017). Using serious games to (re) train cognition in adolescents Serious games and edutainment applications (pp. 307-321): Springer.

Boninger, D. S., Krosnick, J. A., \& Berent, M. K. (1995). Origins of attitude importance: self-interest, social identification, and value relevance. Journal of Personality and Social Psychology, 68(1), 61.

Bosson, J. K., Brown, R. P., Zeigler-Hill, V., \& Swann, W. B. (2003). Self-enhancement tendencies among people with high explicit self-esteem: The moderating role of implicit self-esteem. Self and Identity, 2(3), 169-187.

Bouvard, V., Loomis, D., Guyton, K. Z., Grosse, Y., Ghissassi, F. E., Benbrahim-Tallaa, L., . . Straif, K. (2015). Carcinogenicity of consumption of red and processed meat. The Lancet Oncology, 16(16), 15991600. doi:https://doi.org/10.1016/S1470-2045(15)00444-1 
Bradley, M. M., \& Lang, P. J. (1999). Affective norms for English words (ANEW): Instruction manual and affective ratings. Retrieved from http://citeseerx.ist.psu.edu/viewdoc/ download?doi=10.1.1.306.3881\&rep=rep1\&type $=$ pdf

Brand, R., \& Antoniewicz, F. (2016). Affective Evaluations of Exercising: The Role of Automatic-Reflective Evaluation Discrepancy. Journal of Sport and Exercise Psychology, 38(6), 631-638.

Brand, R., \& Schweizer, G. (2015). Going to the gym or to the movies?: situated decisions as a functional link connecting automatic and reflective evaluations of exercise with exercising behavior. Journal of Sport and Exercise Psychology, 37(1), 63-73.

Brinol, P., Petty, R. E., \& Wheeler, S. C. (2006). Discrepancies between explicit and implicit self-concepts: Consequences for information processing. Journal of Personality and Social Psychology, 91(1), 154170. doi:10.1073/0022-3514.91.1.154

Brochu, P. M., \& Morrison, M. A. (2007). Implicit and Explicit Prejudice Toward Overweight and AverageWeight Men and Women: Testing Their Correspondence and Relation to Behavioral Intentions. The Journal of Social Psychology, 147(6), 681-706. doi:10.3200/SOCP.147.6.681-706

Brown, K. W., \& Cordon, S. (2009). Toward a phenomenology of mindfulness: Subjective experience and emotional correlates Clinical handbook of mindfulness (pp. 59-81): Springer.

Brown, K. W., \& Ryan, R. M. (2003). The benefits of being present: mindfulness and its role in psychological well-being. Journal of Personality and Social Psychology, 84(4), 822.

Brown, K. W., \& Ryan, R. M. (2004). Perils and promise in defining and measuring mindfulness: Observations from experience. Clinical Psychology: Science and Practice, 11(3), 242-248.

Brown, K. W., Ryan, R. M., \& Creswell, J. D. (2007). Mindfulness: Theoretical foundations and evidence for its salutary effects. Psychological inquiry, 18(4), 211-237.

Brüll, P., Hebecker, R., Wiers, R. W., Kok, G., \& Ruiter, R. A. C. (under review). Heroes of

the Night - Promoting three safer-sex behaviors by targeting explicit and implicit

behavioral determinants in a serious game.

Burton, C. M., Pedersen, S. L., \& McCarthy, D. M. (2012). Impulsivity moderates the relationship between implicit associations about alcohol and alcohol use. Psychology of addictive behaviors : journal of the Society of Psychologists in Addictive Behaviors, 26(4), 766-772. doi:10.1037/a0028742

Calitri, R., Lowe, R., Eves, F. F., \& Bennett, P. (2009). Associations between visual attention, implicit and explicit attitude and behaviour for physical activity. Psychol Health, 24(9), 1105-1123. doi:10.1080/08870440802245306

Carfora, V., Caso, D., \& Conner, M. (2017a). Correlational study and randomised controlled trial for understanding and changing red meat consumption: The role of eating identities. Social Science \& Medicine, 175, 244-252.

Carfora, V., Caso, D., \& Conner, M. (2017b). Randomised controlled trial of a text messaging intervention for reducing processed meat consumption: The mediating roles of anticipated regret and intention. Appetite, 117, 152-160.

Chan, W., McCance, R. A., \& Brown, J. (1996). Meat products and dishes: Sixth supplement to the fifth edition of McCance and Widdowson's The Composition of Foods: Royal Society of Chemistry.

Chatzisarantis, N., Hagger, M., Biddle, S., \& Smith, B. (2005). The stability of the attitude-intention relationship in the context of physical activity. Journal of Sports Sciences, 23(1), 49-61. doi:10.1080/ 02640410410001730070

Chemnitz, C., \& Becheva, S. (2014). Meat atlas: Facts and figures about the animals we eat: Heinrich Böll Foundation.

Cheval, B., Sarrazin, P., Isoard-Gautheur, S., Radel, R., \& Friese, M. (2015). Reflective and impulsive processes explain (in)effectiveness of messages promoting physical activity: a randomized controlled trial. Health Psychol, 34(1), 10-19. doi:10.1037/hea0000102

Chevance, G., Héraud, N., Varray, A., \& Boiché, J. (2017). Change in explicit and implicit motivation toward physical activity and sedentary behavior in pulmonary rehabilitation and associations with postrehabilitation behaviors. Rehabilitation Psychology, 62(2), 119. 
Ciccomascolo, L. E., \& Grossi, L. M. (2008). The effect of an 8-week educational curriculum and physical activity program on attitudes toward physical activity and body image of urban adolescent girls. Women in Sport and Physical Activity Journal, 17(2), 17-23.

Clerkin, E. M., \& Teachman, B. A. (2010). Training implicit social anxiety associations: an experimental intervention. Journal of Anxiety Disorders, 24(3), 300-308. doi:10.1016/j.janxdis.2010.01.001

Cohen, J., Cohen, P., West, S. G., \& Aiken, L. S. (2013). Applied multiple regression/correlation analysis for the behavioral sciences: Routledge.

Conn, V. S., Hafdahl, A. R., \& Mehr, D. R. (2011). Interventions to increase physical activity among healthy adults: meta-analysis of outcomes. American Journal of Public Health, 101(4), 751-758. doi:10.2105/ AJPH.2010.194381

Conner, M., \& Norman, P. (2005). Predicting health behaviour: McGraw-Hill Education (UK).

Conner, M. T., Perugini, M., O'Gorman, R., Ayres, K., \& Prestwich, A. (2007). Relations between implicit and explicit measures of attitudes and measures of behavior: Evidence of moderation by individual difference variables. Pers Soc Psychol B, 33(12), 1727-1740. doi:10.1177/0146167207309194

Conroy, D. E., Hyde, A. L., Doerksen, S. E., \& Ribeiro, N. F. (2010). Implicit attitudes and explicit motivation prospectively predict physical activity. Annals of Behavioral Medicine, 39(2), 112-118. doi:10.1007/ s12160-010-9161-0

Cross, A. J., Leitzmann, M. F., Gail, M. H., Hollenbeck, A. R., Schatzkin, A., \& Sinha, R. (2007). A prospective study of red and processed meat intake in relation to cancer risk. PLoS Medicine, 4(12), e325. doi:10.1371/journal.pmed.0040325

Crutzen, R., \& Peters, G.-J. Y. (2017). Scale quality: alpha is an inadequate estimate and factor-analytic evidence is needed first of all. Health Psychology Review, 11(3), 242-247. doi:10.1080/17437199.20 15.1124240

Cunningham, W. A., Preacher, K. J., \& Banaji, M. R. (2001). Implicit attitude measures: Consistency, stability, and convergent validity. Psychological Science, 12(2), 163-170.

Cutler, R. E., \& Storm, T. (1975). Observational study of alcohol consumption in natural settings. The Vancouver beer parlor. Journal of Studies on Alcohol, 36(9), 1173-1183. doi:10.15288/jsa.1975.36.1173

Dal Cin, S., Gibson, B., Zanna, M. P., Shumate, R., \& Fong, G. T. (2007). Smoking in Movies, Implicit Associations of Smoking With the Self, and Intentions to Smoke. Psychological Science, 18(7), 559563. doi:10.1111/j.1467-9280.2007.01939.x

Dalege, J., Borsboom, D., van Harreveld, F., \& van der Maas, H. L. J. (2018). The Attitudinal Entropy (AE) Framework as a General Theory of Individual Attitudes. Psychological inquiry, 29(4), 175-193. doi:1 $0.1080 / 1047840 \times .2018 .1537246$

Dasgupta, N., \& Rivera, L. M. (2008). When Social Context Matters: The Influence of Long-Term Contact and Short-Term Exposure to Admired Outgroup Members on Implicit Attitudes and Behavioral Intentions. Social Cognition, 26(1), 112-123. doi:10.1521/soco.2008.26.1.112

Davis, C. G., Thake, J., \& Vilhena, N. (2010). Social desirability biases in self-reported alcohol consumption and harms. Addictive Behaviors, 35(4), 302-311. doi:https://doi.org/10.1016/j.addbeh.2009.11.001

De Houwer, J. (2003). The extrinsic affective Simon task. Experimental psychology, 50(2), 77.

De Houwer, J. (2006). What are implicit measures and why are we using them. The handbook of implicit cognition and addiction, 11-28.

De Houwer, J., \& De Bruycker, E. (2007). Implicit attitudes towards meat and vegetables in vegetarians and nonvegetarians. International Journal of Psychology, 42(3), 158-165.

De Houwer, J., Thomas, S., \& Baeyens, F. (2001). Association learning of likes and dislikes: A review of 25 years of research on human evaluative conditioning. Psychological Bulletin, 127(6), 853.

De Vries, H. (2017). An integrated approach for understanding health behavior; the I-Change Model as an example. Psychology and Behavioral Science International Journal, 2(2), 555-585.

De Vries, H., Kremers, S., Smeets, T., \& Reubsaet, A. (2008). Clustering of diet, physical activity and smoking and a general willingness to change. Psychology and Health, 23(3), 265-278. doi:10.1080/14768320701349107 
De Vries, H., Mesters, I., Van de Steeg, H., \& Honing, C. (2005). The general public's information needs and perceptions regarding hereditary cancer: an application of the Integrated Change Model. Patient education and counseling, 56(2), 154-165. doi:10.1016/j.pec.2004.01.002

Deforche, B. I., De Bourdeaudhuij, I. M., \& Tanghe, A. P. (2006). Attitude toward physical activity in normal-weight, overweight and obese adolescents. J Adolesc Health, 38(5), 560-568. doi:10.1016/j. jadohealth.2005.01.015

Deluchi, M., Costa, F. S., Friedman, R., Gonçalves, R., \& Bizarro, L. (2017). Attentional bias to unhealthy food in individuals with severe obesity and binge eating. Appetite, 108, 471-476. doi:https://doi. org/10.1016/j.appet.2016.11.012

Dennis, T. A., \& O'Toole, L. J. (2014). Mental health on the go: Effects of a gamified attention-bias modification mobile application in trait-anxious adults. Clinical Psychological Science, 2(5), 576-590.

DeSmet, A., Shegog, R., Van Ryckeghem, D., Crombez, G., \& De Bourdeaudhuij, I. (2015). A systematic review and meta-analysis of interventions for sexual health promotion involving serious digital games. Games for health journal, 4(2), 78-90.

Devos, T. (2008). Implicit attitudes 101: Theoretical and empirical insights. Attitudes and attitude change, 61-84.

Devos, T. (2011). Theoretical and Empirical Insights. Attitudes and attitude change.

Dibb, S., \& Fitzpatrick, I. (2014). Let's talk about meat: changing dietary behaviour for the 21st century: Eating Better.

Dimofte, C. V. (2010). Implicit measures of consumer cognition: A review. Psychology \& Marketing, 27(10), 921-937.

Dovidio, J. F., Kawakami, K., \& Gaertner, S. L. (2002). Implicit and explicit prejudice and interracial interaction. Journal of Personality and Social Psychology, 82(1), 62. doi:10.1037/0022-3514.82.1.62

Dovidio, J. F., Kawakami, K., Johnson, C., Johnson, B., \& Howard, A. (1997). On the nature of prejudice: Automatic and controlled processes. Journal of Experimental Social Psychology, 33(5), 510-540.

Downs, D. S., \& Hausenblas, H. A. (2005). The theories of reasoned action and planned behavior applied to exercise: A meta-analytic update. Journal of Physical Activity and Health, 2(1), 76-97.

Durante, R., \& Ainsworth, B. E. (1996). The recall of physical activity: using a cognitive model of the question-answering process. Medicine \& Science in Sports \& Exercise.

Eagly, A. H., \& Chaiken, S. (1993). The psychology of attitudes: Harcourt Brace Jovanovich College Publishers.

Ebert, I. D., Steffens, M. C., von Stulpnagel, R., \& Jelenec, P. (2009). How to like yourself better, or chocolate less: Changing implicit attitudes with one IAT task. Journal of Experimental Social Psychology, 45(5), 1098-1104. doi:10.1016/j.jesp.2009.06.008

Ebert, I. D., Steffens, M. C., von Stülpnagel, R., \& Jelenec, P. (2009). How to like yourself better, or chocolate less: Changing implicit attitudes with one IAT task. Journal of Experimental Social Psychology, 45(5), 1098-1104. doi:https://doi.org/10.1016/j.jesp.2009.06.008

Endrighi, R., Basen-Engquist, K., Szeto, E., Perkins, H., Baum, G., Cox-Martin, M., . . Waters, A. J. (2016). Self-reported and automatic cognitions are associated with exercise behavior in cancer survivors. Health Psychology, 35(8), 824-828. doi:10.1037/hea0000385

Fazio, R. H. (1995). Attitudes as object-evaluation associations: Determinants, consequences, and correlates of attitude accessibility. Attitude strength: Antecedents and consequences, 4, 247-282.

Fazio, R. H., \& Olson, M. A. (2003). Implicit measures in social cognition research: Their meaning and use. Annual Review of Psychology, 54(1), 297-327.

Fazio, R. H., Sanbonmatsu, D. M., Powell, M. C., \& Kardes, F. R. (1986). On the automatic activation of attitudes. Journal of Personality and Social Psychology, 50(2), 229.

Fenigstein, A., Scheier, M. F., \& Buss, A. H. (1975). Public and private self-consciousness: Assessment and theory. Journal of consulting and clinical psychology, 43(4), 522.

Ferreira de Moraes, A. C., Guerra, P. H., \& Rossi Menezes, P. (2013). The worldwide prevalence of insufficient physical activity in adolescents; a systematic review. Nutrición hospitalaria, 28(3). 
Festinger, L. (1957). A theory of cognitive dissonance. Evanston, Il: Row, Peterson.

Fishbein, M. (1963). An investigation of the relationships between beliefs about an object and the attitude toward that object. Human relations, 16(3), 233-239.

Fishbein, M. (1967). Readings in attitude theory and measurement.

Fishbein, M., \& Ajzen, I. (2011). Predicting and changing behavior: The reasoned action approach: Taylor \& Francis.

FitzGerald, C., \& Hurst, S. (2017). Implicit bias in healthcare professionals: a systematic review. BMC medical ethics, 18(1), 19.

Flycatcher Internet Research. (2018). Flycatcher Internet Research. Retrieved from https://www.flycatcher. eu/en/

French, D. P., Sutton, S., Hennings, S. J., Mitchell, J., Wareham, N. J., Griffin, S., .. Kinmonth, A. L. (2005). The importance of affective beliefs and attitudes in the Theory of Planned Behavior: Predicting intention to increase physical activity. Journal of Applied Social Psychology, 35(9), 1824-1848. doi:10.1111/j.1559-1816.2005.tb02197.x

Friel, S., Dangour, A. D., Garnett, T., Lock, K., Chalabi, Z., Roberts, I., . . McMichael, A. J. (2009). Public health benefits of strategies to reduce greenhouse-gas emissions: food and agriculture. The Lancet, 374(9706), 2016-2025.

Friese, M., Bluemke, M., \& Wanke, M. (2007). Predicting voting behavior with implicit attitude measures: the 2002 German parliamentary election. Exp Psychol, 54(4), 247-255. doi:10.1027/1618-3169.54.4.247

Friese, M., Hofmann, W., \& Wänke, M. (2008). When impulses take over: Moderated predictive validity of explicit and implicit attitude measures in predicting food choice and consumption behaviour. British Journal of Social Psychology, 47(3), 397-419.

Friese, M., Smith, C. T., Plischke, T., Bluemke, M., \& Nosek, B. A. (2012). Do implicit attitudes predict actual voting behavior particularly for undecided voters? PloS one, 7(8), e44130.

Friese, M., Wänke, M., \& Plessner, H. (2006). Implicit consumer preferences and their influence on product choice. Psychology \& Marketing, 23(9), 727-740.

Gawronski, B. (2009). Ten frequently asked questions about implicit measures and their frequently supposed, but not entirely correct answers. Canadian Psychology/Psychologie canadienne, 50(3), 141.

Gawronski, B., \& Bodenhausen, G. V. (2006). Associative and propositional processes in evaluation: an integrative review of implicit and explicit attitude change. Psychological Bulletin, 132(5), 692.

Gawronski, B., \& Bodenhausen, G. V. (2007). Unraveling the Processes Underlying Evaluation: Attitudes from the Perspective of the Ape Model. Social Cognition, 25(5), 687-717. doi:10.1521/soco.2007.25.5.687

Gawronski, B., \& De Houwer, J. (2014). Implicit measures in social and personality psychology. Handbook of research methods in social and personality psychology, 2, 283-310.

Gawronski, B., Hofmann, W., \& Wilbur, C. J. (2006). Are "implicit" attitudes unconscious? Consciousness and cognition, 15(3), 485-499.

Gawronski, B., Morrison, M., Phills, C. E., \& Galdi, S. (2017). Temporal stability of implicit and explicit measures: A longitudinal analysis. Personality and Social Psychology Bulletin, 43(3), 300-312.

Gawronski, B., \& Strack, F. (2004). On the propositional nature of cognitive consistency: Dissonance changes explicit, but not implicit attitudes. Journal of Experimental Social Psychology, 40(4), 535542. doi:https://doi.org/10.1016/j.jesp.2003.10.005

Giovannucci, E., Rimm, E. B., Stampfer, M. J., Colditz, G. A., Ascherio, A., \& Willett, W. C. (1994). Intake of fat, meat, and fiber in relation to risk of colon cancer in men. Cancer Research, 54(9), 2390-2397.

Glasman, L. R., \& Albarracin, D. (2006). Forming attitudes that predict future behavior: A meta-analysis of the attitude-behavior relation. Psychological Bulletin, 132(5), 778-822. doi:10.1037/00332909.132.5.778

Godin, G. (1994). Theories of reasoned action and planned behavior: usefulness for exercise promotion. Medicine \& Science in Sports \& Exercise. 
Godin, G., Bélanger-Gravel, A., Eccles, M., \& Grimshaw, J. (2008). Healthcare professionals' intentions and behaviours: A systematic review of studies based on social cognitive theories. Implementation Science, 3(1), 36.

Godin, G., Valois, P., Lepage, L., \& Desharnais, R. (1992). Predictors of smoking behaviour: an application of Ajzen's theory of planned behaviour. British journal of addiction, 87(9), 1335-1343.

Goldstein, S. P., Forman, E. M., Meiran, N., Herbert, J. D., Juarascio, A. S., \& Butryn, M. L. (2014). The discrepancy between implicit and explicit attitudes in predicting disinhibited eating. Eating Behaviors, 15(1), 164-170. doi:10.1016/j.eatbeh.2013.10.021

Gollwitzer, M., Banse, R., Eisenbach, K., \& Naumann, A. (2007). Effectiveness of the Vienna social competence training on explicit and implicit aggression. European Journal of Psychological Assessment, 23(3), 150-156.

Gotlib, I. H., Krasnoperova, E., Yue, D. N., \& Joormann, J. (2004). Attentional Biases for Negative Interpersonal Stimuli in Clinical Depression. Journal of Abnormal Psychology, 113(1), 127-135. doi:10.1037/0021843X.113.1.121

Govan, C. L., \& Williams, K. D. (2004). Reversing or eliminating IAT effects by changing the affective valence of the stimulus items. Journal of Experimental Social Psychology, 40(3), 357-365.

Graça, J., Calheiros, M. M., \& Oliveira, A. (2015). Attached to meat? (Un)Willingness and intentions to adopt a more plant-based diet. Appetite, 95, 113-125.

Graesser, A., Chipman, P., Leeming, F., \& Biedenbach, S. (2009). Serious Games: Mechanisms and Effects.

Graham, D. J., Sirard, J. R., \& Neumark-Sztainer, D. (2011). Adolescents' attitudes toward sports, exercise, and fitness predict physical activity 5 and 10 years later. Preventive Medicine, 52(2), 130-132. doi:http://dx.doi.org/10.1016/j.ypmed.2010.11.013

Green, A. R., Carney, D. R., Pallin, D. J., Ngo, L. H., Raymond, K. L., lezzoni, L. I., \& Banaji, M. R. (2007). Implicit bias among physicians and its prediction of thrombolysis decisions for black and white patients. Journal of general internal medicine, 22(9), 1231-1238.

Greenwald, A. G., \& Banaji, R. (1995). Implicit social cognition: attitudes, self-esteem, and stereotypes. Psychological review, 102(1), 4.

Greenwald, A. G., McGhee, D. E., \& Schwartz, J. L. (1998). Measuring individual differences in implicit cognition: the implicit association test. Journal of Personality and Social Psychology, 74(6), 14641480. doi:10.1037/0022-3514.74.6.1464

Greenwald, A. G., Nosek, B. A., \& Banaji, M. R. (2003). Understanding and using the implicit association test: I. An improved scoring algorithm. Journal of Personality and Social Psychology, 85(2), 197. doi:10.1037/0022-3514.85.2.197

Greenwald, A. G., Poehlman, T. A., Uhlmann, E. L., \& Banaji, M. R. (2009). Understanding and Using the Implicit Association Test: III. Meta-Analysis of Predictive Validity. Journal of Personality and Social Psychology, 97(1), 17-41. doi:10.1037/a0015575

Grønhøj, A., Bech-Larsen, T., Chan, K., \& Tsang, L. (2012). Using theory of planned behavior to predict healthy eating among Danish adolescents. Health Education, 113(1), 4-17.

Grumm, M., Nestler, S., \& von Collani, G. (2009). Changing explicit and implicit attitudes: The case of selfesteem. Journal of Experimental Social Psychology, 45(2), 327-335. doi:10.1016/j.jesp.2008.10.006

Gschwendner, T., Hofmann, W., \& Schmitt, M. (2006). Synergistic moderator effects of situation and person factors of awareness and adjustment on the consistency of implicit and explicit attitudes. Journal of Individual Differences, 27(1), 47-56.

Haddock, G., Foad, C., Windsor-Shellard, B., Dummel, S., \& Adarves-Yorno, I. (2017). On the attitudinal consequences of being mindful: Links between mindfulness and attitudinal ambivalence. Personality and Social Psychology Bulletin, 43(4), 439-452.

Haddock, G., \& Maio, G. R. (2004). Contemporary perspectives on the psychology of attitudes: Psychology Press.

Hagger, M., \& Chatzisarantis, N. L. (2014). An integrated behavior change model for physical activity. Exercise and Sport Sciences Reviews, 42(2), 62-69. 
Hagger, M., Chatzisarantis, N. L. D., \& Biddle, S. J. H. (2002). A meta-analytic review of the theories of reasoned action and planned behavior in physical activity: Predictive validity and the contribution of additional variables. Journal of Sport and Exercise Psychology, 24(1), 3-32. doi:10.1123/jsep.24.1.3

Hahn, A., \& Gawronski, B. (2014). Do implicit evaluations reflect unconscious attitudes? Behavioral and Brain Sciences, 37(1), 28-29.

Hahn, A., Judd, C. M., Hirsh, H. K., \& Blair, I. V. (2014). Awareness of implicit attitudes. Journal of Experimental Psychology: General, 143(3), 1369.

Hajcak, G., \& Foti, D. (2008). Errors Are Aversive:Defensive Motivation and the Error-Related Negativity. Psychological Science, 19(2), 103-108. doi:10.1111/j.1467-9280.2008.02053.x

Hallal, P. C., Andersen, L. B., Bull, F. C., Guthold, R., Haskell, W., Ekelund, U., \& Lancet Physical Activity Series Working, G. (2012). Global physical activity levels: surveillance progress, pitfalls, and prospects. Lancet, 380(9838), 247-257. doi:10.1016/S0140-6736(12)60646-1

Hausenblas, H. A., Carron, A. V., \& Mack, D. E. (1997). Application of the theories of reasoned action and planned behavior to exercise behavior: A meta-analysis. Journal of Sport and Exercise Psychology, 19(1), 36-51.

Hayes, S. C., Luoma, J. B., Bond, F. W., Masuda, A., \& Lillis, J. (2006). Acceptance and Commitment Therapy: Model, processes and outcomes. Behaviour Research and Therapy, 44(1), 1-25. doi:https://doi. org/10.1016/j.brat.2005.06.006

Health Council of the Netherlands. (2017). Physical activity guidelines 2017. Retrieved from https://www. healthcouncil.nl/documents/advisory-reports/2017/08/22/physical-activity-guidelines-2017

Health, U. D. o., \& Services, H. (2008). 2008 Physical Activity Guidelines for Americans.

Hebert, J. R., Clemow, L., Pbert, L., Ockene, I. S., \& Ockene, J. K. (1995). Social desirability bias in dietary self-report may compromise the validity of dietary intake measures. International journal of epidemiology, 24(2), 389-398.

Hebert, J. R., Ma, Y., Clemow, L., Ockene, I. S., Saperia, G., Stanek III, E. J., . . Ockene, J. K. (1997). Gender differences in social desirability and social approval bias in dietary self-report. American Journal of Epidemiology, 146(12), 1046-1055.

Hoffman, K. M., Trawalter, S., Axt, J. R., \& Oliver, M. N. (2016). Racial bias in pain assessment and treatment recommendations, and false beliefs about biological differences between blacks and whites. Proceedings of the National Academy of Sciences of the United States of America, 113(16), 42964301. doi:10.1073/pnas.1516047113

Hofmann, W., De Houwer, J., Perugini, M., Baeyens, F., \& Crombez, G. (2010). Evaluative conditioning in humans: a meta-analysis. Psychological Bulletin, 136(3), 390.

Hofmann, W., Friese, M., \& Roefs, A. (2009). Three ways to resist temptation: The independent contributions of executive attention, inhibitory control, and affect regulation to the impulse control of eating behavior. Journal of Experimental Social Psychology, 45(2), 431-435.

Hofmann, W., Friese, M., \& Wiers, R. W. (2008). Impulsive versus reflective influences on health behavior: A theoretical framework and empirical review. Health Psychology Review, 2(2), 111-137. doi:10.1080/17437190802617668

Hofmann, W., Gschwendner, T., Nosek, B. A., \& Schmitt, M. (2005). What moderates implicit-explicit consistency? European Review of Social Psychology, 16(1), 335-390. doi:10.1080/10463280500443228

Hofmann, W., Gschwendner, T., \& Schmitt, M. (2005). On implicit-explicit consistency: The moderating role of individual differences in awareness and adjustment. European Journal of Personality: Published for the European Association of Personality Psychology, 19(1), 25-49.

Hofmann, W., Rauch, W., \& Gawronski, B. (2007). And deplete us not into temptation: Automatic attitudes, dietary restraint, and self-regulatory resources as determinants of eating behavior. Journal of Experimental Social Psychology, 43(3), 497-504. doi:10.1016/j.jesp.2006.05.004

Hollands, G. J., Prestwich, A., \& Marteau, T. M. (2011). Using aversive images to enhance healthy food choices and implicit attitudes: An experimental test of evaluative conditioning. Health Psychology, 30(2), 195-203. doi:10.1037/a0022261 
Hölzel, B. K., Lazar, S. W., Gard, T., Schuman-Olivier, Z., Vago, D. R., \& Ott, U. (2011). How Does Mindfulness Meditation Work? Proposing Mechanisms of Action From a Conceptual and Neural Perspective. Perspectives on Psychological Science, 6(6), 537-559. doi:10.1177/1745691611419671

Houben, K., Nederkoorn, C., Wiers, R. W., \& Jansen, A. (2011). Resisting temptation: decreasing alcoholrelated affect and drinking behavior by training response inhibition. Drug and Alcohol Dependence, 116(1-3), 132-136. doi:10.1016/j.drugalcdep.2010.12.011

Huijding, J., de Jong, P. J., Wiers, R. W., \& Verkooijen, K. (2005). Implicit and explicit attitudes toward smoking in a smoking and a nonsmoking setting. Addictive Behaviors, 30(5), 949-961. doi:https:// doi.org/10.1016/j.addbeh.2004.09.014

Hyde, A. L., Doerksen, S. E., Ribeiro, N. F., \& Conroy, D. E. (2010). The independence of implicit and explicit attitudes toward physical activity: Introspective access and attitudinal concordance. Psychology of Sport and Exercise, 11(5), 387-393. doi:10.1016/j.psychsport.2010.04.008

Hyde, A. L., Elavsky, S., Doerksen, S. E., \& Conroy, D. E. (2012). The stability of automatic evaluations of physical activity and their relations with physical activity. Journal of Sport and Exercise Psychology, 34(6), 715-736.

Inquisit. (2015). (Version 4.0). Seattle, WA: Millisecond Software.

Jakobsen, J. C., Gluud, C., Wetterslev, J., \& Winkel, P. (2017). When and how should multiple imputation be used for handling missing data in randomised clinical trials-a practical guide with flowcharts. BMC medical research methodology, 17(1), 162.

Janis, I. L., \& King, B. T. (1954). The influence of role playing on opinion change. The journal of abnormal and social psychology, 49(2), 211.

Johnston, J., Prynne, C., Stephen, A., \& Wadsworth, M. (2007). Haem and non-haem iron intake through 17 years of adult life of a British Birth Cohort. British Journal of Nutrition, 98(5), 1021-1028.

Jones, C. R., Olson, M. A., \& Fazio, R. H. (2010). Evaluative conditioning: The "how" question Advances in experimental social psychology (Vol. 43, pp. 205-255): Elsevier.

Juraskova, I., O'Brien, M., Mullan, B., Bari, R., Laidsaar-Powell, R., \& McCaffery, K. (2012). HPV vaccination and the effect of information framing on intentions and behaviour: An application of the theory of planned behaviour and moral norm. International journal of behavioral medicine, 19(4), 518-525.

Kabat-Zinn, J. (2003). Mindfulness-Based Interventions in Context: Past, Present, and Future. Clinical Psychology: Science and Practice, 10(2), 144-156. doi:doi:10.1093/clipsy.bpg016

Karpen, S. C., Jia, L., \& Rydell, R. J. (2012). Discrepancies between implicit and explicit attitude measures as an indicator of attitude strength. European Journal of Social Psychology, 42(1), 24-29.

Karpinski, A., \& Steinman, R. B. (2006). The single category implicit association test as a measure of implicit social cognition. Journal of Personality and Social Psychology, 91(1), 16-32. doi:10.1037/00223514.91.1.16

Karpinski, A., Steinman, R. B., \& Hilton, J. L. (2005). Attitude importance as a moderator of the relationship between implicit and explicit attitude measures. Personality and Social Psychology Bulletin, 31(7), 949-962.

Kelemen, L. E., Kushi, L. H., Jacobs, D. R., \& Cerhan, J. R. (2005). Associations of dietary protein with disease and mortality in a prospective study of postmenopausal women. American Journal of Epidemiology, 161(3), 239-249.

Knittle, K., Nurmi, J., Crutzen, R., Hankonen, N., Beattie, M., \& Dombrowski, S. U. (2018). How can interventions increase motivation for physical activity? A systematic review and meta-analysis. Health Psychology Review, 1-20. doi:10.1080/17437199.2018.1435299

Kontogianni, M. D., Panagiotakos, D. B., Pitsavos, C., Chrysohoou, C., \& Stefanadis, C. (2008). Relationship between meat intake and the development of acute coronary syndromes: the CARDIO2000 casecontrol study. European Journal of Clinical Nutrition, 62(2), 171-177. doi:10.1038/sj.ejcn.1602713

Koole, S. L., Dijksterhuis, A., \& Van Knippenberg, A. (2001). What's in a name: Implicit self-esteem and the automatic self. Journal of Personality and Social Psychology, 80(4), 669. 
Koole, S. L., Govorun, O., Cheng, C. M., \& Gallucci, M. (2009). Pulling yourself together: Meditation promotes congruence between implicit and explicit self-esteem. Journal of Experimental Social Psychology, 45(6), 1220-1226.

Kraus, S. J. (1995). Attitudes and the Prediction of Behavior: A Meta-Analysis of the Empirical Literature. Personality and Social Psychology Bulletin, 21(1), 58-75. doi:10.1177/0146167295211007

Krickel, B. (2018). Are the states underlying implicit biases unconscious?-A Neo-Freudian answer. Philosophical Psychology, 31(7), 1007-1026.

Lea, E., \& Worsley, A. (2001). Influences on meat consumption in Australia. Appetite, 36(2), 127-136.

Levine, D. S., \& Strube, M. J. (2012). Environmental Attitudes, Knowledge, Intentions and Behaviors Among College Students. The Journal of Social Psychology, 152(3), 308-326. doi:10.1080/00224545.2011.6 04363

Locke, E. A., \& Latham, G. P. (1990). A theory of goal setting \& task performance: Prentice-Hall, Inc.

Lowenthal, K. M. (2001). An introduction to psychological tets and scales. Philadelphia: Psychology Press.

Lugtig, P. (2014). Panel Attrition: Separating Stayers, Fast Attriters, Gradual Attriters, and Lurkers. Sociological Methods \& Research, 43(4), 699-723. doi:10.1177/0049124113520305

MacKillop, J., \& Anderson, E. J. (2007). Further psychometric validation of the mindful attention awareness scale (MAAS). Journal of Psychopathology and Behavioral Assessment, 29(4), 289-293.

MacLeod, C., Mathews, A., \& Tata, P. (1986). Attentional bias in emotional disorders. Journal of Abnormal Psychology, 95(1), 15-20. doi:10.1037/0021-843X.95.1.15

Maison, D., Greenwald, A. G., \& Bruin, R. (2001). The Implicit Association Test as a measure of implicit consumer attitudes.

Maison, D., Greenwald, A. G., \& Bruin, R. H. (2004). Predictive validity of the Implicit Association Test in studies of brands, consumer attitudes, and behavior. Journal of consumer psychology, 14(4), 405415.

Maliszewski, N. (2011). How Do People Resolve Conflict Between Implicit and Explicit Attitudes? Polish Psychological Bulletin, 42(1), 36-45.

Markland, D., Hall, C. R., Duncan, L. R., \& Simatovic, J. (2015). The effects of an imagery intervention on implicit and explicit exercise attitudes. Psychology of Sport and Exercise, 17, 24-31. doi:http://dx.doi. org/10.1016/j.psychsport.2014.11.007

Marlatt, G. A. (2002). Buddhist philosophy and the treatment of addictive behavior. Cognitive and behavioral practice, 9(1), 44-50.

Marmot, M., Atinmo, T., Byers, T., Chen, J., Hirohata, T., Jackson, A., .. Leitzmann, C. (2007). Food, nutrition, physical activity, and the prevention of cancer: a global perspective.

Mattavelli, S., Avishai, A., Perugini, M., Richetin, J., \& Sheeran, P. (2017). How can implicit and explicit attitudes both be changed? Testing two interventions to promote consumption of green vegetables. Annals of Behavioral Medicine, 51(4), 511-518.

Maynard, O. M., Leonards, U., Attwood, A. S., Bauld, L., Hogarth, L., \& Munafò, M. R. (2015). Effects of first exposure to plain cigarette packaging on smoking behaviour and attitudes: a randomised controlled study. BMC public health, 15(1), 240.

McCarthy, D. M., \& Thompsen, D. M. (2006). Implicit and explicit measures of alcohol and smoking cognitions. Psychology of Addictive Behaviors, 20(4), 436-444. doi:10.1037/0893-164X.20.4.436

McConnell, A. R., Dunn, E. W., Austin, S. N., \& Rawn, C. D. (2011). Blind spots in the search for happiness: Implicit attitudes and nonverbal leakage predict affective forecasting errors. Journal of Experimental Social Psychology, 47(3), 628-634.

McConnell, A. R., \& Leibold, J. M. (2001). Relations among the Implicit Association Test, Discriminatory Behavior, and Explicit Measures of Racial Attitudes. Journal of Experimental Social Psychology, 37(5), 435-442. doi:10.1006/jesp.2000.1470

McDonald, R. P. (2013). Test theory: A unified treatment: Psychology Press. 
McEachan, R. R. C., Conner, M., Taylor, N. J., \& Lawton, R. J. (2011). Prospective prediction of health-related behaviours with the Theory of Planned Behaviour: a meta-analysis. Health Psychology Review, 5(2), 97-144. doi:10.1080/17437199.2010.521684

McEachan, R. R. C., Taylor, N., Harrison, R., Lawton, R., Gardner, P., \& Conner, M. (2016). Meta-Analysis of the Reasoned Action Approach (RAA) to Understanding Health Behaviors. Annals of Behavioral Medicine, 50(4), 592-612. doi:10.1007/s12160-016-9798-4

McGuire, S. (2016). World Cancer Report 2014. Geneva, Switzerland: World Health Organization, International Agency for Research on Cancer, WHO Press, 2015. Advances in Nutrition, 7(2), 418419. doi:10.3945/an.116.012211

Millar, M. G. (2011). Predicting Dental Flossing Behavior: The Role of Implicit and Explicit Responses and Beliefs. Basic and Applied Social Psychology, 33(1), 7-15. doi:10.1080/01973533.2010.539949

Miller, L. C., Murphy, R., \& Buss, A. H. (1981). Consciousness of body: Private and public. Journal of Personality and Social Psychology, 41(2), 397.

Mitchell, J. P., Nosek, B. A., \& Banaji, M. R. (2003). Contextual variations in implicit evaluation. Journal of Experimental Psychology: General, 132(3), 455.

Mogg, K., Bradley, B. P., Field, M., \& De Houwer, J. (2003). Eye movements to smoking-related pictures in smokers: relationship between attentional biases and implicit and explicit measures of stimulus valence. Addiction, 98(6), 825-836.

Murphy, C., \& MacKillop, J. (2012). Living in the here and now: interrelationships between impulsivity, mindfulness, and alcohol misuse. Psychopharmacology, 219(2), 527-536. doi:10.1007/s00213-0112573-0

Muschalik, C., Elfeddali, I., Candel, M. J. J. M., Crutzen, R., \& de Vries, H. (2019). Does the discrepancy between implicit and explicit attitudes moderate the relationships between explicit attitude and (intention to) being physically active? BMC Psychology, 7(1), 52. doi:10.1186/s40359-019-0322-z

Muschalik, C., Elfeddali, I., Candel, M. J. J. M., \& de Vries, H. (2018). A longitudinal study on how implicit attitudes and explicit cognitions synergistically influence physical activity intention and behavior. BMC Psychology, 6(1), 18. doi:10.1186/s40359-018-0229-0

Muthén, L. K., \& Muthén, B. O. (2012). MPlus: statistical analysis with latent variables--User's guide.

Neff, K. (2003a). Self-compassion: An alternative conceptualization of a healthy attitude toward oneself. Self and identity, 2(2), 85-101.

Neff, K. D. (2003b). The development and validation of a scale to measure self-compassion. Self and identity, 2(3), 223-250.

Neumann, R., Hülsenbeck, K., \& Seibt, B. (2004). Attitudes towards people with AIDS and avoidance behavior: Automatic and reflective bases of behavior. Journal of Experimental Social Psychology, 40(4), 543-550. doi:10.1016/j.jesp.2003.10.006

Newton, N. C., Teesson, M., Vogl, L. E., \& Andrews, G. (2010). Internet-based prevention for alcohol and cannabis use: final results of the Climate Schools course. Addiction, 105(4), 749-759.

Norman, P., Conner, M., \& Bell, R. (1999). The theory of planned behavior and smoking cessation. Health Psychology, 18(1), 89.

Nosek, B. A. (2005). Moderators of the relationship between implicit and explicit evaluation. J Exp Psychol Gen, 134(4), 565-584. doi:10.1037/0096-3445.134.4.565

Nosek, B. A., \& Banaji, M. R. (2001). The go/no-go association task. Social cognition, 19(6), 625-666.

Nosek, B. A., \& Banaji, M. R. (2016). (At least) two factors moderate the relationship between implicit and explicit attitudes.

Nosek, B. A., \& Greenwald, A. G. (2009). (Part of) the case for a pragmatic approach to validity: Comment on De Houwer, Teige-Mocigemba, Spruyt, and Moors (2009).

Nosek, B. A., Hawkins, C. B., \& Frazier, R. S. (2011). Implicit social cognition: From measures to mechanisms. Trends in cognitive sciences, 15(4), 152-159.

Nunnally, J. C., \& Bernstein, I. H. (1994). Psychometric Theory. New York: McGraw-Hill. 
Olson, A. L., Gaffney, C. A., Starr, P., \& Dietrich, A. J. (2007). The impact of an appearance-based educational intervention on adolescent intention to use sunscreen. Health Education Research, 23(5), 763-769.

Olson, M. A., \& Fazio, R. H. (2003). Relations between implicit measures of prejudice: What are we measuring? Psychological Science, 14(6), 636-639.

Olson, M. A., \& Fazio, R. H. (2006). Reducing automatically activated racial prejudice through implicit evaluative conditioning. Personality and Social Psychology Bulletin, 32(4), 421-433.

Olson, M. A., Fazio, R. H., \& Hermann, A. D. (2007). Reporting tendencies underlie discrepancies between implicit and explicit measures of self-esteem. Psychological Science, 18(4), 287-291.

Osterlind, S. J., Tabachnick, B. G., \& Fidell, L. S. (2001). SPSS for Window Workbook to Acompany: Using Multivariate Statistics.--4th.--Tabachnick and Fidell: Allyn and bacon.

Øygard, L., \& Rise, J. (1996). Predicting the intention to eat healthier food among young adults. Health Education Research, 11(4), 453-461.

Palfai, T. P., \& Ostafin, B. D. (2003). Alcohol-related motivational tendencies in hazardous drinkers: assessing implicit response tendencies using the modified-IAT. Behaviour research and therapy, 41(10), 1149-1162.

Pan, A., Sun, Q., Bernstein, A. M., Schulze, M. B., Manson, J. E., Stampfer, M. J., .. Hu, F. B. (2012). Red meat consumption and mortality: results from 2 prospective cohort studies. Archives of internal medicine, 172(7), 555-563.

Pan, A., Sun, Q., Bernstein, A. M., Schulze, M. B., Manson, J. E., Willett, W. C., \& Hu, F. B. (2011). Red meat consumption and risk of type 2 diabetes: 3 cohorts of US adults and an updated meta-analysis. American Journal of Clinical Nutrition, 94(4), 1088-1096. doi:10.3945/ajcn.111.018978

Panzone, L., Hilton, D., Sale, L., \& Cohen, D. (2016). Socio-demographics, implicit attitudes, explicit attitudes, and sustainable consumption in supermarket shopping. Journal of Economic Psychology, 55, 77-95. doi:https://doi.org/10.1016/j.joep.2016.02.004

Park, H., \& Kim, N. (2008). Predicting factors of physical activity in adolescents: a systematic review. Asian Nursing Research, 2(2), 113-128. doi:10.1016/S1976-1317(08)60035-3

Patch, C. S., Tapsell, L. C., \& Williams, P. G. (2005). Attitudes and intentions toward purchasing novel foods enriched with omega-3 fatty acids. Journal of nutrition education and behavior, 37(5), 235-241.

Patton, J. H., Stanford, M. S., \& Barratt, E. S. (1995). Factor structure of the Barratt impulsiveness scale. Journal of clinical psychology, 51(6), 768-774.

Perugini, M. (2005). Predictive models of implicit and explicit attitudes. British Journal of Social Psychology, 44(Pt 1), 29-45. doi:10.1348/014466604X23491

Peters, G.-J. Y., Abraham, C., \& Crutzen, R. (2012). Full disclosure: Doing behavioural science necessitates sharing. . The European Health Psychologist, 14(2), 77-84.

Peters, G.-J. Y., \& Crutzen, R. (2017). Pragmatic nihilism: how a Theory of Nothing can help health psychology progress. Health Psychology Review, 11(2), 103-121.

Peters, J. R., Erisman, S. M., Upton, B. T., Baer, R. A., \& Roemer, L. (2011). A preliminary investigation of the relationships between dispositional mindfulness and impulsivity. Mindfulness, 2(4), 228-235.

Petty, R. E., Briñol, P., \& DeMarree, K. G. (2007). The Meta-Cognitive Model (MCM) of attitudes: implications for attitude measurement, change, and strength. Social Cognition, 25(5), 657-686.

Petty, R. E., Tormala, Z. L., Brinol, P., \& Jarvis, W. B. G. (2006). Implicit ambivalence from attitude change: an exploration of the PAST model. Journal of Personality and Social Psychology, 90(1), 21.

Prestwich, A., Hurling, R., \& Baker, S. (2011). Implicit shopping: Attitudinal determinants of the purchasing of healthy and unhealthy foods. Psychology \& health, 26(7), 875-885. doi:10.1080/08870446.2010 .509797

Prince, S. A., Adamo, K. B., Hamel, M. E., Hardt, J., Connor Gorber, S., \& Tremblay, M. (2008). A comparison of direct versus self-report measures for assessing physical activity in adults: a systematic review. International Journal of Behavioral Nutrition and Physical Activity, 5(1), 56. doi:10.1186/1479-5868$5-56$ 
Prochaska, J. O., \& Velicer, W. F. (1997). The transtheoretical model of health behavior change. American Journal of Health Promotion, 12(1), 38-48.

R Core Team. (2016). R: A language and environment for statistical computing. R Foundation for Statistical Computing, Vienna, Austria. 2014.

Raes, F., Dewulf, D., Van Heeringen, C., \& Williams, J. M. G. (2009). Mindfulness and reduced cognitive reactivity to sad mood: Evidence from a correlational study and a non-randomized waiting list controlled study. Behaviour Research and Therapy, 47(7), 623-627. doi:https://doi.org/10.1016/j. brat.2009.03.007

Raghunathan, T. E., Lepkowski, J. M., Van Hoewyk, J., \& Solenberger, P. (2001). A multivariate technique for multiply imputing missing values using a sequence of regression models. Survey methodology, 27(1), 85-96.

Rebar, A. L., Dimmock, J. A., Jackson, B., Rhodes, R. E., Kates, A., Starling, J., \& Vandelanotte, C. (2016). A systematic review of the effects of non-conscious regulatory processes in physical activity. Health Psychology Review, 10(4), 395-407. doi:10.1080/17437199.2016.1183505

Rebar, A. L., Ram, N., \& Conroy, D. E. (2015). Using the EZ-diffusion model to score a Single-Category Implicit Association Test of physical activity. Psychology of Sport and Exercise, 16, 96-105. doi:https:// doi.org/10.1016/j.psychsport.2014.09.008

Research Now. (2018). Research Now. Retrieved from https://www.researchnow.com/?lang=gb

Reynolds, K. D., Killen, J. D., Bryson, S. W., Maron, D. J., Taylor, C. B., Maccoby, N., \& Farquhar, J. W. (1990). Psychosocial predictors of physical activity in adolescents. Preventive Medicine, 19(5), 541-551. doi:https://doi.org/10.1016/0091-7435(90)90052-L

Rezai, G., Teng, P. K., Mohamed, Z., \& Shamsudin, M. N. (2012). Consumers' awareness and consumption intention towards green foods. African Journal of Business Management, 6(12), 4496.

Richetin, J., Perugini, M., Prestwich, A., \& O'Gorman, R. (2007). The IAT as a predictor of food choice: The case of fruits versus snacks. International Journal of Psychology, 42(3), 166-173. doi:doi:10.1080/00207590601067078

Rinck, M., \& Becker, E. S. (2007). Approach and avoidance in fear of spiders. Journal of behavior therapy and experimental psychiatry, 38(2), 105-120.

RIVM. (2005). Lokale en nationale Monitor gezondheid. Indicatoren voor de Monitor Volksgezondheid [Local and national health monitoring. Indicators for public health monitoring]. Retrieved from https://www.monitorgezondheid.nl/volksindicatoren.aspx

Roese, N. J., \& Olson, J. M. (1994). Attitude importance as a function of repeated attitude expression. Journal of Experimental Social Psychology, 30(1), 39-51.

Rooke, S. E., Hine, D. W., \& Thorsteinsson, E. B. (2008). Implicit cognition and substance use: A metaanalysis. Addictive Behaviors, 33(10), 1314-1328. doi:https://doi.org/10.1016/j.addbeh.2008.06.009

Rosenberg, M. (1965). Rosenberg self-esteem scale (SES). Society and the adolescent self-image.

Rudman, L. A. (2004). Sources of implicit attitudes. Current Directions in Psychological Science, 13(2), 79-82.

Rydell, R. J., \& McConnell, A. R. (2006). Understanding implicit and explicit attitude change: a systems of reasoning analysis. Journal of Personality and Social Psychology, 91(6), 995-1008. doi:10.1037/00223514.91.6.995

Rydell, R. J., McConnell, A. R., \& Mackie, D. M. (2008). Consequences of discrepant explicit and implicit attitudes: Cognitive dissonance and increased information processing. Journal of Experimental Social Psychology, 44(6), 1526-1532. doi:10.1016/j.jesp.2008.07.006

Rydell, R. J., McConnell, A. R., Mackie, D. M., \& Strain, L. M. (2006). Of two minds: Forming and changing valence-inconsistent implicit and explicit attitudes. Psychological Science, 17(11), 954-958.

SalehNia, B., Mizany, M., Sajadi, S. N., \& Rahimizadeh, M. (2012). A comparison between attitudes of active and inactive students toward sport and physical activities. Procedia - Social and Behavioral Sciences, 31(Supplement C), 61-65. doi:https://doi.org/10.1016/j.sbspro.2011.12.017

Sallis, J. F., Haskell, W. L., Fortmann, S. P., Vranizan, K. M., Taylor, C. B., \& Solomon, D. S. (1986). Predictors of adoption and maintenance of physical activity in a community sample. Preventive Medicine, 15(4), 331-341. doi:10.1016/0091-7435(86)90001-0 
Samuelson, G. (2004). Global strategy on diet, physical activity and health: Taylor \& Francis.

Schröder-Abé, M., Rudolph, A., \& Schütz, A. (2007). High implicit self-esteem is not necessarily advantageous: Discrepancies between explicit and implicit self-esteem and their relationship with anger expression and psychological health. European Journal of Personality: Published for the European Association of Personality Psychology, 21(3), 319-339.

Schulz, D. N., Kremers, S. P., Vandelanotte, C., Van Adrichem, M. J., Schneider, F., Candel, M. J., \& De Vries, H. (2014). Effects of a web-based tailored multiple-lifestyle intervention for adults: a twoyear randomized controlled trial comparing sequential and simultaneous delivery modes. Journal of medical Internet research, 16(1), e26. doi:10.2196/jmir.3094

Schulz, D. N., Schneider, F., De Vries, H., van Osch, L. A., van Nierop, P. W., \& Kremers, S. P. (2012). Program completion of a web-based tailored lifestyle intervention for adults: differences between a sequential and a simultaneous approach. Journal of medical Internet research, 14(2).

Scott, P. (2017). Global panel on agriculture and food systems for nutrition: food systems and diets: facing the challenges of the 21st century. Food Security: The Science, Sociology and Economics of Food Production and Access to Food, 9(3), 653-654.

Shapiro, S. L., Oman, D., Thoresen, C. E., Plante, T. G., \& Flinders, T. (2008). Cultivating mindfulness: effects on well-being. Journal of Clinical Psychology, 64(7), 840-862. doi:10.1002/jclp.20491

Sheeran, P. (2002). Intention-behavior relations: a conceptual and empirical review. European review of social psychology, 12(1), 1-36.

Sheeran, P., Gollwitzer, P. M., \& Bargh, J. A. (2013). Nonconscious processes and health. Health Psychology, 32(5), 460-473. doi:10.1037/a0029203

Sheeran, P., Klein, W. M., \& Rothman, A. J. (2017). Health Behavior Change: Moving from Observation to Intervention. Annual Review of Psychology, 68, 573-600. doi:10.1146/annurev-psych-010416-044007

Sheeran, P., Norman, P., \& Orbell, S. (1999). Evidence that intentions based on attitudes better predict behaviour than intentions based on subjective norms. European Journal of Social Psychology, 29(23), 403-406. doi:Doi 10.1002/(Sici)1099-0992(199903/05)29:2/3<403::Aid-Ejsp942>3.3.Co;2-1

Sheeran, P., \& Orbell, S. (2000). Self-schemas and the theory of planned behaviour. European Journal of Social Psychology, 30(4), 533-550. doi:doi:10.1002/1099-0992(200007/08)30:4<533::AIDEJSP6>3.0.CO;2-F

Sheeran, P., \& Webb, T. L. (2016). The intention-behavior gap. Social and Personality Psychology Compass, 10(9), 503-518.

Siegel, R. (2018). Starbucks's bias training finally happened. Here's what it looked like. Retrieved from https://www.washingtonpost.com/news/business/wp/2018/05/29/starbucks-training-finallyhappened-heres-what-it-looked-like/?noredirect $=$ on\&utm_term $=$. df02f3ac168f

Sinha, R., Cross, A. J., Graubard, B. I., Leitzmann, M. F., \& Schatzkin, A. (2009). Meat intake and mortality: A prospective study of over half a million people. Archives of internal medicine, 169(6), 562-571. doi:10.1001/archinternmed.2009.6

Sloman, S. A. (1996). The empirical case for two systems of reasoning. Psychological Bulletin, 119(1), 3.

Slootmaker, S. M., Schuit, A. J., Chinapaw, M. J., Seidell, J. C., \& van Mechelen, W. (2009). Disagreement in physical activity assessed by accelerometer and self-report in subgroups of age, gender, education and weight status. International Journal of Behavioral Nutrition and Physical Activity, 6(1), 17. doi:10.1186/1479-5868-6-17

Smeets, T., Brug, J., \& De Vries, H. (2006). Effects of tailoring health messages on physical activity. Health Education Research, 23(3), 402-413.

Smeets, T., Kremers, S. P., Brug, J., \& De Vries, H. (2007). Effects of tailored feedback on multiple health behaviors. Annals of Behavioral Medicine, 33(2), 117-123. doi:10.1080/08836610701307801

Song, Y., Manson, J. E., Buring, J. E., \& Liu, S. (2004). A prospective study of red meat consumption and type 2 diabetes in middle-aged and elderly women: the women's health study. Diabetes Care, 27(9), 2108-2115. 
Spalding, L. R., \& Hardin, C. D. (1999). Unconscious unease and self-handicapping: Behavioral consequences of individual differences in implicit and explicit self-esteem. Psychological Science, 10(6), 535-539.

Springvloet, L., Lechner, L., Candel, M. J. J. M., De Vries, H., \& Oenema, A. (2016). Exploring individual cognitions, self-regulation skills, and environmental-level factors as mediating variables of two versions of a Web-based computer-tailored nutrition education intervention aimed at adults: A randomized controlled trial. Appetite, 98(Supplement C), 101-114. doi:https://doi.org/10.1016/j. appet.2015.12.013

Stajkovic, A. D., \& Luthans, F. (1998). Self-efficacy and work-related performance: A meta-analysis. Psychological Bulletin, 124(2), 240.

Steffens, M. C., \& Konig, S. S. (2006). Predicting spontaneous big five behavior with implicit association tests. European Journal of Psychological Assessment, 22(1), 13-20. doi:10.1027/1015-5759.22.1.13

Steinemann, N., Grize, L., Ziesemer, K., Kauf, P., Probst-Hensch, N., \& Brombach, C. (2017). Relative validation of a food frequency questionnaire to estimate food intake in an adult population. Food \& Nutrition Research, 61(1), 1305193. doi:10.1080/16546628.2017.1305193

Steinfeld, H., Gerber, P., Wassenaar, T., Castel, V., \& De Haan, C. (2006). Livestock's long shadow: environmental issues and options: Food \& Agriculture Org.

Stieger, S., Göritz, A. S., \& Burger, C. (2010). Personalizing the IAT and the SC-IAT: Impact of idiographic stimulus selection in the measurement of implicit anxiety. Personality and Individual Differences, 48(8), 940-944.

Stone, J., \& Fernandez, N. C. (2008). To Practice What We Preach: The Use of Hypocrisy and Cognitive Dissonance to Motivate Behavior Change. Social and Personality Psychology Compass, 2(2), 10241051. doi:10.1111/j.1751-9004.2008.00088.x

Strack, F., \& Deutsch, R. (2004). Reflective and impulsive determinants of social behavior. Personality and social psychology review, 8(3), 220-247. doi:10.1207/s15327957pspr0803_1

Sutton, S. (1998). Predicting and explaining intentions and behavior: How well are we doing? Journal of Applied Social Psychology, 28(15), 1317-1338.

Swanson, J. E., Swanson, E., \& Greenwald, A. G. (2001). Using the Implicit Association Test to investigate attitude-behaviour consistency for stigmatised behaviour. Cognition and Emotion, 15(2), 207-230. doi:10.1080/02699930125706

Teixeira, P. J., Carraca, E. V., Markland, D., Silva, M. N., \& Ryan, R. M. (2012). Exercise, physical activity, and self-determination theory: a systematic review. Int J Behav Nutr Phys Act, 9(1), 78. doi:10.1186/14795868-9-78

Teper, R., Segal, Z. V., \& Inzlicht, M. (2013). Inside the mindful mind: How mindfulness enhances emotion regulation through improvements in executive control. Current Directions in Psychological Science, 22(6), 449-454.

Terry, J. W., Erickson, C., \& Johnson, D. J. (1977). Changing habits by changing attitudes. Journal of Physical Education and Recreation, 48(7), 13-13.

Thrash, T. M., Elliot, A. J., \& Schultheiss, O. C. (2007). Methodological and Dispositional Predictors of Congruence Between Implicit and Explicit Need for Achievement. Personality and Social Psychology Bulletin, 33(7), 961-974. doi:10.1177/0146167207301018

U.S. Department of Health and Human Services. (2008). Physical activity guidelines advisory committee report. Retrieved from https://health.gov/paguidelines/report/

Van Assema, P., Brug, J., Ronda, G., Steenhuis, I., \& Oenema, A. (2002). A Short Dutch Questionnaire to Measure Fruit and Vegetable Intake: Relative Validity Among Adults and Adolescents. Nutrition and Health, 16(2), 85-106. doi:10.1177/026010600201600203

Van Buuren, S., Brand, J. P., Groothuis-Oudshoorn, C. G., \& Rubin, D. B. (2006). Fully conditional specification in multivariate imputation. Journal of statistical computation and simulation, 76(12), 1049-1064.

Vater, A., Schröder-Abé, M., Schütz, A., Lammers, C.-H., \& Roepke, S. (2010). Discrepancies between explicit and implicit self-esteem are linked to symptom severity in borderline personality disorder. Journal of behavior therapy and experimental psychiatry, 41(4), 357-364. 
Veenstra, E. M., \& de Jong, P. J. (2010). Restrained eaters show enhanced automatic approach tendencies towards food. Appetite, 55(1), 30-36. doi:https://doi.org/10.1016/j.appet.2010.03.007

Verbeke, W., \& Viaene, J. (1999). Beliefs, attitude and behaviour towards fresh meat consumption in Belgium: empirical evidence from a consumer survey. Food Quality and Preference, 10(6), 437-445.

von Hippel, W., Brener, L., \& von Hippel, C. (2008). Implicit Prejudice Toward Injecting Drug Users Predicts Intentions to Change Jobs Among Drug and Alcohol Nurses. Psychological Science, 19(1), 7-11. doi:10.1111/j.1467-9280.2008.02037.x

Vrabel, J., \& Zeigler-Hill, V. (2017). Conscious vs. Unconscious Determinants of Behavior. In V. Zeigler-Hill \& T. K. Shackelford (Eds.), Encyclopedia of Personality and Individual Differences (pp. 1-4). Cham: Springer International Publishing.

Walther, E., Ebert, I., \& Meinerling, K. (2011). Does Cue competition reduce conditioned liking of brands and products? Psychology \& Marketing, 28(5), 520-538.

Walthouwer, M. J. L., Oenema, A., Candel, M., Lechner, L., \& de Vries, H. (2015). Eating in moderation and the essential role of awareness. A Dutch longitudinal study identifying psychosocial predictors. Appetite, 87, 152-159.

Wantland, D. J., Portillo, C. J., Holzemer, W. L., Slaughter, R., \& McGhee, E. M. (2004). The effectiveness of Web-based vs. non-Web-based interventions: a meta-analysis of behavioral change outcomes. Journal of medical Internet research, 6(4), e40.

Warburton, D. E., Charlesworth, S., Ivey, A., Nettlefold, L., \& Bredin, S. S. (2010). A systematic review of the evidence for Canada's Physical Activity Guidelines for Adults. International Journal of Behavioral Nutrition and Physical Activity, 7(1), 39. doi:10.1186/1479-5868-7-39

Waters, A. J., Carter, B. L., Robinson, J. D., Wetter, D. W., Lam, C. Y., \& Cinciripini, P. M. (2007). Implicit attitudes to smoking are associated with craving and dependence. Drug \& Alcohol Dependence, 91(2), 178-186. doi:10.1016/j.drugalcdep.2007.05.024

Webb, T. L., Joseph, J., Yardley, L., \& Michie, S. (2010). Using the internet to promote health behavior change: a systematic review and meta-analysis of the impact of theoretical basis, use of behavior change techniques, and mode of delivery on efficacy. Journal of medical Internet research, 12(1), e4.

Webb, T. L., \& Sheeran, P. (2006). Does changing behavioral intentions engender behavior change? A meta-analysis of the experimental evidence. Psychological Bulletin, 132(2), 249.

Weinkauf, M. (2012). BenjaminiHochberg.xlsx $1.1 \mathrm{ed}$.

Weir, J. P. (2005). Quantifying test-retest reliability using the intraclass correlation coefficient and the SEM. The Journal of Strength \& Conditioning Research, 19(1), 231-240.

Wendel-Vos, G. C., Schuit, A. J., Saris, W. H., \& Kromhout, D. (2003). Reproducibility and relative validity of the short questionnaire to assess health-enhancing physical activity. Journal of Clinical Epidemiology, 56(12), 1163-1169. doi:10.1016/S0895-4356(03)00220-8

Westhoek, H., Lesschen, J. P., Rood, T., Wagner, S., De Marco, A., Murphy-Bokern, D., ... Oenema, O. (2014). Food choices, health and environment: effects of cutting Europe's meat and dairy intake. Global Environmental Change, 26, 196-205.

White, I. R., Royston, P., \& Wood, A. M. (2011). Multiple imputation using chained equations: issues and guidance for practice. Statistics in medicine, 30(4), 377-399.

WHO. (2003). Diet, nutrition and the prevention of chronic diseases - Introduction (0512-3054). Retrieved from $<$ Go to $|S|>: / /$ WOS:000184541900001

Wicker, A. W. (1969). Attitudes versus actions: The relationship of verbal and overt behavioral responses to attitude objects. Journal of Social issues, 25(4), 41-78.

Wiers, R. W., Eberl, C., Rinck, M., Becker, E. S., \& Lindenmeyer, J. (2011). Retraining Automatic Action Tendencies Changes Alcoholic Patients' Approach Bias for Alcohol and Improves Treatment Outcome. Psychological Science, 22(4), 490-497. doi:10.1177/0956797611400615

Wiers, R. W., Gladwin, T. E., Hofmann, W., Salemink, E., \& Ridderinkhof, K. R. (2013). Cognitive bias modification and cognitive control training in addiction and related psychopathology: Mechanisms, clinical perspectives, and ways forward. Clinical Psychological Science, 1(2), 192-212. 
Wiers, R. W., Van Woerden, N., Smulders, F. T., \& De Jong, P. J. (2002). Implicit and explicit alcohol-related cognitions in heavy and light drinkers. Journal of abnormal psychology, 111(4), 648.

Wilson, T. D., Lindsey, S., \& Schooler, T. Y. (2000). A model of dual attitudes. Psychological review, 107(1), 101.

World Health Organization. (2017). Physical activity. Fact sheet N 385: February.

World Health Organization [WHO]. (2010). Global recommendations on Physical Activity for health: World Health Organization.

Zeigler-Hill, V. (2006). Discrepancies between implicit and explicit self-esteem: Implications for narcissism and self-esteem instability. Journal of personality, 74(1), 119-144. 


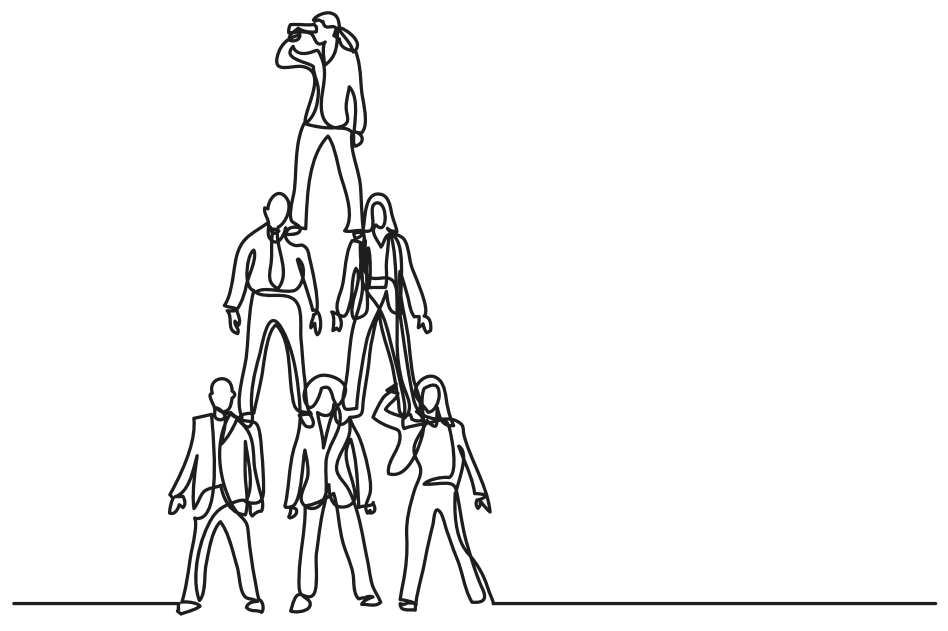




\section{Summary}

Valorization Addendum

Acknowledgements

About the author 


\section{Summary}

This dissertation aimed to gain a better understanding of how implicit attitudes and explicit cognitions relate to each other as well as how these different types of cognitions jointly predict health behaviors. To do so, we broadened the common approach of looking at implicit attitudes and explicit attitudes only in the prediction of (health) behaviors and added more explicit cognitions, which are known to be important determinants of behavior, into the analyses. The following research questions were addressed: (1) (How) do both types of cognitions jointly predict two different health behaviors? (2) What is the effect of dissonant implicit attitudes and explicit attitudes (IED) on the relationship between explicit attitude and intention/behavior? (3) Is mindfulness related to IED and can mindfulness buffer a possible negative effect of IED on intention/behavior? and (4) Do implicit attitudes, explicit attitudes, and behavior influence each other over time?

Regarding research question one, we found that implicit attitudes and explicit cognitions predicted two different health behaviors in accordance with the interactive pattern. More precisely, implicit attitudes moderated the relationship between self-efficacy and both behaviors. In both cases, the effect of self-efficacy on the respective behavior was strengthened when the implicit attitude pointed into the same direction (e.g. high self-efficacy to be physically active and a positive implicit attitude towards PA, high self-efficacy to reduce ones RMC and a negative implicit attitude towards RM). For the prediction of intention, support for the additive pattern (for RMC) as well as for the interactive pattern (for RMC and PA) was found, indicating that the two patterns do not exclude each other. Regarding the interactive pattern, the effect of the respective explicit cognitions on intention was again strengthened by those implicit attitudes that pointed into the same direction, e.g. the negative effect of perceived cons on PA intention was strengthened by negative implicit attitudes towards PA.

For the explicit cognitions concerning social influence (social norms, social modeling), a different pattern was detected. That is, when social norms or modeling of a behavior were strong, the intention to perform the behavior was strongest when implicit attitudes regarding the behavior/object were in the same direction (for RMC) or opposed (for PA and RMC) (e.g. perceiving many important others as sufficiently physically active and holding a negative implicit attitude towards PA or perceiving strong expectations of important others to reduce one's RMC and holding a positive implicit attitude towards red meat). This suggests that there can be dissonance between explicit constructs (social norms and social modeling) and a person's implicit attitude and furthermore, that individuals are motivated to resolve this dissonance. One way to do so could be by neglecting one's implicit attitude and by behaving in accordance with the more obvious norms and behaviors of important others. In the present studies such a dissonance reduction resulted in positive outcomes when considering from a health perspective (e.g. a high intention to be active as well as a high intention to reduce ones RMC). It is unclear, however, how this effect would look like, in case social influence was negative (e.g. no expectation of others to be physically active combined with a positive implicit 
attitude towards PA or low expectations to reduce RMC with a negative implicit attitude towards RM). Hence, more research is needed to assess under which specific circumstances dissonance between explicit cognitions and implicit attitudes can be beneficial or detrimental for the pursue of health behaviors. Although implicit attitudes were not found to have a direct influence on behavior, they did influence behavior indirectly. Based on this finding, it can be concluded that health interventions might profit from next to tackling explicit cognitions to also address implicit attitudes by steering them into the desirable direction. The latter approach could be implemented using evaluative conditioning.

Regarding the second research question, we did not find IED to moderate the relationship between the explicit attitude and intention and behavior. What we did find, however, was that IED was negatively associated with PA levels, even when the implicit attitude and the explicit attitude itself were not related to the behavior. The negative association between IED and PA indicates that IED can be detrimental for the pursue of health behaviors. This is presumably the consequence of an uncertainty about whether or not to perform the behavior, which in turn results from discrepant attitudes regarding the behavior. Hence, IED does not weaken the predictive power of explicit attitudes on intention and behavior but shows a direct association with behavior, which was negative in the current context. This suggests, that the congruence of attitudes should be taken into account when aiming at behavior change, even when implicit attitudes and explicit attitudes are not directly associated with the behavior.

In chapter 4, we addressed the third research question whether mindfulness is associated with IED and whether it can buffer a possible negative effect of IED on intention and behavior. Mindfulness subskills were neither related to IED nor did the mindfulness subskills moderate the relationship between IED and behavior. IED was, however, negatively associated with behavior and intention, in the sense that higher IED was associated with lower levels of RMC and a higher intention to reduce one's RMC. Again, it is likely that IED motivated people to dissolve the dissonance, which results from the different implicit and explicit attitudes towards the same behavior/object. The mindfulness subskill acceptance, however, was found to buffer, the aforementioned effect. That is, people who reported a high distinct ability to accept their inner feelings and processes as they are, did not indicate a higher intention to reduce their RMC (as an attempt to reduce dissonance) compared to people with a less distinct acceptance subskill. Hence, in addition to the known strategies to resolve dissonance, there seemingly exists an additional way to resolve dissonance and associated negative feelings, namely by the 'simple' acceptance of it. This finding appears especially valuable for research fields in which dissonant attitudes have been shown to be maladaptive, e.g. research regarding self-esteem. Future studies should investigate whether training the ability to accept can be used as an alternative to changing implicit attitudes, when dissonance is present.

Regarding the last research question whether implicit attitudes, explicit attitudes, and behavior influence each other over time, we did not find reciprocal relationships. Only the relationship between implicit attitude and perceived cons (regarding PA) was significant. The more positive the implicit attitude towards PA at baseline, the lower the perceived pros of 
PA at T1. This could be explained by means of the APE (Gawronski \& Bodenhausen, 2006). That is, the positive implicit evaluation of PA might not have been considered as valid, when forming one part of one's explicit attitude -perceived pros. This could be due to the fact that more perceived cons at baseline were also negatively related to perceived pros at T1. As perceived cons might be relevant propositions but are inconsistent with the implicit attitude, it is possible that the implicit attitude was considered as invalid when forming one part of one's explicit attitude i.e. perceived pros. In order to be able to draw more general conclusions about whether and how implicit attitudes, explicit attitudes, and behavior influence each other over time, we encourage future research in this avenue. 


\section{Valorization Addendum}

\section{TARGET GROUPS}

The influence of implicit cognitions is ubiquitous in our daily lives. Health behaviors, such as physical activity (Bluemke et al., 2010; Cheval et al., 2015; Conroy et al., 2010), smoking (McCarthy \& Thompsen, 2006; Perugini, 2005), alcohol consumption (McCarthy \& Thompsen, 2006), drug use (Rooke, Hine, \& Thorsteinsson, 2008; Wiers, Gladwin, Hofmann, Salemink, \& Ridderinkhof, 2013), or food choice (Deluchi, Costa, Friedman, Gonçalves, \& Bizarro, 2017; Richetin et al., 2007) have been shown to be linked to people's implicit cognitions. Also other behaviors, such as voting behavior (Arcuri et al., 2008; Friese, Smith, Plischke, Bluemke, \& Nosek, 2012), sustainability(-related) behavior (Panzone, Hilton, Sale, \& Cohen, 2016), or consumer preferences (Dimofte, 2010; Friese et al., 2006) are related to implicit cognitions. Also, mental health illnesses, such as anxiety or depression, are connected to implicit cognitions. More precisely, anxious or depressed individuals show a strong tendency to attend to threatening or negative stimuli, respectively, which in turn strengthen the illness (Bar-Haim, Lamy, Pergamin, Bakermans-Kranenburg, \& van ljzendoorn, 2007; Gotlib, Krasnoperova, Yue, \& Joormann, 2004; MacLeod et al., 1986). Furthermore, doctor-patient interactions (FitzGerald \& Hurst, 2017; Green et al., 2007; Hoffman, Trawalter, Axt, \& Oliver, 2016) or customer-client interactions are influenced by implicit cognitions. In July 2018, Starbucks Coffee closed more than 8.000 of its stores for a few hours in order to provide their staff with an implicit bias training concerning racial biases (Siegel, 2018). This was the consequence of an incident earlier that year, in which a store manager had called the police as two black men were sitting in the store without having purchased an order. White people, however, who were also sitting in the store without having purchased an order, did not suffer any consequences. This example demonstrates that implicit cognitions can be major influencers of our daily behaviors and Starbucks employees are surely not the only workers who demonstrate this. Further, it becomes clear that implicit cognitions have entered the mainstream and are no longer concepts discussed in the scientific context only.

So should all companies follow the example of Starbucks and offer an implicit bias training? It has to be made clear that changing implicit cognitions is not enough in order to change behavior. Although implicit cognitions are related to behavior, they are not the only determinants that influence behavior. Changing implicit cognitions can contribute to behavior change, yet they are not the only cognitions that should be addressed when aiming at behavior change as other explicit cognitions, such as explicit attitude, self-efficacy, or social norms are also important prerequisites for behavioral performance. Hence, the answer to the above-stated question should be "Yes, but this is not enough." As outlined in this dissertation, a change of implicit cognitions should ideally be combined with a change in explicit cognitions. Due to their omnipresence, the target groups concerning implicit and explicit cognition 
modification are broad. One could think of health promotion interventions that are aiming to stimulate people to perform more desirable health behaviors. However, not only interventions that are concerned with reversing unhealthy behaviors into healthier ones might profit from this approach, but also preventive interventions, for instance aimed at individuals who are at a higher risk to develop unhealthy behaviors (e.g. based on age, socio-economic status, gender, lifestyle, or environmental factors). The same applies to the mental health sector. Not only individuals who already suffer from mental diseases might profit from an implicit and explicit cognition modification, but also subclinical individuals who show a strong attentional bias towards threatening or negative stimuli. It is likely that latter ones are at increased risk of making a transition from a non-clinical to a clinical state. In order to prevent this, early measures, such as implicit and explicit cognition modification, might be helpful. This might be especially attractive for health care insurances in order to prevent unhealthy behaviors to develop further and to save health care costs. Furthermore, in order to prevent or decrease racial behaviors (concerning gender, ethical background, or skin colour) organizations could also make use of implicit cognition trainings in addition to explicit measures.

\section{N NOVATION}

Previous research concerning the integration of implicit and explicit cognitions in the prediction of behavior focused mainly on the interplay between implicit attitudes and explicit attitudes. The approach of combining implicit attitudes with more explicit cognitions (besides explicit attitudes) in the prediction of behavior has not been followed extensively before. Moreover, intention has not been included in theoretical assumptions, although it forms one of the most important prerequisites for behavioral performance. The novel and innovative approach of combining implicit attitudes with more explicit cognitions in the prediction of behavior allowed us to better understand how the two different types of cognitions relate to each other as well as how they jointly determine health behaviors. Thereby the contemporary notion that behavior is a combined result of conscious and rather unconscious mechanisms was supported. Although implicit attitudes were not directly related to behavior, they were directly related to intention. Furthermore, they influenced the well-known relations between explicit cognitions and intention and behavior. This knowledge was undiscovered until now. Hence, both types of cognitions should be changed in order to achieve more effective outcomes concerning behavior change. As this approach is normally not followed in current interventions, future interventions should be informed by these findings and should apply this strategy to achieve even more successful outcomes. This might not only contribute to improved health and greater well-being in people but also to reduced health care costs. 


\section{ACTIVITIES AND PRODUCTS}

The computer and the Internet have become a prominent device and channel to deliver interventions in a far-reaching as well as a cost-effective way (Wantland, Portillo, Holzemer, Slaughter, \& McGhee, 2004). One intervention method that is used frequently is tailoring, meaning that depending on the individual's pre-existing cognitions, the individual receives tailored advice or tasks he or she has to perform to result in a change of specific cognitions. These types of interventions could be nicely complemented by evaluative conditioning tasks to steer not only explicit cognitions but also implicit attitudes into the 'right' direction. Also, the constantly growing dissemination and usage of smartphones in Europe and worldwide, make smartphones a promising channel to bridge the gap between the accessibility of interventions and their mobility. Apps based on gamification could offer an interactive, playful, and attractive way to deliver evaluative conditioning tasks and methods to change explicit cognitions. For example, Dennis and O'Toole (2014) developed a mobile game application based on the dot-probe task (MacLeod et al., 1986) in order to retrain attentional biases in highly anxious adolescents. More precisely, participants were shown two cartoon characters on the screen, which were presented in grassland. One character showed a neutral/positive facial expression whereas the other one showed an angry expression. Simultaneously, both characters burrowed themselves in the grass field and a trail of grass appeared only there, where the character with the neutral/positive expression was burrowed. Participants were asked to indicate this trail of grass as quickly as possible and it was demonstrated that the focus on positive/neutral faces resulted indeed in a reduced attentional bias. Although this app addressed attentional bias and not implicit attitudes, it is imaginable that similar applications could be used in order to change implicit attitudes. By this means, a broad target group could be reached.

Another approach to combine implicit and explicit cognition change is entertainmenteducation and serious gaming, which are both understood as an intersection between entertainment and education. Serious games can aim at behavior change and can at the same time be experienced as enjoyable and intrinsically motivating as they use certain techniques, such as the flow effect, reinforcement, goal-setting, positive feedback, or social connectivity (DeSmet, Shegog, Van Ryckeghem, Crombez, \& De Bourdeaudhuij, 2015; Graesser, Chipman, Leeming, \& Biedenbach, 2009). Thereby, rather repetitive tasks to change implicit attitudes could be delivered in an appealing and fun way. One example is the serious game 'Heroes of the night', in which implicit attitudes regarding protective sexual behaviors were addressed by means of different tasks, amongst others evaluative conditioning (Brüll, Hebecker, Wiers, Kok, \& Ruiter, under review). More precisely, the avatar received positive or negative audio and visual feedback after having engaged in (un-)safe sexual behavior. Hence, the desired behavior was paired with something positive whereas the unwanted behavior was paired with something negative. 
Taken together, serious games or apps based on gamification offer a richer and more interesting context to deliver implicit modification tasks, which might be perceived as rather repetitive and boring. Although the abovementioned applications are based on the original paradigms, there still appears to be a risk to lose some of the key elements of the original paradigms, when embedding them in a serious game (Boendermaker, Peeters, Prins, \& Wiers, 2017). Thereby it is possible that the intervention/paradigm becomes less effective than the pure original paradigm itself. In order to rule out this risk, the effectiveness of these games should be compared to the effectiveness of the original paradigms before using them as a promising alternative. Another possibility could be to add game elements around the original paradigm, thereby leaving the task unchanged but still motivating the user by rewarding good task performance after his or her performance. Complementing these types of games with measures to change explicit cognitions offer a promising avenue for prospective interventions. It is now the task for future research to investigate the best method to create and deliver interventions that are able to change both explicit and implicit cognitions. 


\section{Acknowledgements}

Dear Hein, I was very happy when you called me around 5 years ago to tell me that you had selected me for the PhD project. I know that it was a very tight decision between me and another applicant and, therefore, I was and still am especially thankful for the trust you put in me. I also thank you for stimulating me to think outside of the box, by playing the advocate of the devil. Although it was not always easy for me to deal with the advocate's devil, it surely made me grow in my scientific thinking, my ability to reflect critically as well as in my ability to convince other people of my ideas. Thank you for letting me grow in these abilities and skills! Throughout the years, I also appreciated your encouragement to present my work at national and international conferences, which did not only help me to create my own network but also made me grow on a personal level. Thank you for these possibilities!

Dear Rik, you joined my team when I was already/only halfway through and that was a true blessing for me. Thank you for being a mentor that I look up to and feel inspired by. It's not only that you gave me so much autonomy and freedom to follow my own way of working, but also a lot of trust going along with that. You offered me support, intellectual food, and your door was always open for me, which I appreciated so much. I always enjoyed our brainstorms and after each meeting with you, I was able to continue with my project, no matter how stuck I thought I had been. In your presence, I knew that there were no stupid questions to ask but only good answers to find - together and on the same level. Thank you from the bottom of my heart for always seeing the person in me.

Dear Iman, thank you very much for taking me by the hand when I took my first baby steps into the big unknown world, called 'Science'. I appreciated your calm nature, which you even maintained in the midst of a storm. This quality helped me a lot to also stay calm and gave me lots of confidence. Thank you for accompanying me on my way to become a grown-up scientist.

Dear Math, thank you very much for your help regarding statistical questions and analyses, for taking the time to explain complex matters in a very easy, understandable and precise way, and for the valuable feedback you gave on my manuscripts.

Stan aka Stani, Stanislav, StanislaSSS, Stommislav, Stommi. Thank you for being and staying a musketeer throughout the past years. It was a blessing for me that your office was right at the opposite of mine and that I could always even binnenstormen for a quick or very long chat about our (PhD) lives. You are like a brother to me, who gave me a lot of stability, joy, and motivation throughout the years. I especially thank you for making me laugh at the same jokes over and over and over and over and over and over and over again. I will never forget the good laughs we shared, the serious conversations, the boterhammen you fed me with, when I 
was running out of food, the great conference experiences/dinners, the musical intermezzos, the superFIT balls which you enjoyed throwing into my face so much as well as the help and support we offered to each other. Thank you, bro!...5,6,7,8! One for all, all for one!

Adriana. Wow, what an incredible little strong woman you are. We only got to know each other in 2018 and what came out of the relatively short time we shared at our department is a beautiful friendship, which I appreciate a lot. You are mentally so strong, always optimistic, so caring and so smart. You inspired me so often with your optimism, positive energy, your understanding and your positive mindset that it was a true blessing for me to meet you in the final phase of $\mathrm{mf}$ PhD. You encouraged me in difficult times and always said 'Het komt goed', and you were always right. Thank you for your friendship and your support! I hope that this beautiful friendship will last for a very long time!

Niki. Another little, yet very strong women. Dear Niki, thank you for your genuine interest in me as a person and in my life. I always loved our deep and funny conversations and was always surprised by your attentiveness. To me, you are not only one of the most attentive women I know, but also one of the bravest. Not only, because you completed the Iron Man but much more because you stayed true and honest to yourself when deciding to stop with your project. This requires a lot of courage and strength and was a true inspiration for me. Thank you for your friendship inside and outside of the work environment and the very special dance move we share with each other...5,6,7,8!

Lieber Tommi, danke, dass ich dein Ruhrpöttchen sein durfte/darf und jeden "Morgen" deinen Namen laut über den Flur rufen durfte. Du bist für jeden Scherz zu haben und noch viel wichtiger, hast mir in vielen Momenten eine Schulter zum Anlehnen gegeben. Vielen Dank dafür! Danke auch, dass ich in dir einen Verbündeten gefunden habe, mit dem ich die Begeisterung und den Enthusiasmus für Mindfulness und einige andere spirituelle Themen teilen kann - wer hätte das gedacht, als wir uns vor ein paar Jahren kennengelernt haben (:)

Dirk-Jan aka Mourik or Mourislav. Thank you very much for your companionship throughout the last years! I was always happy to come to your office and to be the 'koffie-politie' - although you stopped drinking coffee at some point. I am not sure how you managed to survive your PhD without coffee, but I guess you compensated with lots of avocados. Thank you for the talks and laughs we shared with each other and thank you for throwing me out of your office from time to time, reminding me that there was a project awaiting to be finished.

Dear Ilona! What a pleasure it was for me to share the office with such a calm and relaxed person! It was great to share so many laughs with you but also so many serious and honest conversations. I always appreciated your openness, the space you gave me for me and my project, your understanding and your trustworthy nature. Thank you for singing Coldplay 
songs along with me and for creating such a lovely and relaxed environment, which I enjoyed working in a lot. I wish you all the best for finishing your own project.

Dear Anke, to me you are the good soul of the department! Always full of energy and constantly spreading positive vibes! Thank you for offering me a shelter, while my food was heating up in the microwave, your styling advises, your travel tips, your good taste in coffee, which gave me a companion for going the 'long' way to the good coffee places, and for affecting me with your good moods. Please always stay as positive as you are!

Stroganov! What can I say about you? Well, when I didn't know you that well, I thought you were arrogant. When I got to know you better, I found you unbelievably funny and honest. Thank you for your open ear in difficult times and your great ability to emphasize with other people! Thank you for the many endless critical discussions we had about mindfulness and positive psychology, which I could only have with you, as you are one of the most critical people I know. So thank you for these and many other interesting and gezellige talks, coffees, appelflapjes, and for eating coconut yoghurt for me, although you hate coconut like hell!

Dear Kei-Long. You definitely were the most interesting person I shared my office with and I can say that I got a lot of inspiration from you! You reminded me that every person carries his or her own package and that we should not judge people because they are different. Thank you for these reminders! I admire your authenticity and your confidence to be yourself, no matter what other people think. I think this is a very valuable quality of yours!

Dear Gerjanne. Thank you for being my kamergenoot in the final months of my project. We actually didn't see each other very often, but when we happened to be in the office at the same time, you were always so lovely and adorable and our talks always gave me a lovely break from my work. Thank you for respecting me, my work, and the way I was. I wish you good luck with finishing your own PhD!

Dear Leon, you were and will probably be the only person in my life, who called me 'vieh'. Thank you for this special title! I will surely miss being addressed in this way! Most of all, I thank you for your technical support, when I was encountering problems with Endnote, Word, Excel, Spss, Power Point, or some strange reaction time tasks, which no one of the department had ever used before. It was a true blessing for me that there was always someone (although it felt as if you were in Spain every second week), who knew how to shut down and restart my computer. This great expertise of yours saved my work so often! ;-)

Liebe Familie, vielen Dank für eure bedingungslose Unterstützung und eure Liebe - heute sowie früher. Ich bin besonders dankbar dafür, dass du Mama, dich immer dafür eingesetzt hast, dass wir eine gute Bildung genießen. Ohne deine Willenskraft wäre es sicher nie so weit gekommen, dass ich mal promovieren würde. Ich danke dir von Herzen dafür! 
Liebe Familie Figge, vielen Dank dafür, dass ihr mich von Anfang an so herzlich und bedingungslos in eure Familie aufgenommen habt - wie eine Schwester oder Tochter. Vielen Dank für eure Unterstützung und die schönen Momente, die wir bisher zusammen verbringen durften, von denen hoffentlich noch unendlich weitere folgen werden.

Sarah. Mein lieber Sohnemann! Wer hätte gedacht, dass eine Begegnung an einer Säule von solcher Bedeutung sein könnte! Ich bin so froh, dass ich dich damals in Maastricht kennengerlernt habe. Du hast meine Zeit dort zu einer ganz besonderen Zeit für mich gemacht. So besonders, dass ich gar nicht mehr aus Maastricht weg wollte. Auch wenn wir jetzt nicht mehr in derselben Stadt wohnen, nährt unsere Freundschaft mich immer noch sehr, bist du doch die einzige, mit der ich einen ganz speziellen Humor teilen kann. Vielen Dank für deine Freundschaft, deinen Humor, deine Großzügigkeit und deine Einzigartigkeit! Ich freue mich schon auf unsere gemeinsame Zeit im Altenheim!

Christina und Katrin. Meine lieben weisen Freundinnen! Wie schön, dass es euch gibt und dass wir uns kennengelernt haben. Ich bin so unglaublich dankbar für eure weisen Worte und Ratschläge und eure Reflektionsanstösse, die mir in vielen Situationen weitergeholfen haben. Danke für eure Urteilsfreiheit, eure emotionale Intelligenz und euer Einfühlungsvermögen. Ihr versteht mich so gut, wie nur wenige Menschen auf dieser Welt. Danke für eure Freundschaften, die mir so viel Inspiration, Hoffnung und Zuversicht geben! Ich hoffe sehr, dass diese noch bis in die Ewigkeit anhalten werden!

Anais. Mi chica favorita! I am so happy that I met you a few years ago, when I was attending one of your wonderful yoga classes. It's not only that you made me fall in love with yoga, but also with your beautiful nature! Thank you for your yoga classes, which helped me to calm down my sometimes busy mind, to reconnect to myself, and to relaxxxxxx, relaxxxxxx! I learned so much from you especially that one's mind can be one's greatest enemy but also one's best friend and that wisdom doesn't come with age but with an open mind and an open heart! You were a true inspiration for me when I saw you playing the piano so beautifully at your last exam, showing me that anything can be achieved with dedication and persistence, no matter how many obstacles one encounters. Thank you for that!

I also would like to thank the TMO social club for making working at GVO zo gezellig; Steffi for your contribution to a great start into the $\mathrm{PhD}$ life; Latifa for your extremely helpful nature regarding anything; Kim and Patricia for helping with all administrative matters around (finishing) the PhD; all my lovely students, especially Rosa and Marie, for being so eager and enthusiastic about learning and my way of teaching; Phil Brüll, for helping me to prepare for the job interview around 5 years ago and for many good advises around the PhD life throughout the years; Hugo Alberts for introducing me to mindfulness, for making me fall in love with this concept, and for supervising me on this topic during my research internship; 
Axelle de Rore for your beautiful and energizing dance classes and all the lovely ladies, who make the classes so enjoyable; all persons I met at Plum Village, especially Christine and Efrat, who showed me that there are very beautiful people out there in the world; the many pilgrims I met on the Camino(s) de Santiago, especially Katharina, Lars, and Pierre, for inspiring me in any imaginable way and for nurturing my soul; and the guidance I received, which I've never seen but surely met and of which I know that it was always there.

Lieber Lukas, was hätte ich in den letzten Jahren nur ohne dich gemacht... Du warst immer an meiner Seite, egal was war. Du hattest immer einen guten Ratschlag für mich parat, wenn ich mal nicht weiter wusste oder zwei starke Arme, die mich immer wieder auffingen. Ebenso warst du immer da, um die schönen Dinge im Leben mit mir zu feiern! Dafür danke ich dir von Herzen! Danke, dass du nicht nur mein Mann bist, sondern auch mein bester Freund, mit dem ich die Liebe für das Leben, die Natur, das Reisen, für Mindfulness und die Spiritualität teilen kann - Dinge, die mir so oft neue Energie geben und gegeben haben. Danke, dass du mich so nimmst, wie ich bin, dass wir gemeinsam immer wieder neue Abenteuer erleben, dass ich immer auf dich zählen kann, dass du nie von meiner Seite weichst und dass wir gemeinsam unsere Träume verwirklichen! Honey you are a rock, upon which I stand... 


\section{About the author}

Carolin Muschalik was born on the $1^{\text {st }}$ of May in Essen, Germany. After completing secondary school at the Leibniz Gymnasium in Essen, she started studying Cognitive and Media Science at the University Duisburg-Essen, in 2007. After having obtained her bachelor's degree, she obtained a master's degree in the same subject with an emphasis on social psychology. During that program, Carolin spent one semester abroad at Newcastle University, England, and noticed that she enjoyed studying abroad and in an international context. Also, she realized that she is very passionate about psychology and the understanding of human behavior and that she would like to learn even more about it, preferably abroad. Therefore, she started the Health and Social Psychology Master at Maastricht University in 2013 and graduated from this program one year later 'cum laude'. During her research internship on mindfulness and personal strengths use, supervised by Dr. Hugo Alberts, she realized that she is passionate about research and would like to learn more about it. Therefore, she started working as research assistant for various projects at the psychology department of Maastricht University. During this time, Carolin further developed her interest in research as she wanted to get even more insight into the whole process of conducting research.

In March 2015, Carolin started working as a PhD candidate at the department of Health Promotion at Maastricht University and was supervised by Prof. dr. Hein de Vries, Prof. dr. Rik Crutzen and dr. Iman Elfeddali. In the following four and a half years, she worked at several studies that are presented in this dissertation. She presented her work at several national and international scientific conferences and was awarded the price for an outstanding poster presentation at the European Public Health Conference in Padua, Italy, in 2017. In addition to her research, she was involved in various teaching responsibilities for bachelor and master courses in Health Promotion and Psychology. Also she was part of Green Impact, a change and engagement program that aims to bring positive social, environmental, and economic changes at universities.

In the future, Carolin would like to pursue a work, which allows her to combine the skills she obtained throughout her PhD with her personal interests for mindfulness, yoga, nature, and sustainability. 


\section{PUBLICATIONS PRESENTED IN THIS DISSERTATION}

Muschalik, C., Elfeddali, I., Candel, M. J. J. M., \& de Vries, H. (2018). A longitudinal study on how implicit attitudes and explicit cognitions synergistically influence physical activity intention and behavior. BMC Psychology, 6(1), 18. doi:10.1186/s40359-018-0229-0

Muschalik, C., Elfeddali, I., Candel, M. J. J. M., Crutzen, R., \& de Vries, H. (2019). Does the discrepancy between implicit and explicit attitudes moderate the relationships between explicit attitude and (intention to) being physically active? BMC Psychology, 7(1), 52. doi:10.1186/s40359-019-0322-z

Muschalik, C., Crutzen, R., Candel, M. J. J. M., Elfeddali, I. \& de Vries, H. (under review). Implicit Attitudes and Explicit Cognitions Jointly Predict Reduced Red Meat Intake: a Three-Wave Longitudinal Study

Muschalik, C., Crutzen, R., Elfeddali, I. \& de Vries, H. (in press). Mindfulness is not Associated with Dissonant Attitudes but Enhances the Ability to Cope with them.

Muschalik, C., Candel, M. J. J. M., Crutzen, R., Elfeddali, I. \& de Vries, H. (under review). Pathways of Implicit Attitudes, Explicit Attitudes, and Behavior. 
"And into the forest I go, to lose my mind and find my soul" John Muir 
